A I I I O

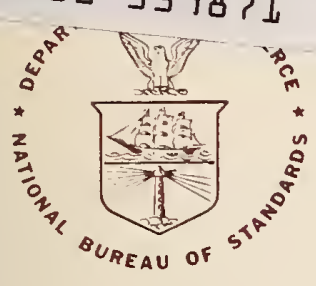

WEDEWG:

NBS

PUBLICATIONS

U.S. DEPARTMENT OF COMMERCE / National Bureau of Standards

\title{
Design, Evaluation, and Use of a Reverberation Chamber for Performing Electromagnetic Susceptibility/Vulnerability Measurements
}

M. L. Crawford

G. H. Koepke 
he National Bureau of Standards' was established by an act of Congress on March 3, 1901. The

Bureau's overall goal is to strengthen and advance the nation's science and technology and facilitate their effective application for public benefit. To this end, the Bureau conducts research and provides: (1) a basis for the nation's physical measurement system, (2) scientific and technological services for industry and government, (3) a technical basis for equity in trade, and (4) technical services to promote public safety. The Bureau's technical work is performed by the National Measurement Laboratory, the National Engineering Laboratory, the Institute for Computer Sciences and Technology, and the Institute for Materials Science and Engineering.

\section{The National Measurement Laboratory}

Provides the national system of physical and chemical measurement; coordinates the system with measurement systems of other nations and furnishes essential services leading to accurate and uniform physical and chemical measurement throughout the Nation's scientific community, industry, and commerce; provides advisory and research services to other Government agencies; conducts physical and chemical research; develops, produces, and distributes Standard Reference Materials; and provides calibration services. The Laboratory consists of the following centers:
- Basic Standards ${ }^{2}$

- Radiation Research

- Chemical Physics

- Analytical Chemistry

\section{The National Engineering Laboratory}

Provides technology and technical services to the public and private sectors to address national needs and to solve national problems; conducts research in engineering and applied science in support of these efforts; builds and maintains competence in the necessary disciplines required to carry out this research and technical service; develops engineering data and measurement capabilities; provides engineering measurement traceability services; develops test methods and proposes engineering standards and code changes; develops and proposes new engineering practices; and develops and improves mechanisms to transfer results of its research to the ultimate user. The Laboratory consists of the following centers:
- Applied Mathematics

- Electronics and Electrical Engineering ${ }^{2}$

- Manufacturing Engineering

- Building Technology

- Fire Research

- Chemical Engineering?

\section{The Institute for Computer Sciences and Technology}

Conducts research and provides scientific and technical services to aid Federal agencies in the selection, acquisition, application, and use of computer technology to improve effectiveness and economy in Government operations in accordance with Public Law 89-306 (40 U.S.C. 759), relevant Executive Orders, and other directives; carries out this mission by managing the Federal Information Processing Standards Program, developing Federal ADP standards guidelines, and managing Federal participation in ADP voluntary standardization activities; provides scientific and technological advisory services and assistance to Federal agencies; and provides the technical foundation for computer-related policies of the Federal Government. The Institute consists of the following centers:
- Programming Science and Technology

- Computer Systems Engineering

\section{The Institute for Materials Science and Engineering}

Conducts research and provides measurements, data, standards, reference materials, quantitative understanding and other technical information fundamental to the processing, structure, properties and performance of materials; addresses the scientific basis for new advanced materials technologies; plans research around cross-country scientific themes such as nondestructive evaluation and phase diagram development; oversees Bureau-wide technical programs in nuclear reactor radiation research and nondestructive evaluation; and broadly disseminates generic technical information resulting from its programs. The Institute consists of the following Divisions:
- Inorganic Materials

- Fracture and Deformation ${ }^{3}$

- Polymers

- Metallurgy

- Reactor Radiation 


\title{
Design, Evaluation, and Use of a Reverberation Chamber for Performing Electromagnetic Susceptibility/ Vulnerability Measurements
}

\author{
M. L. Crawford \\ G. H. Koepke
}

Electromagnetic Fields Division

Center for Electronics and Electrical Engineering

National Bureau of Standards

Boulder, Colorado 80303

\section{Supported in part by}

Naval Surface Weapons Centers (NSWC)

Dalgren, Virginia

$$
\text { and }
$$

Rome Air Development Center (RADC)

Griffiss Air Force Base

Rome, New York

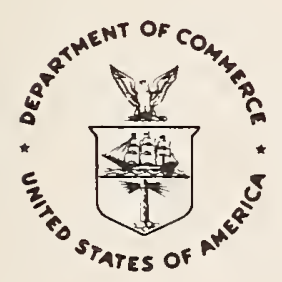

U.S. DEPARTMENT OF COMMERCE, Malcolm Baldrige, Secretary

NATIONAL BUREAU OF STANDARDS, Ernest Ambler, Director 
National Bureau of Standards Technical Note 1092

Natl. Bur. Stand. (U.S.), Tech Note 1092, 146 pages (Apr. 1986)

CODEN:NBTNAE 


\section{FOREWORD}

The research described in this report represents the culmination of over 3 years of work at the National Bureau of Standards (NBS), Electromagnetic Fields Division, Boulder, Colorado, to carefully evaluate, develop (when necessary), describe, and document the methodology for performing radiated susceptibility/vulnerability (EMS/V) measurements using a reverberation chamber. This effort was jointly funded by the Naval Surface Weapons Center (NSwC), Dahlgren, Virginia; Rome Air Development Center (RADC), Griffiss Air Force Base, Rome, New York; and the NBS. Three major tasks were outlined. These were to:

1. Determine how well a shielded enclosure could be made to operate as a reverberation chamber to establish time averaged (statistically determined), randomly polarized, uniform EM fields as a function of spatial position, frequency and chamber quality factor (Q);

2. Evaluate interaction effects between the chamber and equipment under test (EUT) placed inside the chamber to determine:

a. The range of applications of the measurement techniques (i.e., where the technique could or should not be used),

b. Whether measurement results obtained using the reverberation chamber could be correlated with results obtained using anechoic chambers or open field sites, and

c. To identify pertinent chamber calibration parameters;

3. Develop a technique applications and use manual.

These tasks were addressed sequentially in three major phases. The first phase was to design, construct, assemble and evaluate an empty (without the presence of an EUT inside) reverberation chamber with associated instrumentation and software to evaluate its electrical performance characteristics. This phase of the work began in January 1982 and was completed by the end of 1983. Results of this effort are contained in section 2 of this report. Section 2 discusses the modification of a $2.74 \mathrm{~m} \times 3.05 \mathrm{~m} \times 4.57 \mathrm{~m}$ welded steel shielded enclosure located at NBS into a reverberation chamber. Information given includes theoretical concepts, physical design and construction details, and the evaluation of the empty chamber's electrical parameters. These parameters are excitation and reference receiving antennas voltage standing wave ratio (VSWR), loss, Q factor, wave impedance, spatial E-field distribution versus frequency, and determination of the test field level.

The second phase of the work was to evaluate the interaction effects between the chamber and the EUT placed in the chamber. This effort occupied the latter part of 1983 through 1984. The results are contained in section 3 of this report. The third phase was to develop methodology and evaluate the use of the reverberation chamber for susceptibility measurements and compare the results to other more conventional measurements techniques such as anechoic chambers and to other reverberation chambers. This study included the selection and susceptibility evaluation of four samples of EUTs: a one centimeter dipole probe, a ridged horn antenna, a series of rectangular TEM transmission cells with apertures and a modified 7.0 cm (2.75") diameter folded fin aircraft rocket (FFAR). The first three "reference standard" EUT's were selected because they could be characterized theoretically. The fourth EUT (FFAR) was more typical of an operational EUT which is more indicative of the actual type of equipment routinely tested for EM susceptibility. Results were obtained for this phase of the effort during 1984 and 1985. Details of how the measurements were performed, including setups, approaches, and instrumentation and software requirements are contained in section 4. The final task was to document these results, presented in section 5 , and to produce a reverberation measurement technique applications and use manual. This, of course, is one of the purposes of this report. Section 6 gives a brief summary and conclusions drawn from this study. Some of the more significant are: 1) the NBS chamber lower useful frequency limit is $200 \mathrm{MHz}$; 2) the spatial variation in the statistically determined E-field established in the chamber is approximately $\pm 8 \mathrm{~dB}$ at $200 \mathrm{MHz}$ and decreases to $\pm 2 \mathrm{~dB}$ at $2 \mathrm{GHz}$; 3) the preferred measurement approaches are mode tuned (explained in section 4.3.2) at frequencies below 2 GHz and mode stirred (also explained in section 4.3.2) at frequencies above 2 GHz; 4 ) the 10 ading effect, of lowering the chamber's $Q$ by inserting the EUT, should be limited to an increase in chamber loss $\leqq 6 \mathrm{~dB}$ or a minimum tuner effectiveness $\geqq 20 \mathrm{~dB} ; 5$ ) the average wave impedance in the chamber is approximately $120 \pi$; 6) the maximum E-field is approximately 7-8 dB greater than the average E-field established in the chamber; 7) scattering from EUT does not significantly influence the E-field statistical, spatial distribution; 8) the directional characteristics of antenna or EUT placed inside the chamber are effectively lost; 9) the response of EUT measured inside a reverberation chamber is less than when measured inside an anechoic chamber (open space) in proportion to the EUT open space gain; 10) the response of EUT to an interference 
field after it has penetrated the EUT's shield appears to be equivalent in both reverberation and anechoic chambers; and 11) the experimental error analysis suggests that Root Square Sum (RSS) errors in establishing the test field inside a reverberation chamber range from approximately $\pm 10 \mathrm{~dB}$ at $200 \mathrm{MHz}$ to $\pm 4 \mathrm{~dB}$ at $18 \mathrm{GHz}$.

Section 7 provides suggestions for future research and Section 8 acknowledges the significant contribution of others to this project. References cited are given in Section 9. 
Abstract. .1

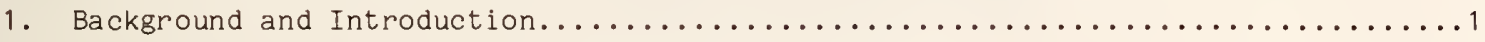

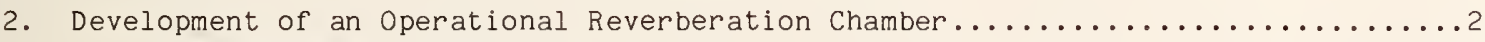

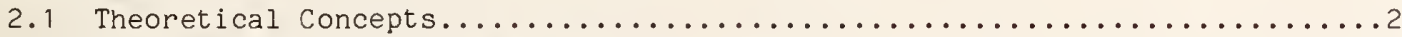

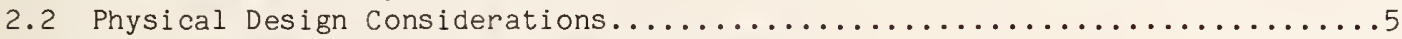

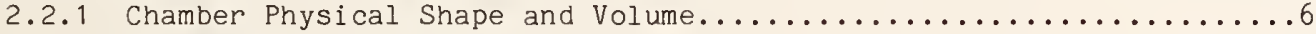

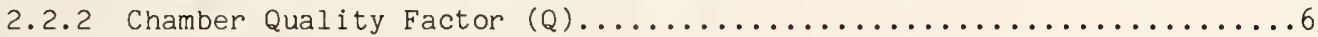

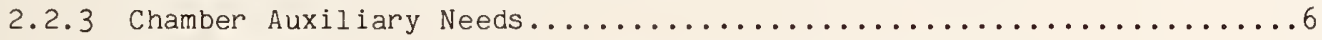

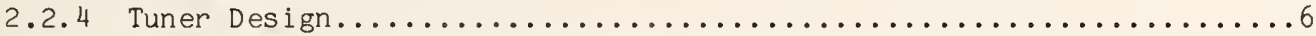

2.2.5 Chamber Excitation and Field Monitoring...................

2.3 Determination of Wave Impedance and E-Field Amplitude Distribution

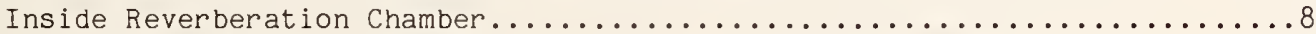

2.3.1 Determining the Test Field and Wave Impedance Amplitudes.........8

2.3.2 Maximum Versus Average Amplitude Responses..................9

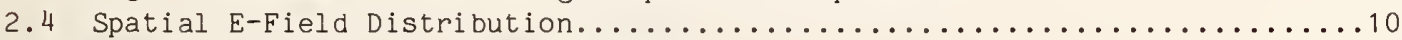

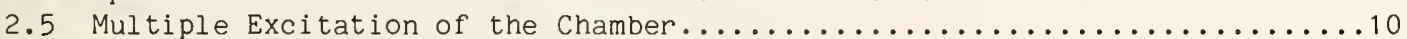

3. Interaction Effects Between Chamber, Source and Reference Antennas, and

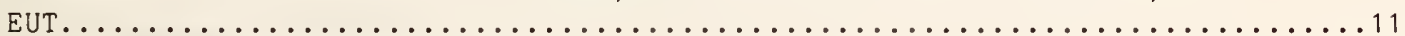

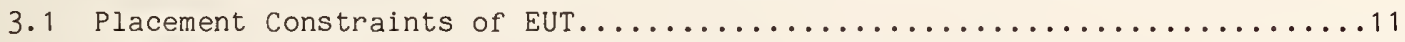

3.2 Scattering Effects of Metal Objects Upon E-Field Distribution..........11

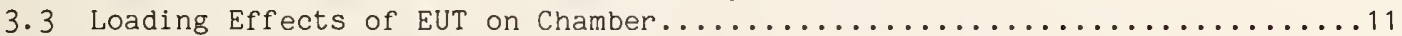

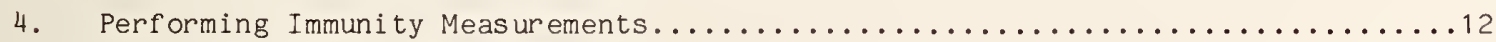

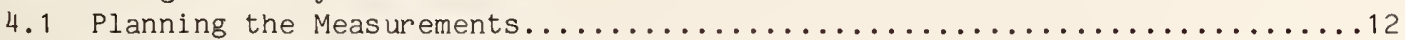

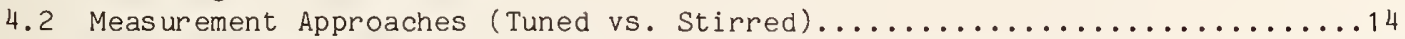

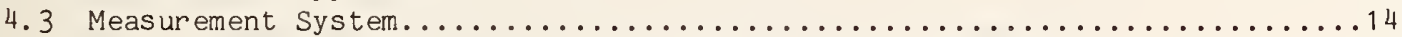

4.3.1 Measurement Setup and Instrumentation Requirements............ 14

4.3.2 Software Requirements and Measurement Procedures..............14

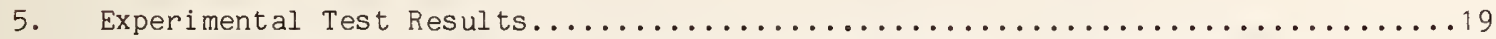

5.1 Correlation of Results to Anechoic Chamber Tests - Some Examples........19

5.1 .1 Description of Anechoic Chamber Measurement System..............19

5.1.2 Measurement of the Peak Response of a $1 \mathrm{~cm}$ Dipole and a

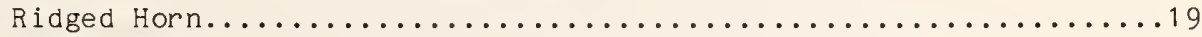

5.1 .3 Measurement of RF Coupling Through Apertures in TEM Lines.......20

5.1 .4 Measurements of the EM Susceptibility of a Modified $7.0 \mathrm{~cm}$

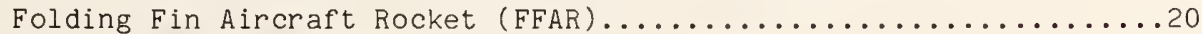

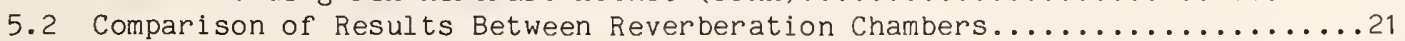

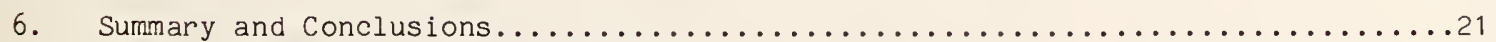

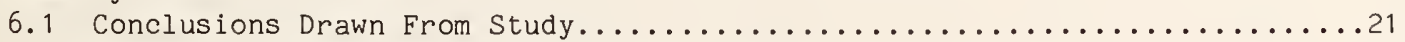

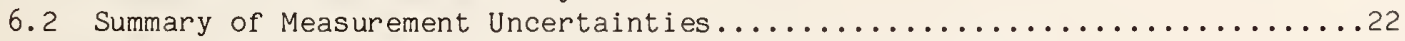

6.2 .1 Determining the Field Strength Inside the Chamber..............22

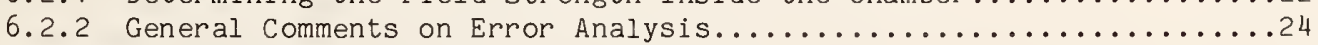

6.3 Measurement Technique Advantages and Limitations...................... 4

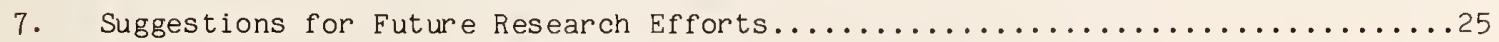

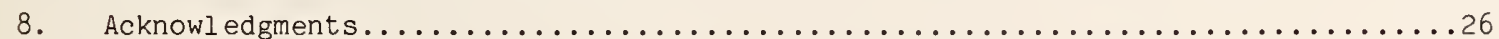

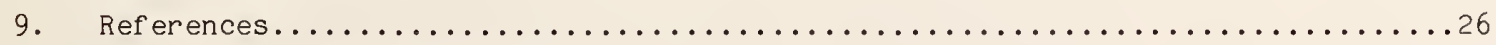

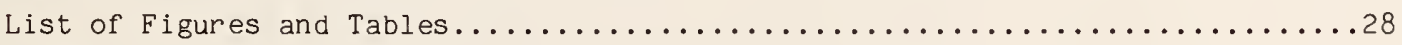

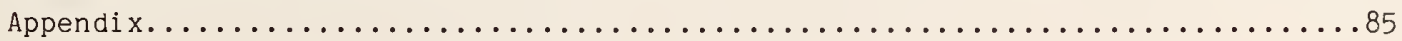



Design, Evaluation, and Use of a Reverberation Chamber for Performing Electromagnetic Susceptibility/Vulnerability Measurements

\author{
M.L. Crawford and G.H. Koepke \\ Electromagnetic Fields Division \\ National Bureau of Standards \\ Boulder, Colorado 80303
}

\begin{abstract}
This report presents the results of work at the National Bureau of Standards, Boulder, Colorado, to carefully evaluate, document, develop (when necessary), and describe the methodology for performing radiated susceptibility/vulnerability measurements using a reverberation chamber. The report describes the reverberation chamber theory of operation, construction, evaluation, functional operation, and use for performing immunity measurements. It includes an estimate of measurement uncertainties derived empirically from test results and from comparisons with anechoic chamber measurements. Finally, it discusses the limitations and advantages of the measurement technique to assist potential users in determining the applicability for this technique to their electromagnetic compatibility (EMC) measurement needs.
\end{abstract}

Key Words: electromagnetic susceptibility/vulnerability; estimated uncertainty; evaluation; measurement procedures; reverberation chamber

\title{
1. Background and Introduction
}

The use of mode tuned or stirred reverberation chambers for performing EMC measurements is a relatively new method which appears to have considerable potential for electromagnetic susceptibility/vulnerability testing [1-7]. The idea of reverberating a shielded enclosure as a way to improve the EMC measurement results obtained using the enclosure was first proposed in 1968 [1]. Since then measurement procedures have been developed for implementing the technique for both radiated susceptibility and emission testing, but with considerable skepticism and a general lack of acceptance on the part of many EMC engineers. Reasons given include: 1) a lack of information about interaction effects between the EUT, reverberation chamber, and sources used to excite the chamber, 2) significant unanswered questions concerning the interpretation and accuracy of the measurement results, and 3) the lack of clear correlation of test results with other more conventional measurement techniques. Emphases for performing the work described in this report result from numerous advantages suggested for use of a reverberation chamber. These include:

1. Electrical isolation from or to the external environment;

2. Accessibility (indoor test facility);

3. The ability to generate high level fields efficiently over large test volumes;

4. Broad frequency coverage;

5. Cost effectiveness;

6. Potential use for both radiated susceptibility and emission testing with minor instrumentation changes; and

7. No requirement of physical rotations of the equipment under test (EUT).

These advantages are somewhat offset by limitations which include loss of polarization and directivity information relative to the EMC/EMI profile of the EUT and reduced measurement accuracy. However, this technique does offers a time efficient, cost effective way to evaluate EMC performance of large equipment using existing shielded enclosures with only minor modifications. The concept utilizes the shielded, high-Q, multimoded environment to obtain uniform (time averaged) fields that may simulate "real world", near field environments.

This report describes efforts to answer some of the questions referred to above and outlines an approach using this technique for EMS/V testing. It also describes efforts to identify a "correlation factor" between reverberation chamber and anechoic chamber obtained results, and outlines a detailed, step by step procedure for performing EMS/V tests. An experimental error analysis is given to provide insight into the magnitude of the measurement uncertainties expected for tests performed using this method. 


\section{Development of an Operational Reverberation Chamber}

\subsection{Theoretical Concepts}

A reverberation chamber is a large (in terms of wavelength) high quality ( $Q$ ) cavity whose boundary conditions are continuously and randomly perturbed by means of a rotating conductive tuner or stirrer. The time averaged field inside such a cavity, when a sufficient number of modes are excited, is formed by uniformly distributed plane waves coming from all directions. This property causes the polarization of the field to vary randomly hence eliminating the need, or the utility of physical rotation of test objects in the field. This has its obvious advantages and disadvantages as is apparent from the results and conclusions given in this report.

Two analytical approaches can be used to provide basic knowledge for designing a reverberation chamber. One involves the direct solution of Maxwell's equations with time varying boundary conditions. A formal solution using this direct approach is rather difficult to obtain. In the second approach, suitable linear combinations of basic eigenmodes of the unperturbed cavity (without mode stirrer or tuner) with time-dependent expansion coefficients are taken to represent the field and to satisfy approximately the boundary condition on the surface of the rotating mode stirrer or tuner [8]. The main advantage of this latter approach is that the unperturbed eigenfrequencies and eigenmodes are much easier to calculate, and the problem can be reduced to a more familiar one under special conditions. A necessary condition for the validity of this method is, however, that the total number of eigenmodes which can exist inside a chamber be large for a specified frequency and chamber size. Thus, the measurement technique using reverberating chambers is good for high frequency application. Typical frequencies of operation are from a few hundred $\mathrm{MHz}$ to $18 \mathrm{GHz}$ and above.

The frequencies at which particular modes can exist inside a shielded, rectangular enclosure of dimensions $a, b$, and $d$ are given as [9]

$$
f_{m n p}=150 \sqrt{\left(\frac{m}{a}\right)^{2}+\left(\frac{n}{b}\right)^{2}+\left(\frac{p}{d}\right)^{2}}
$$

$(\mathrm{MHz})$

where $\mathrm{m}, \mathrm{n}$, and $\mathrm{p}$ are integers.

Figure 2.1 gives the distinct frequencies of resonances of the first few modes for the NBS reverberation chamber. There are 26 resonant modes at distinct frequencies below $150 \mathrm{MHz}$ and 63 resonant modes at distinct frequencies below $200 \mathrm{MHz}$.

As expected, the total possible number of eigenmodes, $N(f)$, inside an unperturbed, lossless, rectangular chamber increases in steps with frequency. A smooth approximation to $N(f)$ has been given by $[8,10,11]$ :

$$
N_{S}(f)=\frac{8 \pi}{3} \text { abd }\left\{\frac{f}{c}\right\}^{3}-(a+b+d) \frac{f}{c}+\frac{1}{2}
$$

where abd in $\mathrm{m}^{3}$ represents the chamber volume, $\mathrm{f}$ is the operating frequency in $\mathrm{Hz}$, and $\mathrm{c}$ is the speed of wave propagation in the chamber medium (usually air) in $\mathrm{m} / \mathrm{s}$. Note that the first term in (2) is identical to the well-known weyl's formula derived originally for the same problem by a different approach, and is proportional to the chamber volume and the third power of frequency. The second term is the edge term, which is proportional to the sum of the linear dimensions of the chamber. This term may by used to modify Weyl's result, especially in the lower frequency range. Also, the inner surface area of the chamber, $2(a b+b d+d a)$, is not involved in (2). An example is given in figure 2.2 showing $N(f)$ as curve 1 , $N_{S}$ (f) as curve 2, and Weyl's formula as curve 3 for the NBS chamber. Note that none of the dimensions of the NBS chamber $(2.74 \mathrm{~m} \times 3.05 \mathrm{~m} \times 4.57 \mathrm{~m})$ are equal. Equivalent examples for a square-

based chamber $(2.17 \mathrm{~m} \times 4.19 \mathrm{~m} \times 4.19 \mathrm{~m})$ and a cubic chamber $(3.37 \mathrm{~m})^{3}$, both of which have the same volume as that of the NBS chamber, are shown respectively in figures 2.3 and 2.4 for comparison purposes. Clearly, wider steps for curve 1 in figures 2.3 and 2.4 as compared with figure 2.2 are observed. This is true even though the smooth approximation $\mathrm{N}_{\mathrm{S}}$ ( $\mathrm{f}$ ) $\mathrm{remains}$ almost identical for all the three chambers. The main reason for this is due to the increased mode degeneracy for the last two chambers, under which the total number of distinct eigenmodes with respect to a given operating frequency and chamber size decreases. 
While the total number of eigenmodes inside an unperturbed chamber is an important design criterion, another equally important factor to consider is the mode density function, dN/df, which represents the change in number of modes in a given frequency interval. Ideally, the chamber should be designed, if possible, so that the distribution of the modes is uniform. To determine the exact shape of $\mathrm{dN} / \mathrm{df}$ involves using impulse functions since they are the derivatives of step functions. It is not difficult to see that within a given frequency interval, the average distance betweer the different impulses for the NBS chamber is shorter than that for the other two chambers having the same volume. An alternative quantity to examine for exhibiting this property is

$$
\Delta \mathrm{N}=\int_{\Delta \mathrm{F}} \frac{\mathrm{dN}}{\mathrm{df}} \mathrm{df},
$$

which represents the increase or decrease in mode number within a frequency interval of $\Delta f$.

Results of $\Delta \mathrm{N}$ when $\Delta \mathrm{f}=1 \mathrm{MHz}$ are presented respectively in figures 2.5 through $2.7 \mathrm{for}$ the NBS chamber, the square-based chamber, and the cubic chamber. The uniformity of mode distribution in the frequency interval of $1 \mathrm{MHz}$ is better for the NBS chamber (figure 2.7). This is the reason that the NBS chamber was designed with unequal dimensions. As a consequence, more uniformity for the electromagnetic field is expected within the NBS chamber than for chambers that have two equal sides or that are cubic, with the same volume and frequency of operation. Or, the NBS chamber should be capable of being operated with an extended lower frequency limit than other chambers of the same volume, in order to meet a given degree of uniformity for the final, expected field distribution. Thus, one of the general design criteria for a reverberating chamber is to make the volume as large as possible and the ratio of squares of linear dimensions as nonrational as possible.

A third design criterion, namely, the quality or $Q$ factor, must also be considered in designing a reverberation chamber. Since there are so many eigenmodes which may exist in an unperturbed chamber with each mode carrying its own $Q$ value $[8,10,11]$, it is not always trivial to define a quality factor for the chamber as a whole. A composite quality factor for an unloaded chamber (without an EUT in it) within a specified frequency range can be obtained approximately from the equation [8],

$$
\tilde{Q}=\frac{3}{2} s \delta_{S} \frac{1}{1+\frac{3 \lambda}{16}\left(\frac{1}{a}+\frac{1}{b}+\frac{1}{d}\right)}
$$

where $\mathrm{V}$ is the chamber's volume in cubic meters, $\mathrm{S}$ is the internal surface area in square meters, and $\delta_{s}=\sqrt{\frac{2}{\omega \mu \sigma}}$ is the skin depth in meters, $\lambda$ is the wavelength in meters, and a, b, and $d$, are the chamber's internal dimensions in meters.

The physical meaning of (4) may be interpreted by comparing it with the individual $Q$ values of all the modes in the form of a cumulative distribution. Since $\mathrm{V} /\left(\mathrm{S} \delta_{\mathrm{S}}\right)$ is a common factor whether the composite quality factor defined above or the quality factor for individual modes is considered, it is more convenient to present the results in terms of $1 / Q$ values normalized with respect to $S \delta_{S} / V$. Thus, the variable used herein is

$$
\alpha=\frac{1}{Q}\left(V / S \delta_{S}\right)
$$

Examples of the cumulative distribution of $\alpha$ for the NBS chamber are given respectively in figures $2.8-2.10$ for three different $t$ equency bands. For the frequency band of 180 to $200 \mathrm{MHz}$ in figure 2.8, the total number of modes existing in this band of $20 \mathrm{MHz}$ is 69 , with each mode having its own Q-value. The probability of having a high upper-bound value of $\alpha \leqq$ 0.80 (or a lower-bound for Q) is almost $100 \%$, and that for a low value of $\alpha \leqq 0.48$ (or a high value for Q) is only about $10 \%$. This implies that almost all of the 69 modes in this frequency band have $\alpha \leqq 0.80$. The arithmetic mean of 0.623 and the standard deviation of 0.090 are also indicated in the figure. The probability of having $\alpha \leqq 0.623$ (arithmetic mean) is $50 \%$, meaning that at least one half of the 69 modes have $\alpha \leqq 0.623$. 
For the case presented in figure 2.9, where the frequency band is from 330 to $350 \mathrm{MHz}$, also of a bandwidth of $20 \mathrm{MHz}$, there are 261 modes, an increase in number of modes relative to that in figure 2.8. This is because of higher frequency. A similar interpretation of the $\alpha$ values (or $Q$ values) carried by these modes in terms of probability applies. There are now a small number of modes (low probability) carrying a value of $\alpha$ as low as 0.43 ( $h i g h$ Q). The arithmetic mean and standard deviation are, respectively 0.630 (higher than the corresponding value in figure 2.8) and 0.085 (lower than the corresponding value of figure 2.8). A higher value of arithmetic mean implies that one half or more of the 261 modes carry a higher value of $\alpha$ (lower $Q$ ) compared to the frequency band considered in figure 2.8. A decrease in standard deviation reveals that a greater number of modes have $\alpha$-values closer to the arithmetic mean.

If we consider a still higher frequency band 480 to $500 \mathrm{MHz}$ such as that illustrated in figure 2.10, 534 possible modes will exist in the same bandwidth of $20 \mathrm{MHz}$. The arithmetic mean increases further to 0.646 while the standard deviation decreases further to 0.074 , indicating that a greater number of modes will have still higher $\alpha$ values near the arithmetic mean. This tendency, increasing in arithmetic mean and decreasing in standard deviation with increased frequency, yields a limiting mean of $\alpha \leqq 0.667$ with a 50\% probability, which also agrees precisely with the limiting value for composite $\bar{\alpha}$ derived from (4) and (5).

Thus, even though there are a large number of possible modes existing in a specified operating frequency band for a reverberating chamber, with each mode carrying its own value of $\alpha$ or $Q$, the probability that $\alpha \leqq 0.667$ (or $Q \leqq 1.5 \mathrm{~V} / \mathrm{So} \sigma_{S}$ ) is $50 \%$. This implies that one half of the modes have $\alpha$-values less than 0.667 . Preliminary estimation of a quality factor to characterize the reverberating chamber as a whole, based on the simple expression of $\tilde{Q}$ in ( 4 ), for the purpose of predicting the field strength level to be generated in the test zone is indeed very useful.

Finite $Q$ values also imply that more than 1 mode can be excited at a single frequency. The extent to which modes not specifically resonant at a given frequency are excited is, of course, dependant upon the particular modes' $Q$, and how close the modes' resonant frequencies are to the given frequency.

The composite $\tilde{Q}$ estimated from (4) is considered an upper-bound value because it does not take into account losses other than that due to wall conductivity. In reality, some loss will also occur due to leakage from the chamber, loss in antenna support structures, etc., and loss in the chamber's wall coatings. Hence an alternative means of determining chamber $Q$ can be achieved from measuring the chamber's loss. Chamber loss is determined experimentally by measuring the difference between the net input power, $P_{t}$, delivered to the chamber's transmitting antenna, and the power available, $\mathrm{P}_{\mathrm{r}}$, at the reference antenna terminals. $\mathrm{A}$ photograph showing the interior of the NBS reverberation chamber is shown in figure 2.11. If the energy is uniformly distributed over the volume of the chamber, an empirical value (Q') can be obtained [6] using the equation,

$$
Q^{\prime} \approx 16 \pi^{2} \frac{V}{\lambda^{3}} \frac{P_{r}}{P_{t}} \text {, }
$$

where $V$ and $\lambda$ are as previously defined.

Typical losses for the NBS chamber are shown in figure 2.12. The two curves show the average and minimum loss as a function of frequency. These results were determined statistically by rotating the chamber's tuner through 200 increments of 1.8 degrees and measuring the chamber's loss at each position for all test frequencies. The smooth curves are an estimated curve fit for the data.

Results obtained using (4) to calculate the composite $\tilde{Q}$ and by using the data shown in figure 2.12 and (6) to calculate the experimental $Q^{\prime}$ are shown in figure 2.13. Figure 2.13a gives the curves for the calculated values of $\tilde{Q}$ and $Q^{\prime}$ and figure $2.13 b$ shows the ratio of $\tilde{Q}$ to $Q^{\prime}$. At frequencies above approximately $1 \mathrm{GHz}$ the ratio approaches a constant value approximately equal to 3 or a loss equivalent of approximately $5 \mathrm{~dB}$. 
The $Q$ factor of the chamber, of course, influences the $r f$ input power requirements to generate the EM field levels inside the chamber for performing susceptibility tests. The average power received by the reference antenna, $\overline{\mathrm{P}}_{\mathrm{r}}$ in watts, is related to the average power density, $\bar{P}_{d}$ in watts per square meter, of the EM field inside the chamber by the equation,

$$
\vec{P}_{d}=\vec{P}_{r} / \vec{A}_{r}(W / m)^{2} \text {, }
$$

where $\bar{A}_{r}$ is the average effective aperture in square meters of the receiving antenna.

If the receiving antenna is subjected to EM energy coming from all aspect angles and random polarizations, the average gain of the antenna over $4 \pi$ solid angle is unity. (i.e., The field distribution at each point in the antenna aperture plane is assumed to be a composite of randomly polarized plane waves. This implies that the orientation of the receiving antenna will not influence the measured response and hence the effective gain of the antenna is unity.) The effective aperture of the antenna then is given as, $\bar{A}_{r}=\lambda^{2} / 8 \pi[12]$. However, data obtained, as shown later in this report, indicates that these conditions are not achieved ideally (the field is not completely randomly polarized and incident at all aspect angles and the assumption that the average gain for the antenna is one is not exact). Hence, a more practical value to use for the effective aperture appears to be $\lambda^{2} / 4 \pi$. This value has been suggested previously for use in reverberation chambers for determining the "equivalent" average power density, $\overline{\mathrm{P}}_{\mathrm{d}}[4]$. Equation (7) then becomes,

$$
\vec{P}_{d}{ }^{\prime}=\frac{4 \pi \vec{P}_{r}}{\lambda^{2}} \quad\left(W / m^{2}\right) .
$$

Combining (6) and (8), we can obtain an expression for comparing the average, equivalent power density inside chambers of different size, assuming equal net input power, or

$$
\frac{\bar{P}_{d_{1}}^{\prime}}{\overline{P_{d_{2}}^{\prime}}}=\frac{V_{2}}{V_{1}} \frac{Q_{1}^{\prime}}{Q_{2}^{\prime}}=\frac{S_{2}}{S_{1}} \frac{\delta_{2}}{\delta_{1}},
$$

where the subscripts 1 and 2 refer to the two different enclosures, 非 and 非. For example, assume the $\#_{1} 1$ enclosure is the NBS enclosure with $S_{1}=69.63 \mathrm{~m}^{2}$ and the \#2 enclosure is a larger chamber constructed of the same metal $\left(\delta_{1}=\delta_{2}\right)$ with dimensions, $3.51 \mathrm{~m} \times 5.18 \mathrm{~m} \times$ $10.82 \mathrm{~m}$, or $S_{2}=224.42 \mathrm{~m}^{2}$; this would give a ratio in average power densities (assuming the same net input power) of $\overline{\mathrm{P}}_{\mathrm{d}_{1}} / \overline{\mathrm{P}}_{\mathrm{d}_{2}}=3.22$ (i.e., tre average power density inside the larger enclosure would be approximately $1 / 3.22$ or 0.311 times as much as that in the NBS enclosure). Note that the vaiidity of (9) is dependent upon a number of assumptions alluded to in its derivation.

\subsection{Physical Design Considerations}

A number of criteria should be considered in physically designing a reverberation chamber EM susceptibility/vulnerability measurement system. They can be addressed under the following categories:

1. Chamber physical shape and volume,

2. Chamber quality factor (Q),

3. Chamber auxiliary needs (venting, electrical power, EUT visual monitoring, EUT operational and functional monitoring requirements),

4. Tuner/stirrer(s) design, and

5. Chamber excitation and field monitoring. 


\subsubsection{Chamber Physical Shape and Volume}

This criterion was addressed in Section 2.1. For optimum chamber performance (i.e. spatial field uniformity and accuracy in determining the test field level), especially at the low end of the frequency intended for use, the volume of the chamber should be as large as possible and the ratio of the squares of the chamber's linear dimensions should be as nonrational as possible. Choice of chamber size would be dictated by test volume size requirements, lowest test frequency, and budget considerations.

\subsubsection{Chamber Quality Factor, (Q)}

The second criterion, that of chamber $Q$, is established by carefully considering tradeoff options. If one has the option of designing and constructing a new shielded chamber, it is advantageous to use solid welded aluminum or copper construction since this will result in a higher $Q$ than if sheet steel is used. Presumably one can always lower the $Q$ of the chamber, if desired, by inserting material such as lossy wall coatings or a limited amount of rf anechoic material. The reason for lowering the chamber $Q$ is to increase the bandwidth of the chamber's modes, thus improving mode overlap and smoothing the EM field uniformity vs. frequency response characteristics, especially at the lower test frequencies. Doing this, however, increases the rf input power needed to generate the required field strengths and reduces the effectiveness of the chamber's tuner to redistribute energy uniformly into modes in all three axes of the chamber. In addition, the time required for the chamber test field to arrive at its steady state condition, after the input power to the chamber is applied, is a function of chamber $Q$. The higher the $Q$ the longer the settling or charging time. This could have significant implications if the reverberation chamber is to be used for pulsed $r f$ susceptibility testing. Regardless of the metal used in the chamber's construction (aluminum, copper or steel) it should be standard practice to keep the chamber clear of lossy material such as wood or absorbing materials except for special purposes.

\subsubsection{Chamber Auxiliary Needs}

The third criterion, chamber auxiliary needs, must be established based upon the intended use and the upper test frequency for use. Venting panels should use screens with apertures significantly smaller than waveguide below cutoff at the maximum intended test frequency. Electrical power supplied to outlets inside the chamber, EUT visual monitoring, and EUT operational and functional monitoring requirements are similar to conventional shielded enclosure requirements. A bulkhead panel similar to that shown for the NBS reverberation chamber in figure 2.14 can be used to access the excitation and reference receiving antennas, and the EUT. Care must be taken to ensure that the shielding integrity of the enclosure is not compromised by access holes or cables, etc. This may require the use of high resistance (carbon impregnated) lines [13] or fiber optic links for monitoring the EUT. In addition, waveguides below cutoff access ports can be very useful.

\subsubsection{Tuner Design}

The fourth criterion is to design the tuner to ensure its effectiveness to redistribute the energy inside the enclosure and hence to tune the field. To achieve this, the tuner must be electrically large $(>\lambda / 2$ at the lowest frequency of operation) and be shaped or oriented to distribute energy into all possible modes. Three such tuners are shown in figures $2.15 a$, $2.15 \mathrm{~b}$, and 2.15c. The first tuner (figure 2.15a) was developed by McDonnell Douglas Corp. $[2,14,15]$ after extensive testing to optimize the design for use in their translational electromagnetic environment chamber (TEMEC) facility. The second tuner (figure 2.15b) is used by the Naval Surface Weapons Center in their reverberation chamber [16]. The third tuner ( $f$ igure 2.15c) is used in the NBS reverberation chamber. The tuner is mounted as shown in figure 2.16, with the blades bent at an angle of approximately 45 degrees to the ceiling to improve its scattering of the field evenly in all directions. The tuner mounting includes a shielded housing with internal metal wipers to prevent $r f$ leakage from the chamber via the tuner shaft to the stepping motor. Details of the housing and motor mounting arrangement are shown in figure 2.17. The tuner controller system which includes the stepping motor and a computer accessed controller, allows movement of the tuner in increments as small as 0.113 degrees (3200 steps per revolution) at rates from seconds up to hours per revolution. The motion was optimized for as smooth a motion of the tuner as possible.

A test to determine how effectively the tuner is functioning is to measure the ratio of the maximum to minimum received power of the receiving antenna as a function of tuner position. This is done while maintaining a constant net input power to the enclosure's 
transmitting antenna. The results of such measurements made with the NBS tuner are shown in figures 2.18a and 2.18b. The magnitude of the ratio is dependent upon a number of factors including tuner shape, size, orientation, and the $Q$ of the enclosure. The average value of the maximum to minimum ratio is higher in the empty chamber. (Data for figure $2.18 \mathrm{~b}$ were obtained with 4 pieces of $66 \mathrm{~cm} \times 61 \mathrm{~cm} \times 61 \mathrm{~cm}$ rf absorber placed in the center of the chamber 0.5 meter above the floor). The purpose for inserting the absorber was to simulate a gross loading effect of an EUT placed in the enclosure. A lower loss (ratio) is indicative of a higher $Q$ and more effective mode tuning. This influences the spatial uniformity of the test field (statistically) as is shown in section 2.3. A reasonable guideline for proper operation of the tuner is a minimum tuning ratio of $20 \mathrm{~dB}$.

Multiple tuners have been used in some reverberation chambers $[3,6]$. Typically they are mounted on opposite walls to improve the stirring of the fields in the chamber. This is especially important if the tuners are flat, thus acting as scatterers in only one plane. The tuner controller system used in the NBS system allows for control of several tuners simultaneously. This may be particularly advantageous if the reverberation chamber is especially large or is compartmentalized (multiple sections). Examples of compartmentalized chambers are a large chamber divided into two sections by a separating wall or a small chamber placed inside a large chamber. Both arrangements have been proposed for using the reverberation chamber method for measuring shielding effectiveness of materials and enclosures $[17]$.

\subsubsection{Chamber Excitation and Field Monitoring}

The fifth consideration in designing a reverberation chamber EMC measurement system is in the selection and mounting of appropriate antennas for exciting and monitoring the EM fields inside the chamber. The following parameters are used in selecting the antennas:

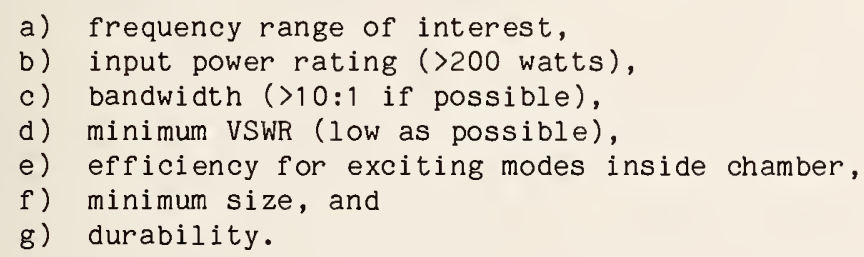

The typical frequency range of application is from $200 \mathrm{MHz}$ to $18 \mathrm{GHz}$ or higher. A number of candidate antennas could be used in this range. No single antenna has sufficient bandwidth to cover the full range with the possible exception of the long wire antenna. Based upon the above and, to a certain extent, the antennas available at the Electromagnetic Fields Division of NBS, the following were selected for evaluation.

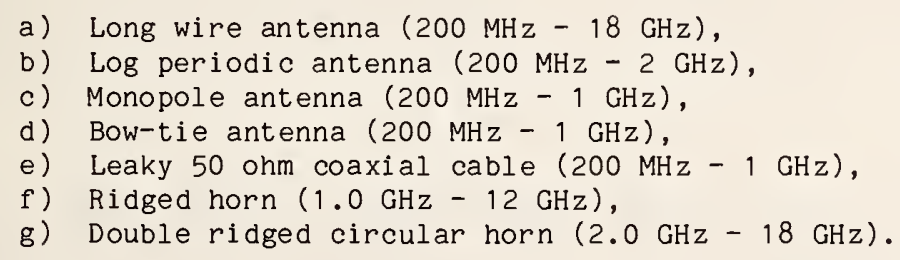

The efficiency with which energy can be injected into or coupled out of the chamber via transmitting or receiving antennas is determined by: 1) the voltage standing wave ratio (VSWR) of the antennas (i.e., the impedance match between the rf source and the transmitting antenna or between the receiving antenna and its output detector), and 2) the ability of the antennas to couple energy into or out of the particular modes that exist at the test frequencies of interest. Rotating the tuner changes the chamber's boundary conditions and hence, shifts the mode excitation frequencies. This, of course, changes the characteristics of the field inside the enclosure which in turn influences the effective VSWR of the antennas. The $Q$ factor of the enclosure as a resonant cavity also has considerable effect upon the VSWR of the antenna. This is particularly true at frequencies below a few GHz.

The placement and orientation of the transmitting and receiving antennas can also influence the operation of the chamber [18]. Two positions or orientations are recommended: 1) position the transmitting and receiving antennas in different corners of the chamber, oriented toward the corners as shown in figure 2.19, or 2) position one of the antennas in a corner oriented toward the corner, for example the reference receiving antenna, with the other antenna (transmitting antenna) sufficiently far away and oriented toward the tuner. The first 
arrangement was selected for use in the NBS chamber. The purpose for these configurations is to couple energy into and out of all chamber modes as efficiently and uniformly as possible without significantly favoring particular modes or favoring a direct path coupled signal between the transmitting and receiving antennas.

Installation of a long wire antenna inside the chamber is different from other antennas because it uses the chamber wall as its ground plane to obtain as close to a 50 ohm impedance as possible. Details for designing the taper transition region of the long wire antenna are shown in figure 2.20. A photograph of the long wire antenna installed inside the NBS chamber is shown in figure 2.21. The long wire extends around two surfaces of the chamber. This is done to achieve, however somewhat limited, the purpose of the locations and orientation configurations referred to above for other conventional antennas.

$G$ Based upon the results of data obtained for various antenna pairs selected from the above list, the following four antennas were chosen for use in the NBS chamber in the following frequency ranges:

1) Long wire (200 $\mathrm{MHz}-1000 \mathrm{MHz})$, receiving mode,

2) Log Periodic (200 MHz - $1000 \mathrm{MHz})$, transmitting mode,

3) Ridged horn (1.0 GHz - $10 \mathrm{GHz})$, transmitting and receiving modes,

4) Double ridged circular horn $(2.0 \mathrm{GHz}-18 \mathrm{GHz})$, transmitting and receiving modes.

Within the recommended frequency bands of operation, the long wire and log periodic antennas can accommodate up to 1000 watts input power, and the ridged horns and double ridged circular horns can accommodate up to 200 watts input power.

A number of preliminary tests were performed to determine the operational parameters of the NBS reverberation chamber using these antennas and to evaluate the interactions between EUT, the chamber, and the $\mathrm{rf}$ sources used in performing these measurements. Some of these tests and their results were previously published [19]. The VSWRs and coupling efficiency of these four antennas placed inside the empty NBS chamber are given in figures 2.22 and 2.23 . Figure 2.22 shows the maximum, average, and minimum VSWR obtained statistically by measuring return loss from the antennas, operating in the transmitting mode, as a function of tuner position at discrete frequencies from $200 \mathrm{MHz}$ to $18 \mathrm{GHz}$. The tuner was stepped at $1.8 \mathrm{degree}$ increments (200 steps) through a complete revolution. Figure 2.22a is for the long wire antenna operating over the complete frequency range. Figure 2.22b, is the composite VSWR of three antennas (非, 非3, and 非) used within the frequency bands shown in the figure's caption. In the past a long wire antenna has been used quite extensively as a reference receiving antenna across the full frequency range $[4,14,17$,$] . Its input VSWR is not as low, especially$ at frequencies above $1 \mathrm{GHz}$, as for the composite of the three antennas. Also, at frequencies above approximately $2 \mathrm{GHz}$, rotation of the tuner appears to have a minor influence on the VSWR of the antennas. This feature can have considerable impact upon the decision as to what approach to use in performing susceptibility measurements as discussed in section 4.1 of this report. Figure 2.23 shows the coupling efficiency or loss between the long wire antenna transmitting (200 MHz - $18 \mathrm{GHz})$, and a composite of the three antennas receiving. This figure is provided for comparison with figure 2.12. The long wire antenna is not as efficient in coupling energy into the chamber and hence, the loss is greater, particularly above $1 \mathrm{GHz}$.

The quality factor of the chamber as a resonant cavity also has considerable effect upon the VSWR and coupling efficiency of the antenna placed inside, especially at frequencies below a few GHz. This is shown in figures $2.22 \mathrm{c}$ and 2.24. These data were obtained with four pieces of $66 \mathrm{~cm} \times 61 \mathrm{~cm} \times 61 \mathrm{~cm} \mathrm{rf}$ absorber placed in the center of the chamber's test zone. Note the significant reduction in the VSWR variations of the transmitting antenna associated with the substantially lower $Q$ in the enclosure and the significant increase in the chamber loss.

\subsection{Determination of Wave Impedance and E-Field Amplitude Distribution Inside Reverberation Chamber}

\subsubsection{Determining the Test Field and Wave Impedance Amplitudes}

The field strength in the chamber can be determined in two ways. The first is to measure the power received by the reference antennas, and then determine the equivalent power density in the enclosure from (8). The equivalent electric field, $\bar{E}_{a}$, is then found using the expression, 


$$
\bar{E}_{a}=\sqrt{\bar{\eta} \cdot \bar{P}_{d}}=\frac{4 \pi}{\lambda} \sqrt{30 \bar{P}_{r}^{\prime}}(V / m)
$$

where $\bar{\eta}^{\prime}$ is the statistically averaged wave impedance in the chamber. This averaged wave impedance is assumed to be approximately equal to $120 \pi$ ohms. Obviously, the wave impedance inside the reverberation chamber will have large variations as a function of tuner orientation and chamber location. To test the validity of $(10)$, independent measurements were made of the maximum, average, and minimum magnetic and electric fields in the chamber. These measurements were made using a one centimeter diameter loop probe and a one-centimeter long dipole probe. The probes were rotated through three orthogonal orientations aligned with the chamber axes and located at the center of the test zone. The magnitudes of the total magnetic and electric fields were then determined as the square root of the sum of the squared values of each of the three components respectively for both the magnetic and electric fields. The corresponding ratios of the electric-field and magnetic-field amplitudes for a data base of 200 positions of the tuner were then used to determine an "equivalent" wave impedance. The results are shown in figure 2.25. Four curves are given. Three curves show the maximum, average and minimum wave impedance determined as a function of frequency. The fourth curve shows the wave impedance calculated from the ratio of $\mathrm{E}$ to $\mathrm{H}$ when the electric-field was a maximum. Even though the wave impedance varies widely as expected with frequency, the average wave impedance at frequencies above $200 \mathrm{MHz}$ (the lower frequency limit recommended for this chamber) is approximately $120 \pi$ ohms. This then, provides evidence that (10) is valid, at least for determining the average E-field in the chamber. The fourth curve was included because, as will be discussed later in this report, the convenient parameter for comparing reverberation chamber with anechoic chamber obtained EUT susceptibility data is the EUT's peak response. This typically corresponds to a maximum electric field in the reverberation chamber. Note that the wave impedance corresponding with the maximum E-field typically is greater than $120 \pi$ ohms and was found to be as large as 1600 ohms thus contributing to an error as large as 6 dB in determining the maximum E-field if (10) is used.

The second method used to determine the field strength in the reverberation chamber is to measure the electric field using an electrically small dipole probe that has been previously calibrated in a standard uniform field. This is the same dipole probe, referred to above, that was used to determine the chamber's wave impedance. The assumption made in using the probe is that since it is electrically small, the fields measured in the reverberation chamber over the aperture of the dipole will be uniform (i.e., "equivalent" to the standard uniform field used in the probe's calibration); and hence the probe's response will give an accurate measurement of the "equivalent" field strength in the chamber. Results of the measurements comparing the electric-field strength generated in the chamber based upon these two methods are shown in figure 2.26. The maximum and average E-field strength data were normalized to one watt net input power. The agreement shown is typical of the random variations in the data used to determine the field in a reverberation chamber. The systematic offset difference between the field determined using the reference antenna and that determined using the probe is, however, less than $3 \mathrm{~dB}$. This result also strengthens the validity of (10).

\subsubsection{Maximum Versus Average Amplitude Responses}

Another important observation from figure 2.26 is the approximate 7-8 dB difference in signal amplitude between the maximum and average field strengths. This can be explained simply in terms of the structure of the cavity modes in the chamber [20]. For a cavity of dimensions, $a, b$, and $d$, modes in all three dimensions can be represented as

$$
\phi_{m n p}(x, y, z) \propto \sin \frac{m \pi x}{a} \sin \frac{n \pi y}{b} \sin \frac{p \pi z}{d},
$$

where $\mathrm{x}, \mathrm{y}$, and $\mathrm{z}$ correspond to the three orthogonal axes of the chamber and $\mathrm{m}, \mathrm{n}$, and $\mathrm{p}$ are the associated mode numbers.

An antenna typically responds to a specific field component, or in the reverberation chamber case, to the composite field impinging upon its aperture. However, measured power is related to $\left.\phi_{m n p}^{2} x, y, z\right)$. The maximum value of $\phi_{m n p}^{2}(x, z, z)$ occurs when each component is a maximum or

$$
\operatorname{Max}\left(\phi_{\operatorname{mnp}}^{2}(x, y, z)\right) \leqq 1 .
$$

The average value of $\phi_{m n p}^{2}(x, y, z)$ is given by 


$$
\text { Ave }\left(\phi_{\mathrm{mnp}}^{2}(\mathrm{x}, \mathrm{y}, \mathrm{z})\right)=\frac{1}{\mathrm{abd}} \int_{\mathrm{V}} \phi_{\mathrm{mnp}}^{2}(\mathrm{x}, \mathrm{y}, \mathrm{z}) \mathrm{dxdydz}=\frac{1}{8} .
$$

The ratio of $\operatorname{Max}\left(\phi_{\mathrm{mnp}}^{2}(\mathrm{x}, \mathrm{y}, \mathrm{z})\right) /$ Ave $\left(\phi_{\mathrm{mnp}}^{2}(\mathrm{x}, \mathrm{y}, \mathrm{z})\right)$ in the limit then is 8 which corresponds to $9 \mathrm{~dB}$ (i.e., this is the theoretical maximum difference that can exist between the maximum and average responses of an antenna or EUT measured in a reverberation chamber). In practice, the mode mixing is not 100 percent efficient to achieve a perfect average nor does one obtain a perfect maximum in all three dimensions. Hence the average difference of 7 to 8 dB shown in figure 2.26 is expected.

In the past, a question regarding the validity of maximum or "peak" reverberation chamber results has been raised. However, this question seems to be resolved as apparent by examining the relationship between maximum and average measurements in the reverberation chamber exhibited in figure 2.26 and from the implications of (12) and (13). These results suggest one can extract one set of data (e.g., average) from the other (e.g., maximum) quite accurately (within $\pm 2 \mathrm{~dB}$ ), assuming of course, that the response of the EUT is $l$ inear.

\subsection{Spatial E-Field Distribution}

Tests were made to determine the uniformity of the maximum and average values of the Efield in the chamber as a function of spatial position and frequency. Seven NBS isotropic probes [21] designed to operate at frequencies up to $2 \mathrm{GHz}$ were placed inside the enclosure as shown in figures 2.19 and 2.21. Each probe has three orthogonally oriented dipoles aligned with the enclosure axes. Measurements were made of the field strength of each orthogonal component at the seven locations for each tuner position (200 steps of 1.8 degree increments). The maximum and average values for each component and the corresponding amplitudes were then determined from the complete data set for a normalized net input power to the chamber of 1 watt. The results of these measurements are shown in figure 2.27. These measurements were made in $50 \mathrm{MHz}$ increments from $100 \mathrm{MHz}$ to $2.0 \mathrm{GHz}$. The spread of the data points show the spatial field variation inside the enclosure at the indicated frequencies. Note at 200-300 $\mathrm{MHz}$ the spread and potential errors in determining E-field amplitude is as great as \pm 8 dB, decreasing to approximately $\pm 2 \mathrm{~dB}$ at $2.0 \mathrm{GHz}$. Note also the large difference in the three orthogonal components at frequencies below $150 \mathrm{MHz}$. This is especially apparent from inspecting figure 2.28 which shows the average values of the seven statistically determined average and maximum E-fields for each component and their composite total determined from figure 2.27. Strong mode structure is indicated in the chamber at frequencies below $150 \mathrm{MHz}$ (i.e., the presence of a dominant mode with its associated directional properties). Hence, there are insufficient modes for proper operation at these frequencies. At frequencies above $150 \mathrm{MHz}$, the relative amplitudes of the field components are approximately the same and the composite total of the average E-field components is approximately 4.8 dB greater than the individual comporients. This indicates that the measured value of the average of each component is independent of polarization of the dipole. (i.e., $\mathrm{E}_{x \text { (ave) }}$ is independent of $\mathrm{E}_{\mathrm{y}(\mathrm{ave})}$, etc.). Thus,

$$
E_{t(a v e)}=\sqrt{\left(E_{x(a v e)}\right)^{2}+\left(E_{y(a v e)}\right)^{2}+\left(E_{z(a v e)}\right)^{2}} \approx \sqrt{3}\left(E_{(x, y, o r ~ z) a v e}\right) \text {. }
$$

The composite total however, of the E-field components' maxima (figure 2.28b) is less than $4.8 \mathrm{~dB}$. This indicates that the maximum measured values for each component are not

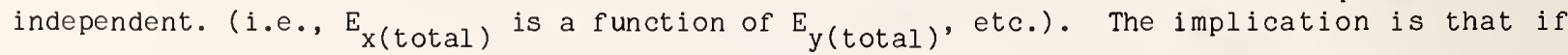
multiple receptors are involved in establishing the maximum susceptibility of an EUT (or for example in measuring the E-field in the chamber by using an isotropic probe with 3 orthogonal dipoles), the difference between the maximum and average response may be less than the typical 7 to $8 \mathrm{~dB}$ referred to in section 2.3 .

\subsection{Multiple Excitation of the Chamber}

Electromagnetic fields were generated inside the chamber using two separate antennas simultaneously excited in the frequency ranges, (200-400) MHz, and (1000-2000) MHz. The antennas used for the frequency range (200-400) $\mathrm{MHz}$ were a log periodic and a long wire described previously. Two ridged horns were used in the frequency range (1.0-2.0) GHz. The spatial, statistical, E-field uniformity did not improve significantly over using a single antenna excitation in the 200-400 $\mathrm{MHz}$ frequency range; however, it did improve slightly (from approximately $\pm 2 \mathrm{~dB}$ to $\pm 1.5 \mathrm{~dB}$ variations) in the frequency range $(1.0-2.0) \mathrm{GHz}$. 
The E-field amplitude in the chamber decreased when using two excitation antennas, in both frequency ranges, assuming the same net input power as for a single antenna. The decrease was approximately $1 \mathrm{~dB}$ in the frequency range (200-400) $\mathrm{MHz}$ and approximately $1.5 \mathrm{~dB}$ in the frequency range $(1.0-2.0) \mathrm{GHz}$. This loss however, can be accounted for in the losses in the additional rf transmission line cables and the second excitation antenna (i.e., the antennas are not $100 \%$ efficient in converting input rf power to radiated power).

3. Interaction Effects Between Chamber, Source and Reference Antennas, and EUT.

\section{1 Placement Constraints of EUT}

Constraints on the placement of EUT inside the reverberation chamber are a result of the proximity effect of the chamber walls on the test field and upon the EUT. Measurements were made of the E-field close to the chamber's walls for comparison with measurements made of the E-field within the test zone outlined by the matrix of seven probes shown in figure 2.21 Two locations were selected: 1) $0.5 \mathrm{~m}$ from the chamber's side and back wall, $1.0 \mathrm{~m}$ above the floor; and 2) $0.12 \mathrm{~m}$ from the side wall approximately mid-way between the chamber's ends and $0.5 \mathrm{~m}$ above the floor. Location 1 was selected so the EUT test zone would be clear of obstructions but also with the probe far enough from the chamber's walls, hopefully, to avoid significant proximity effects. Location 1 measurements were consistent with the measurements made at the 7 probe locations. Location 2 was selected to determine just how significant the proximity effect is at distances less than one third wavelength ( $<800 \mathrm{MHz}$ ). The average Efield measured at location 2 was approximately $2 \mathrm{~dB}$ lower at frequencies from 200 to $400 \mathrm{MHz}$ than the E-field measured in the test zone. The field close to the chamber wall then increased as a function of frequency, approaching the test zone measured field strength at frequencies above $1 \mathrm{GHz}$. These results indicate a need to maintain a minimum spacing for the reference antenna, field measurement probe(s), and/or EUT of at least $\lambda / 3$ from the chamber's walls at the lowest test frequency. This equates to approximately $1 / 2 \mathrm{~meter}$ at $200 \mathrm{MHz}$ for the NBS chamber. An exception to this guideline may be a desired spacing between the EUT and the chamber floor or walls. This spacing may be dictated by the test plan. If the EUT is electrically large (many wavelengths) it may be necessary to have a separation distance from the chamber walls greater than $\lambda / 3$ at frequencies where $\lambda / 3$ is less than $1 / 2$ meter or greater than $1 / 2$ meter under some test conditions to prevent shadowing effects from influencing the spatial distribution of the test field. Results of scattering effects measurements discussed in section 3.2 suggest shadowing is not a signficant problem, if the EUT is placed in the center of the test zone as far from the walls as possible.

\subsection{Scattering Effects of Metal Objects upon E-field Distribution}

Two test objects were selected to simulate an EUT placed in the chamber to evaluate their scattering effect upon the field distribution inside the chamber. The first object was a solid welded aluminum box $30 \mathrm{~cm} \times 50 \mathrm{~cm} \times 60 \mathrm{~cm}$ in size. The second was an electronic equipment rack $56 \mathrm{~cm} \times 67 \mathrm{~cm} \times 114 \mathrm{~cm}$ in size. Tests were made to determine the E-field uniformity or distribution over the test zone matrix defined earlier using 6 isotropic probes (figure 2.19) with each of the two test objects placed at the center of the test zone. (The probe at the center of the test zone was removed to accommodate the test objects.) Figures $3.1 \mathrm{a}$ and $3.1 \mathrm{~b}$ show the two test objects placed inside the enclosure. The mean value of the maximum and average values for each E-field component (vertical, longitudinal, and transverse) and their composite total were determined for each of the 6 probes. The results, shown in figure 3.2, give a comparison with the empty chamber E-field distribution measurements shown in figure 2.28. Little or no difference was noted in the statistical (average and maximum) values measured.

\subsection{Loading Effects of EUT on Chamber}

One of the concerns expressed about using a reverberation chamber for EMC measurements was the realization that if the EUT absorbed energy from the chamber (i.e., demonstrates susceptibility) it would reduce the $Q$ of the chamber and thus affect the measurement results. Typically this is compensated for by simply increasing the net input power to the chamber transmitting antennas as required. This may not be sufficient if inserting the EUT lowers the chamber's $Q$ to the extent that the chamber no longer reverberates or the tuner will no longer sufficiently stir the modes, (i.e., the chamber no longer functions properly). Recall that the absolute amplitude of the test field level inside the chamber is determined from the reference antenna received power measurements. The assumption is that this measurement is unaffected by the loading effect of the EUT. Two simple tests were used to evaluate the loading effect: the first was to insert a $500 \mathrm{MHz}$, half-wave, resonant dipole near the center of the chamber's test zone to determine if energy coupled from the chamber to the dipole would 
reduce the field strength in the chamber. This test was intended to simulate the loading effect of a simple resonant EUT. Field strength measurements were made again using the seven probe matrix and the long wire antenna as a reference receiving antenna to determine the level of the test field, before and after inserting the resonant dipole. A constant normalized input power of 1 watt was applied at the input of the chamber log periodic transmitting antenna to establish the field in the chamber. The following observations were made:

1) Energy is conserved (i.e., power coupled from the chamber via the dipole results in a proportional decrease in the E-field in the room).

2) The $500 \mathrm{MHz}$ resonant dipole and the long wire receiving antenna coupled approximately the same amount of power from the room.

The seven probe matrix used to determine the spatial distribution of the E-field inside the chamber, also load or reduces the $Q$ factor in a similar fashion to the resonant dipole. Each of these probes couple a small amount of energy out of the test field. In addition, they have lossy transmission lines made up of carbon loaded teflon that serve to convey their detected de signals to their instrumentation located outside the chamber. These lossy transmission lines increase the chamber's loss up to $5 \mathrm{~dB}$ at $2.0 \mathrm{GHz}$. However, this amount of loss did not seem to significantly influence the ability to determine the field level in the chamber, with or without the presence of the probes by using the reference receiving antenna. The field level simply was lower for the same net input power due to the loading effect of the probes in the chamber.

The second test used to evaluate the loading effect was to place varying amounts of $r f$ absorbing material inside the chamber. This was done to get an indication of just how much loading the chamber could tolerate before it fails to operate properly and/or large errors existed in determining test field level and EUT response. Progressively greater amounts of absorber were added until approximately 90 percent of the net input power was being absorbed (i.e., the chamber loss was increased by approximately $10 \mathrm{~dB}$ ). The amount of $\mathrm{rf}$ absorber required was four pieces of $66 \mathrm{~cm}$ pyramidal high performance absorber $61 \mathrm{~cm} \mathrm{x} 61 \mathrm{~cm}$ square. Results for tuner effectiveness, VSWR and chamber loss with absorber loading were shown in figures 2.18b, 2.22c, and 2.24. As indicated earlier, lowering the chamber $Q$ reduces the test field level in the chamber for a given net input power. Inserting the 4 pieces of absorber in the chamber lowers the field considerably as shown in figure 3.3. This figure compares the maximum E-field strength in the test volume defined by the 7 probe matrix inside the empty chamber with the field strength after the chamber is loaded with the 4 pieces of absorber.

In summary, the following observations can be made from the data obtained (figures $2.18 \mathrm{~b}$, 2.22c, 2.24 and 3.3). Lowering the chamber Q:

1) decreases the effectiveness of the tuner (lower Max/ in ratio),

2) improves the VSWR of the transmitting and receiving antennas,

3) increases the chamber loss and hence increases the rf power required to obtain test fields of the same level,

4) increases the uncertainty in determining the test field level and EUT response (indicated by the larger variations in the E-field data obtained with absorber, figure 3.3), and

5) decreases the spatial, statistical E-field uniformity.

In conclusion, the following guidelines are suggested. Inserting the EUT should not lower the maximum or average received power from the chamber's reference antenna for the same net input power into the chamber (before and after inserting the EUT) excessively. If these ratios, net input power/received power(max or ave), increase more than $6 \mathrm{~dB}$, or if the tuner's average effectiveness decreases to less than $20 \mathrm{~dB}$, use of the reverberation chamber is not recommended.

\section{0 i.rforming Immunity Measurements}

\subsection{Planning the Measurements}

A number of considerations are important in planning the measurements, writing the test plan, and documenting the test results. One is the amount of data required to accurately 
characterize the EUT. Equipment susceptibility to EMI is primarily determined by the degree to which the interference field couples into and interacts with the equipment's components. This undesired coupling is influenced by a number of equipment parameters such as: input/output, power line, and circuit lead impedances and lead lengths; impedances of circuit components (especially those terminating lead wires); type of circuit components (particularly active components); and amount and type of EMI shielding and filtering used. The susceptibility of any particular equipment is usually a function of frequency, suggesting resonance effects within the equipment with its input/output leads and other interconnected equipment. These resonances may be caused, for example, by the reactance of the connecting leads acting as an antenna, coupling with the input impedance of the terminating circuit components. The quality factor ( $Q$ ) of such a resonant circuit determines the maximum spacing or increment between frequencies at which susceptibility tests must be performed. Automated systems such as required by the reverberation chamber test methods are (by their digital nature) discrete frequency systems. The number of test points one can obtain using such a system are limited by the memory or storage capacity of the computer, the measurement system bandwidth, and the measurement time constraints. Thus, care must be exercised in choosing frequency and amplitude measurement intervals compatible with the test system and the number of test points required.

A second consideration is the test-field time duration versus reaction time of the EUT. Some EUTs have components with relatively slow thermal time constants. This equipment must be exposed to test fields with sufficient duration to allow reaction and interaction; otherwise susceptibility tests may not be true indicators of the equipment's vulnerability. For example, thermocouple devices used to simulate electro-explosive devices may require one to two seconds exposure time for maximum response. EUT soak time requirements influence the approach (section 4.2) selected for performing the tests using the reverberation chamber method.

Another important consideration relates to the need to simulate as closely as possible "real world" operating conditions. Ideally, susceptibility tests should be performed with the EUT connected and operated in its operational environment. Since this, most likely, is not possible, these operational conditions should be carefully simulated. This normally includes making certain the EUT is tested with its wiring harness in the same configuration (as nearly as possible) as when in actual use. (Actually, one of the advantages of the reverberation chamber method is that the test results are independent of EUT wiring harness, input/output and power lead orientation.) In addition, all connecting leads should be terminated with equivalent impedances as when in actual use. Failure to simulate these conditions can result in data that are misleading. An additional problem is the EUT-antenna-enclosure interaction that exists inside any shielded enclosure. Antenna radiation or receiving characteristics are altered when enclosed inside a shielded environment. One should realize that the EUT always interacts somewhat with the exposure field in any environment. What is difficult is sorting out the normal open-field scattering effect from the unknown perturbation effects of the EUTantenna-enclosure interaction that is typically ignored in shielded room susceptibility testing. Efforts to quantify this effect for reverberation chamber obtained results are discussed in section 5 .

How to sense and telemeter the performance of the EUT from the test environment without perturbing the EUT, the test environment or sensitive test equipment is another important consideration. This is usually done by using either fiber optics lines or "invisible" wire [13] (carbon impregnated Teflon) with appropriate readout devices.

Finally, one obvious purpose for performing susceptibility tests is to determine EUT compliance with pre-established performance criteria. For production item sampling, a worst case EMI exposure environment with pass-fail criteria may be appropriate. However, for engineering development of EMI-hardened equipment or for correcting EMI problems discovered in existing equipment, diagnostic testing is needed. This type of testing requires knowledge of the exposure field parameters, such as: frequency, repetition rates, and wave shape of test signal; type and percent of modulation imposed; and polarization, amplitude and signal waveform time variations. These parameters must be carefully selected, as part of the test plan, controlled, and (for some tests) statistically characterized for the testing to be meaningful. One obvious limitation of the reverberation chamber method for susceptibiltiy testing is the loss of polarization information. However, this is in part compensated for because worst case response characteristics of the EUT can be determined without repositioning or orienting the EUT in the reverberation chamber since the test field is rotated around the EUT. If carefully controlled tests are performed, resonances and susceptible EUT circuit components can be determined, from which cause and effect relationships can be established. These can then be modified or eliminated to improve the immunity of the EUT. Another 
important consideration in performing diagnostic testing is the desirability of determining how the EMI is coupled into the EUT. This requires separating, if possible, the sources of interference reception, such as EUT input/output and power line leads, leakage through the EUT case housing, or internally generated and coupled EMI. This can be achieved by a systematic process of shielding and filtering various leads and functional sections of the EUT's circuit, etc., while conducting the susceptibility tests.

\subsection{Measurement Approach (Tuned vs. Stirred)}

Two approaches are used for performing EMC measurements using the reverberation chamber. The first approach, mode tuned, steps the tuner at selected, uniform increments, permitting measurements of the net input power, reference antenna received power, field measuring probe response and the EUT response at each tuner position. This allows corrections and normalization of the received power, field measurements and EUT response to be made for variations in the net input power to the chamber. These variations are due to changes in the input VSWR of the transmitting antenna as a function of the tuner position. The number of tuner steps per revolution that must be used is a function of frequency and chamber $Q$. Typically, at least 200 steps or more are required to provide sufficient sampling to accurately determine the statistical parameters of the test field and EUT response (maximum and average amplitudes). If the sample size is too small, the fidelity of the measurements suffers and true maxima, minima, etc. are not recorded. The mode tuned approach also allows the operator to select long test field exposure times as needed to accommodate some EUT response times.

The second approach, mode stirred, rotates (steps) the tuner continuously while sampling the reference antenna received power, field probe response and EUT response at rates much faster than the tuner revolution rate. These measurements are made using a spectrum analyzer, diode detectors, and "smart" voltmeters with their own microprocessors capable of data storage and calculation of statistical functions such as mean values and standard deviations. The mode stirred approach allows large data samples (up to 9,999) for a single tuner revolution. Tuner revolution rates are adjusted to meet EUT response time and output monitor response time requirements. Typical revolution periods are from 1 to 12 minutes. This large sample, as compared to mode tuned, improves the accuracy in determining the statistics of the measured parameters, however, at the expense of increased uncertainty due to failure to correct for net input power variations.

Hence a trade-off exists between mode tuned and mode stirred operation. An examination of figure 2.22 suggests this trade-of $\mathrm{f}$ occurs approximately between 1-2 GHz. (i.e., The mode tuned approach would be more accurate below 1-2 GHz where the mode density is not as great and corrections can be made for variations in the net input power caused by large variations in the input VSWR of the transmitting antennas. Mode stirring is more accurate at frequencies above 1-2 GHz where VSWR variations in the transmit antenna as a function of tuner position are small and large data samples of the test field and EUT response can be taken.)

\subsection{Measurement System}

\subsubsection{Measurement Setup and Instrumentation Requirements}

A block diagram of the basic NBS reverberation chamber EMC measurement system is shown in figure 4.1. The test field is established by means of rf source(s) connected to transmitting antenna(s) placed inside the chamber. Antennas used are discussed in section 2.2.5. Modes excited inside the chamber are then tuned or stirred by rotating the mode tuner which functions as a field-perturbing device. The EUT may be placed anywhere convenient within the chamber provided no point on the EUT is closer than $1 / 2$ meter to any wall, or ceiling. Placement relative to the floor is dependent upon a number of factors including intended use configuration relative to ground-planes etc., and should be specified by the test plan. Test, power, and control cables are routed to appropriate monitors, etc., outside the chamber via shielded or filtered cables and feed-through connectors, high resistance lines, and fiber optic lines as required to prevent leakage of fields to the outside environment. Note that a precision $10 \mathrm{~dB}$ attenuator is used with the power detector or spectrum analyzer, whenever possible, to measure the receiving antenna power. This is done to minimize impedance mismatch with the receiving antenna. The calibrated bidirectional coupler at the input to the transmitting antenna allows measurements of the net input power so corrections can be made for changes in net input power resulting from antenna-rf generator impedance mismatch and $r$ generator output variations that occur during the rotation of the tuner. As referred to earlier, these corrections are made when using the mode tuned measurements approach for each incremental change in tuner rotation. For mode stirred, the net input power is measured only 
at the beginning of the measurement cycle. Details of how the measurement cycle proceeds under computer control, how the data are managed, recorded, and processed for presentation are contained in the next section describing the software used for these functions.

\subsubsection{Software Requirements and Measurement Procedures}

The computer code used in the evaluation of the reverberating chamber has evolved from simple routines that record only the measurement data to the present complex programs that have abilities to recover from many common system failures and operator errors. The more sophisticated programming is made possible by using modern laboratory computers containing large amounts of memory. The primary function of recording the measurement data however, remains unchanged and is outlined in this section, with some discussion of the supporting activities and refinements.

A general overview of the software is shown in figure 4.2. The major tasks of accumulating data using the two primary methods (mode tuned and mode stirred), saving the measured results, and preparing the data graphics are shown. The measurement routines are supported by the module entitled system Calibrations. The data library is not actually program code, rather it indicates data storage that is available to the Data Graphics program and is added to by the measurement programs. Two major programs, detailed below, are used to implement the measurement approaches. They are quite different in how the instrumentation is programmed and the data managed and will be discussed separately. The basic computer code defining these measurements is listed in appendix A. The listings contain the details of the measurements as shown in figures 4.3 and 4.4. The instrument control modules have been omitted to avoid excessive detail.

Mode-Tuned Approach

The flow diagram for the mode-tuned measurement program is given in figure 4.3 . This diagram is used to assist in guiding the operator systematically through the measurement procedure.

Step 1. Place the EUT inside the chamber and access at as required for operation and performance monitoring.

Details for placement of the EUT and accessing it are contained in sections 3.1, 4.1, and 4.3.1. The EUT should be placed at least one half meter away from the outer walls of the chamber unless specified in the test plan to simulate proximity to a ground plane.

Step 2. Connect the measurement system as shown in figure 4.1 .

Step 3. Specify measurement and calibration parameters.

Calibration factors for the cables and directional couplers used must first be tabulated and stored in the computer for later access. All parameters used in the measurements are then specified using a menu of items from which the operator may select the values for the experiment. These parameters include: frequency range and increments, number of tuner steps, signal generator output level as required to obtain the desired field strength inside the chamber, the EUT output voltage considered as an upper bound for maximum safe EUT operation, and an estimate of the response time of the EUT. The experiment description, operator's name, and the system clock can be accessed in the menu also. When the operator is satisfied with the parameter values and exits the menu, these values plus all calibration values (e.g., cable loss, coupling factors, etc.) are tabulated for reference. The measurement hardware is then initialized and the measurement begins.

Step 4. Perform the measurement (automated measurement sequence).

The measurement sequence can be traced in figure 4.3. At the beginning of each test all the instruments are set to zero (if there is an internal auto-zeroing feature) or a reading is taken to determine the offset from zero with the field removed. These offset readings are then used to adjust the readings taken with the field applied. This should be done as often as needed to minimize errors resulting from zero drift.

It is important to have reached both mechanical and electrical stability at each step of the tuner before reading the instruments. As the mechanical time constant of the stepping motor and the chamber's tuner blades is typically much longer than the electrical response time of the measurement instrumentation or the EUT, the most efficient manner with which to 
collect the data is to minimize the movements of the tuner. Any given measurement usually involves a sequence of frequencies at which to measure the response of the device. This routine is designed to collect the data for all frequencies at each tuner step before moving the tuner to another location. The data is then rearranged at the conclusion of the measurements such that data from all tuner positions for a given frequency is together before it is processed for final graphic presentation.

After the instruments are zeroed and their offset voltages recorded, the rf power is applied and the response of all devices which could be damaged by excessive field strength is measured. This checking is done as rapidly as possible for each frequency and provides one level of protection for the system. Typically, the operator should specify power levels that are appropriate for the EUT(s) being tested. Then this test will detect only those conditions which cause an unusually high response.

With the initial checking done, the program then proceeds to read the remaining instruments in the system. These include power meters, digital voltmeters, spectrum analyzer, specialized data acquisition systems, and/or any other monitoring device being used.

As mentioned above, all frequencies of interest are measured before the tuner is moved to the next position. There are many possible ways to group this data and save it on a mass storage device. The approach used here is to maintain all the data for a given tuner step (or several steps) together in one file. This file can then be recalled and the data rearranged such that all the information for a given frequency is together. Saving the data frequently allows the measurement to be continued, without starting over, should the system lose power or a fatal error occur.

The routines necessary for operation of the instruments are, of course, unique to the device but some general concepts are useful to discuss:

a). The program should exercise some care to insure the validity of the readings. Most instruments wiil allow access to a status byte that will indicate many failures common to the device. This can be monitored during the read cycle and action taken if the measurement is not valid. Action is also necessary if the device fails to respond or hangs the interface bus. These situations may require operator assistance, but the program should be able to recover and repeat the operation if the condition is temporary.

b). Readings taken within the limits of the instrument yet too quickly for the rest of the system to respond, for instance the tuner may still be vibrating or the. sensor may be slow to respond, will cause erroneous results. For these measurements where the field being monitored is generated by a cw signal of constant amplitude, the response of a device should settle to a constant value, provided the tuner has stabilized and the device has had enough time to respond. The computer should be able to determine when to accept the reading based on the factors mentioned. One method is to install fixed wait statements that guarantee steady conditions. A more dynamic approach is to monitor the device and compare readings until steady conditions are reached. This optimizes the already lengthy measurement by avoiding long idle times that may not be necessary. A refinement to simply comparing readings is to compare the averages of several readings grouped together, referred to as a running average. This has a tendency to smooth noise that may be on the signal and also to make comparisons of low level readings (near the noise floor) more stable.

Step 5. Correct the data by applying the system calibration values and zero field offset readings.

The correction factors are applied to the measured quantities at the time the data are taken and key items are calculated. These items are then stored in the memory for later use in compiling the data. Items may include incident and reflected powers to the transmitting antenna corrected for cable loss and coupling factors, power received from the reference antenna (corrected), etc. Care must be taken at the beginning of the measurement to assure that calibration factors and cable loss correction to be used match the physical setup.

At the completion of the last tuner position, with all the data residing in mass storage, the measurement routine is complete. The remaining tasks are independent programs, freeing the measurement system for other measurements.

Step 6. Compile the corrected data by frequency, normalize to a constant net input power (for example 1 watt), perform the statistical calculations and present final results. 
Normalization of the corrected measurement data for determining: a) the E-field in the chamber, and b) the EUT response, for a constant net input power is done for each tuner position. First the corrected net input power is determined and compared to the desired normalized value. The difference is then used to shift the corrected received power measurements and EUT response measurements as if the actual net input power had been the desired normalized value. This may involve a simple linear extrapolation as is the case for linear detectors, or it may require calibration of the nonlinear response characteristics of the received power detectors and/or EUT monitors thus resulting in appropriate corresponding nonlinear corrections.

The statistical calculations are performed using the corrected, normalized data. These calculations include the maximum, average, and minimum values as appropriate, of the desired parameters (net input power, received power, EUT output response, etc.). The calculations are made using data sets obtained by using the corrected normalized data at the selected number of descrete tuner positions for one complete revolution of the tuner, at each frequency of interest. These result are then, typically, presented in graphs as a function of frequency.

Mode Stirred Approach

The mode-stirred measurement (figure 4.4) begins in a similar manner to the mode-tuned discussed above. The automated measurements sequence of events is somewhat different and hence some of the parameters necessary for performing mode-stirred measurements differ from mode-tuned.

Step 1. Place EUT inside the chamber and access it as required for operation and performance monitoring.

This is the same as for mode tuned.

Step 2. Connect the measurement system as shown in figure 4.1

This step is similar to the setup for mode tuned except the spectrum analyzer is used to measure the reference antenna received power and the EUT monitors must be fast sampling devices such as "smart" voltmeters referred to earlier.

Step 3. Specify measurement and calibration parameters.

These parameters are similar to the mode tuned case with the following two exceptions:

a) The specification of revolution time for the tuner determines the sample size and sampling parameters associated with the EUT monitoring equipment. Tuner steps (sampling size) are now given as a function of time for one complete revolution of the tuner from which the number of samples that will be taken during that single revolution, for each of the sampling instruments, is determined. These numbers require careful timing trials prior to automating a mode-stirred measurement. The instruments used for the tests detailed in this report, "smart" digital voltmeters, are capable of performing statistical operations on a large number of samples (up to 9999) using a single trigger command. This feature is very convenient for this type of measurement but requires that the number of samples needed to synchronize rotation time of the tuner and total sampling time of the voltmeter be known prior to triggering the measurement. The time required for taking each sample voltage varies slightly with the amplitude of the signal measured. This makes it diffisult to estimate accurately the total time required for any given number of samples. Approximations are used and the program adjusts the sample count to insure the correct sampling time for a complete revolution. The sampling should be completed as near to the end of the tuner revolution as possible to avoid weighting the statistics with an incomplete data set or data taken as the tuner begins a second revolution. Typical rates for tuner rotation are in the range of 1 to 12 minutes per single revolution.

b) The signal generator output level is now a range of values because this program has the ability to search for an optimum response of the device. The range of response values is also accessible in the mode-stirred menu and gives the operator control over minimum readable output from the EUT and also the level considered as the upper safe limit. The program will attempt to keep power levels adjusted such that the response is at some point above the minimum and never above a maximum. 
Sometimes it is advantageous to monitor the EUT response using analog devices such as an xy recorder. If care is taken to insure that the response time of the recorder is short compared to the sampling rate or rotation rate of the tuner, the EUT output can be monitored in real time, thus allowing optimization of the sample rate to correspond to the EUT response time. Care must be taken to insure adequate sample time, otherwise the EUT response will be clipped yielding inaccurate results. This condition is easy to detect from the analog output plots and hence avoid.

When the operator is satisfied with the parameter values and exits the menu, these values plus all calibration values (e.g., cable loss, coupling factors, etc.) are tabulated for reference. The measurement hardware is then initialized and the measurement begins.

Step 4. Perform the measurement (automated measur ement sequence)

The measurement sequence is shown in figure 4.4. At the beginning of each test all the instruments are set to zero ( $r f$ power is off), if possible, or the zero offset is recorded the same as for the mode-tuned approach. The $r f$ power is then applied at the lowest specified level, the tuner motion is activated, and the EUT response is monitored. This first measurement attempt, at each specified frequency, results in a search for a threshold response from the EUT. If the EUT's response is below the preselected level, the $r f$ power is increased in $1 \mathrm{~dB}$ increments and held constant allowing the tuner to rotate a few degrees, while searching for the threshold level. If the threshold value for the EUT's response is not reached, the generator level is increased again and the searching process repeated until the threshold level is reached or the maximum range selected for the generator output is achieved. Upon finding an acceptable power level, the instruments are triggered to begin a measurement cycle. Typically the instruments used include "smart" digital voltmeters as mentioned earlier and a spectrum analyzer looking for a peak response from the reference antenna. Any specialized detecting device would also be included. The response of the EUT is constantly monitored via a second fast sampling voltmeter (in addition to the "smart" voltmeter doing the statistics) to protect it from overload. If, during the test, the response should exceed the maximum safe value so that the power level is decreased or any other error condition is detected, the program will remember the correct power level to be used as the measurement is restarted.

At the time all the instruments are done sampling, the program checks to see if the tuner completed a full revolution before the measurements (sample) were finished. A comparison of the times taken for the tuner revolution and each meter's total sampling time is made. If these values agree to within a given tolerance and no other errors were detected, then the numbers are assumed to represent a valid measurement. On the other hand, if these conditions are not met, the sampling parameters are adjusted and the entire measurement process is repeated for the same frequency. Special care taken in the preparation of these sample parameters, as mentioned earlier, will minimize the need for repeat measurements.

The results from all the instruments are now read into the computer, corrected using the appropriate calibration factors and the necessary calculations are made. This includes the statistical parameters for the EUT monitored data, as determined by the "smart" voltmeters, and the peak value of the received power from the reference antenna as measured using the spectrum analyzer. Caution must be exercised if the test field is modulated since this may influence the accuracy of the measurement results depending upon the detector(s) response characteristics.. The reference antenna received power data can be used to determine a normalized response for the EUT based upon a constant test field level as a function of frequency if desired. The net input power delivered to the chamber is measured after the acceptable power level is established at the beginning of the measurement cycle, just prior to triggering the "smart" voltmeters. These measurements may then be used to normalize the data to an equivalent constant input power level preselected for all test frequencies if desired. The entire process is then repeated until all frequencies have been measured.

The process by which the statistics are derived for the parameters monitored by the "smart voltmeters" consists of the following steps: 1) the measured analog data is converted to digital form; 2) the first data value is stored for future reference; 3 ) the second and subsequence data values are compared to the previous data values to determine maximum, minimum, and the running total values; 4) at the conclusion of the data collection, (completion of the data sample) the running total is divided by the sample size to calculate an average value for the data set; and 5) finally, the maximum, minimum, and average values stored in the "smart" voltmeter are transferred upon command to the computer for mass storage and future use as required. 
Step 5. Compile the corrected data and present final results.

Data obtained using the mode stirring approach is not normalized at each tuner position. Corrections applied to this data to normalize it to a specified value of net input power must be applied to the maximum, average, and minimum values as determined by the "smart" voltmeters (EUT and other monitored responses) or the maximum value (reference received power) as determined by the spectrum analyzer. Correction made to the data for cable loss, etc., must also be made in a similar way. After these corrections are made, the data is compiled by frequency and presented typically in graph form usually showing only the maximum values as a function of frequency. For example see figures 5.15 and 5.16 .

5. Experimental Test Results

\subsection{Correlation of Results to Anechoic Chamber Tests - Some Examples}

This section describes efforts to estimate a "correlation factor" between reverberation chamber and anechoic chamber obtained results. This was done first for reference standard EUTs and then for an EUT more typical of operational equipment. Tests were performed using the NBS reverberation chamber and a $4.9 \mathrm{~m} \times 6.7 \mathrm{~m} \times 8.5 \mathrm{~m}$ anechoic chamber also located at NBS. The EUTs included in this study are: 1) a one centimeter dipole probe antenna (200 MHz to $18 \mathrm{GHz}$ ), 2) a $\mathrm{ridged}$ horn antenna ( $800 \mathrm{MHz}$ to $10 \mathrm{GHz}$ ), 3) a series of rectangular coaxial transmission line (TEM) cells with apertures, (200 $\mathrm{MHz}$ to $4000 \mathrm{MHz}$ ), and 4 ) a modified $7.0 \mathrm{~cm}$ $(2.75$ ") folded fin aircraft rocket, (FFAR) (200 $\mathrm{MHz}$ to $12.0 \mathrm{GHz})$.

\subsubsection{Description of Anechoic Chamber Measurement System}

A block diagram of the NBS anechoic chamber EMC measurement system is shown in $f$ igure 5.1. The test field is established inside the chamber by means of an $r f$ source connected to a standard gain transmitting antenna. This "standard field" is computed from [22]

$$
E=\frac{\sqrt{30 \mathrm{P}_{\text {net }} \mathrm{G}}}{\mathrm{D}} \text {, }
$$

where $P_{\text {net }}$ is the net power in watts delivered to the transmitting antenna, $G$ is the effective gain for the transmitting antenna, and $D$ is the separation distance in meters. Equation (14) assumes far-field conditions for a field point on axis of the transmitting antenna so that $G$ is the maximum power gain.

The net power, $P_{\text {net }}$, is determined using calibrated power meters and bidirectional couplers from the expression

$$
P_{\text {net }}=P_{\text {ine }} \mathrm{CR}_{\mathrm{F}}-\mathrm{P}_{\text {ref }} \mathrm{CR}_{\mathrm{R}}
$$

where $P_{i n c}$ is the forward incident power, $P_{r e f}$ is the reverse reflected power measured on the sidearm of the coupler, and $\mathrm{CR}_{\mathrm{F}}$ and $\mathrm{CR}_{\mathrm{R}}$ are the forward and reverse coupling ratios for the coupler referenced to its output port.

The transmitting antennas used are open ended waveguides (200 to $500 \mathrm{MHz}$ ) and standard gain horns ( $500 \mathrm{MHz}$ to $18 \mathrm{GHz}$ ).

Comparisons of the response or susceptibility characteristics of EUT (or antennas) obtained using an anechoic chamber and a reverberation chamber are typically made in terms of peak values. The main reasons for this are that typically the EUT's worst case performance or susceptibility is desired, and the practical consideration of the difficulty in obtaining a true average response for an EUT from anechoic chamber data. Even determining the EUT's peak response in an anechoic chamber (depending of course upon how well behaved the EUT radiation pattern characteristic is) can require considerable measurements involving complete pattern measur ements.

\subsubsection{Measurement of the Peak Response of a $1 \mathrm{~cm}$ Dipole and a Ridged Horn}

A comparison of the peak output response data obtained for the $1.0 \mathrm{~cm}$ dipole using the NBS reverberation and anechoic chambers is given in figure 5.2. Note that the probe output response in the anechoic chamber is greater, in general, by about 2.5 dB than its output in 
the reverberation chamber. This suggests that the correlation between the results may be related to the EUT's gain characteristics in the two chambers (i.e., the gain of the dipole probe in free space is approximately $2.5 \mathrm{~dB}$ ). Determination of a far-field gain for a complex receptor (for example the EUT described in section 4.3.4) is extremely difficult. Therefore, a simple, well defined broadband receiving antenna (a ridged horn) was used as an EUT to repeat similar tests performed for the $1 \mathrm{~cm}$ dipole. The horn is designed to operate in the frequency range $800 \mathrm{MHz}-10 \mathrm{GHz}$. For these measurements in the anechoic chamber, the peak response (peak received power) of the horn occurs when the horn is bore-site aligned and polarization matched with the source antenna. These measurement results were then compared with the horn's peak received power in the reverberation chamber with the same level exposure field. The results are shown in figure 5.3a. Note that the horn's response is greater in the anechoic chamber. To see if this difference corresponded to the difference in the gain characteristics of the horn in the anechoic chamber, as compared to that in the reverberation chamber, the horn was calibrated to determine its far-field gain in the frequency range 800 $\mathrm{MHz}-10 \mathrm{GHz}$. These results are shown in figure 5.3b. Again, note the general agreement between the difference in the horn's response measured in the two facilities and the horn's far-field gain.

\subsubsection{Measurement of RF Coupling Through Apertures in TEM Lines}

Measurements were made using the reverberation and anechoic chambers to evaluate the $r f$ coupling through apertures cut into a series of TEM cells or transmission lines. Three TEM cells were used. Similar models (EUTs) have been used for evaluating shielding effectiveness of connector assemblies [23]. Electromagnetic energy coupled through the aperture in the TEM cell when exposed to the test field excites the fundamental TEM mode inside the cell. This results in $\mathrm{rf}$ power conducted to the cell's output ports which can then be measured. These results are compared with the theoretically predicted values based upon a planar field exposure to determine degree of correlation. The results obtained for one of the TEM cells, shown in the photograph of figure 5.4, are given in figure 5.5. The dimensions for this cell are $12 \mathrm{~cm} \times 18 \mathrm{~cm} \times 36 \mathrm{~cm}$ with a $5.1 \mathrm{~cm} \times 5.1 \mathrm{~cm}$ aperture centered in the top of the outer conductor. The theoretically predicted peak coupling for a uniform field exposure is also shown on figure 5.5 [24]. Measurements were not made in the anechoic chamber at frequencies below $500 \mathrm{MHz}$ because of the limitations of the anechoic chamber measurement system. The theoretical peak values should be essentially the same as the measured values in the anechoic chamber. Again, the anechoic chamber peak data are generally higher than the peak reverberation chamber data. Also, the average reverberation chamber data are approximately 7-8 $\mathrm{dB}$ lower than the peak data, similar to both the $1 \mathrm{~cm}$ dipole and ridged horn results.

Results obtained for the second cell, shown in figure 5.6, are given in figure 5.7. The dimensions for this cell are $12 \mathrm{~cm} \times 12 \mathrm{~cm} \times 24 \mathrm{~cm}$ with a $3.1 \mathrm{~cm}$ diameter aperture. The results are similar to those shown for the larger cell. Results obtained for the third cell, shown in figure 5.8, are given in figure 5.9. The dimensions for this cell are $3.0 \mathrm{~cm} x 6.0$ $\mathrm{cm} \times 11.4 \mathrm{~cm}$ with a $1.4 \mathrm{~cm}$ diameter aperture centered in the top of the outer conductor. Again the results are similar.

\subsubsection{Measurements of the EM Susceptibility of a Modified $7.0 \mathrm{~cm}$ Folding Fin Aircraft Rocket} (FFAR) [25].

A $7.0 \mathrm{~cm}$ diameter FFAR was modified with a thermocouple element to sense the response of the rocket's inserted electro-explosive device (EED). This was done to allow measurement of the $r f$ current coupling into the EED's bridge wire circuit when the rocket is exposed to an $r f$ field. The rocket was also modified with a $1.27 \mathrm{~cm}$ plastic spacer at the base of its fin (on the tail section) to increase the rf coupling to the bridge wire circuit. This lessened the requirements for high $r f$ power to generate fields sufficient to perform these measurements. This modification was justified, realizing the purpose of this study was to compare susceptibility results obtained for the rocket in different environments and not to simply evaluate an EUT's susceptibility to EMI. Photographs showing the rocket placement inside the NBS reverberation chamber and the NBS anechoic chamber are shown in figures 5.10 and 5.11 . Measurement results of the rocket's thermocouple peak output current resulting from rf coupling as a function of frequency are shown in figures $5.12 \mathrm{a}$ and $5.12 \mathrm{~b}$. These data were

obtained using exposure fields normalized to $10 \mathrm{~mW} / \mathrm{cm}^{2}$ in each of the chambers. The anechoic chamber data were obtained by rotating the rocket in azimuth in a planar far-field using both vertical and horizontal polarizations. The rocket was rolled a few degrees around its axis before each azimuth cut. Sufficient roll angles were used to determine the peak response. Examples of pattern data obtained in the anechoic chamber at three selected frequencies are shown in figure 5.13. A total of 719 patterns were obtained in the anechoic chamber from 
which the peak response at each frequency was determined. These data were then used to plot curve A of figure 5.12a. Curve A (anechoic chamber data) indicated greater response or more susceptibility, except at one frequency (1800 MHz), than curve B (reverberation chamber peak response). Again, the proposed explanation for this is the difference between the gain characteristics of the rocket's response in the anechoic chamber (for example see figure 5.13) and its gain characteristics in the reverberation chamber. The rocket gain is lost in the reverberation chamber. Figure $5.12 \mathrm{~b}$ shows the difference between curves $A$ and $B$ for $f i g u r e$ $5.12 a$.

\subsection{Comparison of Results Between Reverberation Chambers}

Measurements were made of the EM susceptibility response of three different EUTs, using two additional, similar, but different size reverberation chambers. These measurements were made to give an indication of the repeatability in measurement results obtained using the same technique (reverberation chamber methods) but with totally different facilities. The second and third reverberation chambers are located at the Naval Surface Weapons Center, Dahlgren, Virginia. These chambers were made from a single large shielded enclosure partitioned into two compartments with a removable center panel. The two chambers consist of: 1) the half chamber, $3.51 \mathrm{~m} \times 5.18 \mathrm{~m} \times 5.86 \mathrm{~m}$ in size using a little over half of the full enclosure, and 2) the full chamber, $3.51 \mathrm{~m} \times 5.18 \mathrm{~m} \times 10.82 \mathrm{~m}$ in size, using the full enclosure. The NSWC enclosure was constructed of continuously welded steel sheeting similar to the NBS enclosure. Two basic insights are provided by a comparison of results obtained from the evaluation of these different enclosures. First, that the input power requirements of a chamber is a function of its size and can be estimated based upon the calibration of a chamber of similar construction. Second, susceptibility test results obtained for the same EUT in different reverberation chambers are comparable. These conclusions are demonstrated in figures 5.14 to 5.17. Figure 5.14 shows a comparison of the fields inside the NBS and NSWC chambers calculated from the chamber's reference antenna received power measurements and then measured by a calibrated $1 \mathrm{~cm}$ dipole probe. The net input power was normalized to one watt for all three chambers. Note that the field inside the NBS chamber is approximately 4 dB stronger than the NSWC half chamber and $6 \mathrm{~dB}$ stronger than the NSWC full chamber. Recall that the power density inside a second chamber can be estimated from a calibrated chamber by using ( 9 ). The ratio of the average power densities for the NBS to NSWC full size chamber for the same net input power (see section 2.1) was 3.22. This is equal to approximately 5 dB, a little less than that indicated by comparing figures $5.14 \mathrm{a}$ and $5.14 \mathrm{c}$, but still within reason.

The second point of obtaining comparable susceptibility results using different chambers is demonstrated in figures 5.15 to 5.17. These graphs show the comparison in measuring the responses of the NBS $1 \mathrm{~cm}$ dipole probe and a rectangular single ridged horn to a normalized 37 $\mathrm{dB} V / \mathrm{m}$ field inside the three different chambers and the peak response of the $7.0 \mathrm{~cm} F F A R$ to a

normalized $10 \mathrm{~mW} / \mathrm{cm}^{2}$ power density inside the NBS and the NSWC half size reverberation chambers. In general, the agreement is within measurement tolerances except at $200 \mathrm{MHz}$ for the FFAR. This frequency approaches the lower limit for using the reverberation chambers and is also where the FFAR is most susceptible and hence where one would expect the greatest difference.

\section{Summary and Conclusions}

\subsection{Conclusions Drawn From Study}

1. The practical lower frequency limit for using the NBS enclosure as a reverberation chamber is approximately $200 \mathrm{MHz}$. This lower limit is due to a number of factors including insufficient mode density, limited tuner effectiveness, and ability to uniformly excite all modes in the chamber. These factors are a function of both chamber geometry and size. Increasing the inside dimensions of the chamber will lower the useful frequency limit in an approximately proportional manner.

2. Spatial variations in the E-field maximum and average values determined in the chamber's test volume are:

$\begin{array}{cc}\text { Frequency (MHz) Variation } & \text { in Measured E-Field } \\ & \\ 200 & < \pm 8 \mathrm{~dB} \\ 500 & < \pm 5 \mathrm{~dB} \\ 1000 & < \pm 3 \mathrm{~dB} \\ 2000 & < \pm 2 \mathrm{~dB}\end{array}$


These data were determined using the mode tuned approach with 200 tuner increments for one complete tuner revolution. The limitation for determining the spatial E-field variation is most likely due to the increasing mode density and hence field complexity in the chamber as a function of frequency and the insufficient sampling of the field resulting from the limited number of tuner positions used. The spatial E-field variations should decrease less than 2 dB at frequencies above $2 \mathrm{GHz}$ if sufficiently large data samples are taken.

3. Using a combination of antennas within their specified design frequency ranges for transmitting and as reference receiving antennas inside the chamber is preferred to the use of a long wire antenna. This is apparent because of the lower VSWR and greater efficiency in exciting the chamber and coupling energy from the chamber.

4. E-field data obtained using the mode stirred approach with the chamber empty have significantly less amplitude variations with frequency than data obtained using the mode tuned approach, particularly at frequencies above 1-2 GHz (e.g., <3 dB rather than <6 dB. Compare figure 2.26 to figure 3.3.) This implies that the mode stirring method is superior for some applications above these frequencies. This is significant since the stirred method is much simpler and faster to use. In comparing the two methods it is interesting to note the difference in the amplitude of the maximum fields at frequencies below 1-2 GHz. The mode tuned method is more accurate at these frequencies for determining the absolute amplitude of the test field since it allows for corrections due to changes in antenna VSWR. Because of these observations and other supporting data, the best results (accuracy versus measurement time considerations) for the NBS reverberation chamber can be obtained using the following approaches and tuner positions for susceptibility testing:

$\begin{array}{ccr}\text { Frequency Range } & \text { Method } & \text { \# Tuner Positions } \\ 0.2-1.0 \mathrm{GHz} & \text { Mode Tuned } & 200 \\ 1.0-2.0 \mathrm{GHz} & \text { Mode Tuned } & 400 \\ >2.0 \mathrm{GHz} & \text { Mode Stirred } & 5000\end{array}$

5. Lowering the $Q$ of the enclosure: a) increases the rf power required to obtain the test field, b) increases the uncertainty in determining the test field level, and c) decreases the spatial, statistical field uniformity.

6. The statistically determined average wave impedance of the EM field in the chamber is approximately that of free space (377 ohms), thus confirming the validity of (10).

7. The maximum E-field is approximately 7 to $8 \mathrm{~dB}$ greater than the average E-field.

8. Scattering from the EUT does not seem to influence the statistical, spatial E-field distribution within the chamber.

9. Multiple source excitation (from more than one location) improves the spatial E-field distribution slightly, but not enough to justify additional hardware requirements and the additional rf power needed to compensate for the loss in the additional input transmission line required.

10. Energy is conserved in the chamber (i.e., power coupled from the chamber via loss in transmission lines, antennas, EUT, physical support materials, etc., reduce the power density in the chamber. Thus it is good practice to remove all that are not essential to the tests from the chamber.

11. The chamber (NBS) continues to operate (as a reverberation chamber) with loading that reduces the Q significantly (up to 90\% of the total energy absorbed), however, with significant loss in measurement accuracy.

\subsection{Summary of Measurement Uncertainties}

\subsubsection{Determining the Field Strength Inside the Chamber}

Recall from section 2.3.1 that the susceptiblity/vulnerability test field established inside the chamber can be expressed either in terms of an "equivalent" power density (8) or an "equivalent" E-field strength (10). An estimate of the uncertainties in each of these quantities can be obtained by analyzing the contributing parameters involved in each 
mode-tuned and mode stirred approaches respectively, within their appropriate frequency bands. Five major categories are identified. The first is the uncertainty in determining the received power from the chamber's reference antenna. This is broken up into four components: namely, cable loss, attenuator calibration, reference antenna efficiency and power meter or spectrum analyzer measurements uncertainties. Values shown are typical of estimated uncertainties stated for these type of measurements and instruments.

The second category is the impedance mismatch error that can occur between the reference receiving antenna(s) (source) and the power detector(s) (load). The actual power delivered to the load is a function of the impedance match between the source and load, with maximum power transfer occurring when a conjugate impedance match exists.

Power transfer between a source and a load is given as

$$
\begin{aligned}
& \text { fraction of maximum }=\left(1-\left|\Gamma_{S}\right|^{2}\right)\left(1-\left|\Gamma_{L}\right|^{2}\right)
\end{aligned}
$$

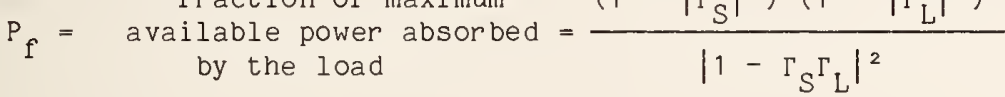

where $\Gamma_{S}$ and $\Gamma_{L}$ denote complex reflection coefficients. The magnitudes, $\left|\Gamma_{S}\right|$ and $\left|\Gamma_{L}\right|$ can be obtained from the appropriate VSWR by the expressions

$$
\left|\Gamma_{i}\right|=\frac{V S W R-1}{V S W R+1}, \quad i=S \text { or } L .
$$

The VSWRs for the reference antennas (sources) and power detectors (loads) used in the NBS reverberation chamber are given in table 6.3. These values were used to calculate the estimated uncertainties shown for the mismatch error in tables 6.1 and 6.2 . Note that different average and maximum values are given.

The third category of error, referred to as mixing or sampling efficiency, is divided into two parts. The first part relates to the ability to obtain a uniform spatial field distribution (statistically) inside the chamber and to effectively destroy the polarization characteristics of the exposure field. (i.e., the statistically determined response characteristics of the EUT and chamber reference antenna are independent of their directional properties.) The second part is the uncertainty due to limiting the number of tuner positions per revolution when performing the measurement. This source of uncertainty is different when determining the average as compared to the maximum field as shown in the tables. Data shown in figure 2.27 and table 6.4 were used in obtaining these estimates.

The fourth category of uncertainty relates only to determining the equivalent E-field strength in the chamber from the equivalent power density. Recall that (10) assumes that the equivalent wave impedance inside the chamber is $120 \pi$ ohms. In reality this is not true as was shown in figure 2.25. This figure was then used to provide an estimate for this error taking into account that a significant amount of data obtained to date indicates that peak fields are approximately 7-8 dB greater than the average field strength inside the chamber (see section 2.3.2). i.e., An examination of figure 2.25 shows values of wave impedance as 1 arge as 1600 ohms at frequencies below $500 \mathrm{MHz}$ when the maximum E-field was measured. This would require a large correction, up to $6.3 \mathrm{~dB}$. However, experience indicates that a well behaved relationship (7-8 dB difference) exists between the measured peak and average values of the Efield. This suggests that the peak value of the wave impedance for maximum E-field inside the chamber decreases as the frequency increases thus lowering this source of error. These observations are reflected in the uncertainty estimates shown in the tables.

The fifth source of error occurs if one fails to correct for net input power variations due to the loading effect of the chamber on the VSWR of the source antennas. (See section 4.2 for more details.) Note that these corrections are made typically when using the mode tuned approach and hence are not included in the total or root-sum-square error estimates of table 6.1. They are shown on the table to provide insight to the estimated magnitude of error expected if this correction were not made. The basis for these estimates are the data shown in figures $6.1 \mathrm{a}$ and $6.1 \mathrm{~b}$. These figures show the average and maximum E-field strength measured inside the NBS reverberation chamber using an array of seven NBS isotropic probes. A comparison is shown between results obtained before and after the measurements were corrected for net input power variations and normalized for an equivalent constant net input power. Note from table 6.1 this error is the same order of magnitude as 2), the mismatch error between the receiving antennas and power detectors. This is as expected since the source and 
receiving antennas used in the chamber have similar VSWRs and the power detector (load) and generator VSWRs are also similar. This error is included in table 6.2 and estimated to be the same as for the receiving mismatch error.

The total (worst-case) uncertainties for each approach (mode-tuned, and mode-stirred) are shown at the bottom of each table. This uncertainty should be regarded as a conservative estimate. The probability of the true value of $E_{a}$ being near the extreme of such worst-case uncertainty is small. This is because the probability of every error source being at its extreme value and in the worst possible combination is almost zero.

A more realistic method of combining uncertainties is the root-sum-of-the-squares ( $r s s$ ) method. The rss uncertainty is based on the fact that most of the errors are independent of each other and hence are random with respect to each other and combine like random variables. The rss method of combining random variables is justified by statistical considerations (beyond the scope of this report) which are also inherent in the reverberation chamber measurement methods.

Finding the rss uncertainty requires that each individual uncertainty be expressed in fractional form. The method of calculation follows the name - square the components, sum those squares and then take the square root. The results are shown at the bottom of tables 6.1 and 6.2 .

\subsubsection{General Comments on Error Analysis.}

Some general comments on interpreting immunity measurement results uncertainties based upon the above experimental error analysis are appropriate.

1) The mismatch error at frequencies below $2 \mathrm{GHz}$, (particularly if corrections are not made for either the transmitting antennas or the receiving antennas mismatch looking into their source or load), will cause the field determination inside the chamber to be low. This also causes the EUT response results to be lower than they actually are. For example, the low frequency data of figures 5.15, $1 \mathrm{~cm}$ dipole, and 5.16, ridged horn, should be corrected (response increased) proportionally to the systematic offset error estimates show in tables 6.1 and 6.2 . (i.e., $3.46 \mathrm{~dB}$ at $1.0 \mathrm{GHz}$ and $1.66 \mathrm{~dB}$ at $2.0 \mathrm{GHz}$ ).

2) The wave impedance, when the peak response of an EUT is measured, appears to be higher than $120 \pi$ ohms. This means that if the free space wave impedance of $120 \pi$ ohms is used in determining the corresponding peak amplitude of the exposure field, there will be a systematic offset error resulting in too low a calculated E-field exposure value, or, since the actual E-field is higher than the calculated value this results in too high a EUT response indication for a specified E-field exposure.

3) The spatial variation in the measured, statistically determined E-field in the chamber resulting from a complete revolution of the tuner decreases from as great as $\pm 8 \mathrm{~dB}$ at 200 $\mathrm{MHz}$ down to less than $\pm 2 \mathrm{~dB}$ at $2.0 \mathrm{GHz}$. (See figure 2.27.) Logically, it is expected that this variation will continue to decrease as the frequency increases. However, high variations exist in the response data obtained for EUT at frequencies where the spatial Efield variations are small. This is probably due to the relatively large variations in the wave impedance as a function of tuner position. Some additional influence may be due to the mismatch characteristics of the antennas vs source and load as a function of frequency. A way to reduce this problem is to increase the number of frequencies at which data are taken (clustered around a particular frequency of interest) or increase the number of reference receiving antennas or probes used to determine the exposure field (for example see figure 2.27) and then average the data.

\subsection{Measurement Technique Advantages and Limitations}

Significant advantages do exist, as alluded to in the introduction, for using a reverberation chamber for EMC measurements. Specific advantages and limitations for which insight was obtain from this study include:

a) The ability to generate high level fields efficiently. For example, 1 watt net input power into the NBS reverberation chamber results in electric fields of approximately 70 $\mathrm{V} / \mathrm{m}$. This is approximately $1 / 10$ the input power required to generate the same level field in the NBS anechoic chamber, assuming far-field separation distances. 
b) Large test zones, for example up to $2 / 3$ of the volume inside a reverberation chamber can be available excluding an area approximately $1 / 2$ meter spacing to the walls.

c) Broad frequency coverage (from $200 \mathrm{MHz}$ to at least $18 \mathrm{GHz}$ in the NBS chamber).

d) Testing is cost effective. This is especially true in comparison to anechoic chamber testing. Significant savings are realized in two major ways. First, the facility installation and measurement system procurement costs for a reverberation chamber are significantly less than for an anechoic chamber. Second, the time required to perform a complete EMC analysis of an EUT should be much less using a reverberation chamber. Again, from our experience in evaluating the susceptibility of the $7.0 \mathrm{~cm}$ FEAR, it required approximately $1 / 10$ the test time to obtain the reverberation chamber results as compared to the anechoic chamber results shown on $\mathrm{f}$ igure 5.13 .

e) The directional characteristics of antenna or EUT placed inside a reverberation chamber are lost resulting in an equivalent gain of unity.

f) The response of an antenna or EUT measured inside a reverberation chamber is less than when measured inside an anechoic chamber (open space) in proportion to its gain. Hence, it appears that the EUT's gain is the desired correlation factor. This implies that susceptibility criteria determined for an EUT using a reverberation chamber must include an additional factor proportional to the EUT's estimated maximum gain as a function of frequency.

g) The response of EUT to an electromagnetic field after it has penetrated the EUT's shield, appears to be equivalent in both the reverberation and anechoic chambers.

The advantages indicated above may well outweigh the disadvantages implied in items e and $f$, at least for some applications. The obvious trade off is one of measurement uncertainty that one can tolerate in determining the EMC/EMI characteristics of specific EUT and the inherent measurement uncertainties associated with determining the amplitude of the test fields inside the reverberation chamber.

\section{Suggestions for Future Research Efforts}

Because of the significant potential of the reverberation chamber method for performing immunity measurements, considerable interest has been expressed to further evaluate this technique with the intent of extending its range of applications. Specifically, the following suggestions are offered.

1) Evaluate the reverberation chamber measurement method for pulsed $r f$ (down to 1 us pulse duration) immunity testing, (i.e., experimentally investigate pulse dispersion characteristics of the chamber). Measurement studies are in progress at NBS under the sponsorship of the NSWC and RADC to complete this task.

2) Determine the feasibility of extending the use of the chamber from $18 \mathrm{GHz}$ to $40 \mathrm{GHz}$. A plan exists to undertake such an effort in late 1985 at NBS under sponsorship of RADC.

3) Evaluate reverberation chamber excited as a TEM transducer, (i.e., determine the feasibility of consolidating a TEM cell and reverberation chamber into a single facility for testing from $10 \mathrm{kHz}$ to $18 \mathrm{GHz}$ and beyond). This item is suggested because of the unique potential of developing a single, shielded facility that could be used for EMC/EMV testing over the complete frequency range 1 isted above. This task is also planned at NBS under RADC sponsorship.

4) Investigate the feasibility of using the reverberation chamber for multiple frequency immunity testing. This is of interest because such complex fields of multiple frequencies can exist in typical operational environments.

5) Investigate the use of the reverberation chamber technique for measuring shielding effectiveness of connectors (evaluate MIL STD 1344 measurement technique), shielding materials, and enclosures in comparison to other techniques. This suggestion is included with the objective of improving the state of art in shielding measurements and to determine if correlation or agreement exists between results obtained using the reverberation chamber method and other established techniques. 


\subsection{Acknowledgments}

The work described in this report was jointly funded by the Naval Surface Weapons Center (NSWC), Dahlgren, Virginia; Rome Air Development Center (RADC), Griffiss Air Force Base, Rome, New York; and the National Bureau of Standards, (NBS), Boulder, Colorado. The authors express appreciation especially to John Bean, NSWC; Charles Blank, RADC; and Mark T. Ma, NBS for their support, helpful comments and editorial review. Acknowledgement is also given to John L. Workman for his assistance in performing many of the measurements included in this study.

\subsection{References}

[1] Mendes, H.A. A new approach to electromagnetic field-strength measurements in shielded enclosures. in Wescon Tech. Papers; Los Angeles, CA. 1968 August.

[2] Cummings, J.R. Translational electromagnetic environment chamber, a new method for measuring radiated susceptibility and emissions. Proc. IEEE Int. Symp. on EMC; 1975; San Antonio, Texas.

[3] Corona, P; Latmiral, G; Paolini E; Piccioli L. Performance of a reverberating enclosure for power measurements in the microwave range. 2nd Symp. Tech. Exhibition on EMC; 1977; Montreux, Switzerland. 419-427.

[4] Bean, J.L.; Hall, R.A. Electromagnetic susceptibility measurements using a mode-stirred chamber. Proc. IEEE Int. Symp. on EMC; 1978; Atlanta, GA.

[5] Corona, P. Electromagnetic reverberating enclosures: behavior and applications. Alta. Frequ.: Vol. 49; 1980. 154-158.

[6] Corona, P.; Latmiral, G; and Paolini, E. Performance and analysis of a reverberating enclosure with variable geometry. IEEE. Trans. on EMC; EMC-22(1): 2-5; 1980 February.

[7] Crawford, M.L. Evaluation of a reverberating chamber facility for performing EM radiated field susceptibility measurements. Nat. Bur. Stand. (U.S.) NBSIR 81-1638; 1981 February.

[8] Liu, B.H.; Chang, D.C.; and Ma, M.T. Eigenmodes and the composite quality factor of a reverberating chamber. Nat. Bur. Stand. (U.S.) NBS Tech. Note 1066; 1983 August.

[9] Ramo, S.; and Whinnery, J.R. Fields and Waves in Modern Radio, 2nd ed. New York: John Wiley \& Son, Inc.; 1960. 418-428.

[10] Liu, B.H.; Chang, D.C.; and Ma, M.T. Design considerations of reverberating chambers for electromagnetic interference measurements. Proc. IEEE. Int Symp. on EMC; 1983 ; Washington, DC. 508-512.

[11] Ma, M.T.; Kanda, M.; Crawford, M.L.; and Larsen, E.B. A review of electromagnetic compatibility/interference measurement methodologies. Proc of IEEE. 388-411; 1985 March.

[12] Tai, C.T. On the definition of the effective aperture of antennas. IEEE Trans. Antennas and Propag. AP-9: 224-225; 1961 March.

[13] Greene, F.M. Development of Electric and Magnetic Near-Field Probes. Nat. Bur. Stand. (U.S.) NBS Tech. Note 658; 1975 January.

[14] Roe, J.M. An improved technological basis for radiated susceptibility and emissions specifications. Proc. IEEE. Int. Symp. on EMC; 1978; Atlanta, GA.

[15] Roe, J.M. Design of tuner for TEMEC, McDonnel Douglas Corp., St. Louis, MO., Private Communication.

[16] Bean, J.L. Design of tuner for NSWC reverberating chamber, Naval Surface Weapons Center, Dahlgren, VA., Private communication. 
[17] MIL-STD-1377 (Navy). Effectiveness of Cables, Connectors, and Weapons Enclosures Shielding and Filters in Precluding Hazards of Electromagnetic Radiation to Ordinance, Measurement of. 1971 August.

[18] Lentz, R.; and Anderson, H. Reverberating chambers for EMC measurements. Proc. IEEE. Symp. on EMC; 1979; San Diego, CA.

[19] Crawford, M.L. and Koepke, G.H. Operational considerations of a reverberation chamber for EMC immunity measurements - some experimental results. Proc. IEEE. Nat. Symp. on EMC; 1984; San Antonio, TX.

[20] Wilson, P.F. Peak versus average response characteristics in a reverberation chamber. National Bureau of Standards, Boulder, CO, Private communication.

[21] Bensema, W.D.; Reeve, G.R.; and Koepke, G.H. A multisensor automated EM field measurement system. Proc. IEEE IMTC/85 Conf; 1985; Tampa, FL.

[22] Bowman, R.R. Calibration techniques for electromagnetic hazard meters: 500 MHz to 20 GHz. Nat. Bur. Stand. (U.S.) NBSIR 75-805; 1976 April.

[23] Pressel, P.I. Mismatch: a major source of error in shielding effectiveness measurements. Proc. Seventeenth Annual Connector and Interconnection Technology Symp; 1984 September; Anaheim, CA.

[24] Wilson, P.F. Apertured coaxial probe. Paper in progress.

[25] Bean, J.L. Hero susceptibility of a 2.75 Inch FFAR: comparative results obtained from mode-stirred chambers, anechoic chamber, and ground plane test facilities, Proc. IEEE IMTC/85; 1985; Tampa, FL. 


\section{LIST OF FIGURES and TABLES}

Figure 2.1. Distinct frequencies of modes in NBS $3.05 \mathrm{~m}$ wide $\times 4.57 \mathrm{~m} 10 \mathrm{ng} \times 2.74 \mathrm{~m} \mathrm{high}$ shielded chamber below $200 \mathrm{MHz}$.

Figure 2.2 Total number of modes as a function of operating frequency for the NBS chamber.

Figure 2.3 Total number of modes as a function of operating frequency for a square based chamber whose volume is the same as that of NBS chamber.

Figure 2.4 Total number of modes as a function of operating frequency for a cubic chamber whose volume is the same as that of NBS chamber.

Figure 2.5 An illustration of mode degeneracy (NBS chamber).

Figure 2.6 An illustration of mode degeneracy (square-based chamber).

Figure 2.7 An illustration of mode degeneracy (cubic chamber).

Figure 2.8 Cumulative distribution curve of the normalized $1 / \mathrm{Q}$ values in the $180 \mathrm{MHz}$ to 200 $\mathrm{MHz}$ frequency band for the NBS chamber.

Figure 2.9 Cumulative distribution curve of the normalized 1/Q values in the $330 \mathrm{MHz}$ to 350 $\mathrm{MHz}$ frequency band for the NBS chamber.

Figure 2.10 Cumulative distribution curve of the normalized $1 / \mathrm{Q}$ values in the $480 \mathrm{MHz}$ to 500 $\mathrm{MHz}$ frequency band for the NBS chamber.

Figure 2.11 Photograph of interior of NBS reverberation chamber.

Figure 2.12 Average and minimum losses between transmitted and received powers measured at antennas' terminals inside NBS reverberation chamber. Transmitting antennas are: log periodic $(200 \mathrm{MHz}-1000 \mathrm{MHz})$, ridged horn (1.0 GHz - $4.0 \mathrm{GHz})$, double ridged circular horn $(4.0 \mathrm{GHz}-18 \mathrm{GHz})$. Receiving antennas are: long wire (200 $\mathrm{MHz}-1000 \mathrm{MHz})$, ridged horn $(1.0 \mathrm{GHz}-4.0 \mathrm{GHz})$, double ridged circular horn $(4.0 \mathrm{GHz}-18 \mathrm{GHz})$.

Figure 2.13 Theoretical composite $\tilde{Q}$ and experimental $Q^{\prime}$ determined for NBS reverberation chamber. a) Theoretical and experimental values of $Q$ as a function of frequency. b) Ratio of the theoretical composite $\tilde{Q}$ to experimental $Q^{\prime}$ as a function of frequency.

Figure 2.14 Bulkhead panel used for accessing NBS reverberation chamber.

Figure 2.15 Tuner designs for use in reverberation chambers. a) McDonnell Douglas Corp. TEMEC tuner, b) NSWC chamber tuner, c) NBS chamber tuner.

Figure 2.16 Photograph of tuner, log-periodic transmitting antenna and NBS isotropic probes inside NBS reverberation chamber.

Figure 2.17 Details of tuner and stepping motor mounting for the NBS reverberation chamber.

Figure 2.18 Ratio of maximum to minimum received power obtained by rotating tuner in the frequency range $200 \mathrm{MHz}$ to $18 \mathrm{GHz}$. a) Empty chamber, b) Four pieces of $66 \mathrm{~cm} \mathrm{rf}$ absorber placed upright in center of chamber, $0.5 \mathrm{~m}$ above floor.

Figure 2.19 Cross sectional views of NBS reverberation chamber showing placement of tuner, transmitting and receiving antennas, and NBS probes.

Figure 2.20 Design of taper section of long wire antenna for use in Reverberation chamber.

Figure 2.21 Photograph showing long wire antenna and NBS probes placement inside NBS reverberation chamber. 
Figure 2.22 VSWR of transmitting antennas placed inside NBS reverberation chamber. a) Long wire antenna, chamber empty, b) Composite of three antennas, (log-periodic, 0.2$1.0 \mathrm{GHz}$ ), ( $r$ idged horn, $1.0-4.0 \mathrm{GHz}$ ), and (double ridged circular horn, 4.0-18 $\mathrm{GHz}$ ), chamber empty, and c) Composite of three antennas with chamber loaded with 4 pieces of $66 \mathrm{~cm}$ rf absorber placed upright in center of chamber, $0.5 \mathrm{~m}$ above floor.

Figure 2.23 Average and minimum losses between transmitted and received powers measured at antennas's terminals inside NBS reverberation chamber. Long wire antenna transmitting $(200 \mathrm{MHz}-18 \mathrm{GHz}$ ), composite of three antennasreceiving (log periodic $200 \mathrm{MHz}-1000 \mathrm{MHz}$, ridged horn, $1.0 \mathrm{GHz}-4.0 \mathrm{GHz}$, double ridged circular horn, 4.0 GHz - $18 \mathrm{GHz}$ ).

Figure 2.24 Average and minimum losses between transmitted and received powers measured at antennas' terminals inside NBS chamber with four pieces of $66 \mathrm{~cm}$ rf absorber upright in center of chamber, $0.5 \mathrm{~m}$ above floor. Composite of three antennas used for transmitting and receiving.

Figure 2.25 Magnitude of wave impedance inside NBS reverberation chamber.

Figure 2.26 Average and maximum E-field strengths inside empty NBS reverberation chamber for $1 \mathrm{~W}$ net input power determined from: a) composite of 3 antennas received power measurements, and b) calibrated $1 \mathrm{~cm}$ dipole probe measurements.

Figure 2.27 E-field strength measured inside NBS reverberation chamber using array of 7 NBS isotropic probes: (a) maximum, and (b) average. Net power normalized to 1 Watt. Transmitting antennas are: $l o g$ periodic $(100 \mathrm{MHz}-1 \mathrm{GHz})$, ridged horn $(1 \mathrm{GHz}-2$ $\mathrm{GHz}$ ).

Figure 2.28 Mean values of the E-field strength measured inside NBS reverberation chamber using array of 7 NBS isotropic probes with $1 \mathrm{~W}$ net input power: (a) average, and (b) maximum. Data determined from maximum and average values of the 7 probes ( 7 locations) for the three orthogonal components and their sum (total) using 200 tuner positions ( 1.8 degree increments).

Figure 3.1 Placement of metallic objects inside NBS reverberation chamber to evaluate scattering effects upon E-field spatial distribution. a) Photograph showing 30 $\mathrm{cm} \times 50 \mathrm{~cm} \times 60 \mathrm{~cm}$ welded aluminum box inside NBS reverberation chamber. b) Photograph showing rolling equipment rack inside NBS reverberation chamber.

Figure 3.2 Comparison of the mean value of the average and maximum E-field strength measured using array of probes placed inside NBS reverberation chamber with chamber empty or with scattering object placed at center of test zone. a) $30 \mathrm{~cm} \times 50 \mathrm{~cm} \times 60$ cm aluminum box, b) Rolling equipment rack.

Figure 3.3 Maximum E-field strength inside empty chamber and chamber loaded with 4 pieces of $66 \mathrm{~cm} \mathrm{rf}$ absorber for $1 \mathrm{~W}$ net input power determined from composite of 3 antennas received power measurements. Data obtained using mode stirring approach.

Figure 4.1 Block diagram of NBS reverberation chamber evaluation and EMC measurement system.

Figure 4.2 Major software tasks for performing and evaluation susceptibility tests using a reverberation chamber.

Figure 4.3 Flow diagram for mode-tuned measurements.

Figure 4.4 Flow diagram for mode-stirred measurements.

Figure 5.1 Block diagram of NBS anechoic chamber EMC/EMI measurement system. Side view of chamber. The outside width is $6.7 \mathrm{~m}$.

Figure 5.2 Comparison of $1 \mathrm{~cm}$ dipole probe's peak responses to EM field established inside NBS reverberation and anechoic chambers. Output normalized to E-field exposure of $37 \mathrm{~dB} \mathrm{~V} / \mathrm{m}$. 
Figure 5.3 Comparison of ridged horn's peak responses to EM field established inside NBS reverberation and anechoic chambers. Output from horn normalized to exposure Efield of $37 \mathrm{~dB} \mathrm{V/m}$. a) Antenna output versus frequency, b) Difference in output responses of ridged horn measured in NBS reverberation and anechoic chambers compared to calibrated gain of horn.

Figure 5.4 Photograph of $12 \mathrm{~cm} \times 18 \mathrm{~cm} \times 36 \mathrm{~cm}$ rectangular TEM cell with $5.1 \mathrm{~cm} 5.1 \mathrm{~cm}$ aperture.

Figure 5.5 Comparison of power coupled to one port of $12 \mathrm{~cm} \times 18 \mathrm{~cm} \times 36 \mathrm{~cm}$ TEM cell with $5.1 \mathrm{~cm} \times 5.1 \mathrm{~cm}$ aperture placed inside NBS reverberation and anechoic chambers.

Figure 5.6 Photograph of $12 \mathrm{~cm} \times 12 \mathrm{~cm} \times 24 \mathrm{~cm}$ rectangular TEM cell with $3.1 \mathrm{~cm}$ diameter aperture.

Figure 5.7 Comparison of power coupled to one port of $12 \mathrm{~cm} \times 12 \mathrm{~cm} \times 24 \mathrm{~cm}$ TEM cell with $3.1 \mathrm{~cm}$ diameter aperture placed inside NBS reverberation and anechoic chambers.

Figure 5.8 Photograph of $3.0 \mathrm{~cm} \times 6.0 \mathrm{~cm} \times 11.4 \mathrm{~cm}$ rectangular TEM cell with $1.4 \mathrm{~cm}$ diameter aperture.

Figure 5.9 Comparison of power coupled to one port of $3.0 \mathrm{~cm} \times 6.0 \mathrm{~cm} \times 11.4 \mathrm{~cm}$ TEM cell with $1.4 \mathrm{~cm}$ diameter aperture placed inside NBS reverberation and anechoic chambers.

Figure 5.10 Photograph of modified $7.0 \mathrm{~cm}$ Folding Fin Aircraft Rocket inside NBS reverberation chamber.

Figure 5.11 Photograph of modified $7.0 \mathrm{~cm}$ Folding Fin Aircraft Rocket inside NBS anechoic chamber.

Figure 5.12 Comparison of $7.0 \mathrm{~cm}$ modified FFAR thermocouple responses to EM field established inside NBS reverberation and anechoic chambers. Data normalized to exposure power density of $10 \mathrm{~mW} / \mathrm{cm}^{2}$. a) Thermocouple output vs frequency. b) Difference in thermocouple output measured in NBS reverberation and anechoic chambers.

Figure 5.13 Examples of azimuth patterns of $7.0 \mathrm{~cm}$ modified FFAR thermocouple response taken in NBS anechoic chamber.

Figure 5.14 Comparison of the average and maximum E-field strengths inside the NBS and NSWC reverberation chambers determined for $1 \mathrm{~W}$ net input power using 1 ) reference antenna received power measurements, and 2) calibrated $1 \mathrm{~cm}$ dipole probe measurements. (a) NBS chamber, (b) NSWC half chamber, and (c) NSWC full chamber.

Figure 5.15 Comparison of $1 \mathrm{~cm}$ dipole probe's peak responses to normalized E-field of $37 \mathrm{~dB}$ $\mathrm{V} / \mathrm{m}$ using NBS and NSWC reverberation chambers.

Figure 5.16 Comparison of ridged horn's peak responses to normalized exposure E-field of 37 $\mathrm{dB} V / \mathrm{m}$ using NBS and NSWC reverberation chambers.

Figure 5.17 Comparison of $7.0 \mathrm{~cm}$ modified FFAR thermocouple responses to EM field established inside NBS and NSWC half reverberation chambers. Data normalized to exposure power density of $10 \mathrm{~mW} / \mathrm{cm}^{2}$. a) Thermocouple output vs frequency. b) Difference in thermocouple output measured in NBS and NSWC reverberation chambers.

Figure 6.1 E-field strength measured inside NBS reverberation chamber using array of 7 NBS isotropic probes: (a) average, and (b) maximum. Comparison shown between results obtained before and after normalization of measurements to correct for changes in net input power. Normalized data corrected for net input power of 1.0 watt. chamber excitation antenna is $l o g$ periodic. $E_{p}$ is average field strength determined from 7 probes. 
Table 6.1 Summary and estimates of measurement uncertainties for determining field strength inside NBS reverberation chamber - Mode Tuned (200 MHz - 2.0 GHz).

Table 6.2 Summary and estimates of measurement uncertainties for determining field strength inside NBS reverberation chamber - Mode Stirred $(2.0 \mathrm{GHz}-18.0 \mathrm{GHz})$.

Table 6.3 Estimates of impedance mismatch uncertainties for received power measurements.

Table 6.4 Estimates of uncertainties due to limiting number of tuner positions (sample size). 

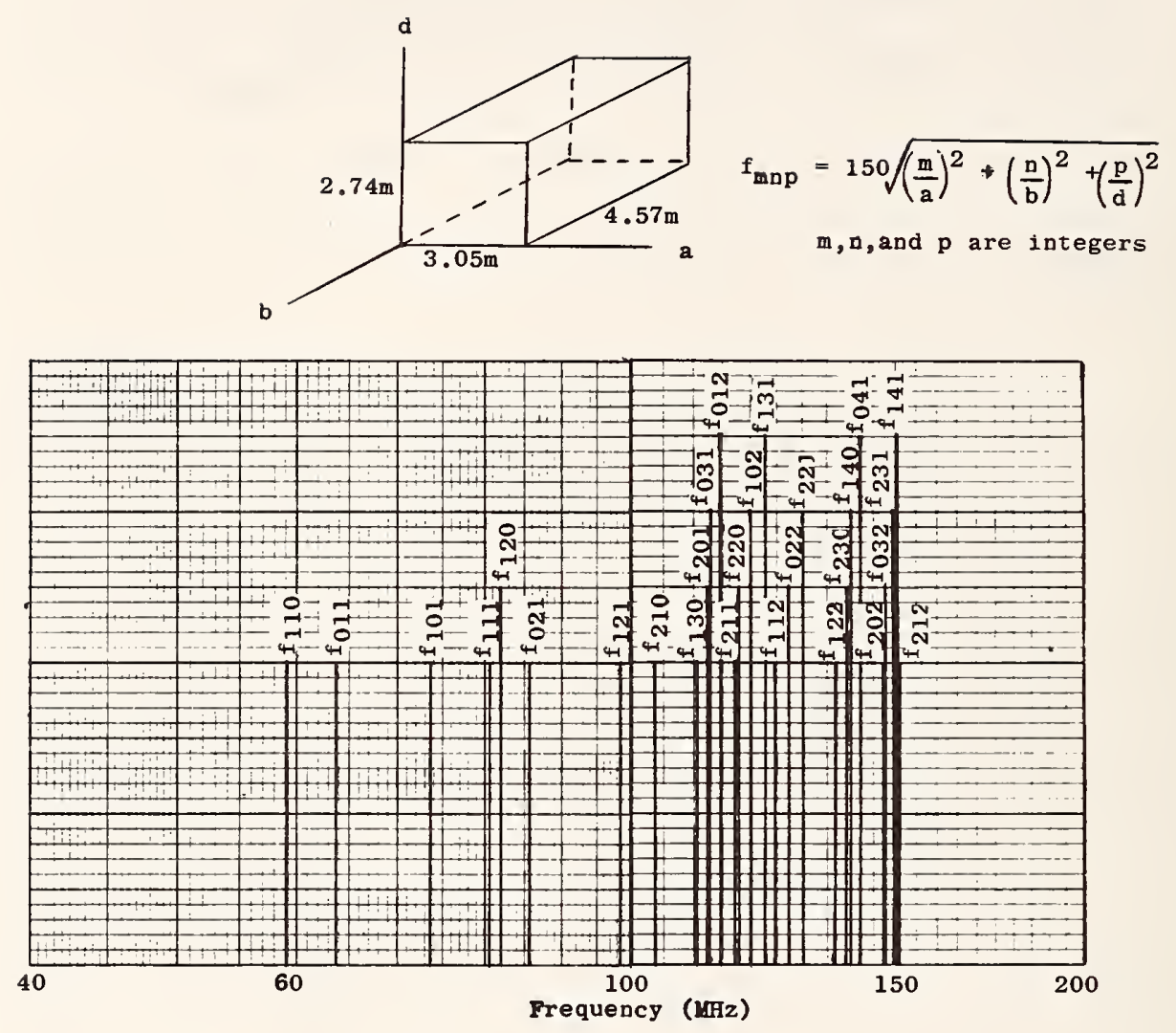

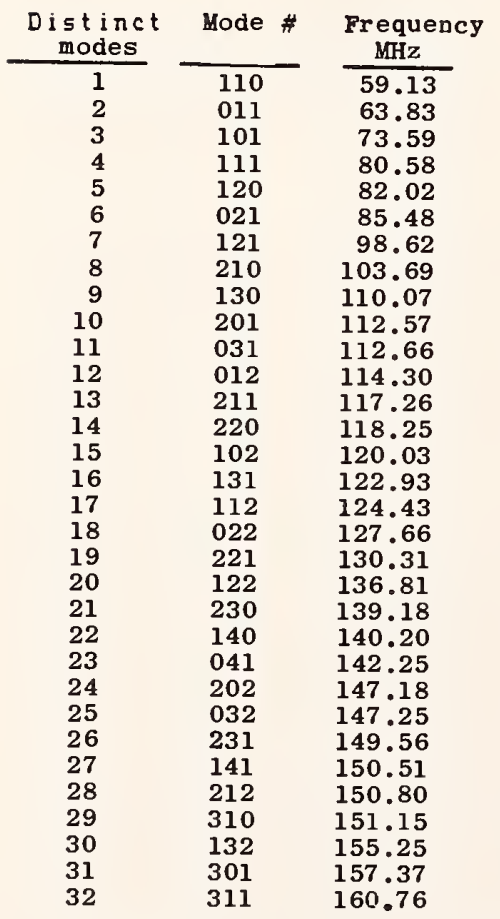

\begin{tabular}{|c|c|c|}
\hline $\begin{array}{c}\text { Dist inct } \\
\text { modes } \\
\end{array}$ & $\begin{array}{l}\text { Mode \# } \\
f_{\text {mnp }}\end{array}$ & $\begin{array}{l}\text { Frequency } \\
\quad \mathrm{MHz} \\
\end{array}$ \\
\hline 33 & 222 & $\overline{161.16}$ \\
\hline 34 & 320 & 161.49 \\
\hline 35 & 240 & 164.05 \\
\hline 36 & 013 & 167.48 \\
\hline 37 & 321 & 170.51 \\
\hline 38 & 042 & 170.95 \\
\hline 39 & 150 & 171.32 \\
\hline 40 & 103 & 171.44 \\
\hline 41 & 241 & 172.94 \\
\hline 42 & 051 & 173.00 \\
\hline 43 & 113 & 174.55 \\
\hline 44 & 023 & 176.87 \\
\hline 45 & 232 & 177.08 \\
\hline 46 & 330 & 177.38 \\
\hline 47 & 142 & 177.89 \\
\hline 48 & 151 & 179.86 \\
\hline 49 & 123 & 183.58 \\
\hline 50 & 302 & 183.73 \\
\hline 51 & 331 & 185.64 \\
\hline 52 & 312 & 186.64 \\
\hline 53 & 250 & 191.33 \\
\hline 54 & 203 & 191.44 \\
\hline 55 & 033 & 191.48 \\
\hline 56 & 213 & 194.23 \\
\hline 57 & 322 & 195.10 \\
\hline 58 & 242 & 197.23 \\
\hline 59 & 052 & 197.28 \\
\hline 60 & 340 & 197.50 \\
\hline 61 & 133 & 197.71 \\
\hline 62 & 251 & 199.01 \\
\hline 63 & 410 & 199.44 \\
\hline 64 & 223 & 202.38 \\
\hline
\end{tabular}

Figure 2.1 Distinct frequencies of modes in NBS $3.05 \mathrm{~m}$ wide by $4.57 \mathrm{~m}$ long $\times 2.72 \mathrm{~m}$ high shielded chamber below $200 \mathrm{MHz}$. 


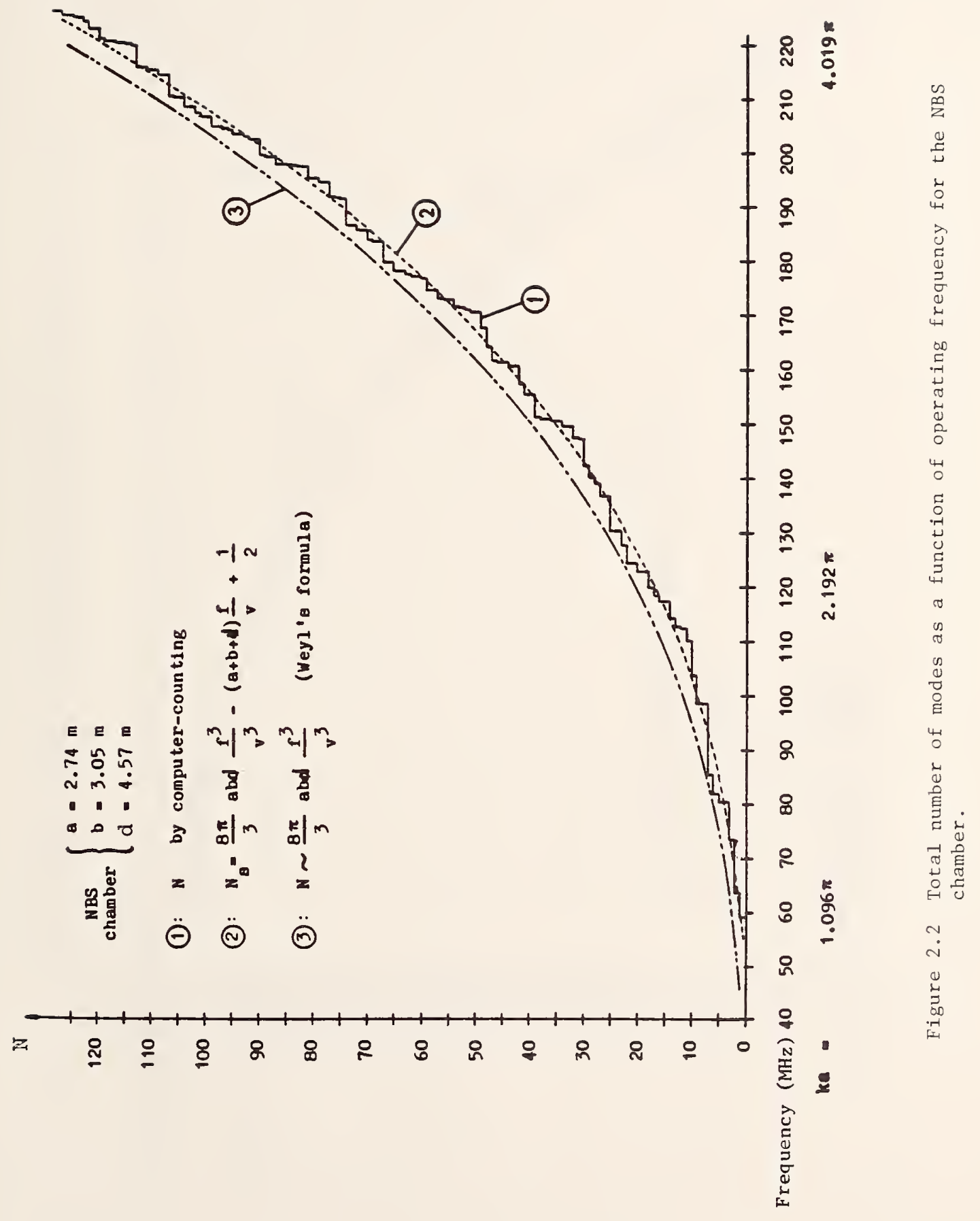




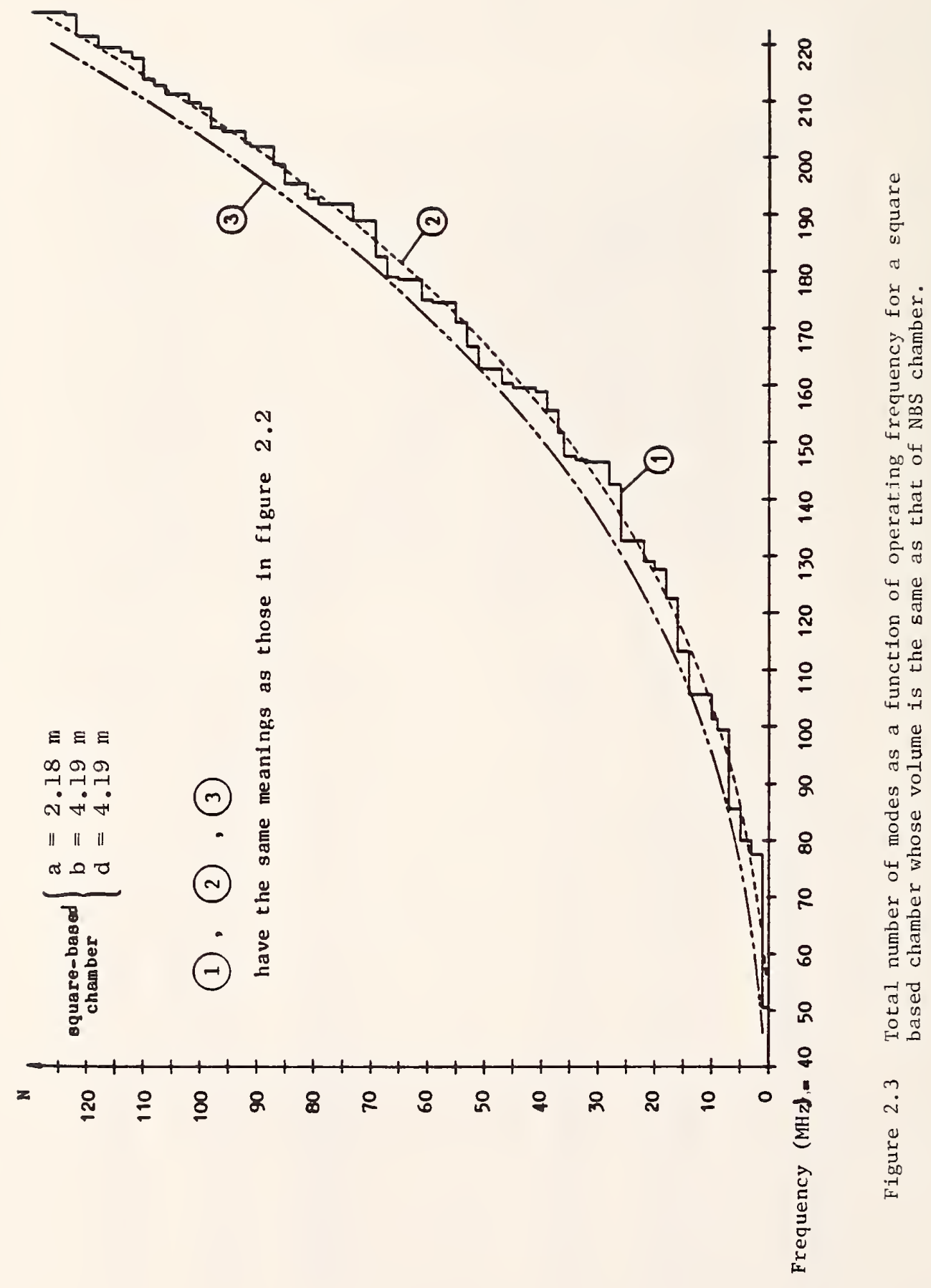




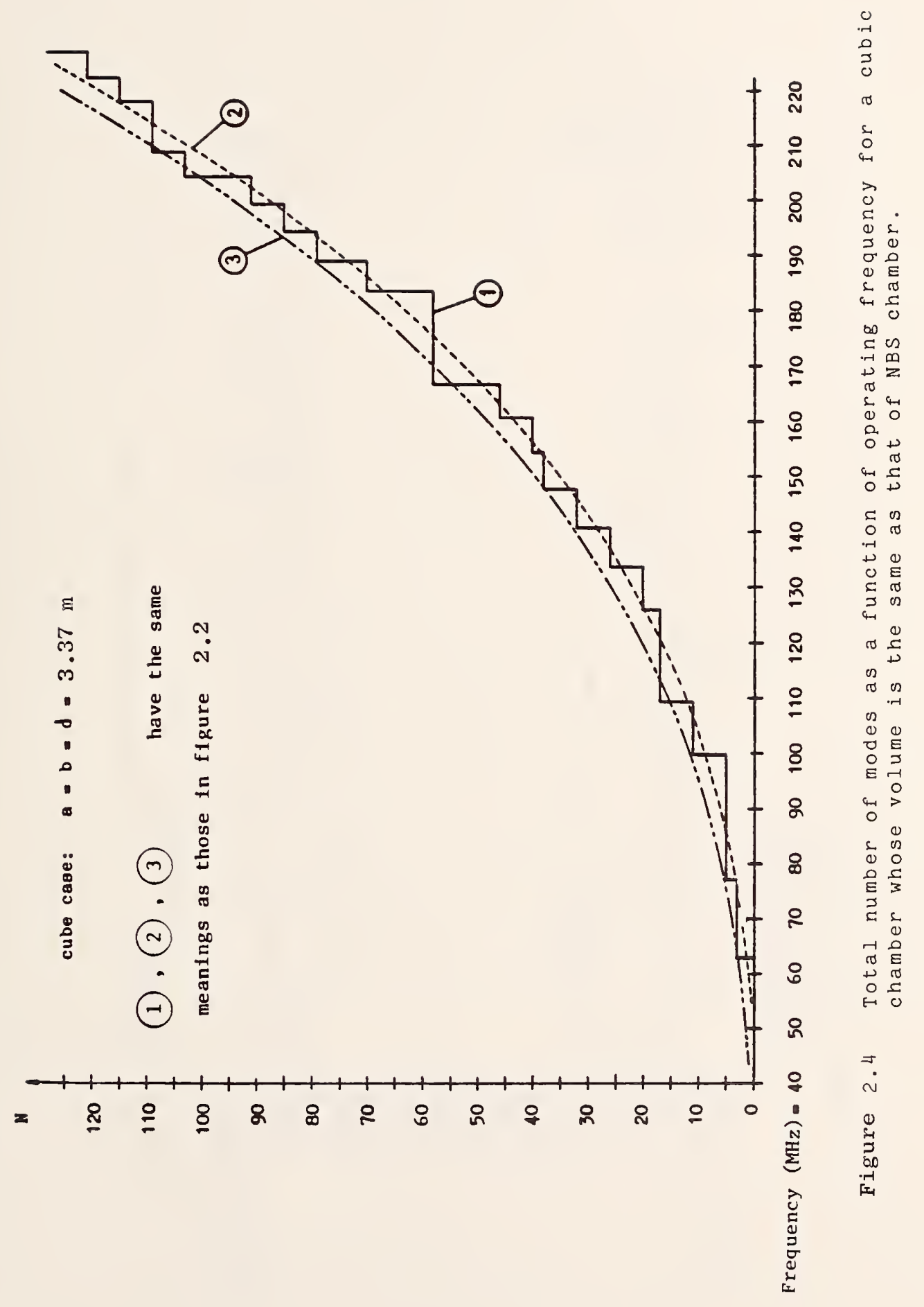




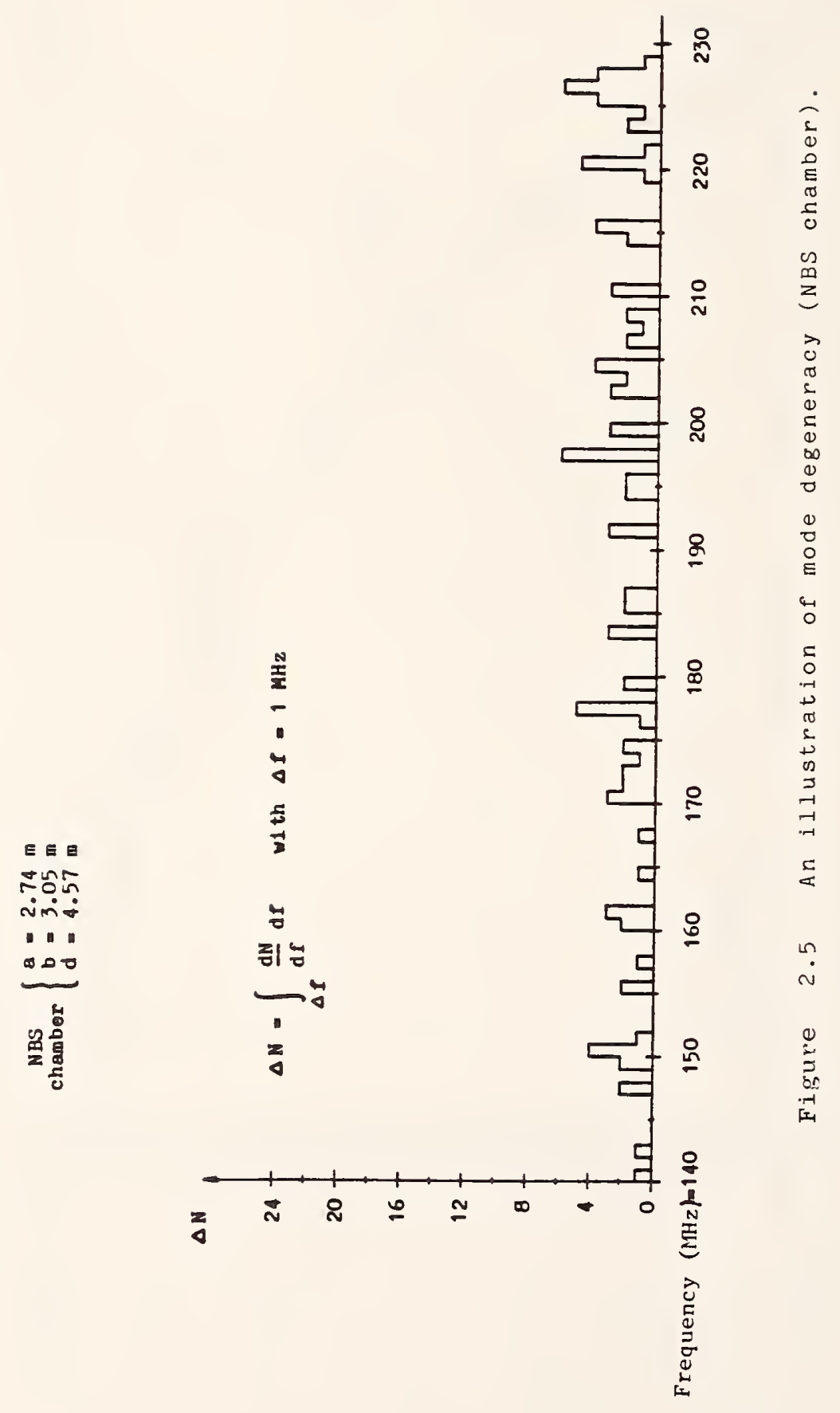




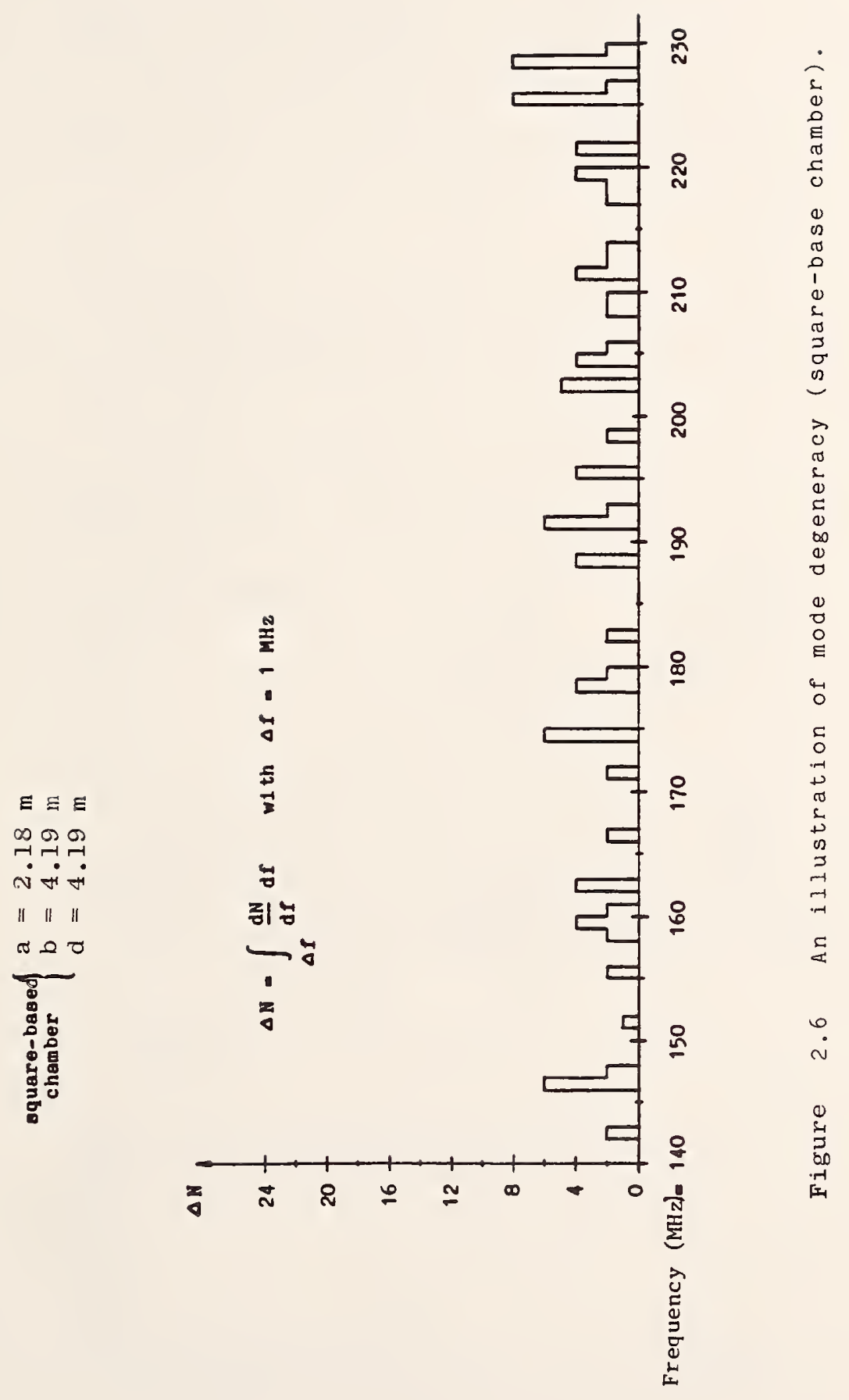




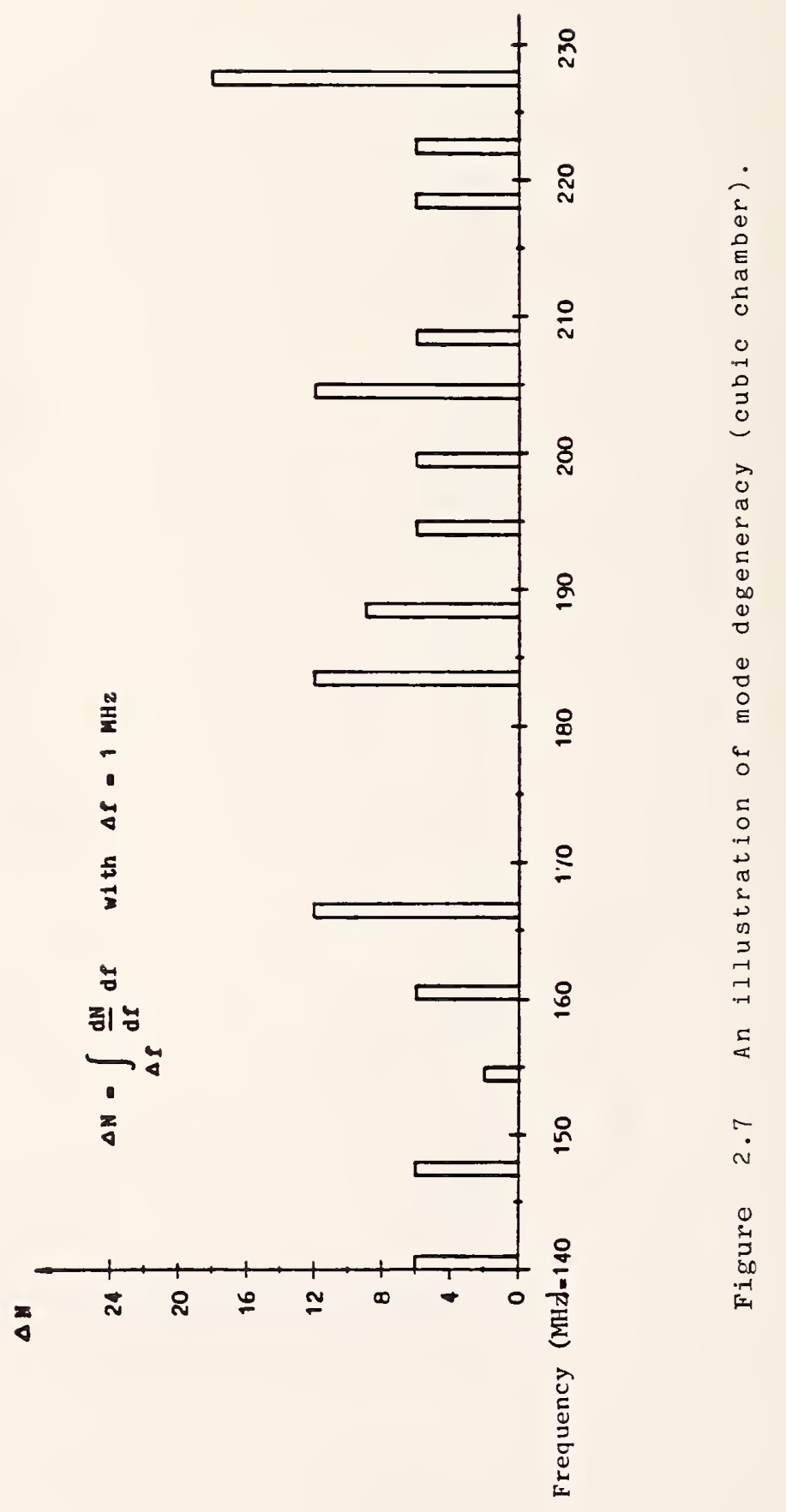




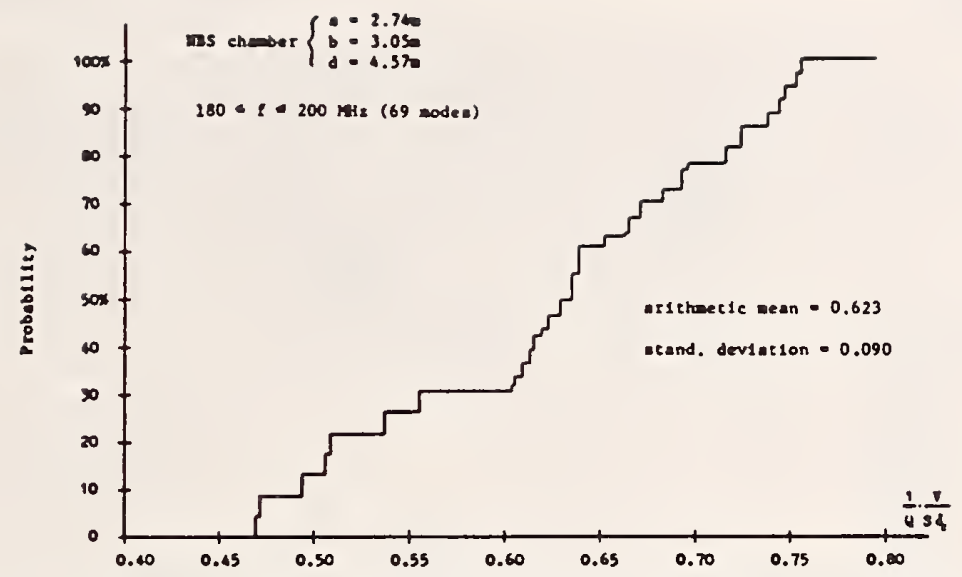

Figure 2.8 Cumulative distribution curve of the normalized $1 / 2$ values in the $180 \mathrm{MHz}$ to $200 \mathrm{MHz}$ frequency band for the NBS chamber.

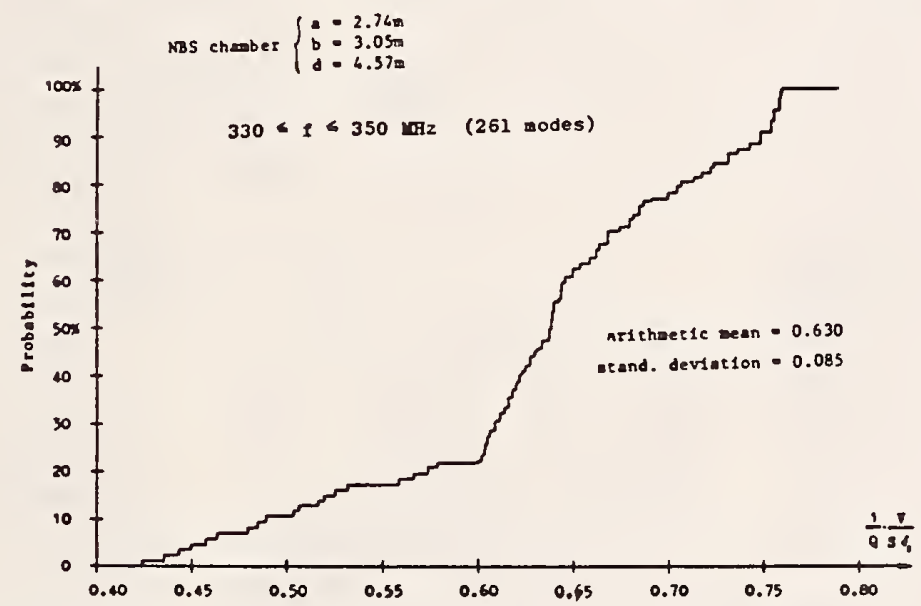

Figure 2.9 Cumulative distribution curve of the normalized $1 / 2$ values in the $330 \mathrm{MHz}$ to $350 \mathrm{MHz}$ frequency band for the NBS chamber.

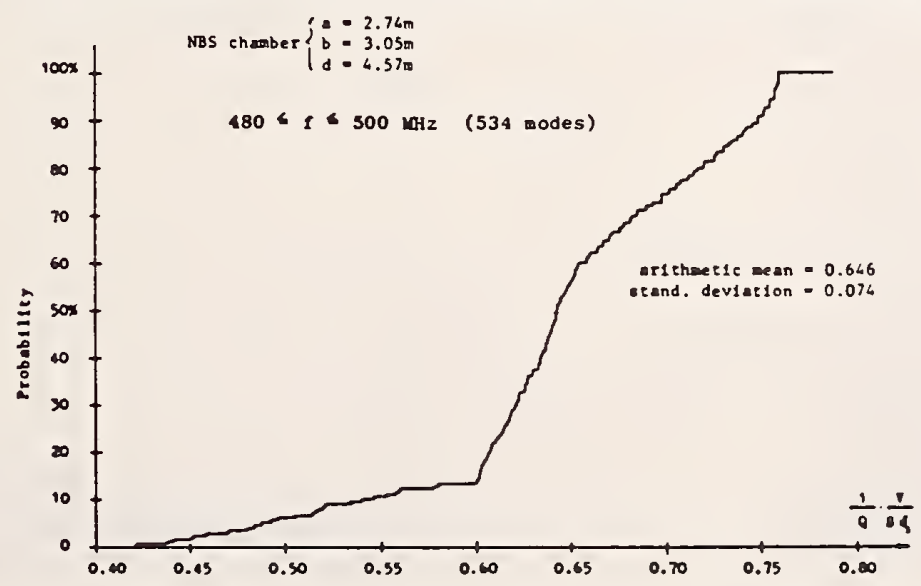

Figure 2.10 Cumulative distribution curve of the normalized $1 / Q$ values in the $480 \mathrm{MHz}$ to $500 \mathrm{MHz}$ frequency band for the NBS chamber. 


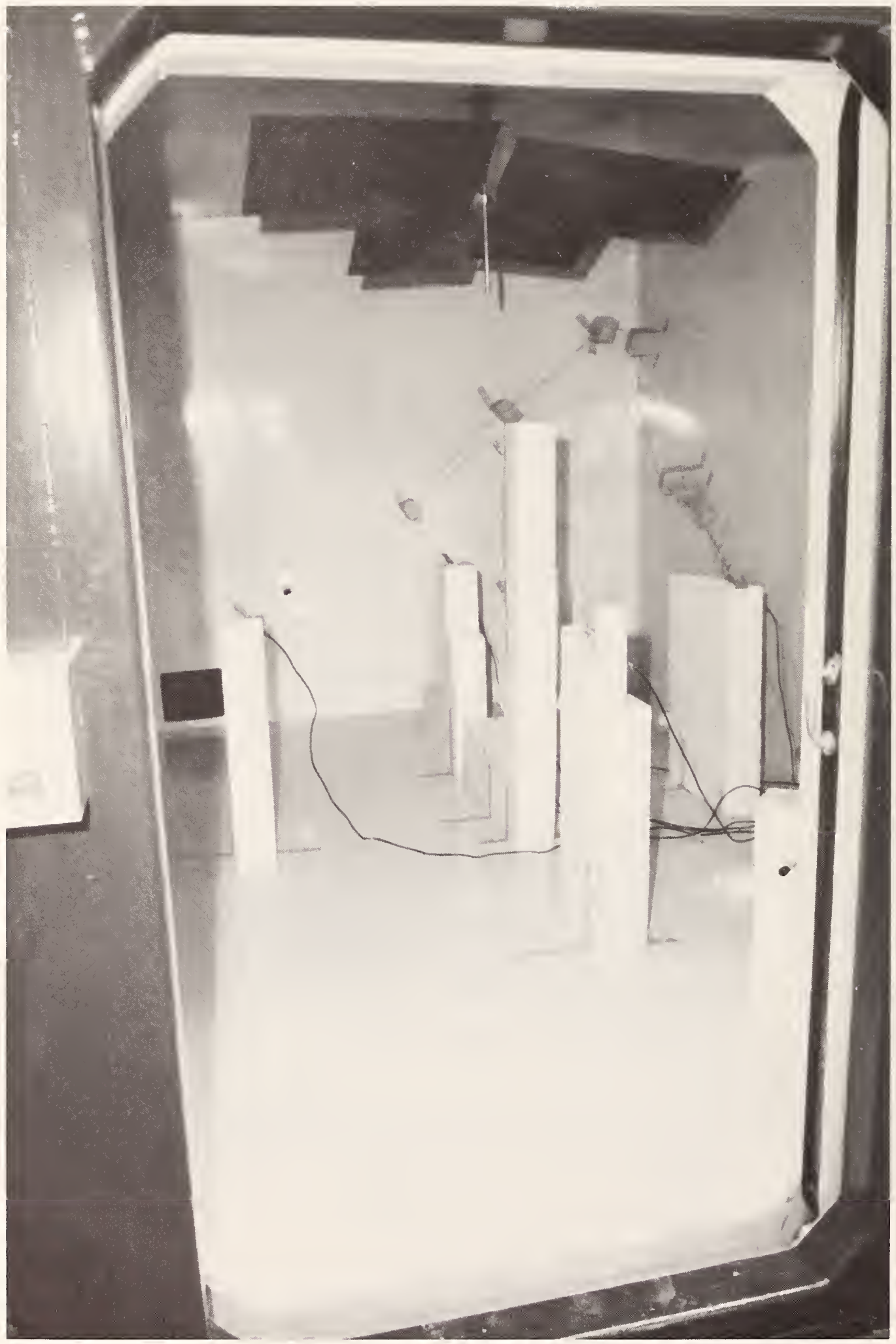

Figure 2.11 Photograph of interior of NBS reverberation chamber. 


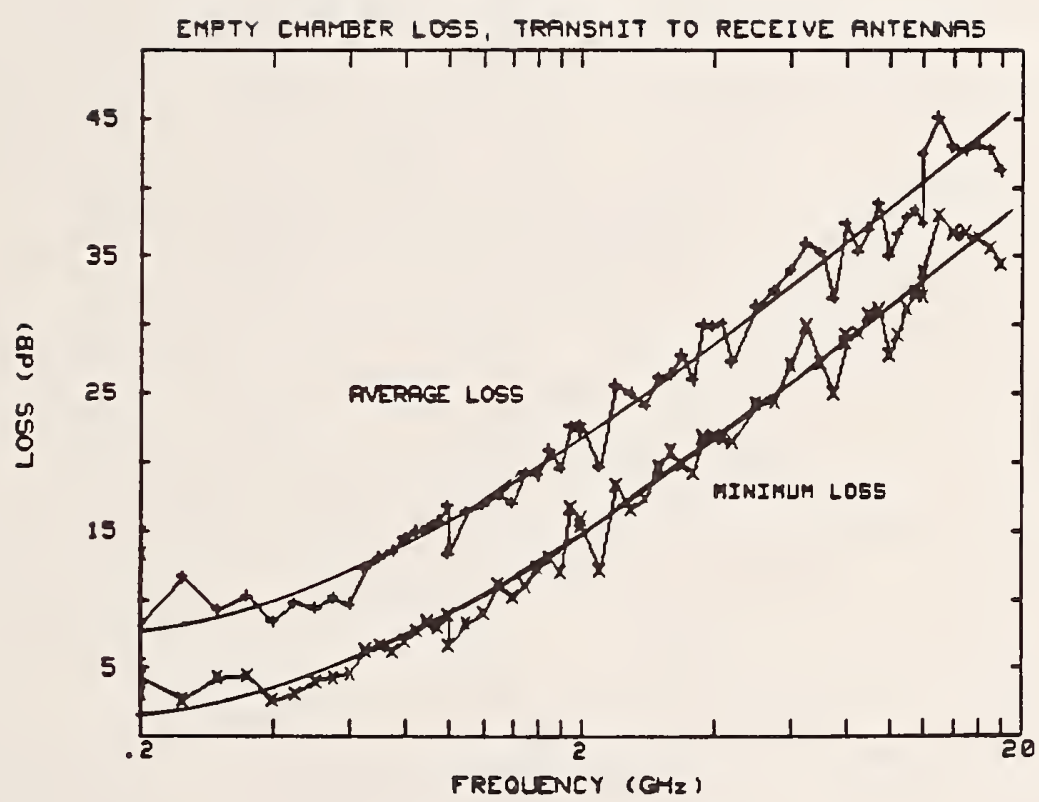

Figure 2.12 Average and minimum losses between transmitted and received powers measured at antennas' terminals inside NBS reverberation chamber. Transmitting antennas are: $10 \mathrm{~g}$ periodic (200 MHz $1000 \mathrm{MHz})$, ridged horn $(1.0 \mathrm{GHz}-4.0 \mathrm{GHz})$, double ridged circular horn $(4.0 \mathrm{GHz}-18 \mathrm{GHz})$. Recelving antennas are: long wire (200 $\mathrm{MHz}-1000 \mathrm{MHz})$, ridged horn $(1.0 \mathrm{GHz}-4.0 \mathrm{GHz})$. double ridged circular horn $(4.0 \mathrm{GHz}-18 \mathrm{GHz})$. 

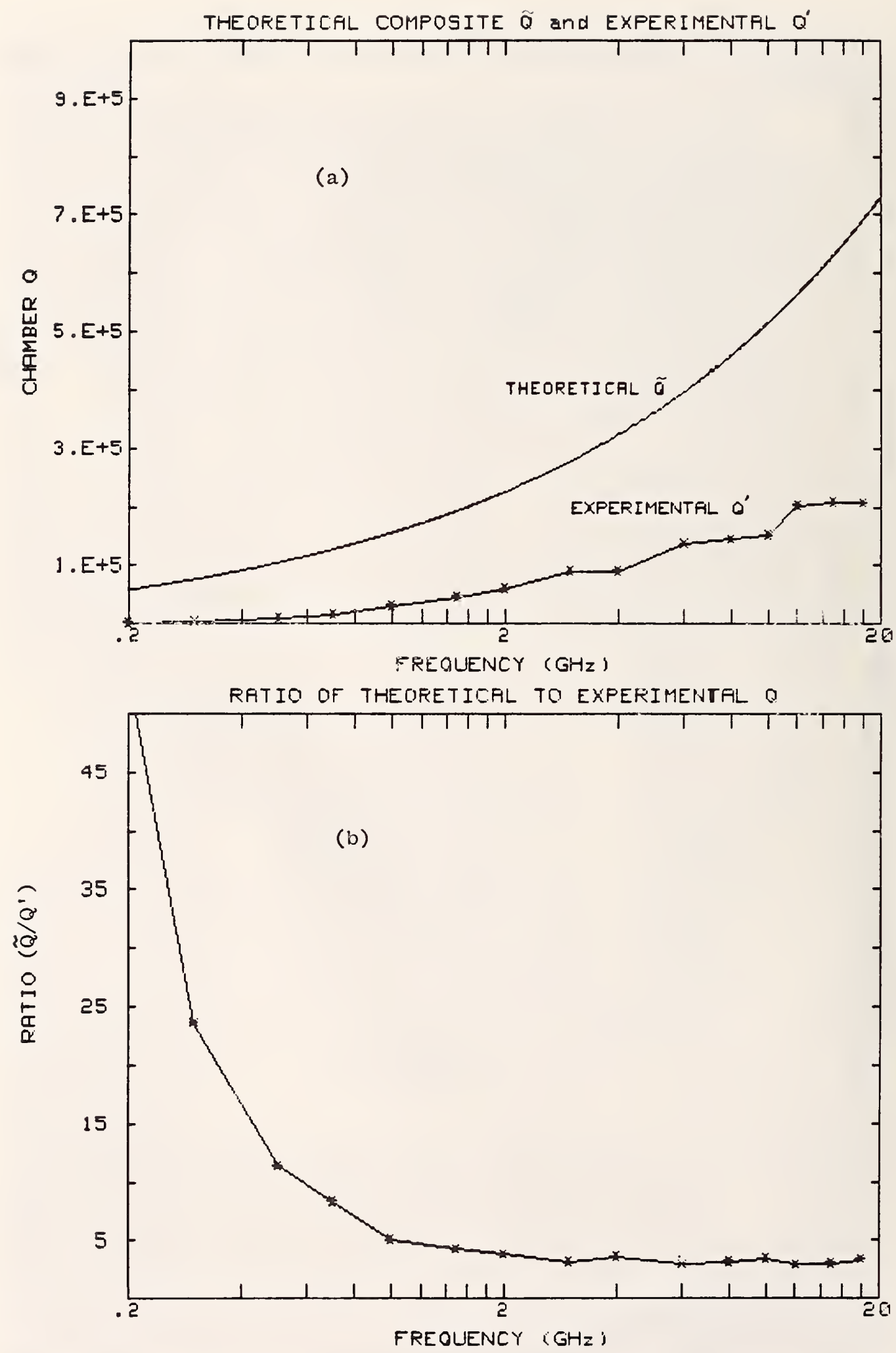

Figure 2.13 Theoretical composite $\tilde{Q}$ and experimental $Q^{\prime}$ determined for NBS reverberation chamber. a) Theoretical and experimental values of $Q$ as a function of frequency. b) Ratio of the theoretical composite $\tilde{Q}$ to experimental $Q^{\prime}$ as a function of frequency. 


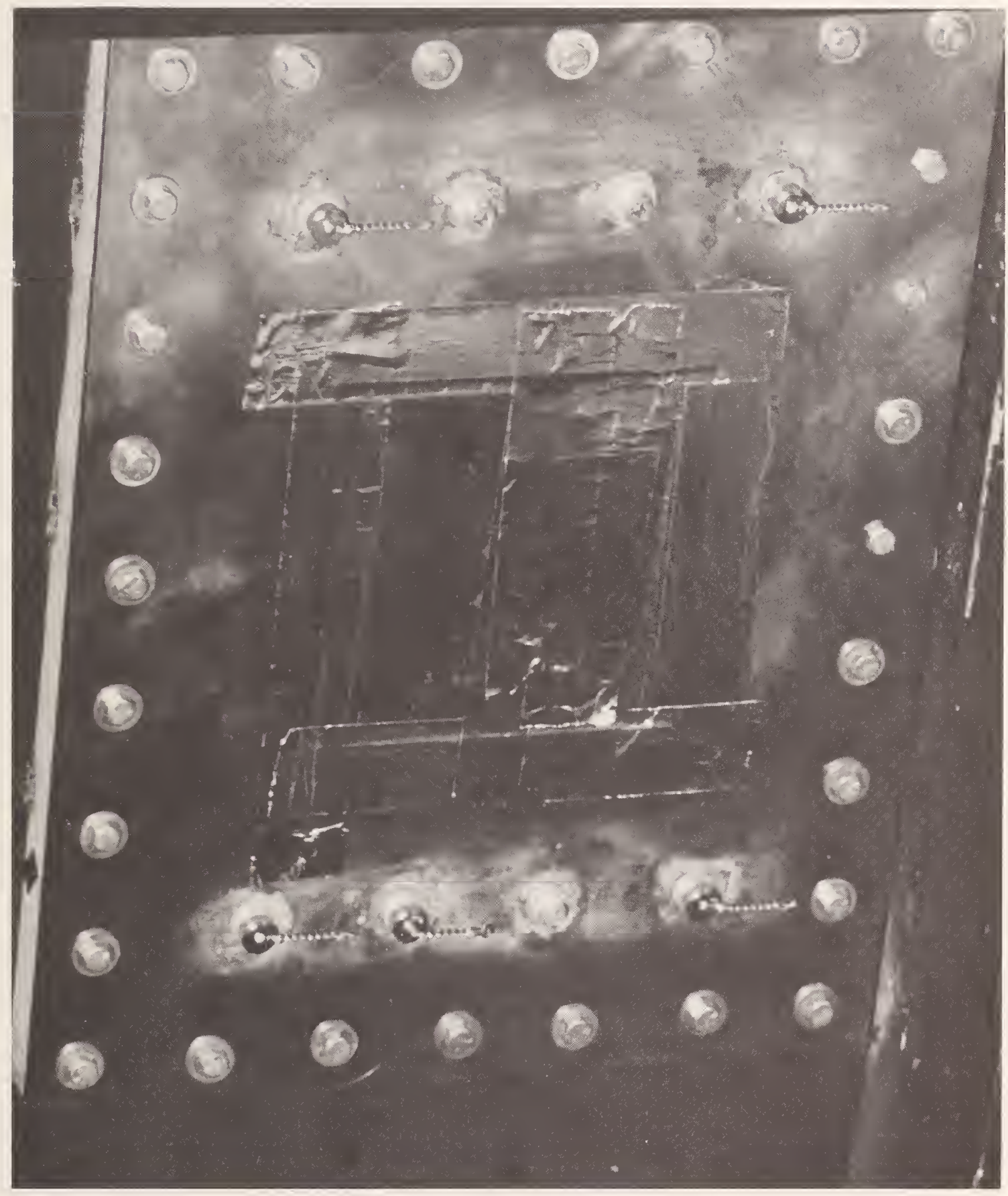

Figure 2.14 Bulkhead panel used for accessing NBS reverberation chamber. 
Figure 2.15a Photograph of field tuner/stirrer installed in McDonnell Douglas Corporation $2.4 \mathrm{~m} \times 3.0 \mathrm{~m} \times 7.6 \mathrm{~m}$ TEMEC

(reverberation chamber) facility.

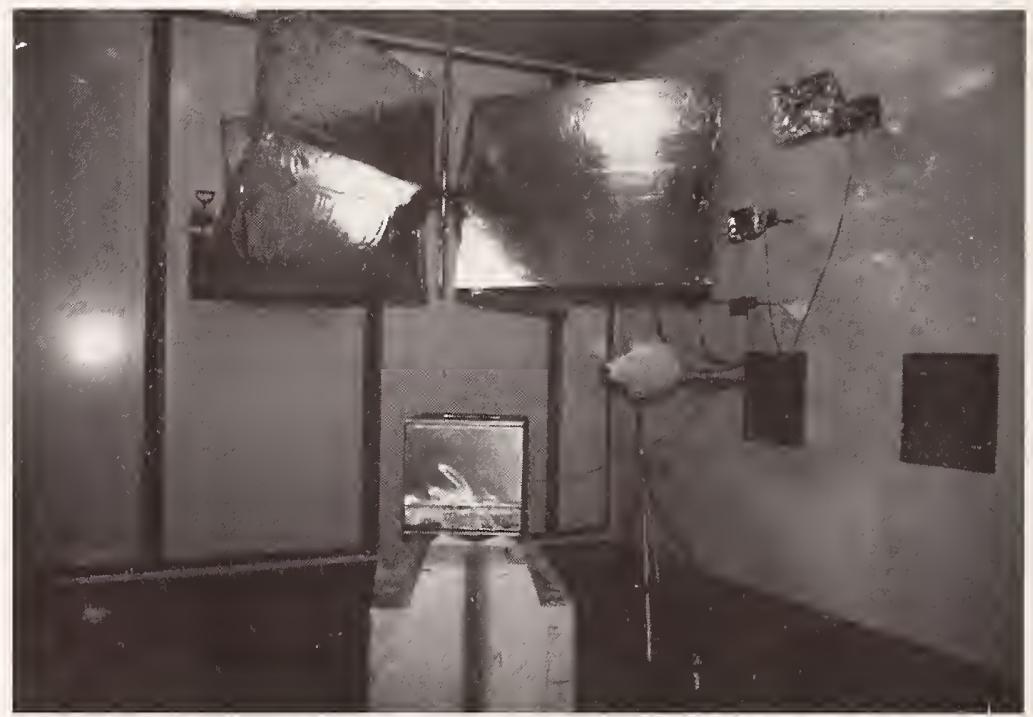

Figure 2.15b Photograph of field tuner/stirrer installed in NSWC $3.5 \mathrm{~m} \times 5.2 \mathrm{~m} \times 5.9 \mathrm{~m}$ reverberation chamber. 


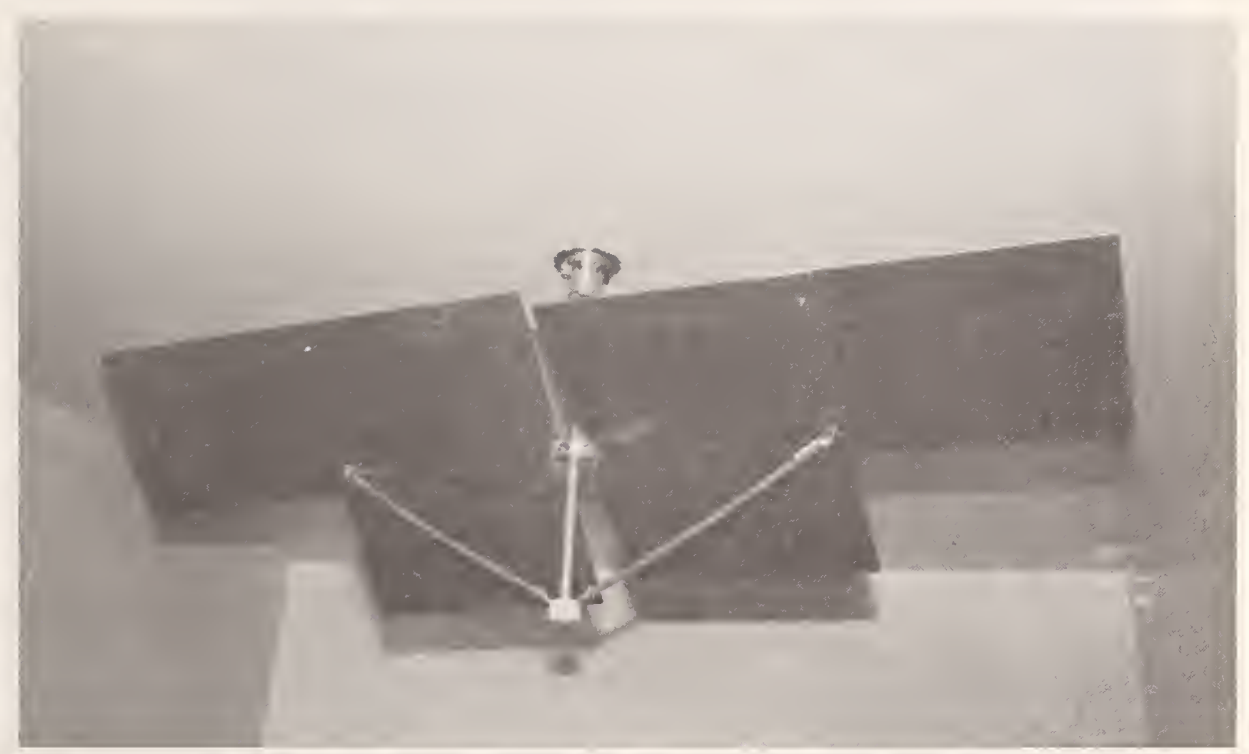

Figure 2.15c. Photograph of field tuner/stirrer installed in NBS $2.7 \mathrm{~m} \times 3.1 \mathrm{~m} \mathrm{x} 4.6 \mathrm{~m}$ reverberation chamber.

Figure 2.15. Tuner designs for use in reverberation chambers a) McDonnell Douglas Corp。TEMEC tuner, b) NSWC chamber tuner, c) NBS chamber tuner. 


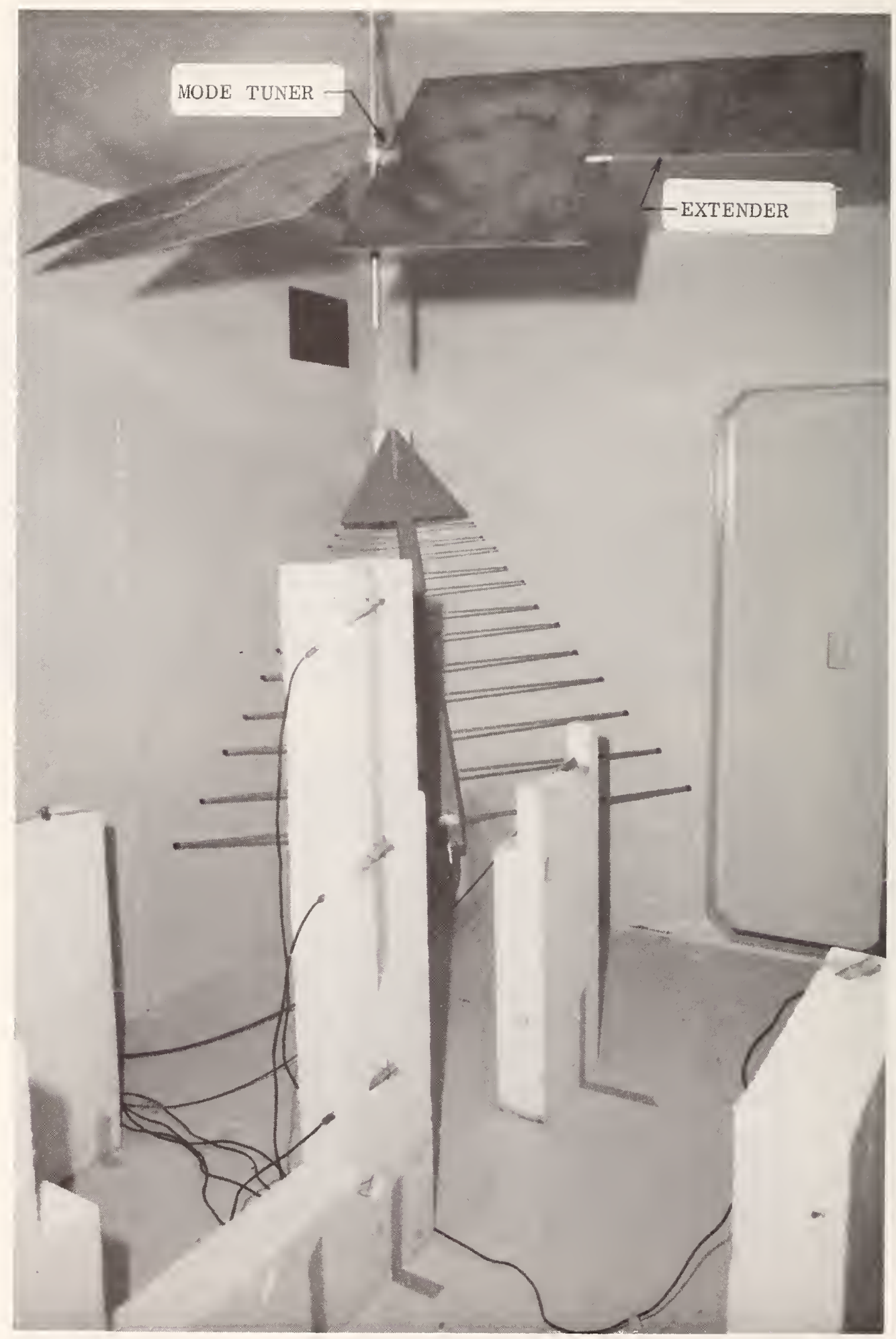

Figure 2.16 Photograph of tuner, log-periodic transmitting antenna and NBS isotropic probes inside NBS reverberation chamber. 


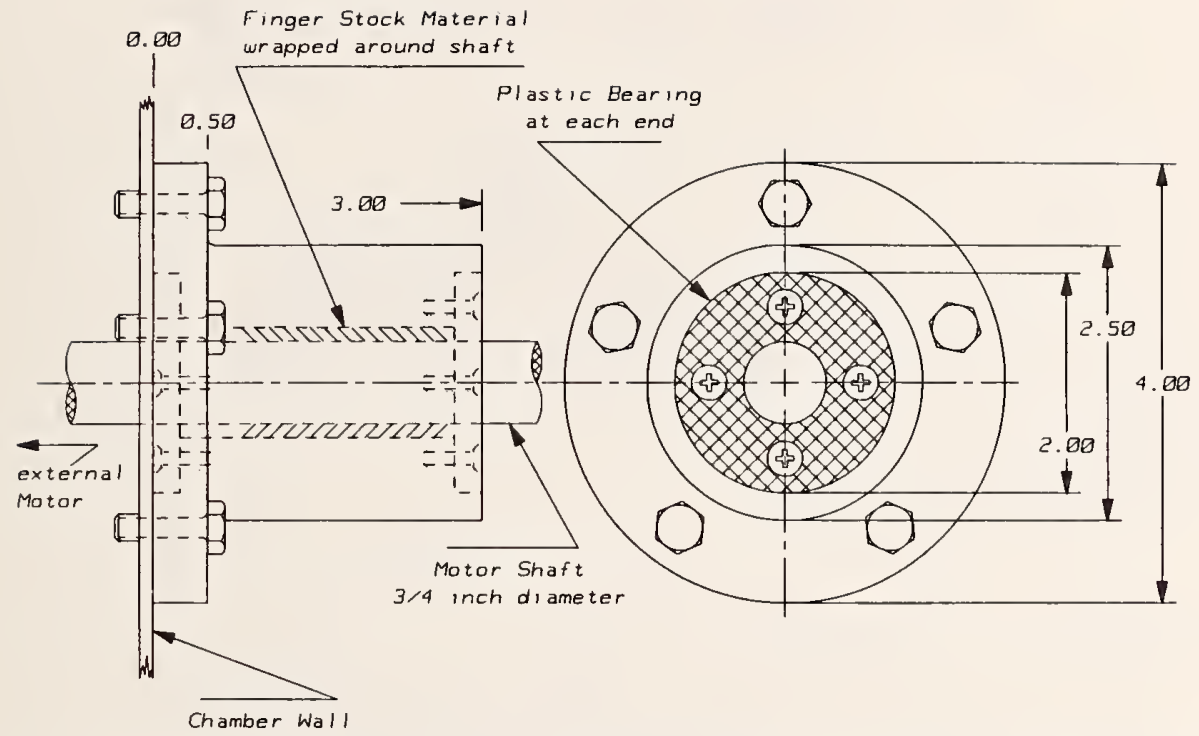

Figure 2.17a. Details of collet for mounting tuner shaft to motor through wall of reverberation chamber.

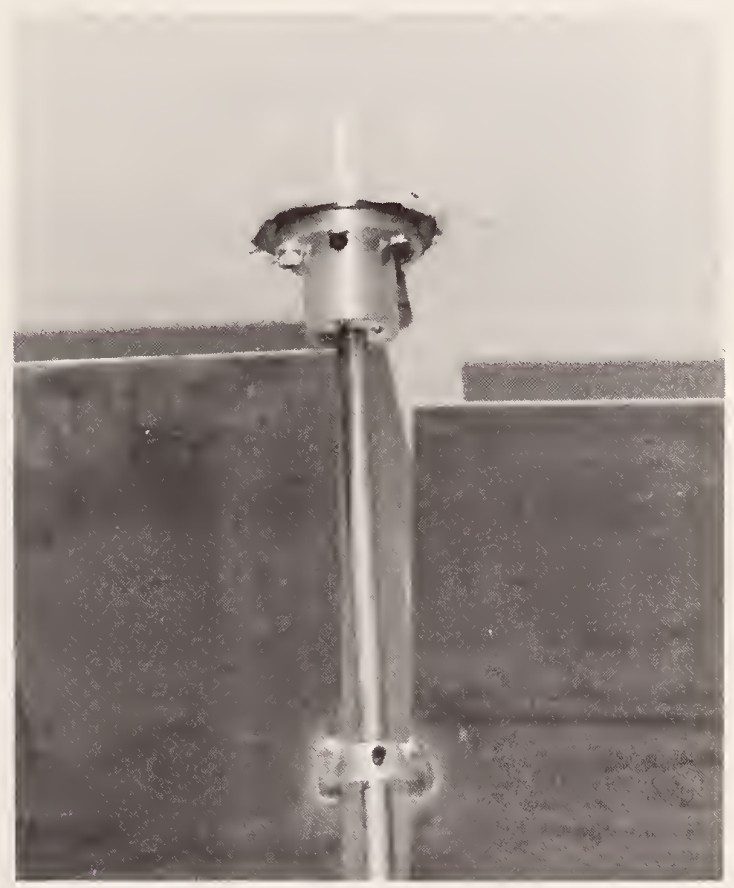

Figure 2.17b. Photograph of tuner shaft mounted through collet on ceiling of NBS reverberation chamber.

Figure 2.17 Details of tuner and stepping motor mounting for the NBS reverberation chamber. 

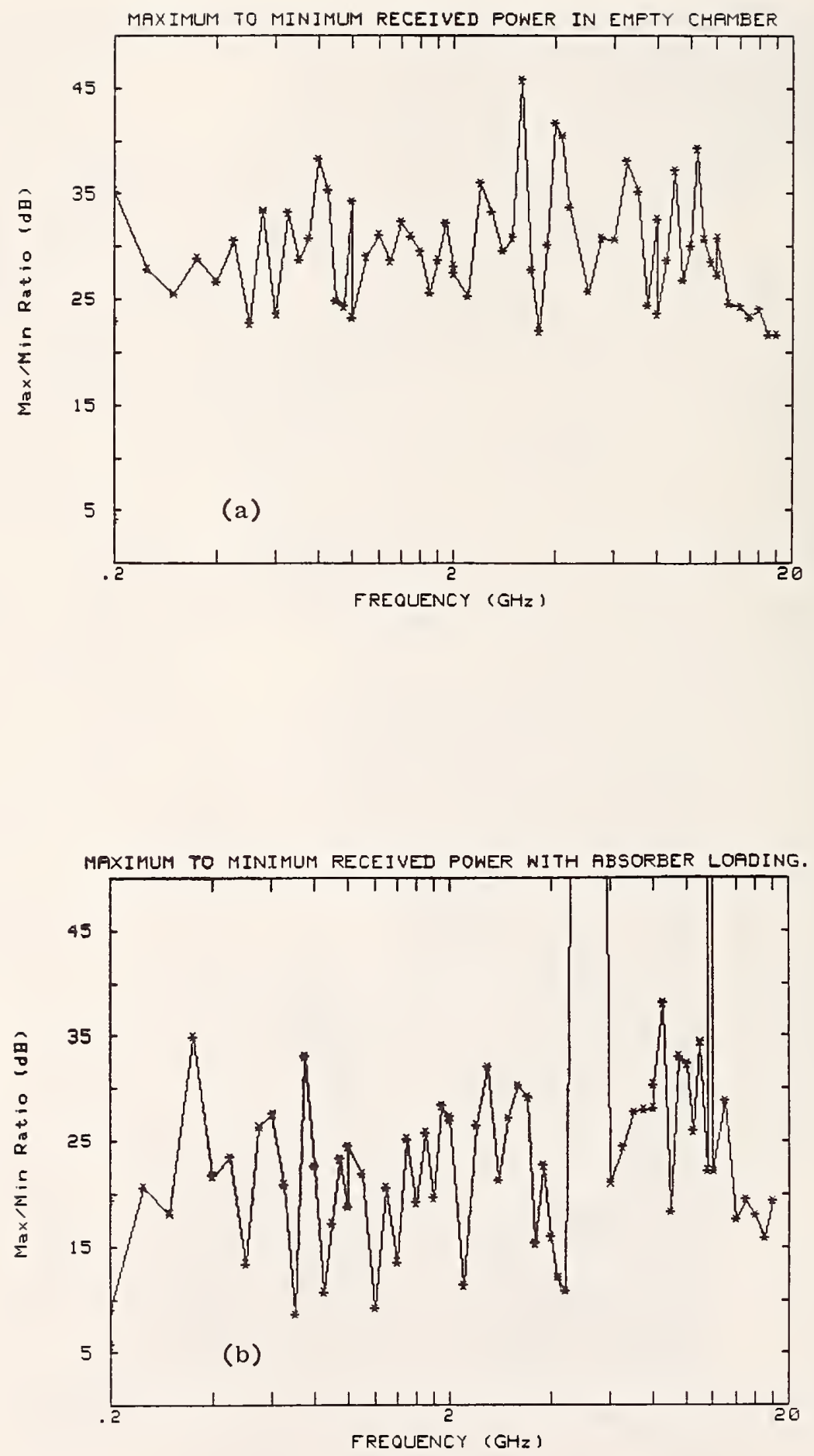

Figure 2.18 Ratio of maximum to minimum received power obtained by rotating tuner in the frequency range $200 \mathrm{MHz}$ to $18 \mathrm{GHz}$. a) Empty chamber, b) Four pieces of $66 \mathrm{~cm} \mathrm{rf}$ absorber placed upright in center of chamber, $0.5 \mathrm{~m}$ above floor. 

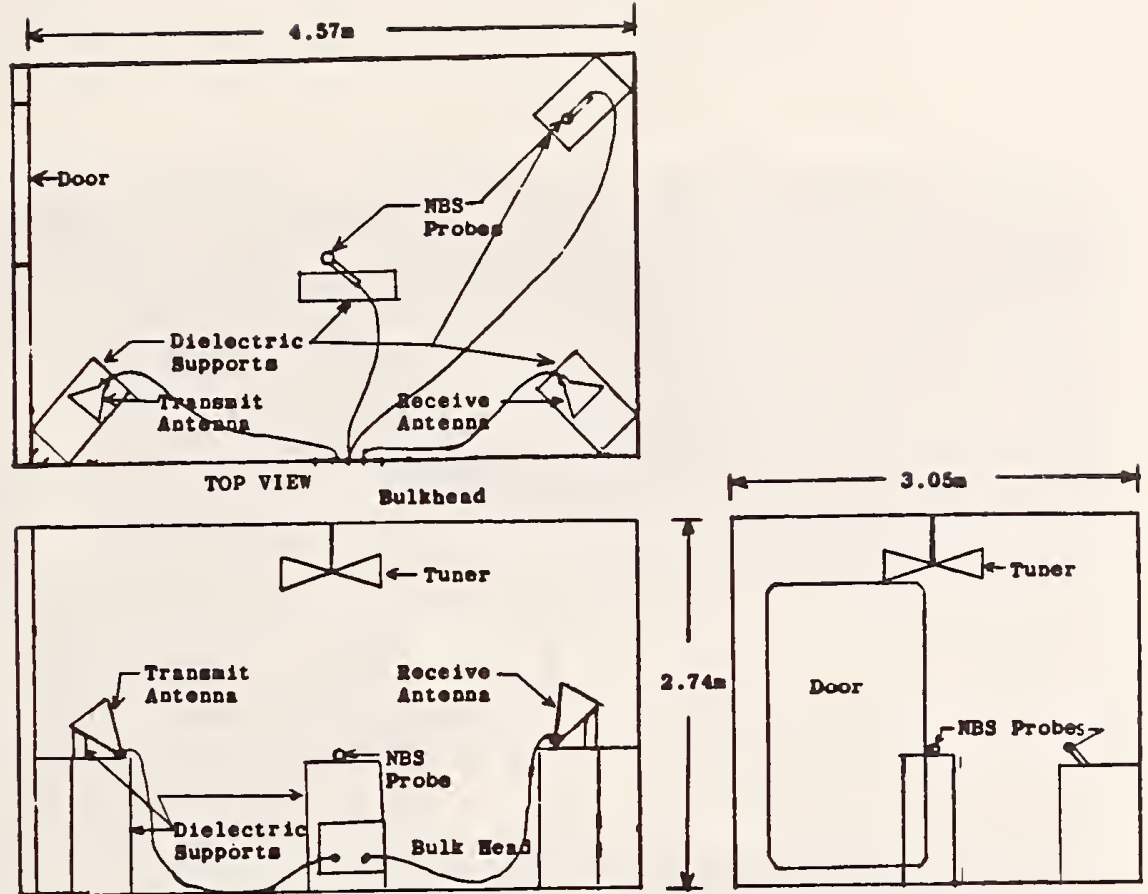

OIDE VISt

IND VITI

Cross sectional views of NBS reverberation chamber for use at frequencies

Figure 2.19 a. Cross $1.0 \mathrm{GHz}$ showing location of antenna and NBS probes.

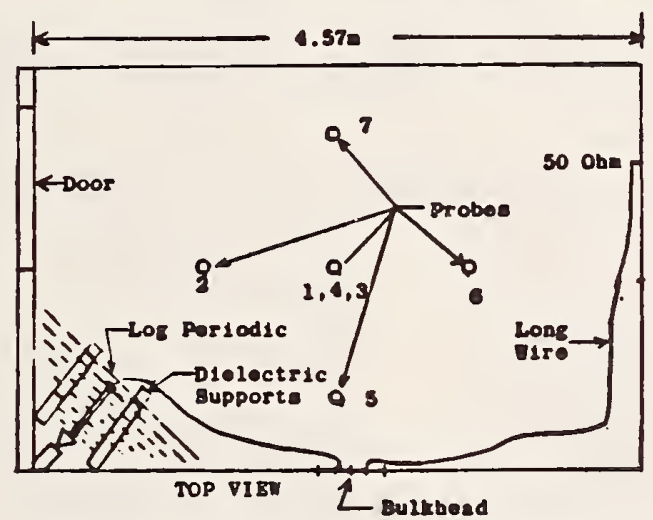

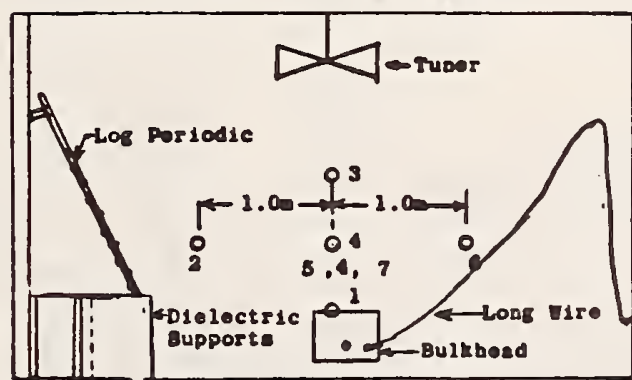

OIDE VIIII

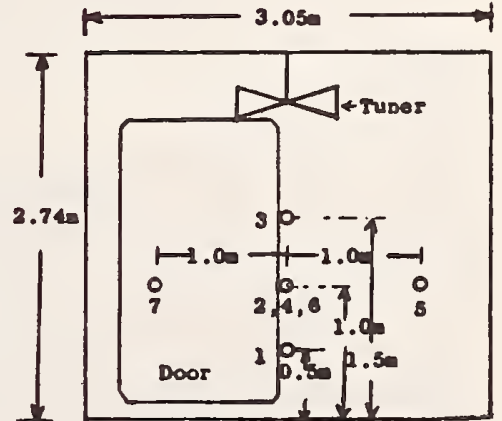

IST VIIII

Figure 2.19b. Cross sectional views of NBS reverberation chamber for use at frequencies below $1.0 \mathrm{GHz}$ showing location of antenna and array of NBS próbes.

Figure 2.19 Cross sectional views of NBS reverberation chamber showing placement of tuner, transmitting and receiving antennas, and NBS probes. 


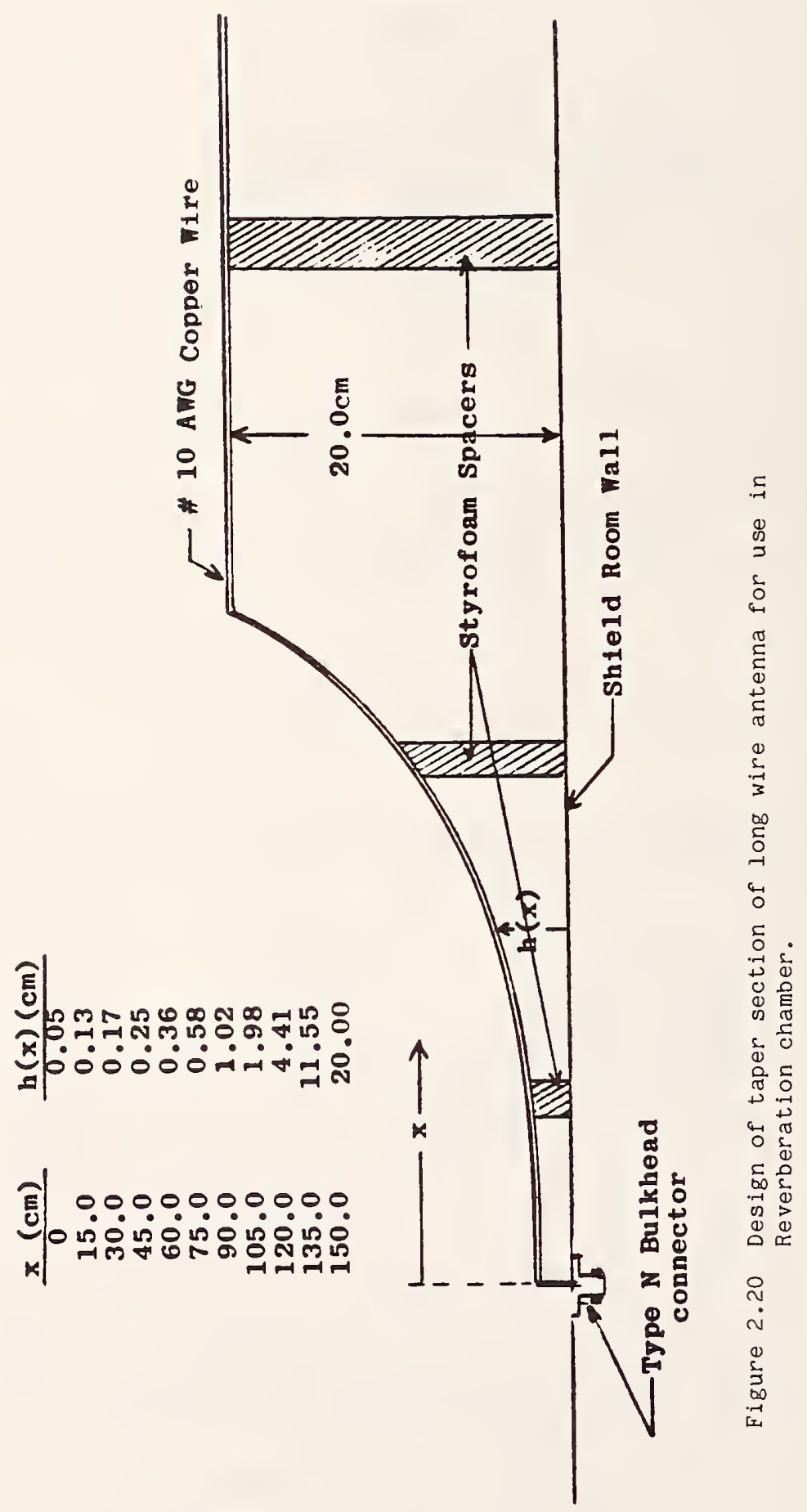




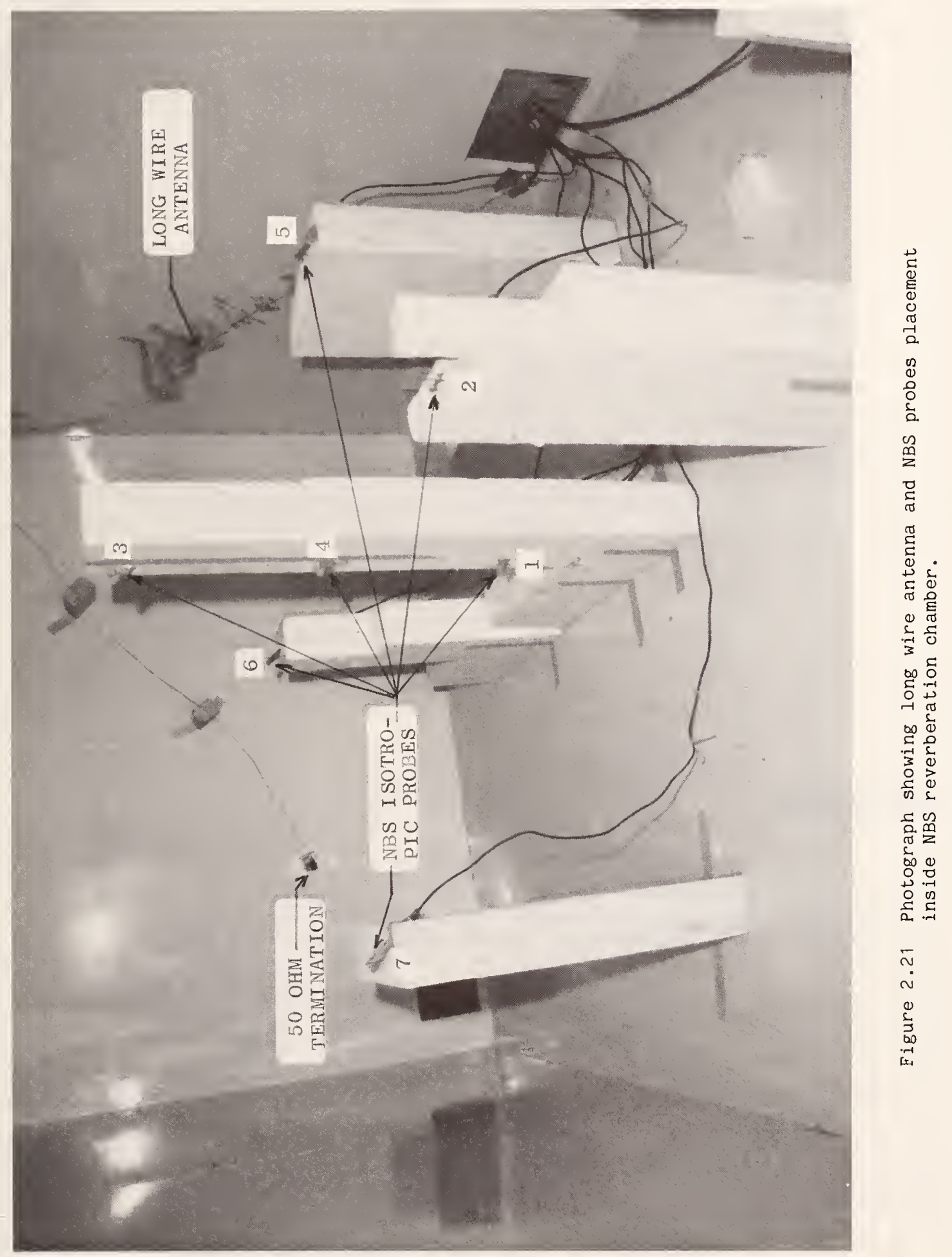



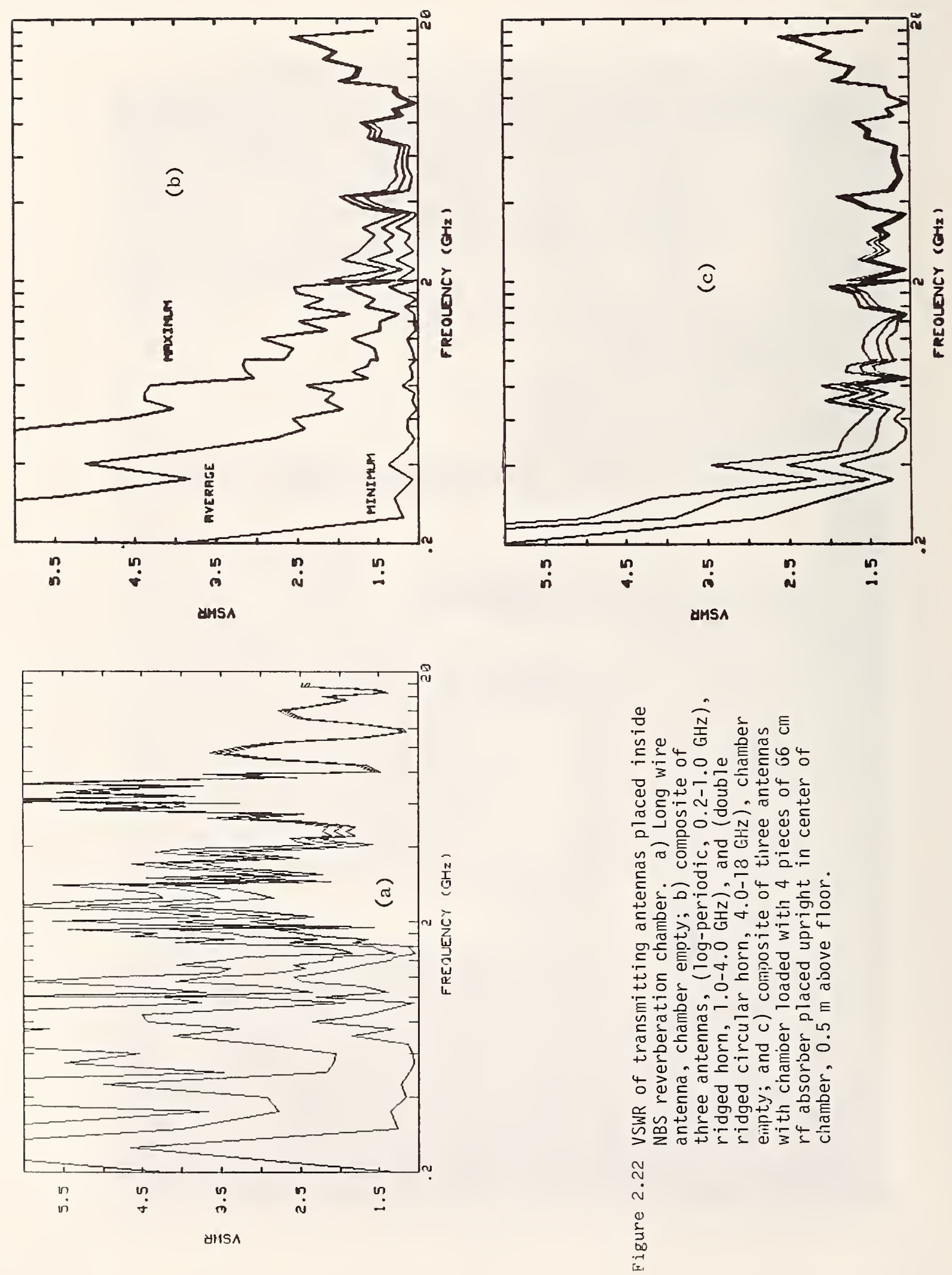


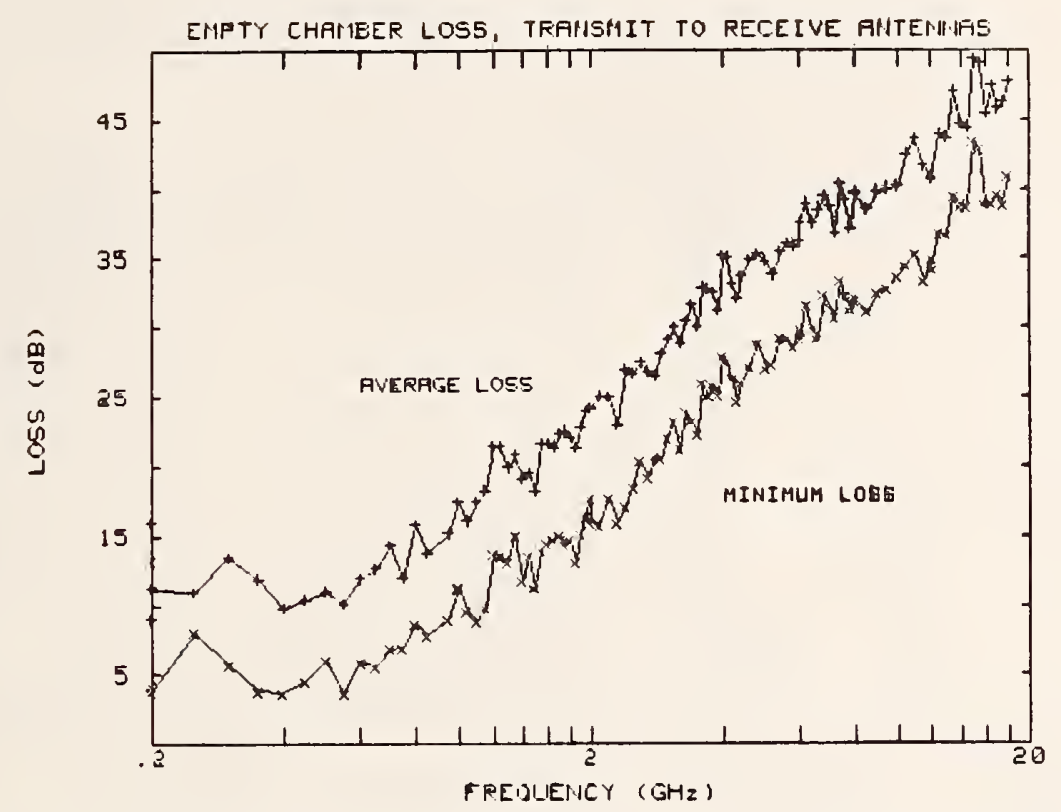

Figure 2.23 Average and minimum losses between transmitted and received powers measured at antennas's terminals inside NBS reverberation chamber. Long wire antenna transmitting (200 $\mathrm{MHz}$ - $18 \mathrm{GHz}$ ), composite of three antenna receiving (log periodic $200 \mathrm{MHz}-1000 \mathrm{MHz}$, ridged horn, $1.0 \mathrm{GHz}-4.0 \mathrm{GHz}$, double ridged circular horn, $4.0 \mathrm{GHz}-18 \mathrm{GHz}$ ).

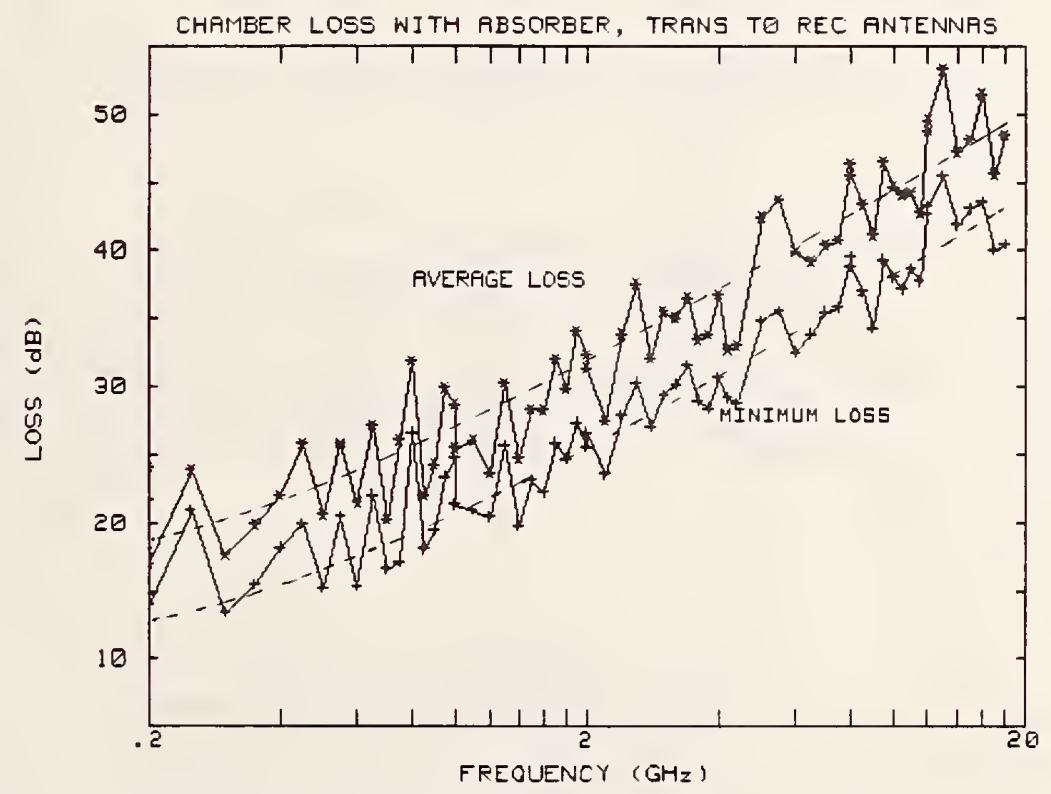

Figure 2.24 Average and minimum losses between transmitted and received powers measured at antennas' terminals inside NBS chamber with four pieces of $66 \mathrm{~cm}$ rf absorber upright in center of chamber, $0.5 \mathrm{~m}$ above floor. Composite of three antennas used for transmitting and receiving. 


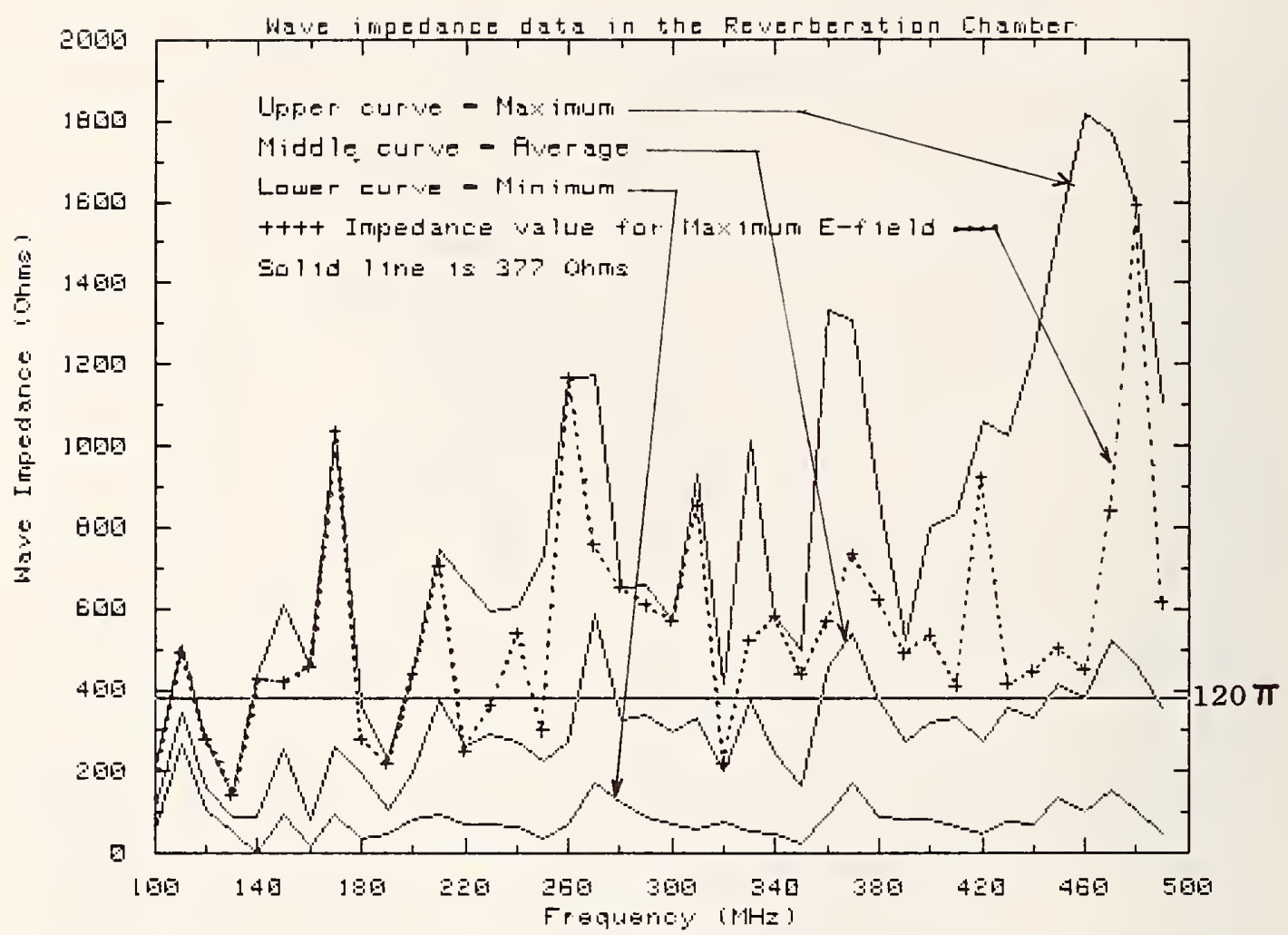

Figure 2.25 Magnitude of wave impedance inside NBS reverberation chamber.

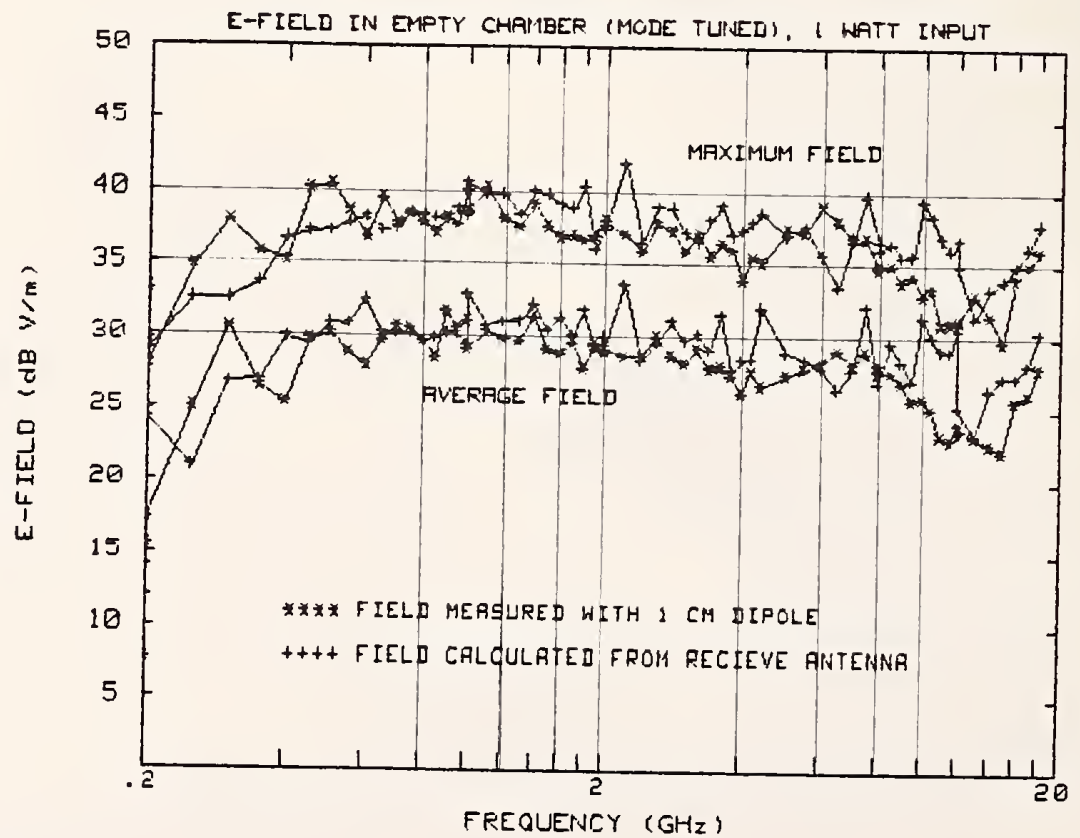

Figure 2.26 Average and maximum E-field strengths inside empty NBS reverberation chamber for $1 \mathrm{~W}$ net input power determined from: a) composite of 3 antennas received power measurements, and b) calibrated $1 \mathrm{~cm}$ dipole probe measurements. 

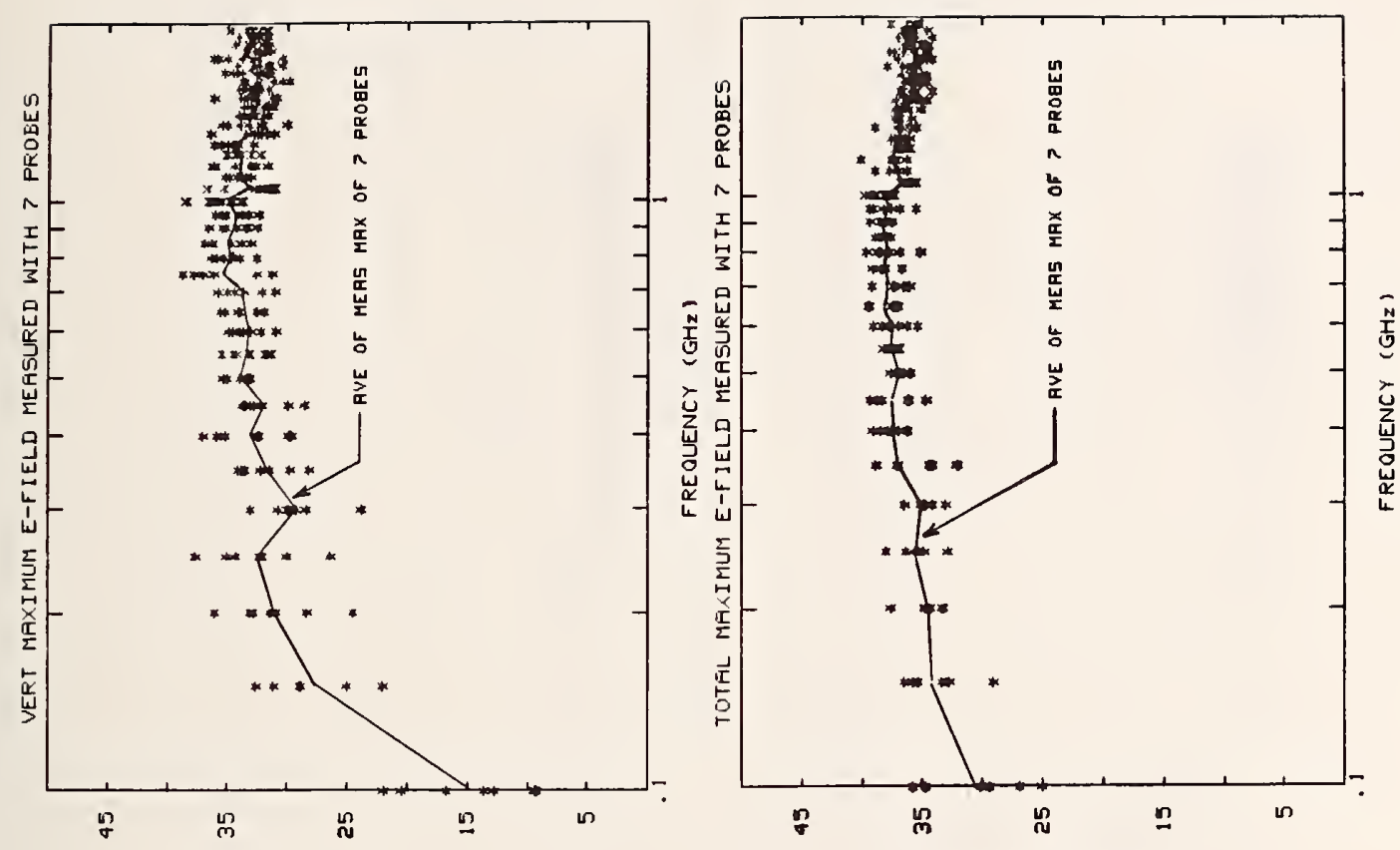

(w/A ap) वาI $y-3$
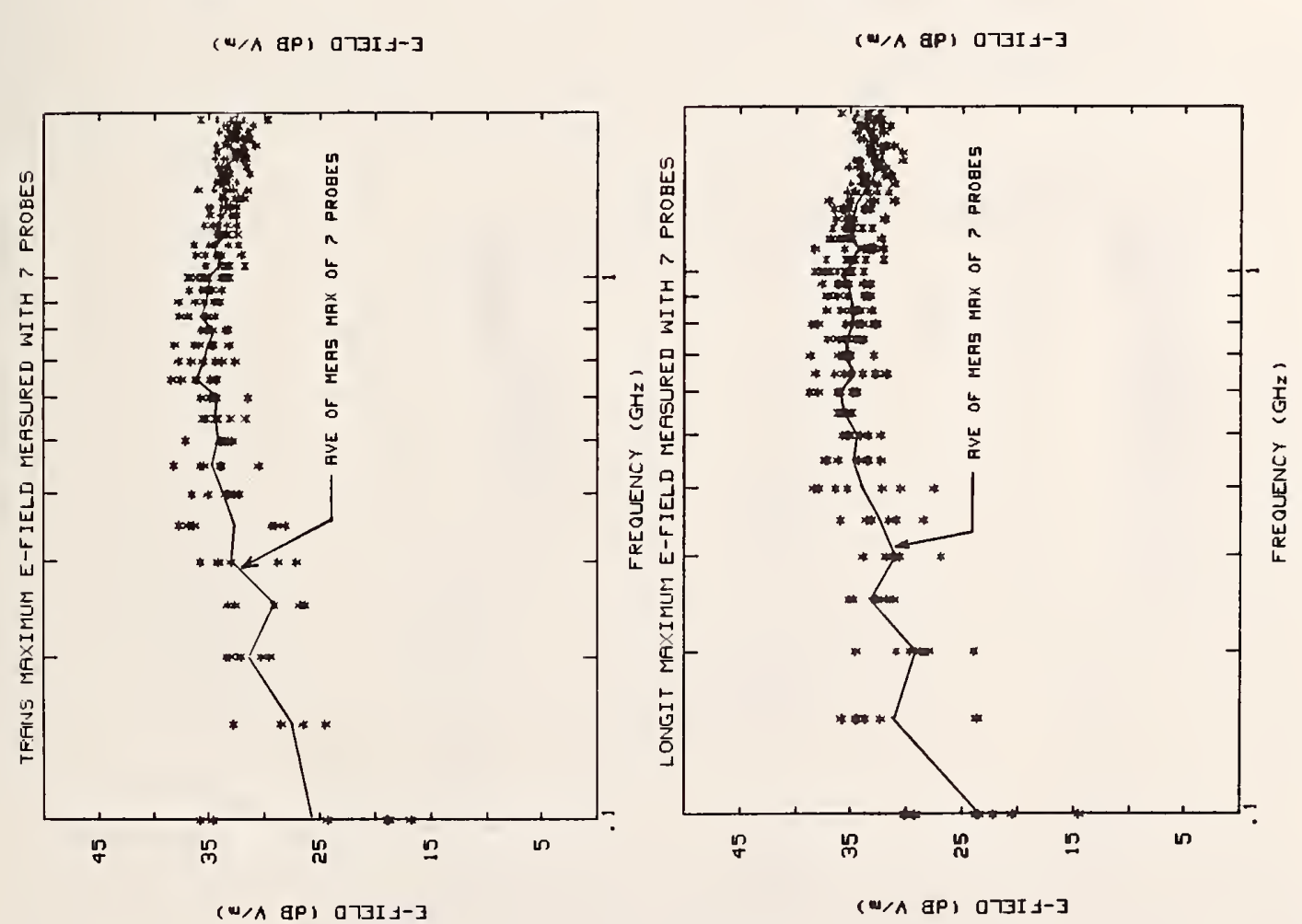


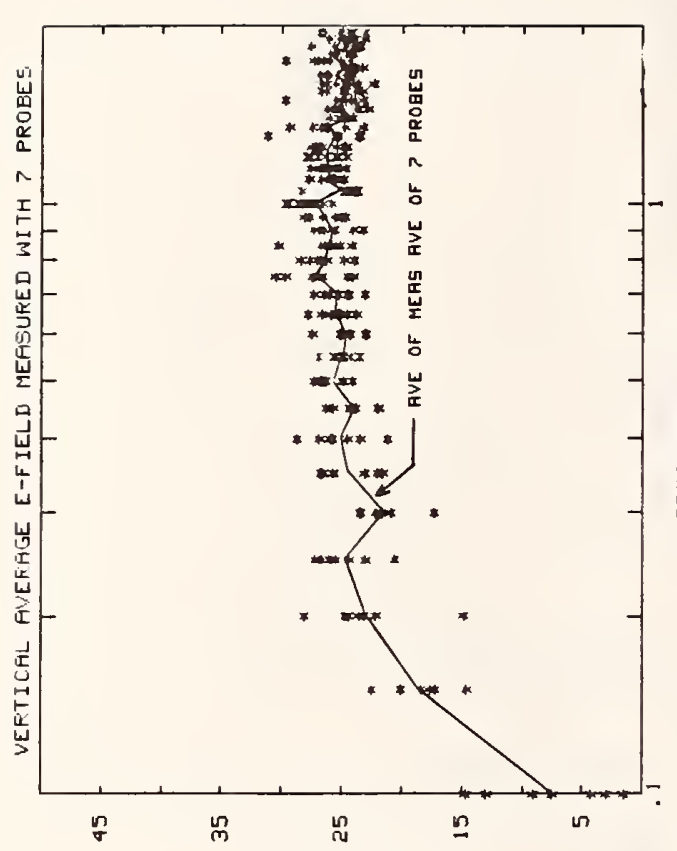

( $w / \wedge$ gP)

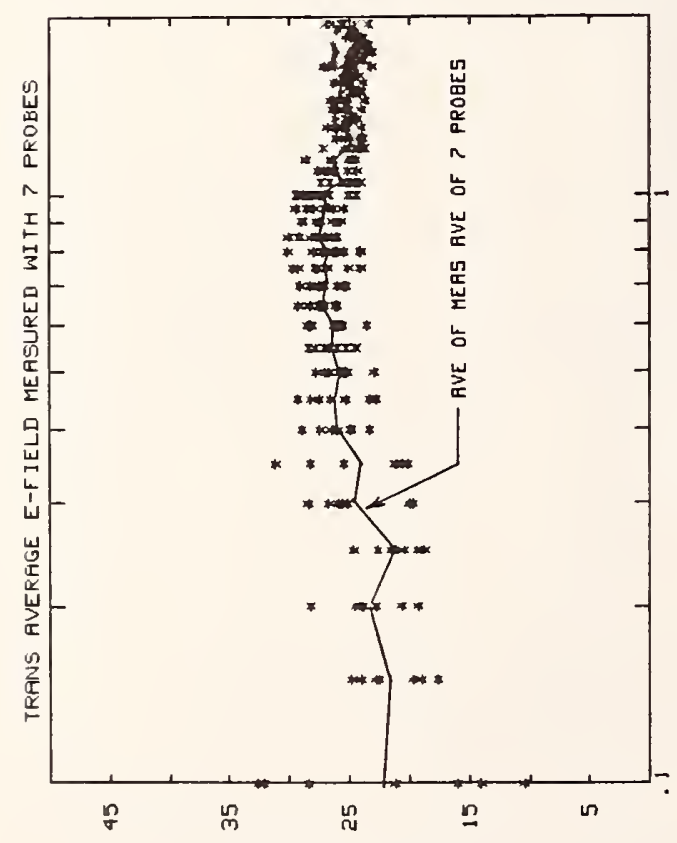

(w/A gP) gר $4-3$

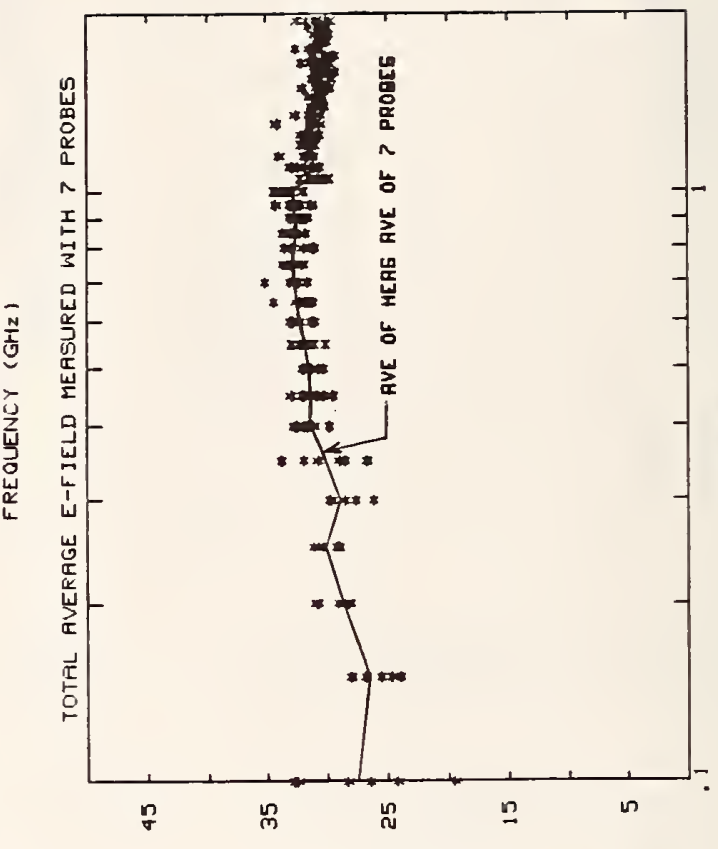

(w/1 gP) (4)

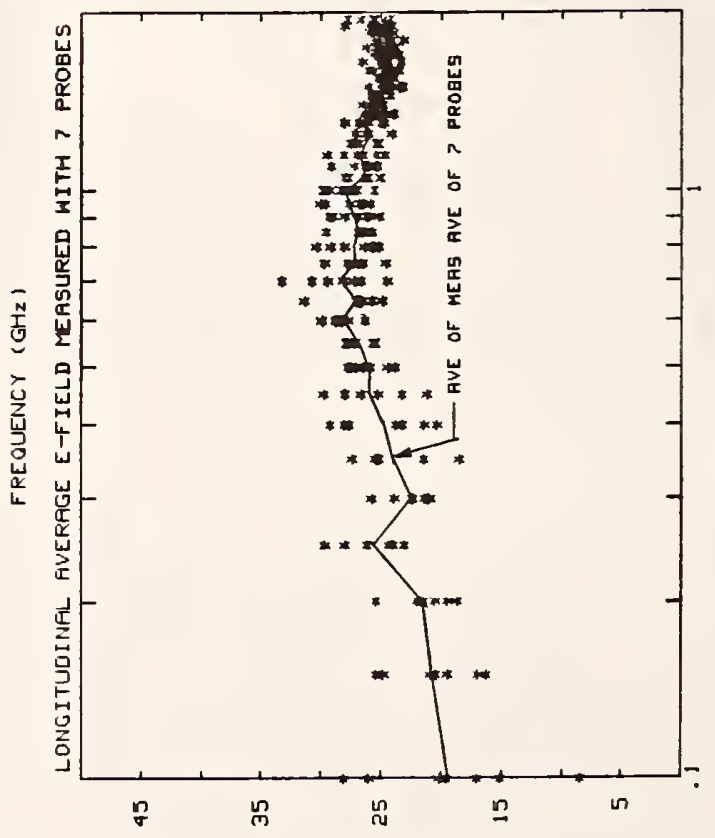

(4/^ gP) ОางI $y-3$

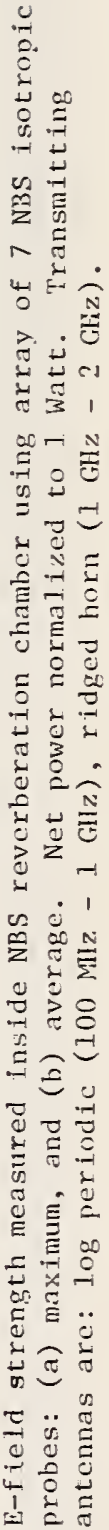

ָे 

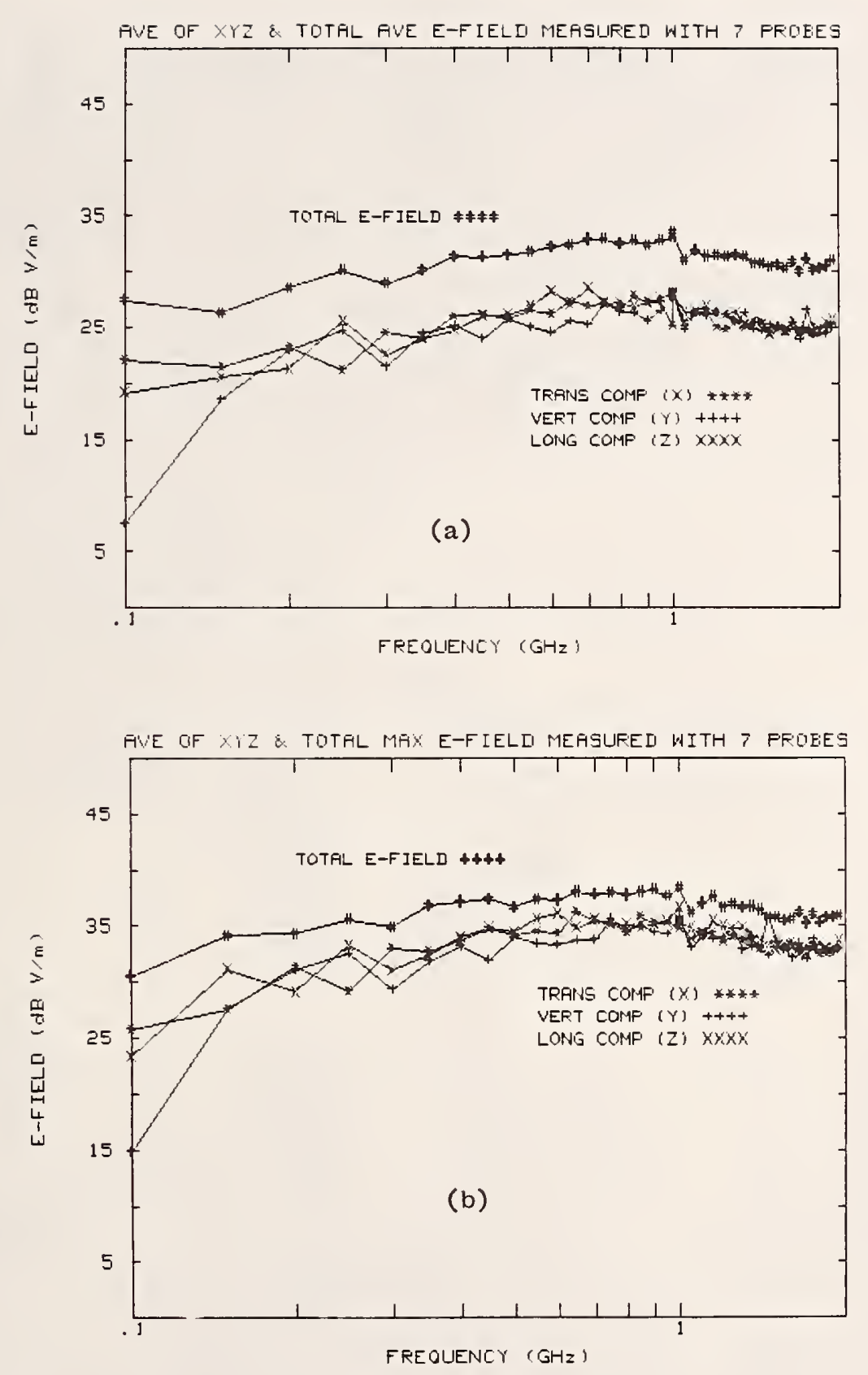

Figure 2.28 Mean values of the E-field strength measured inside NBS reverberation chamber using array of 7 NBS isotropic probes with 1 W net input power: (a) average, and (b) maximum. Data determined from maximum and average values of the 7 probes ( 7 locations) for the three orthogonal components and their sum (total) using 200 tuner positions ( 1.8 degree increments). 


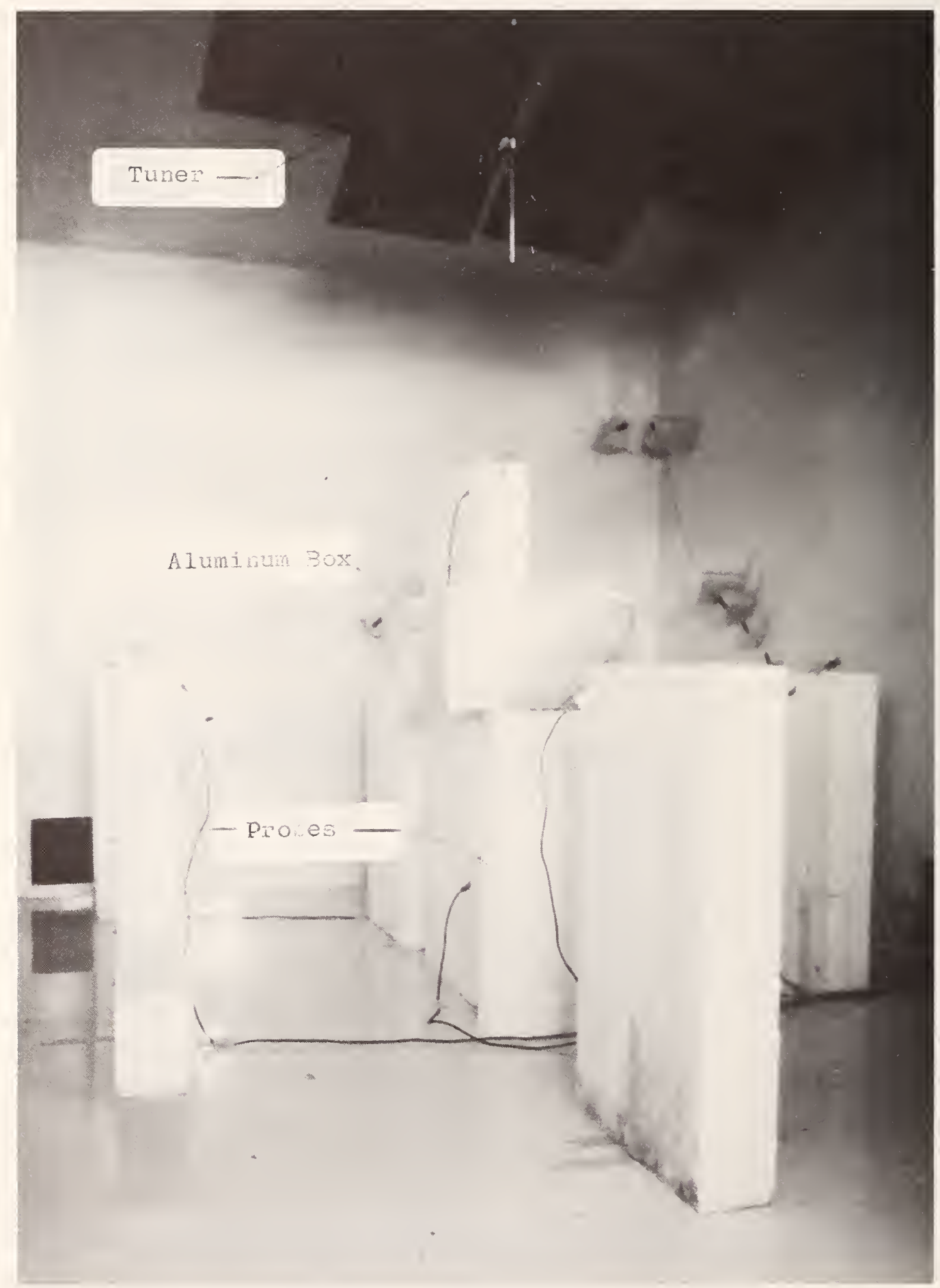

Figure 3.1a Photograph showing $30 \mathrm{~cm} \mathrm{x} 50 \mathrm{~cm} \times 60 \mathrm{~cm}$ welded aluminum box inside NBS reverberation chamber. 


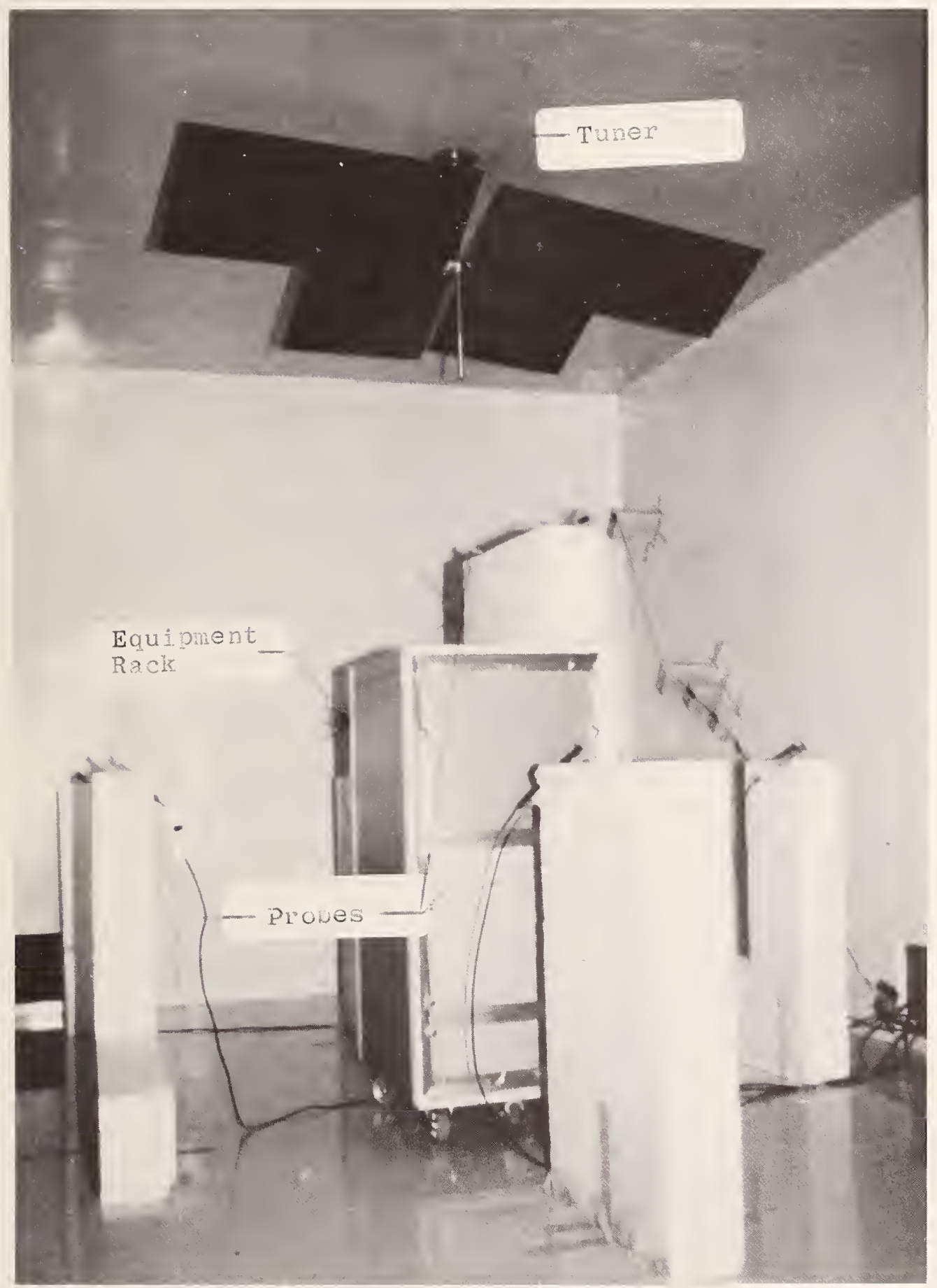

Figure 3.1b Photograph showing rolling equipment rack inside NBS reverberation chamber.

Figure 3.1 Placement of metallic objects inside NBS reverberation chamber to evaluate scattering effects upon E-field spatial distribution. a) Photograph showing $30 \mathrm{~cm} \times 50 \mathrm{~cm} \times 60 \mathrm{~cm}$ welded aluminum box inside NBS reverberation chamber. b) Photograph showing rolling equipment rack inside NBS reverberation chamber. 

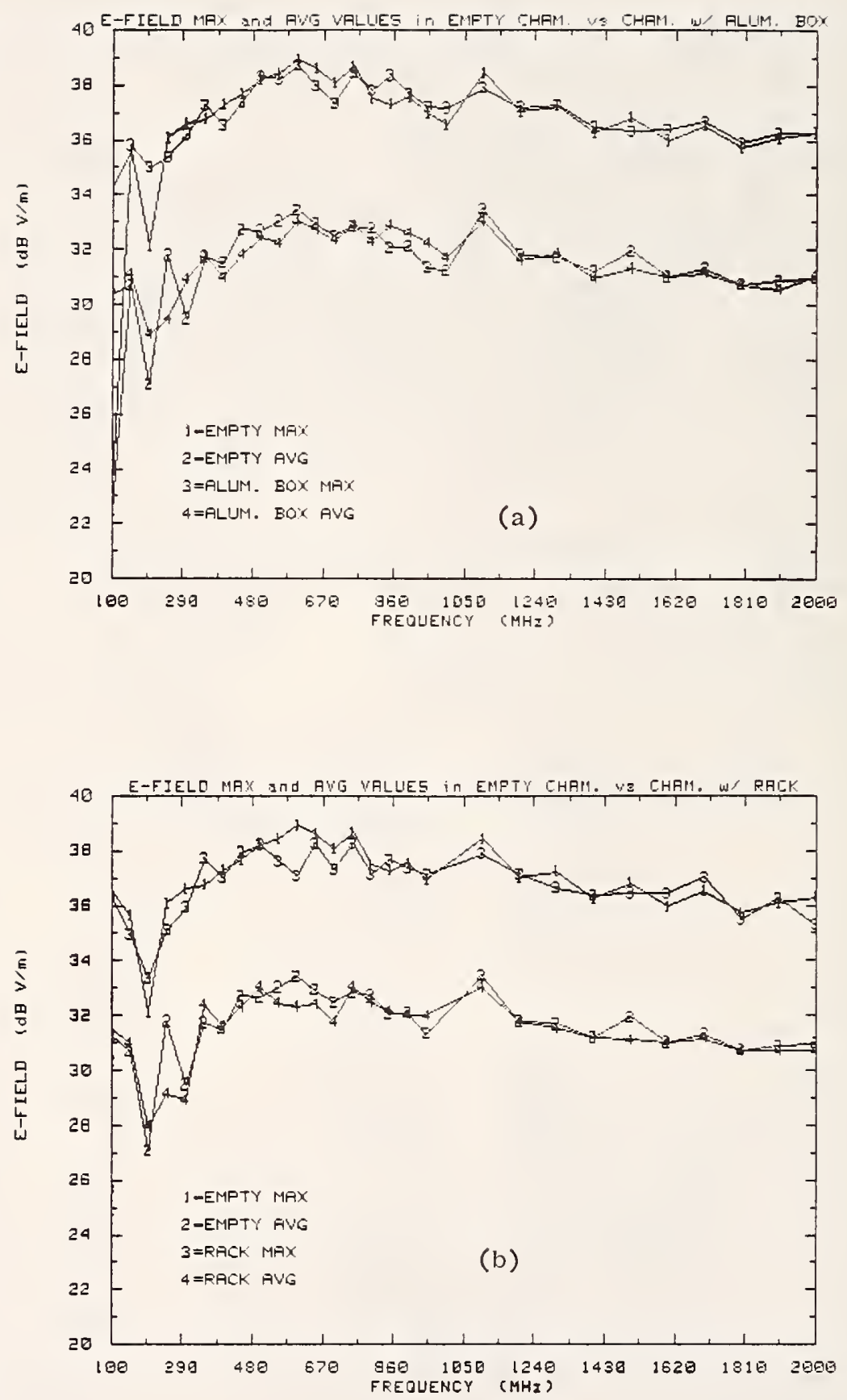

Figure 3.2 Comparison of the mean value of the average and maximum E-field strength measured using array of probes placed inside NBS reverberation chamber with chamber empty or with scattering object placed at center of test zone. a) $30 \mathrm{~cm} \times 50 \mathrm{~cm} \times 60 \mathrm{~cm}$ aluminum box, b) Rolling equipment rack. 


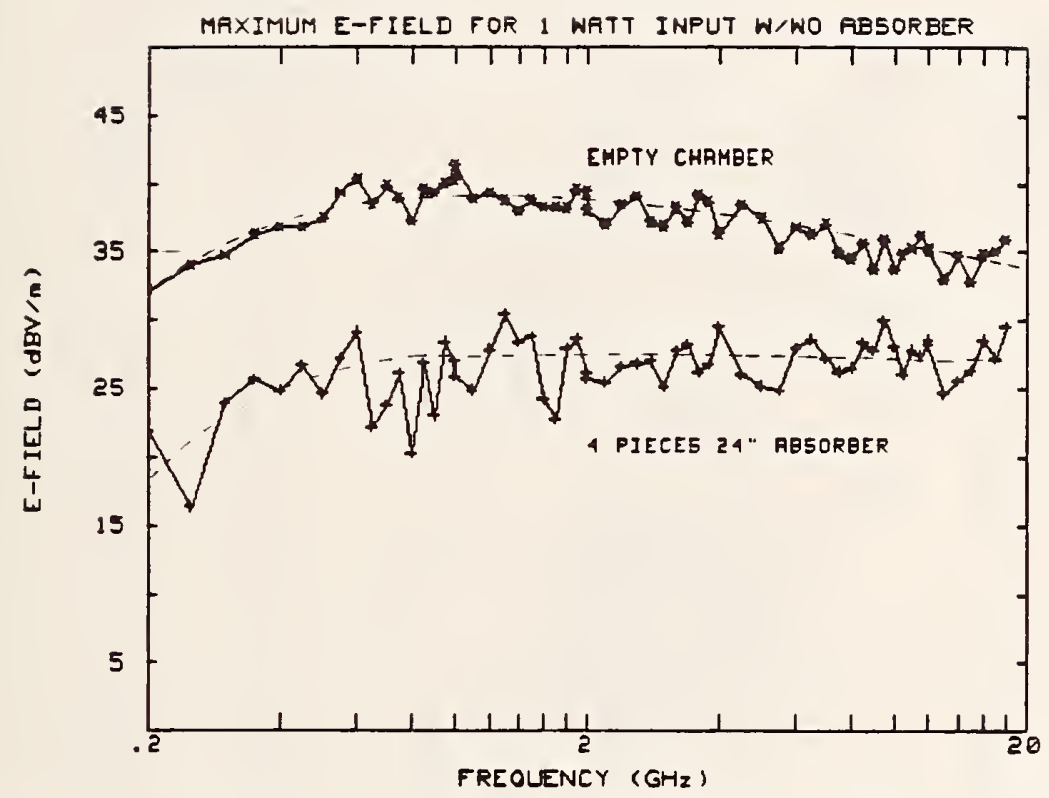

Figure 3.3 Maximum E-field strength inside empty chamber and chamber loaded with 4 pieces of $66 \mathrm{~cm} \mathrm{rf}$ absorber for $1 \mathrm{~W}$ net input power determined from composite of 3 antennas received power measurements. Data obtained using mode stirring approach. 


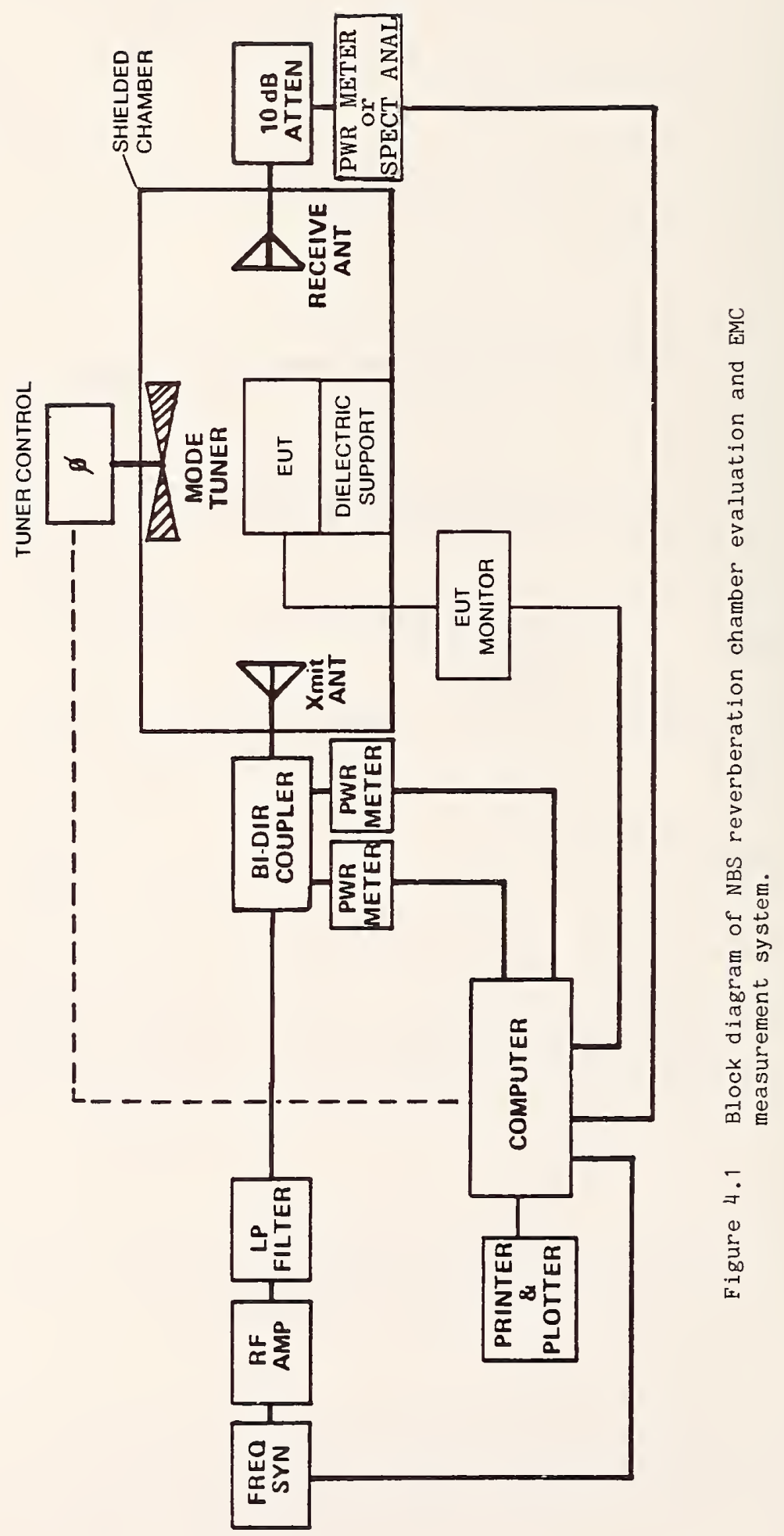




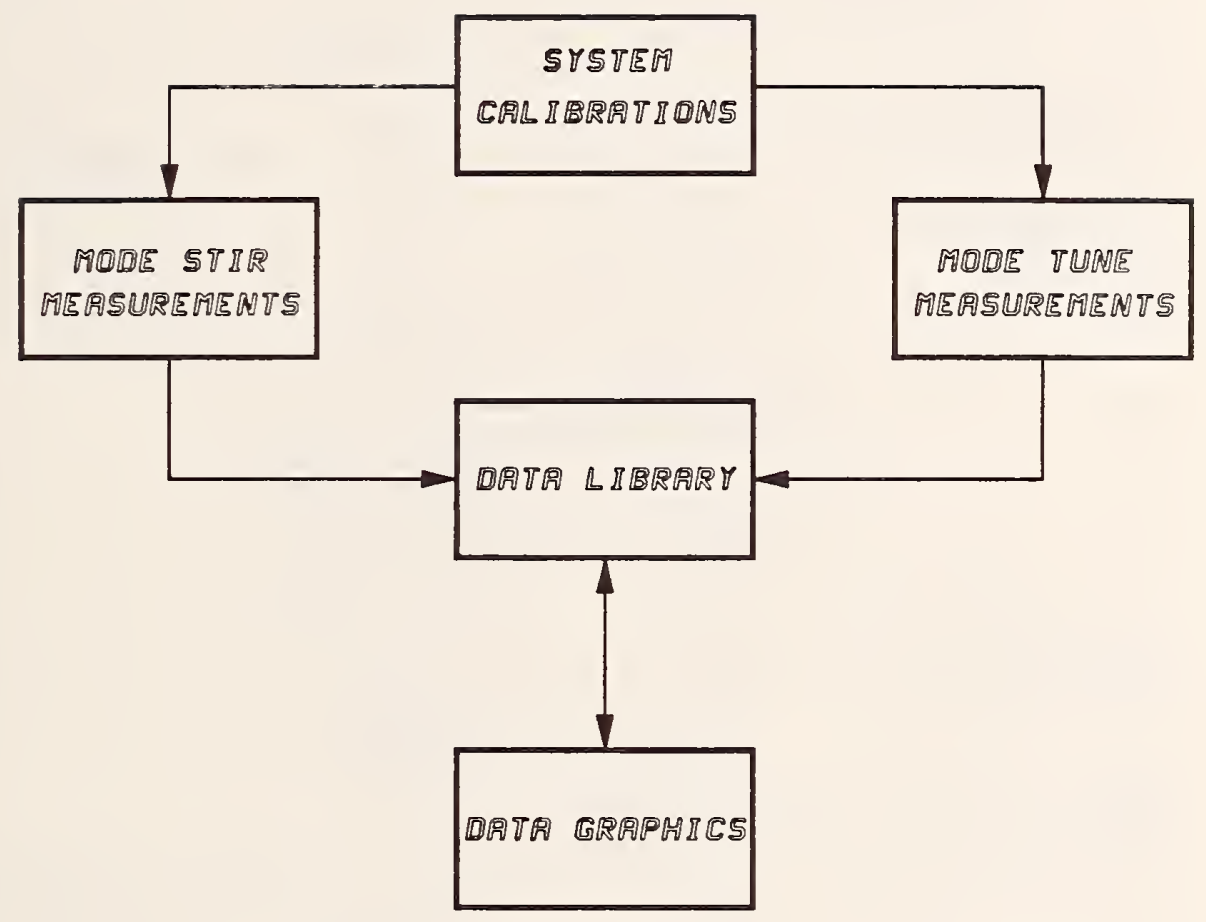

Figure 4.2 Major software tasks for performing and evaluation susceptibility tests using a reverberation chamber. 


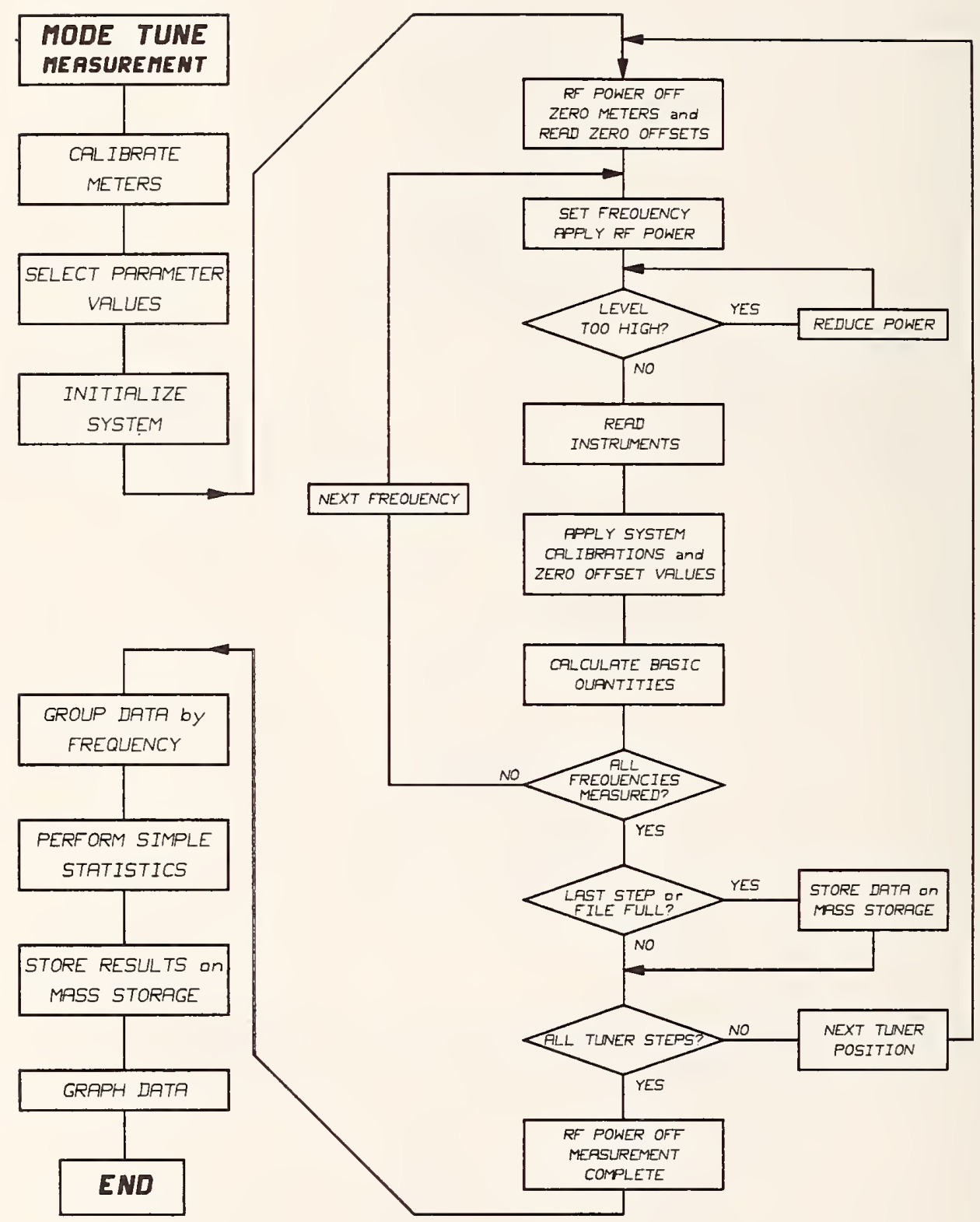

Figure 4.3 Flow diagram for mode-tuned measurements. 


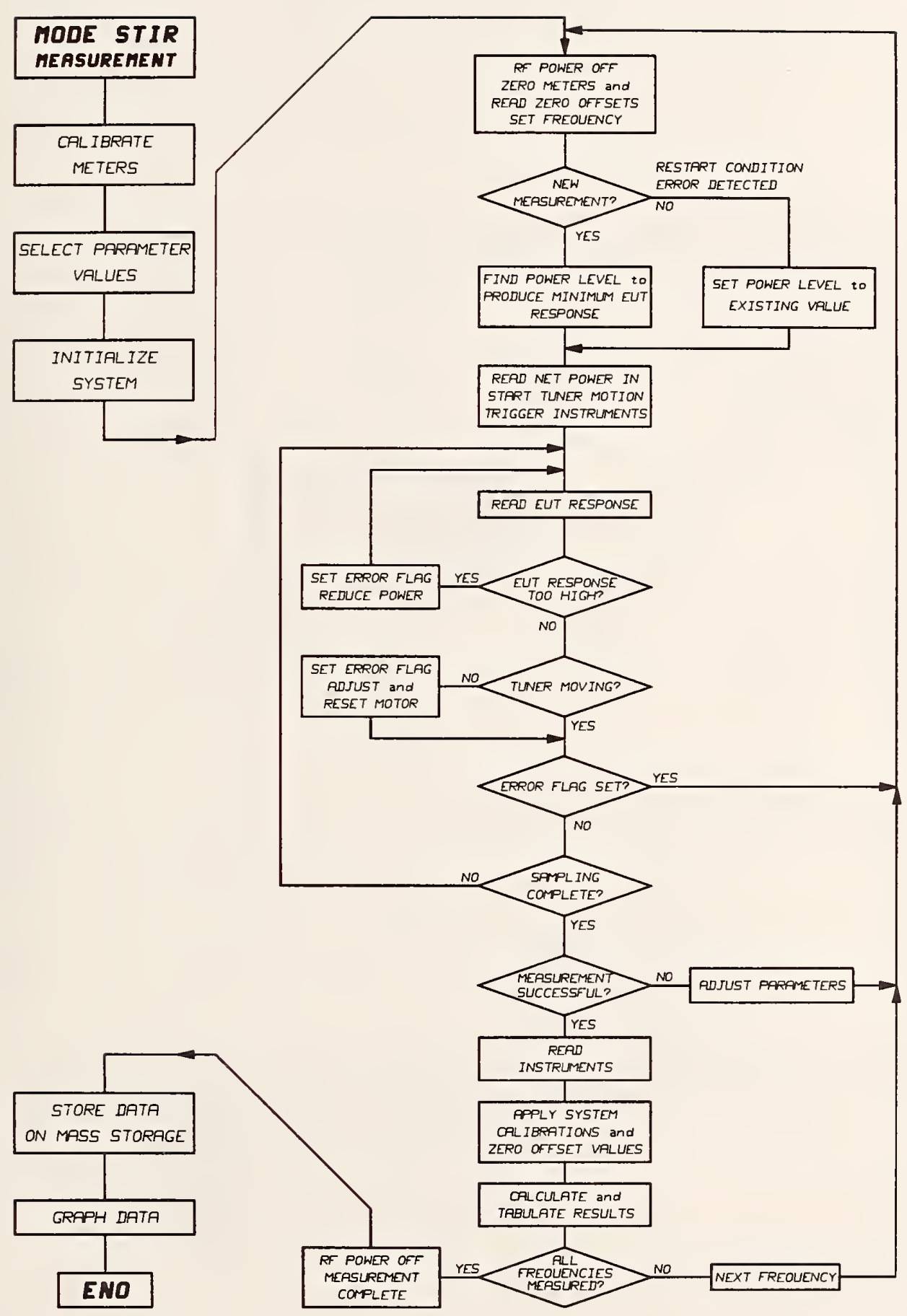

Figure 4.4 Flow diagram for mode-stirred measurements. 


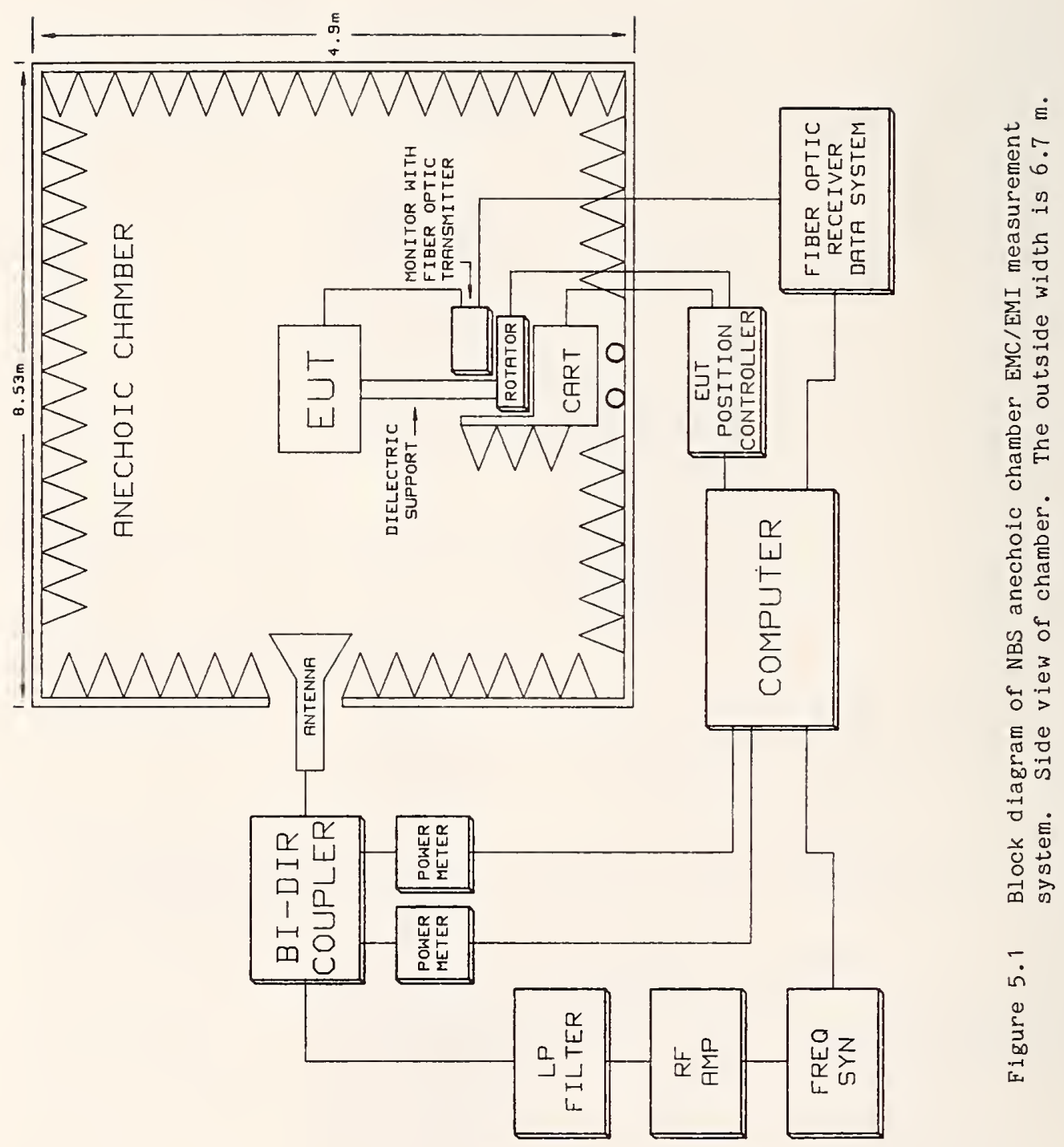




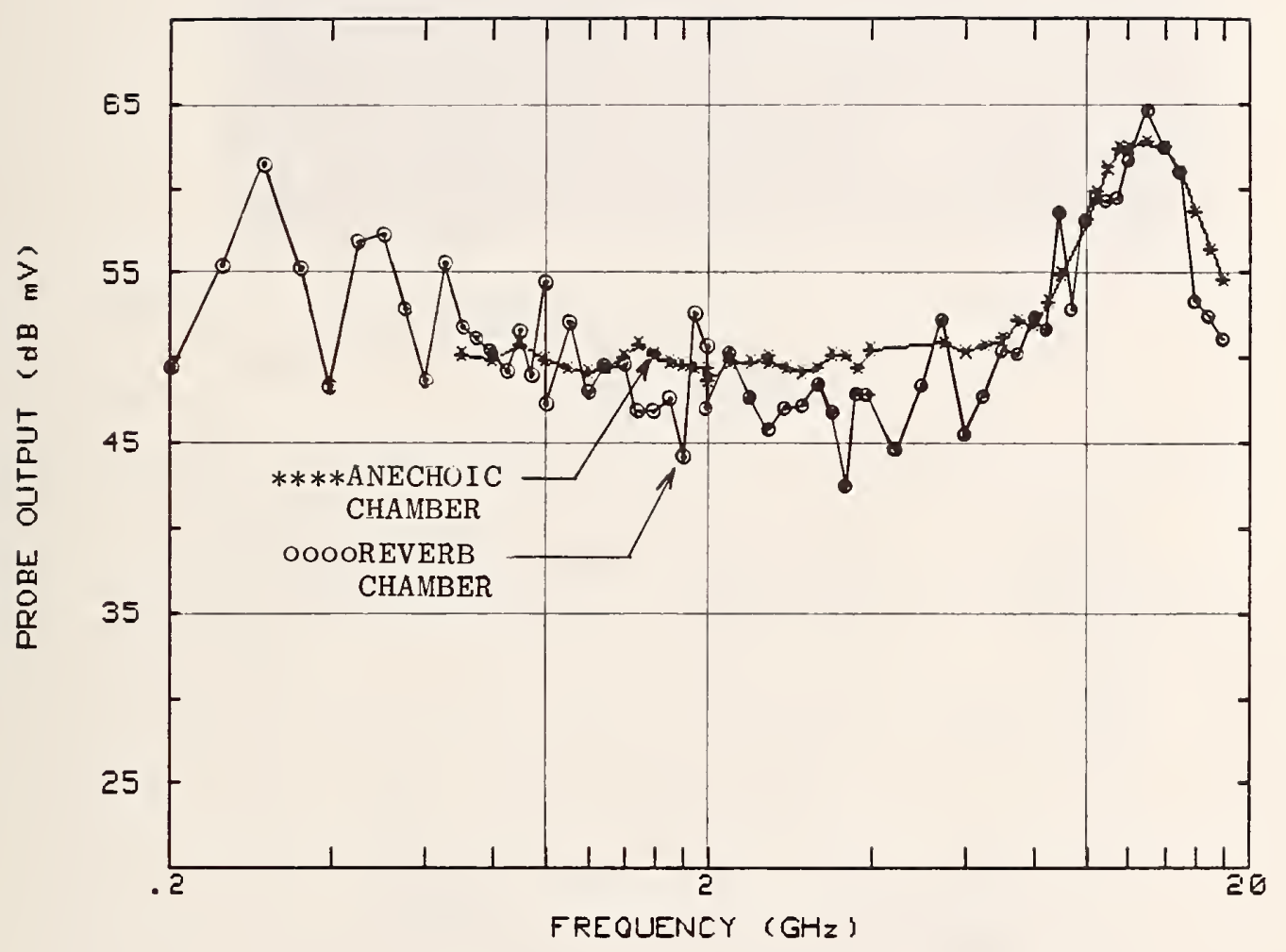

Figure 5.2 Comparison of $1 \mathrm{~cm}$ dipole probe's peak responses to EM field established inside NBS reverberation and anechoic chambers. Output normalized to E-field exposure of $37 \mathrm{~dB} \mathrm{~V} / \mathrm{m}$. 

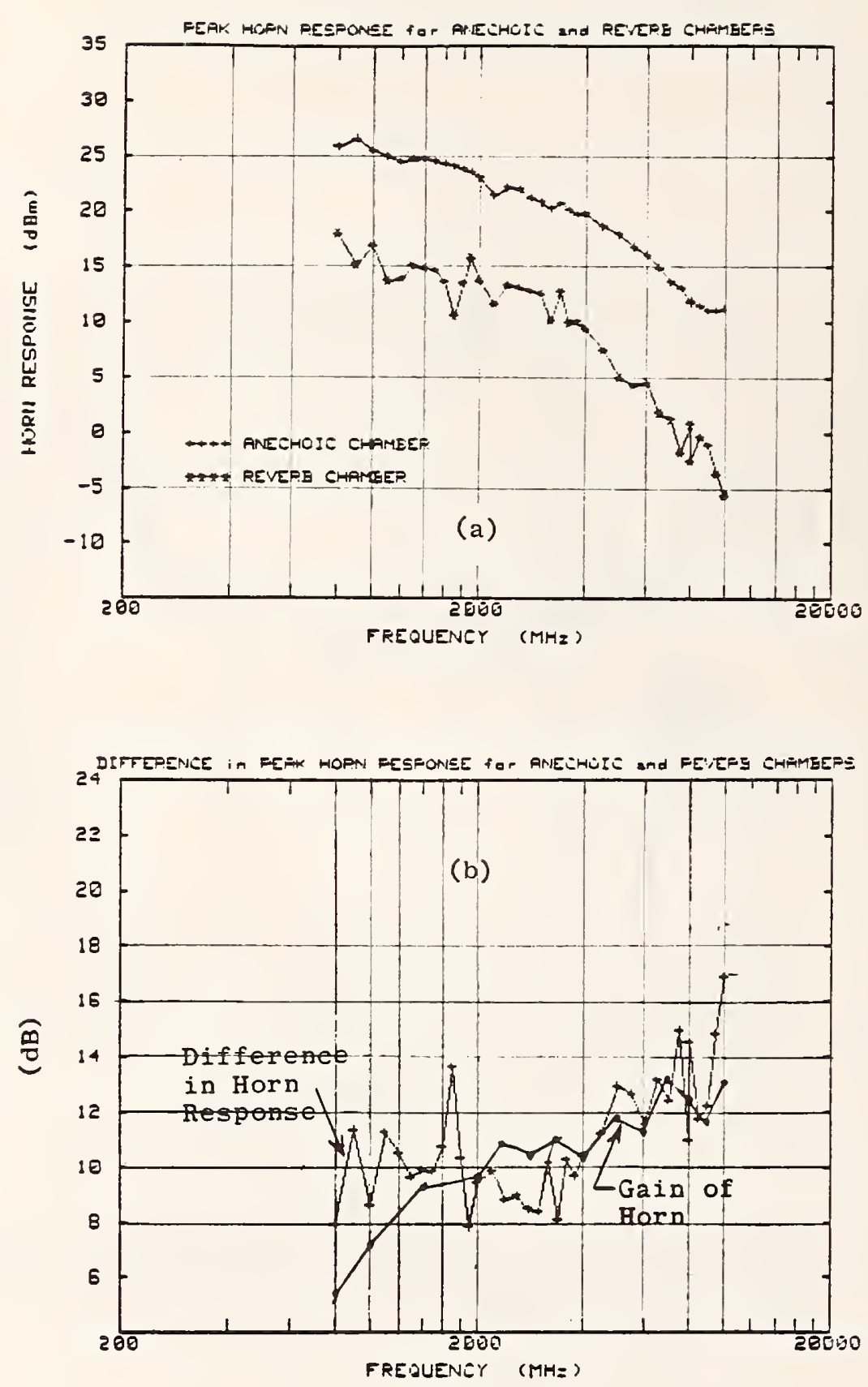

Figure 5.3 Comparison of ridged horn's peak responses to EM field established inside NBS reverberation and anechoic chambers. Output from horn normalized to exposure E-field of $37 \mathrm{~dB} \mathrm{~V} / \mathrm{m}$. a) Antenna output versus frequency, b) Difference in output responses of ridged horn measured in NBS reverberation and anecholc chambers compared to calibrated gain of horn. 


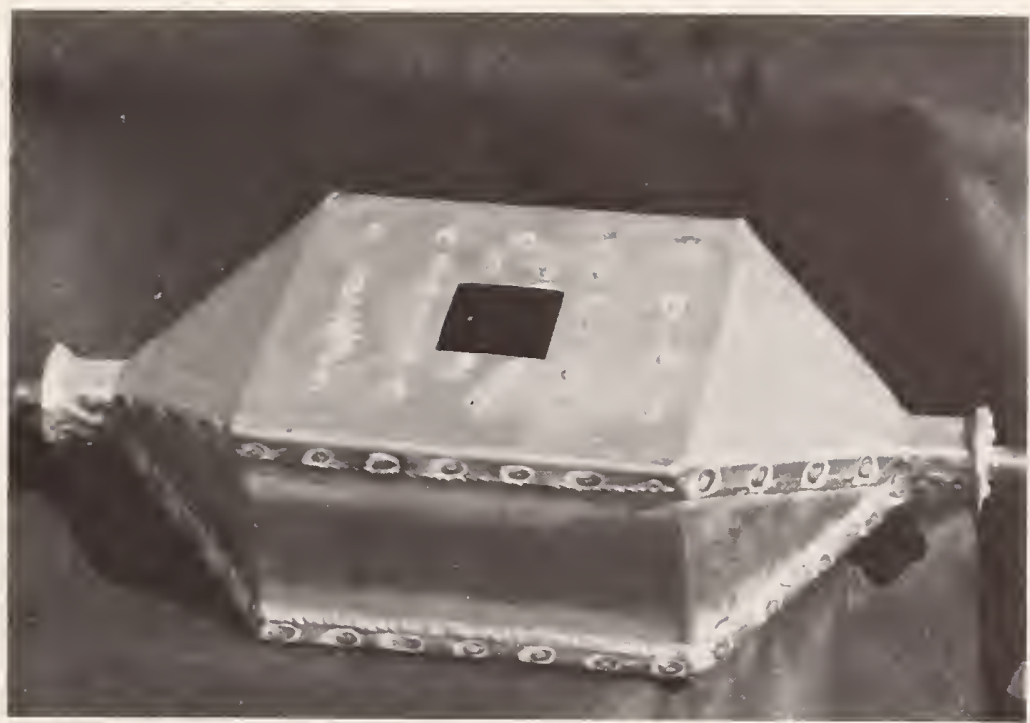

Figure 5.4 Photograph of $12 \mathrm{~cm} \times 18 \mathrm{~cm} \times 36 \mathrm{~cm}$ rectangular TEM cell with $5.1 \mathrm{~cm} \times 5.1 \mathrm{~cm}$ aperture.

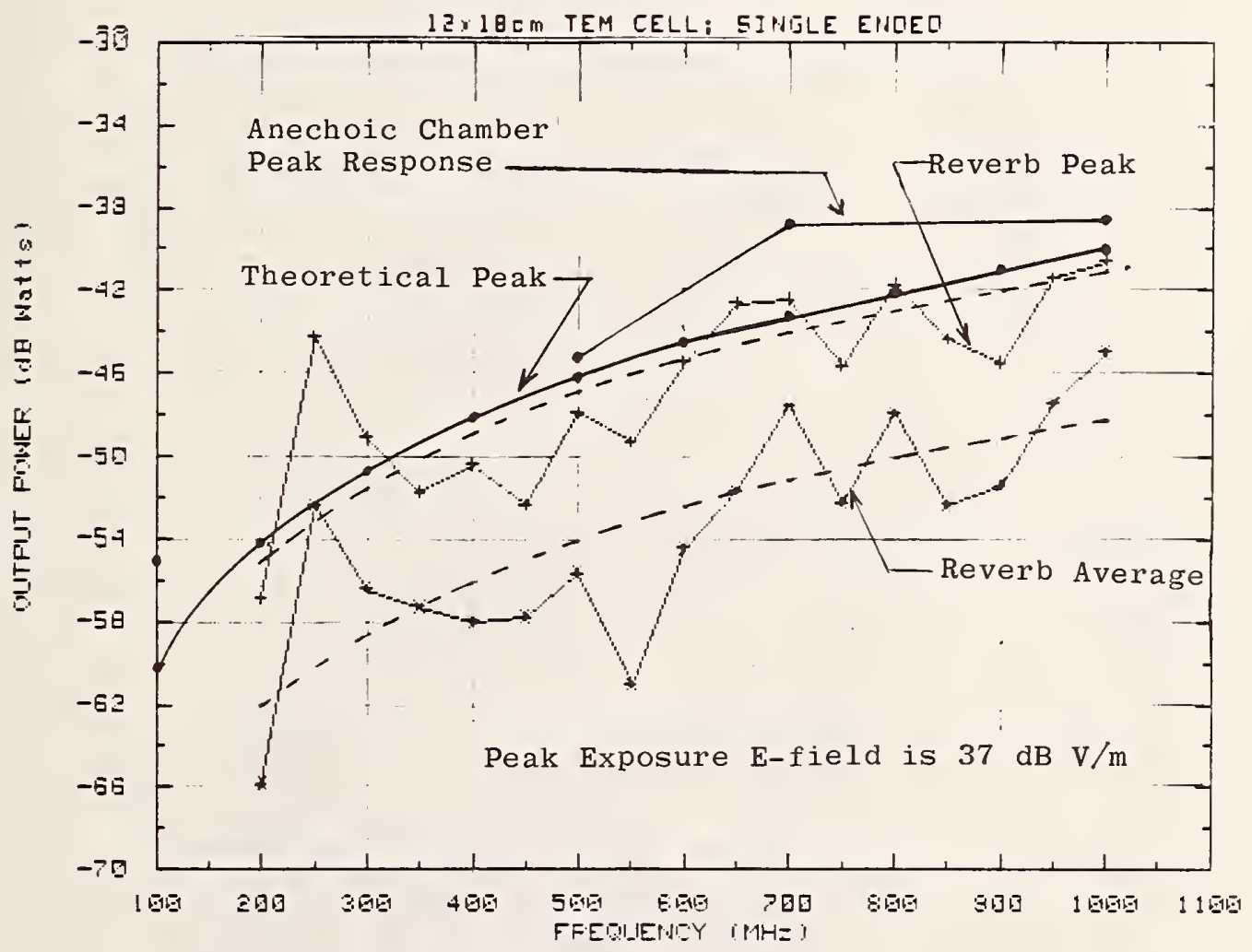

Figure 5.5 Comparison of power coupled to one port of $12 \mathrm{~cm} \times 18 \mathrm{~cm} \times 36$ $\mathrm{cm}$ TEM cell with $5.1 \mathrm{~cm} \times 5.1 \mathrm{~cm}$ aperture placed inside NBS reverberation and anechoic chambers. 


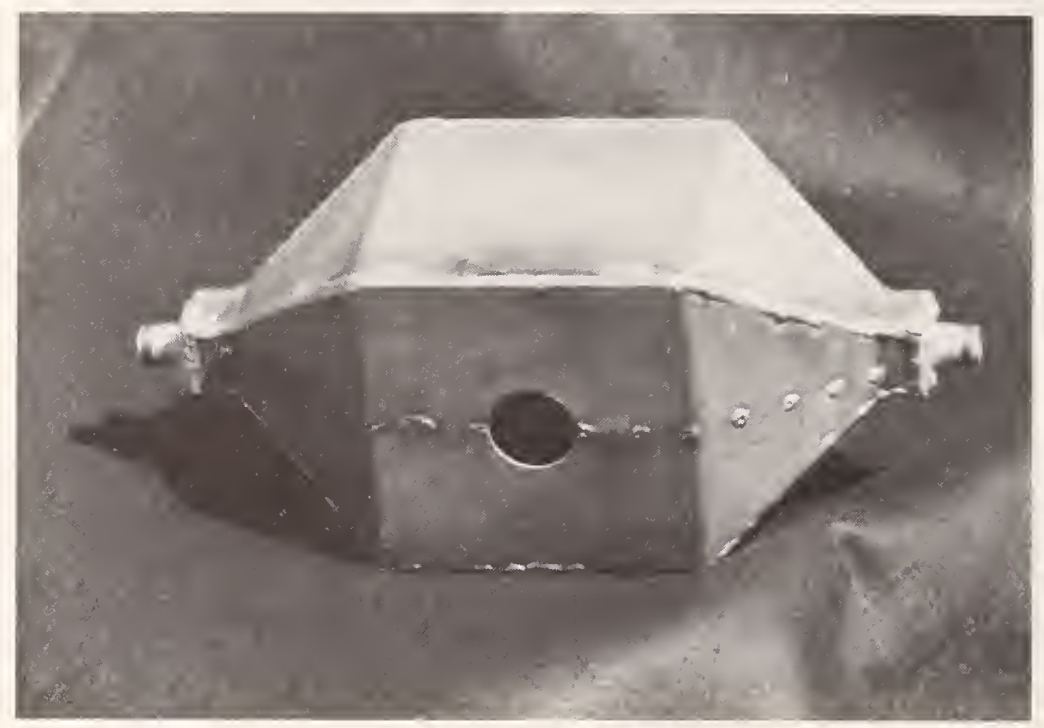

Figure 5.6 Photograph of $12 \mathrm{~cm} \times 12 \mathrm{~cm} \times 24 \mathrm{~cm}$ rectangular TEM cell with $3.1 \mathrm{~cm}$ diameter aperture.

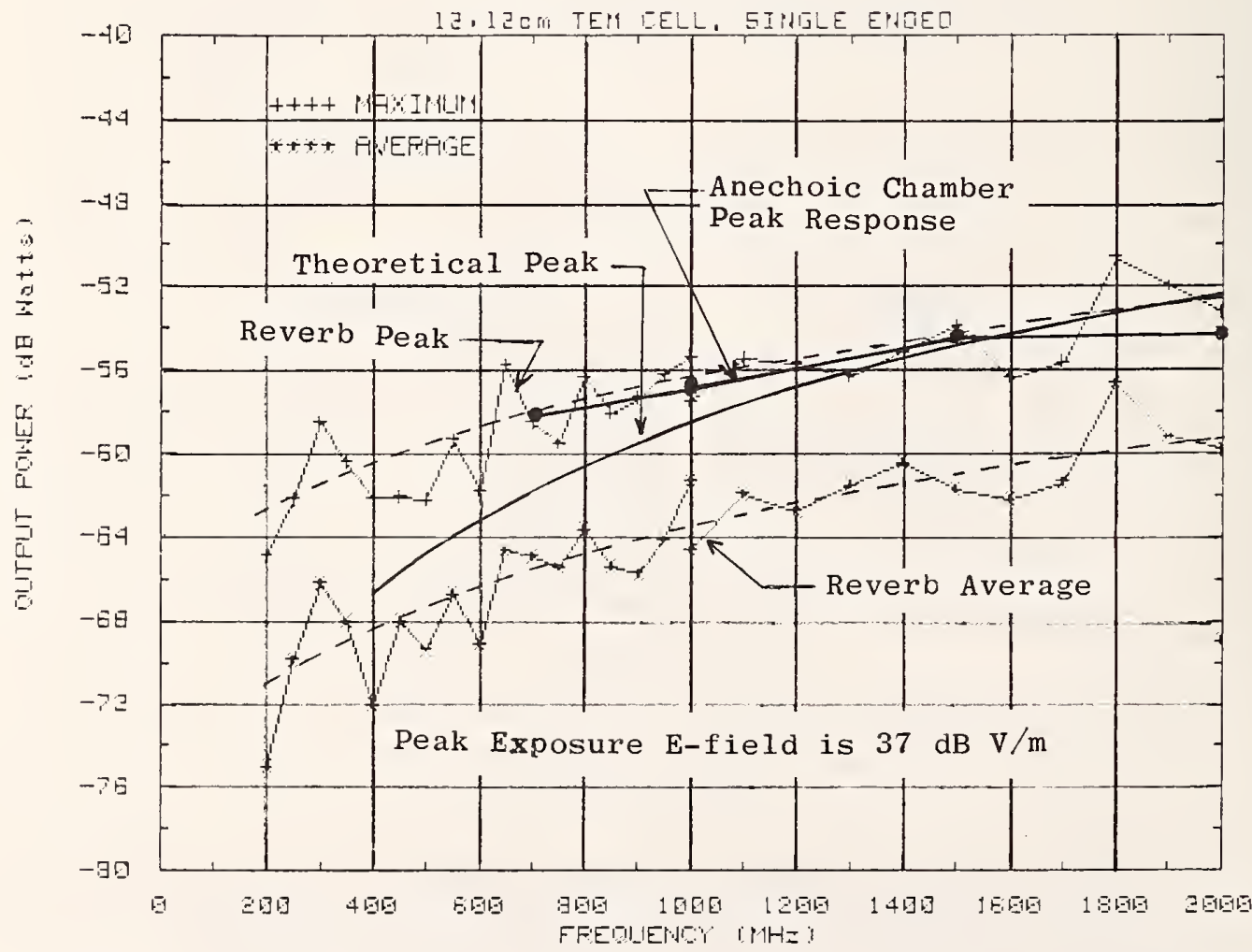

Figure 5.7 Comparison of power coupled to one port of $12 \mathrm{~cm} \times 12 \mathrm{~cm} \times 24$ $\mathrm{cm}$ TEM cell with $3.1 \mathrm{~cm}$ diameter aperture placed inside NBS reverberation and anechoic chambers. 


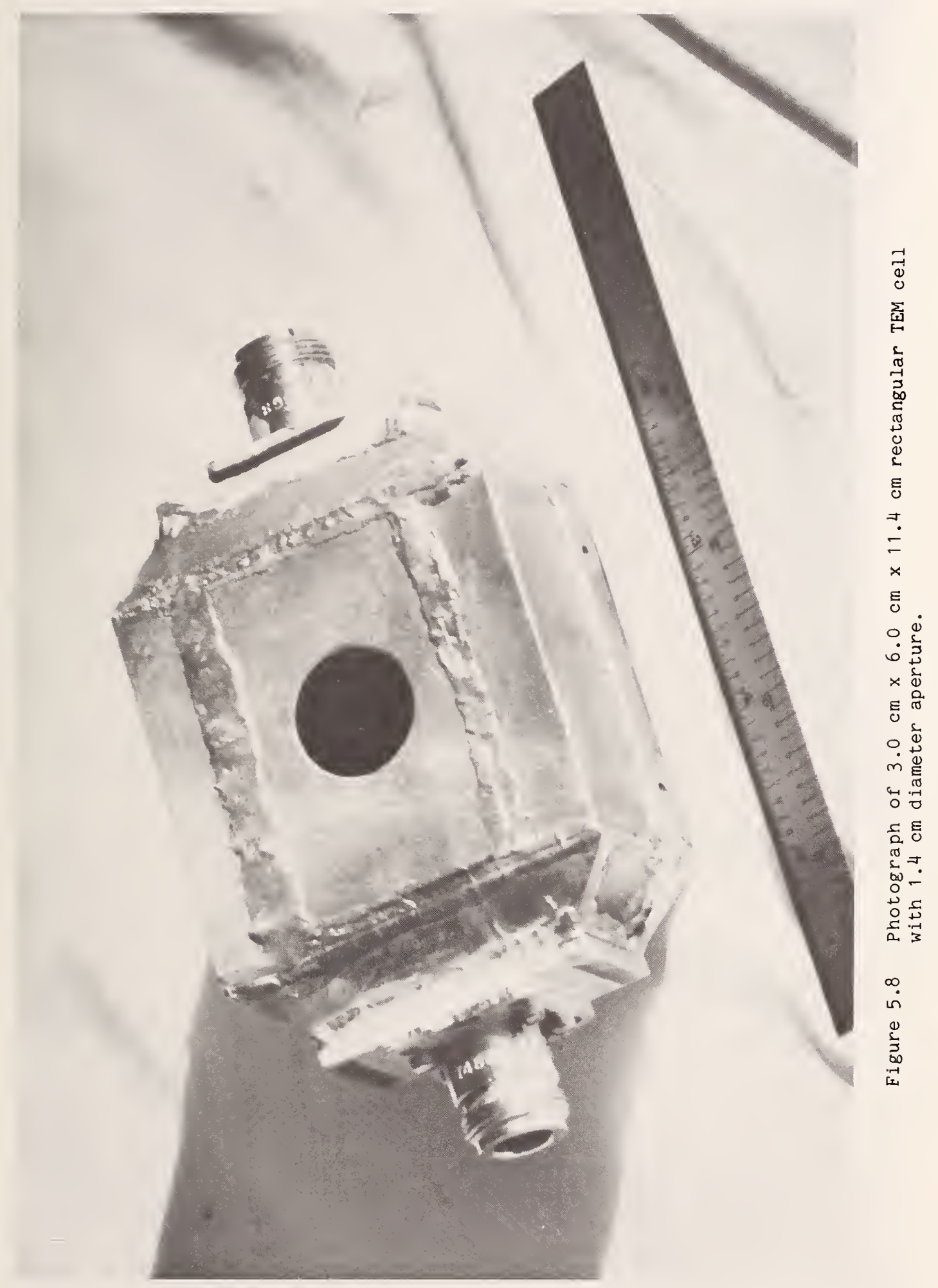




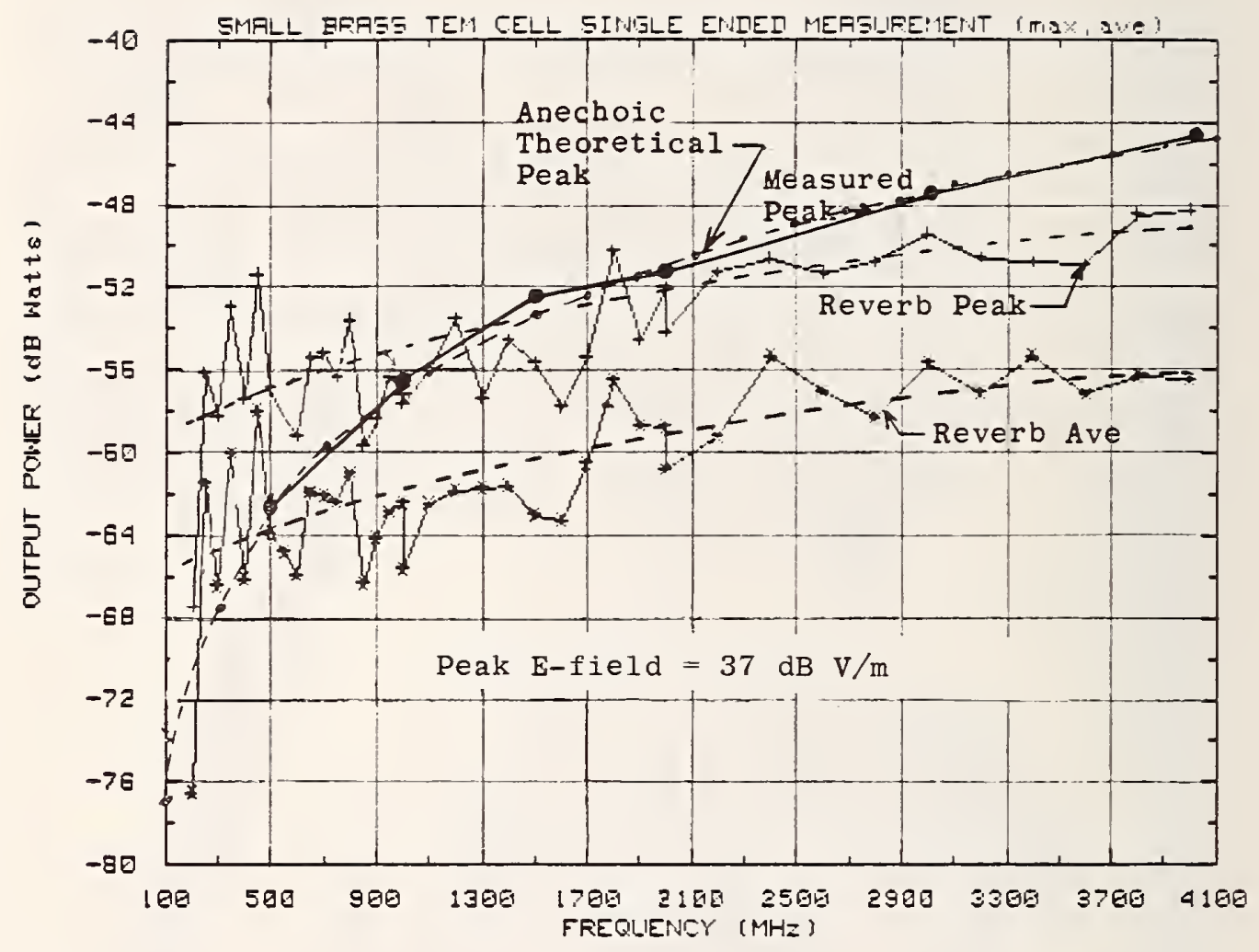

Figure 5.9 Comparison of power coupled to one port of $3.0 \mathrm{~cm} \times 6.0 \mathrm{~cm} \times$ $11.4 \mathrm{~cm}$ TEM cell with $1.4 \mathrm{~cm}$ diameter aperture placed inside NBS reverberation and anechoic chambers. 


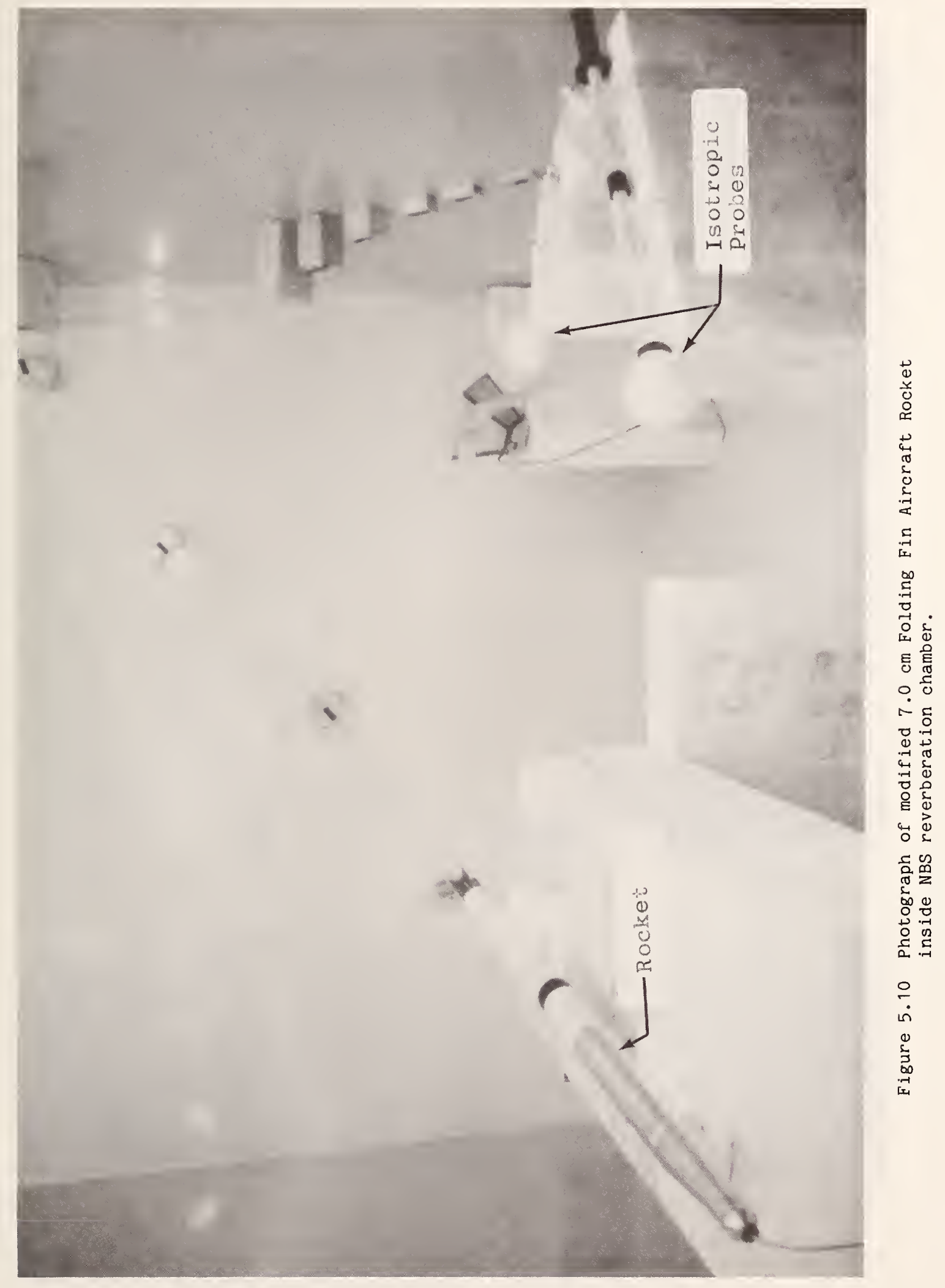




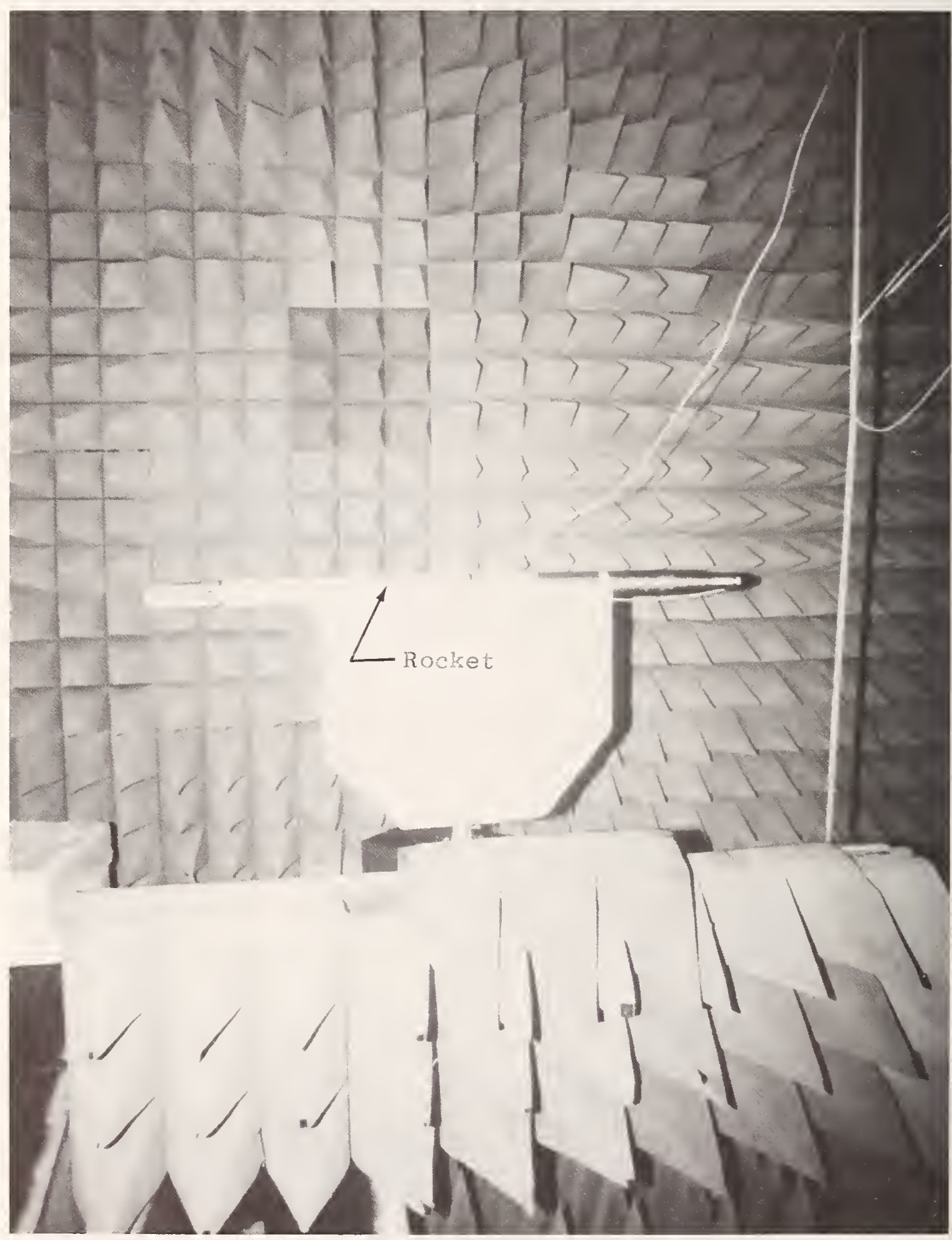

Figure 5.11 Photograph of modified $7.0 \mathrm{~cm}$ Folding Fin Aircraft Rocket inside NBS anechoic chamber. 

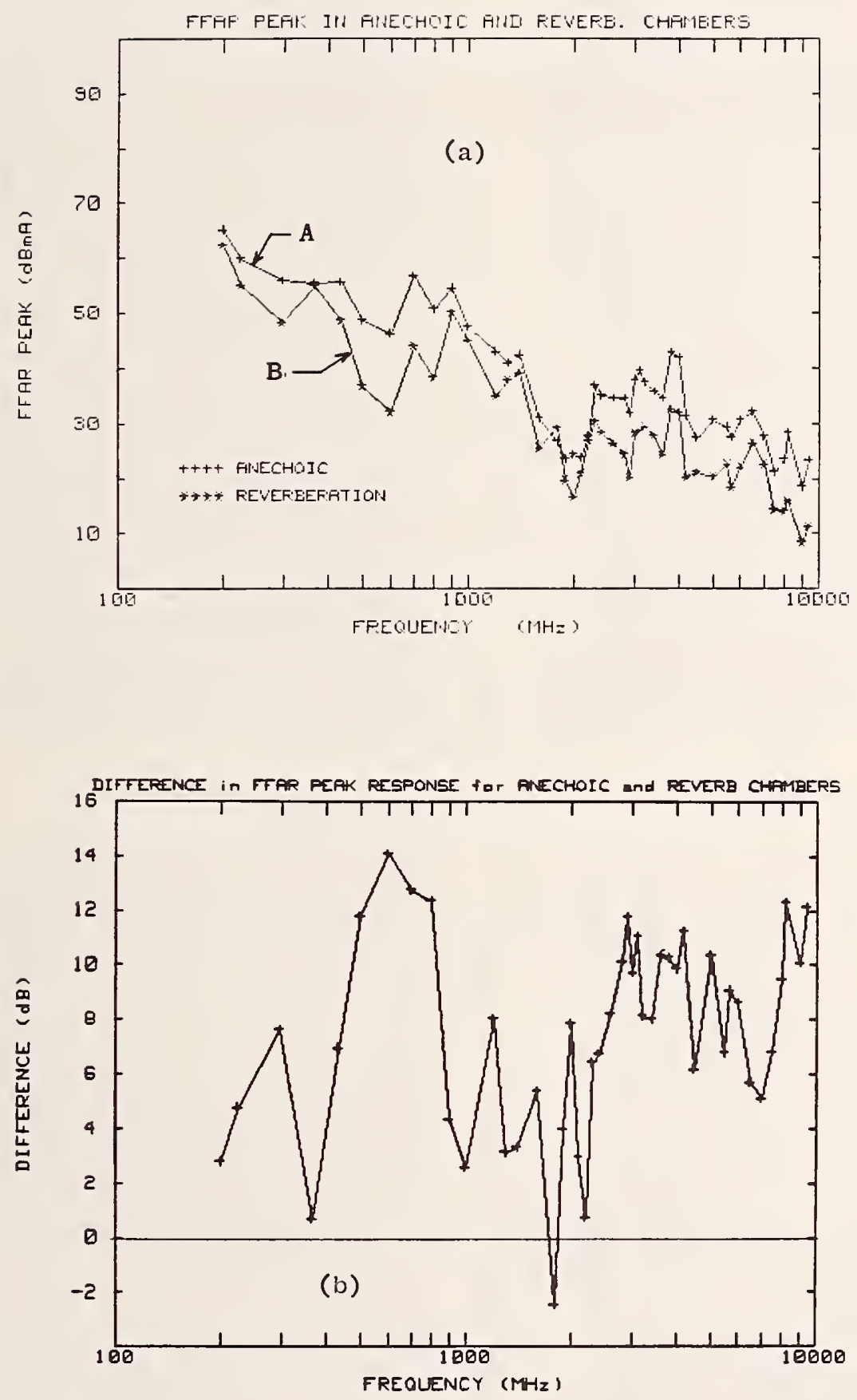

Figure 5.12 Comparison of $7.0 \mathrm{~cm}$ modified FFAR thermocouple responses to EM field established inside NBS reverberation and anechoic chambers. Data normalized to exposure power density of 10 $\mathrm{mw} / \mathrm{cm}^{2}$. a) Thermocouple output vs frequency. b) Difference in thermocouple output measured in NBS reverberation and anechoic chambers. 

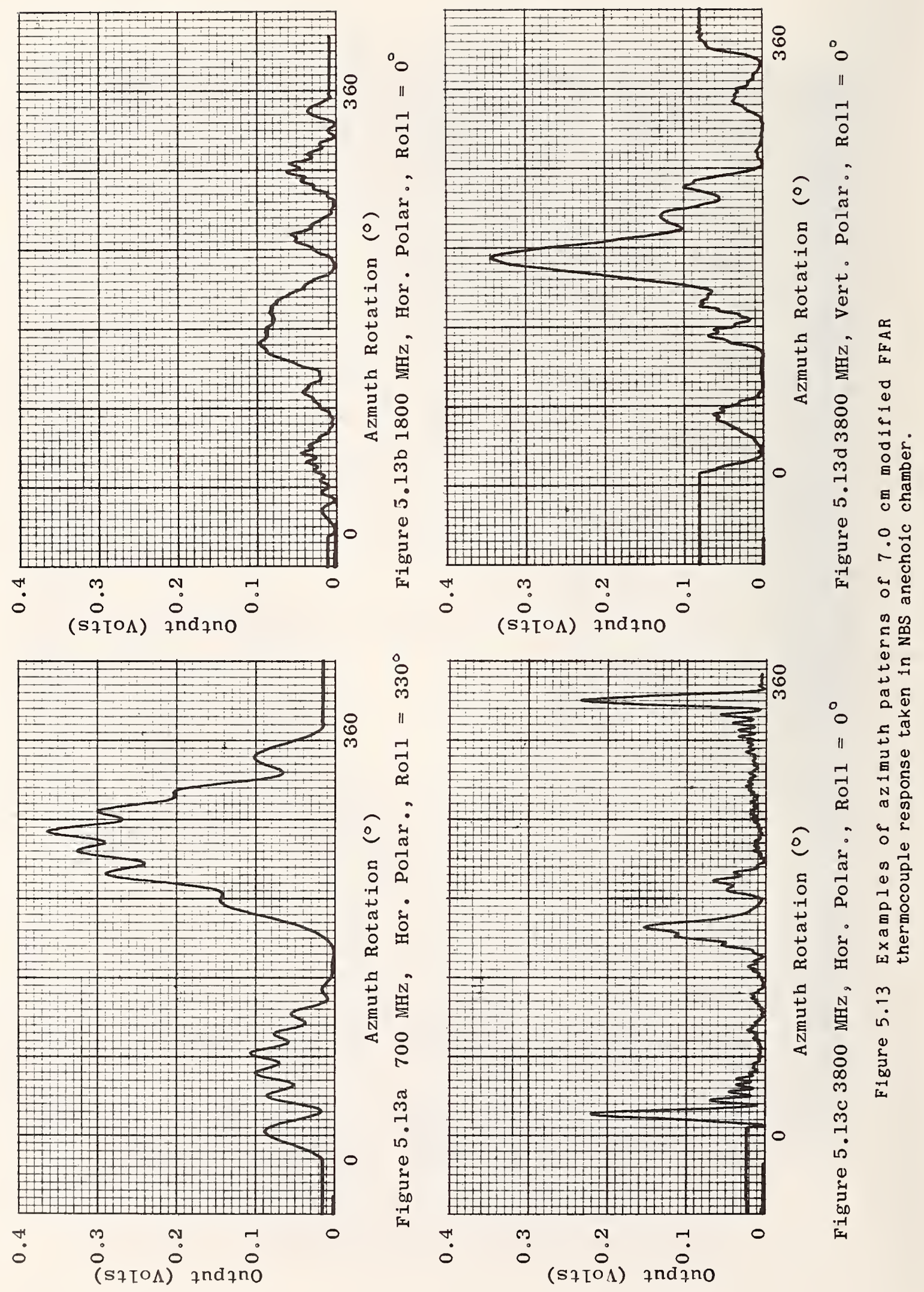


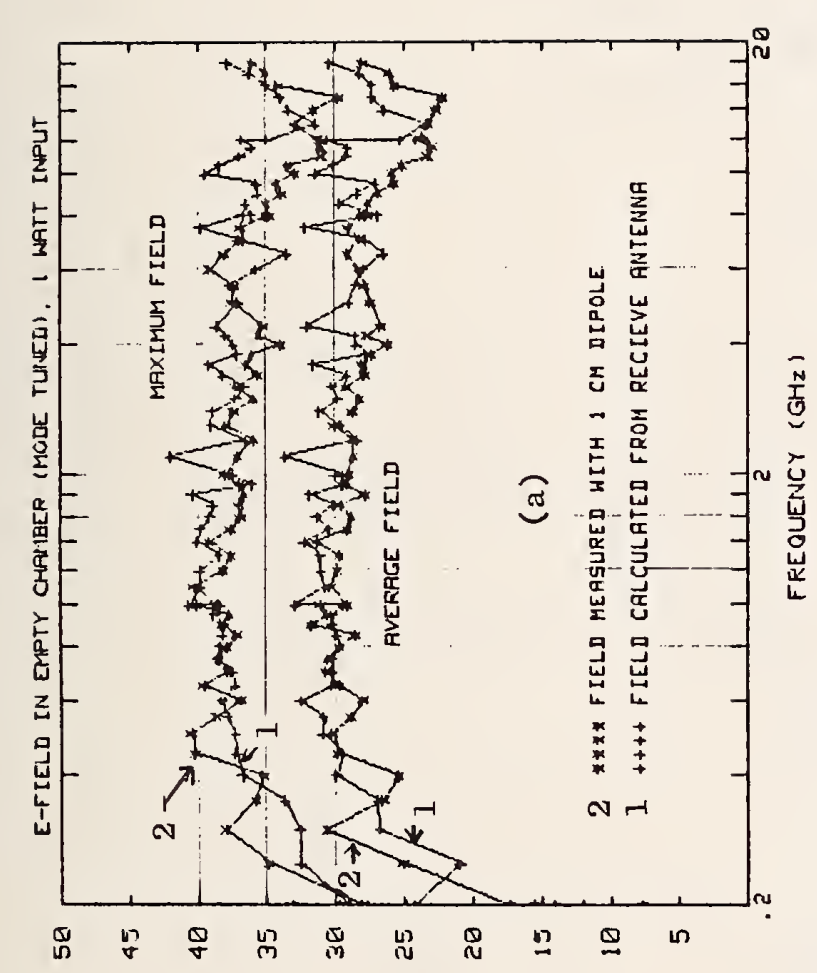

(w/A gp) ด ם

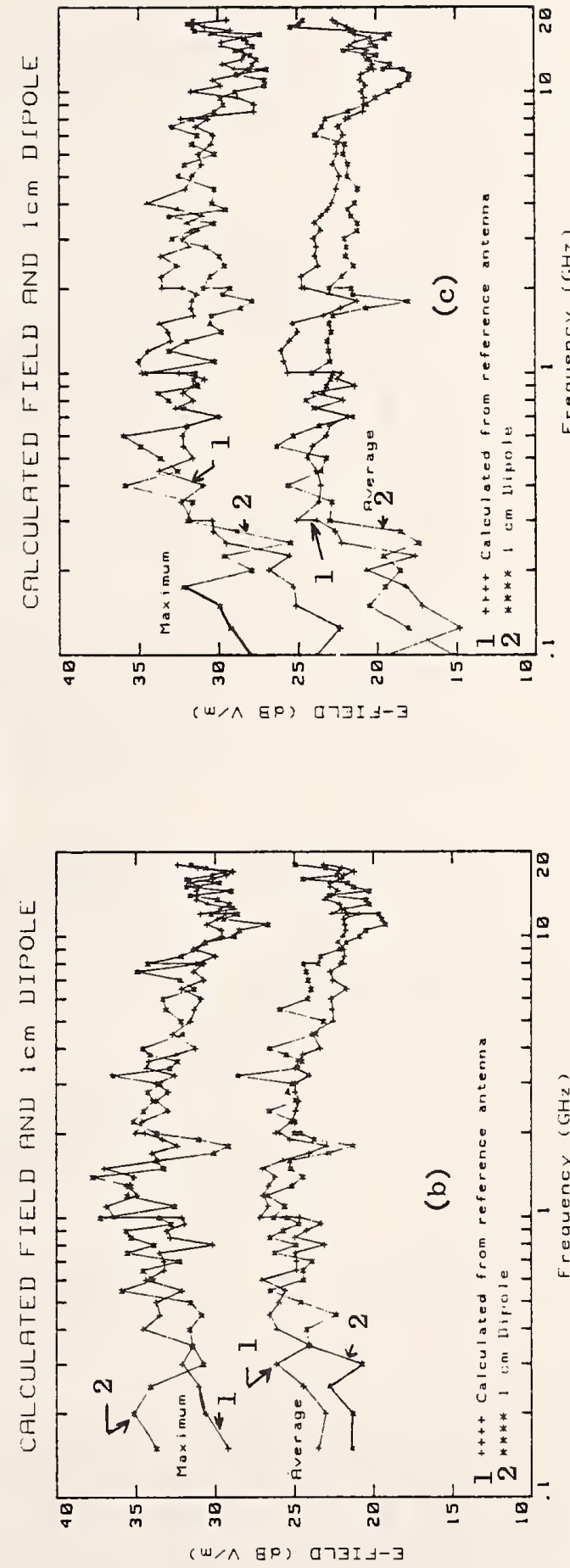

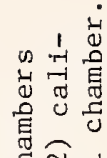

离 จิ

돈

त्र

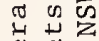

$\stackrel{0}{0}$

过苞已

足

( $\pi$

药

乙

范范夏

क व

z: 先

I

(4)

(⿻)

단 ब

पू

氖易

记

두

o 2

फ्न त्न

a

E के के

更

远是

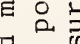

䒜山元

ब 呈

कo

总步

$\sum_{0}^{0}$

व

+4

40 .

등 명

क E

㝳是

号岕贯

苛

0
4
0
0
-1
$x$ 


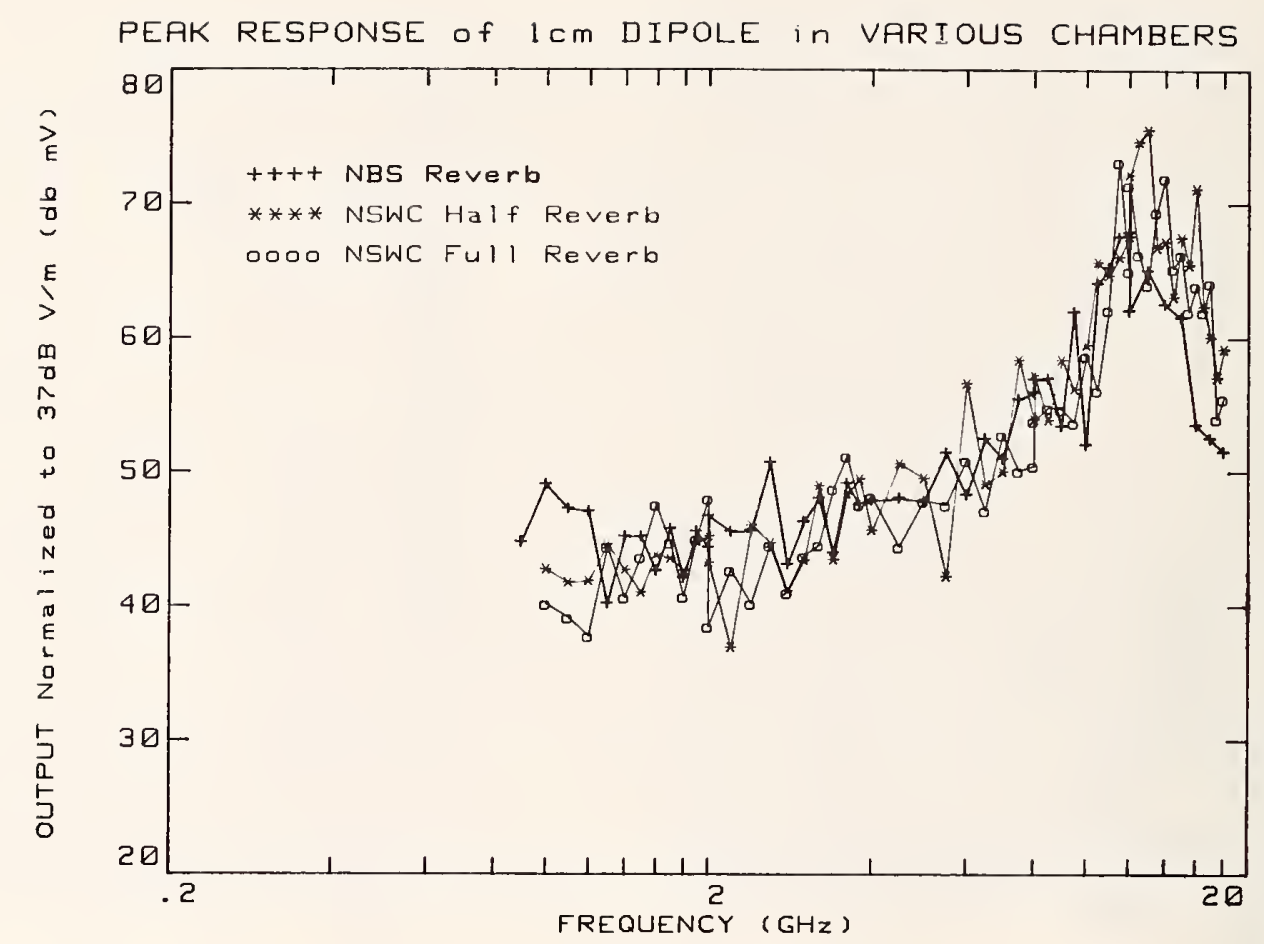

Figure 5.15 Comparison of $1 \mathrm{~cm}$ dipole probe's peak response to normalized E-field of $37 \mathrm{~dB} \mathrm{V/m}$ using NBS and NSWC reverberation chambers.

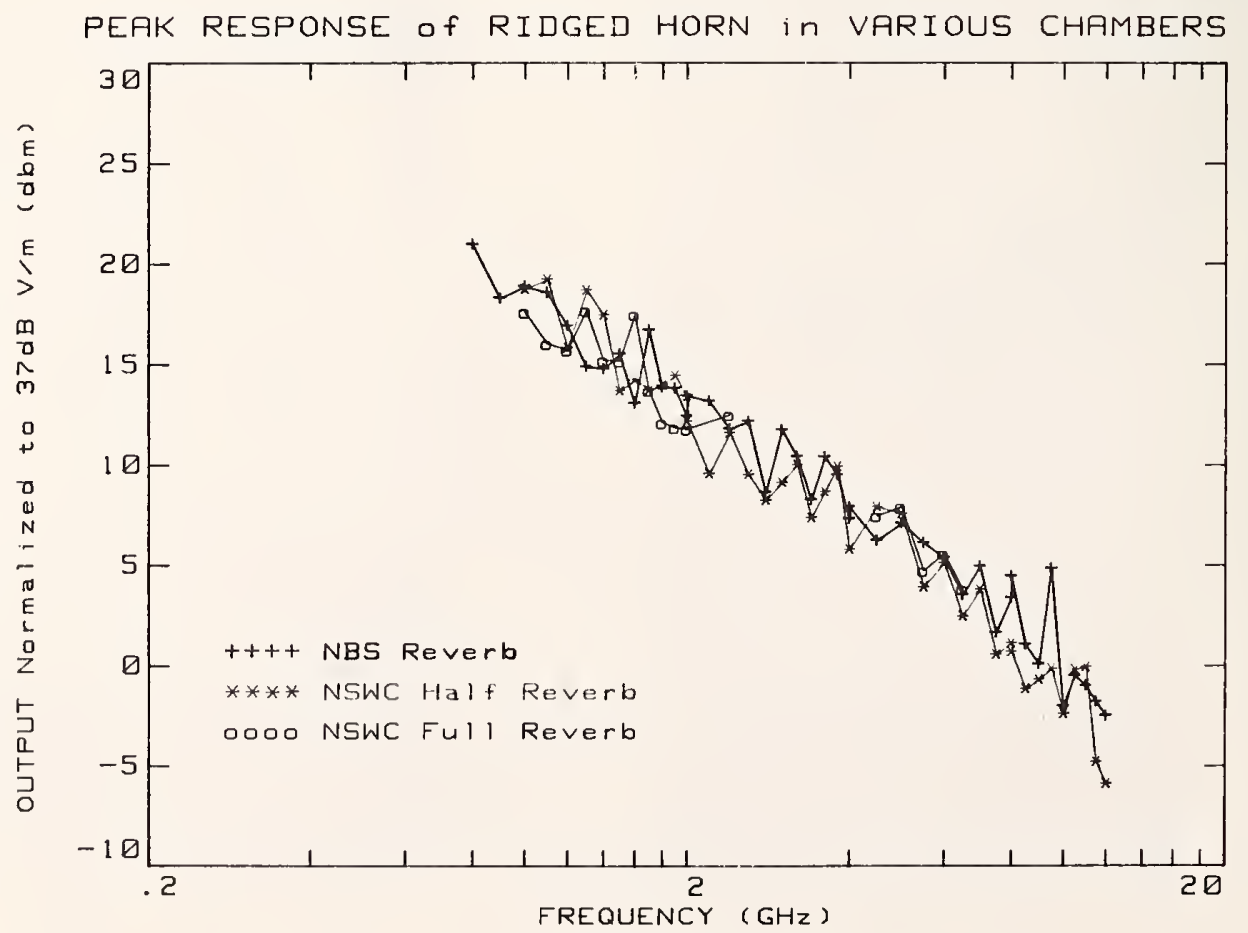

Figure 5.16 Comparison of ridged horn's peak responses to normalized exposure E-field of $37 \mathrm{~dB} \mathrm{~V} / \mathrm{m}$ using NBS and NSWC reverberation chambers. 

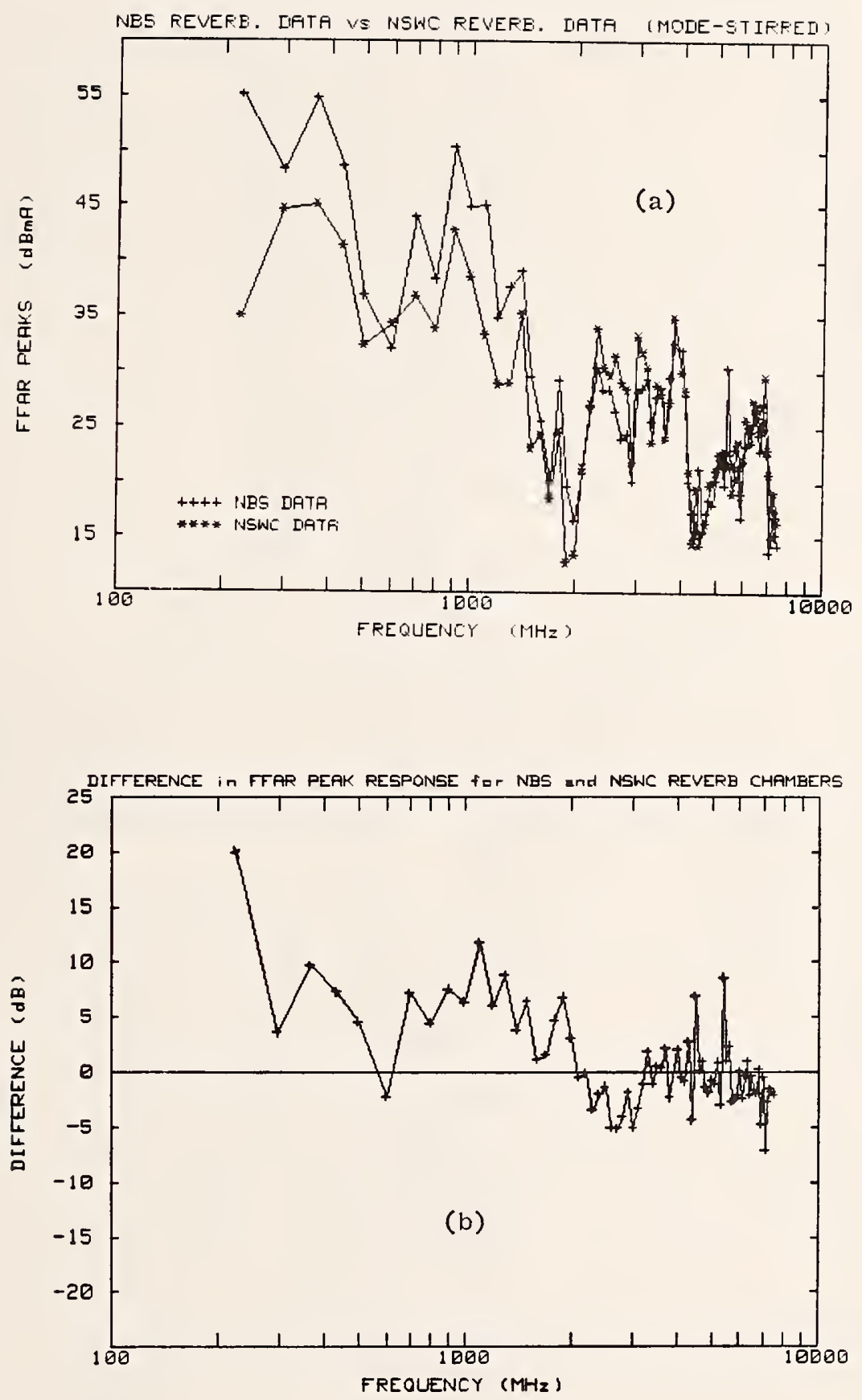

Figure 5.17 Comparison of $7.0 \mathrm{~cm}$ modified FFAR thermocouple responses to EM field established inside NBS and NSWC half reverberation chambers. Data normalized to exposure power density of 10 $\mathrm{mW} / \mathrm{cm}^{2}$. a) Thermocouple output vs frequency. b) Difference in thermocouple output measured in NBS and NSWC reverberation chambers. 

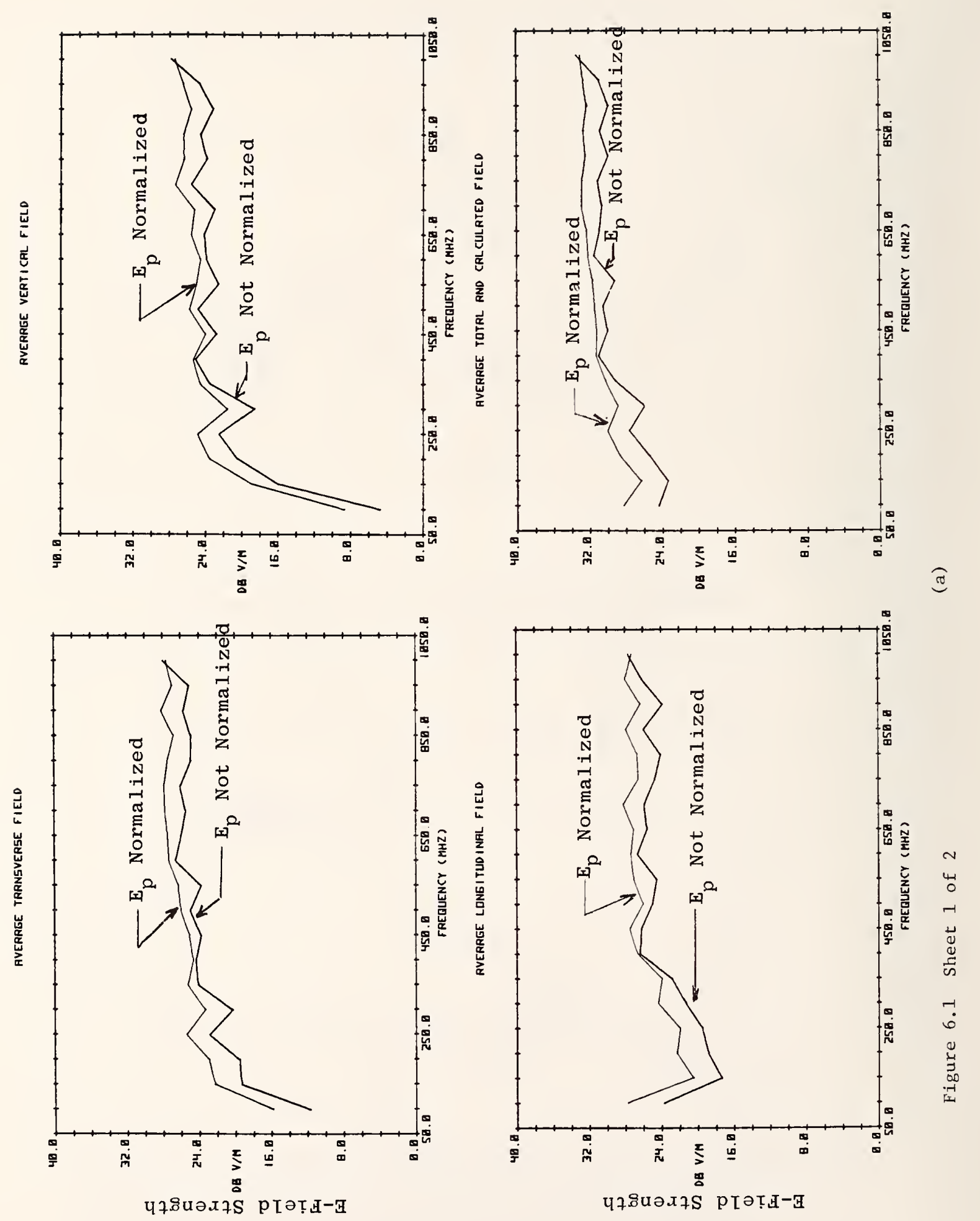

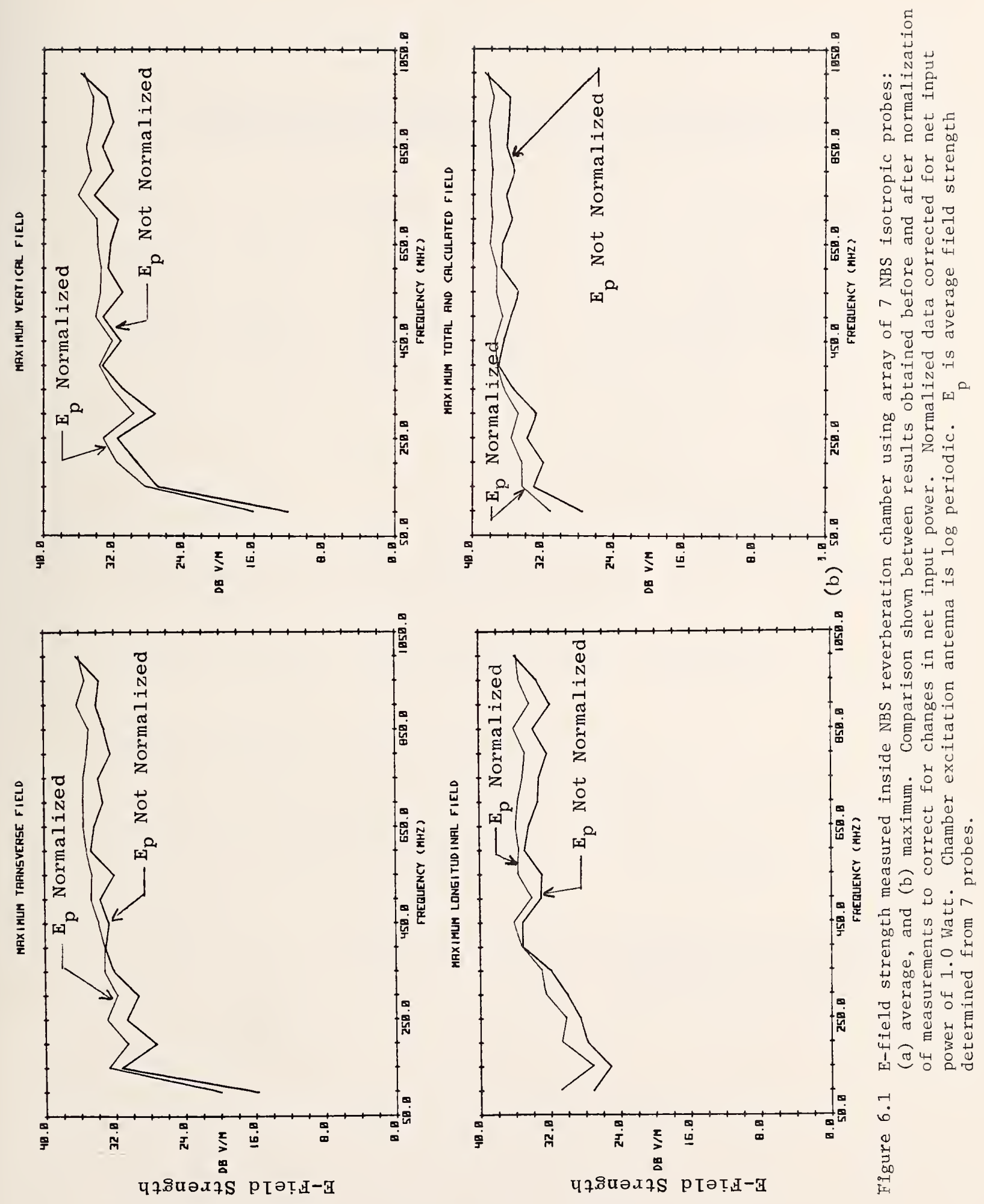
Table 6.1 Summary and estimates of measurement uncertainties for determining field strength inside NBS reverberation chamber Mode Tuned (200 $\mathrm{MHz}-2.0 \mathrm{GHz}$ )

\section{Source of Error}

1) Measuring Received Power Cable Loss Attenuator Calibration

Antenna Efficiency Power Meter Calibration Total

2) Receiving Power Mismatch (See Figure 6.1)

3) Mixing/Sampling Efficiency Spatial Field Uniformity (see figure 2.27) Limited Sample Size (see Table 6.4)

Total

4) Wave Impedance $\neq 120 \pi$ (see Figure 2.25)

Total Worst Case Error

RSS Error

5) Failure to Correct for Input Power Variations (Transmit Mismatch Error)

\section{Error $(\mathrm{dB})$}

$200 \mathrm{MHz} \quad 500 \mathrm{MHz} \quad 1.0 \mathrm{GHz} \quad 2.0 \mathrm{GHz}$ Ave. Max. Ave. Max. Ave. Max. Ave. Max.

$$
\begin{array}{llll} 
\pm 0.05 & \pm 0.05 & \pm 0.05 & \pm 0.10 \\
\pm 0.10 & \pm 0.10 & \pm 0.10 & \pm 0.10 \\
\pm 0.05 & \pm 0.05 & \pm 0.05 & \pm 0.10 \\
\pm 0.20 & \pm 0.20 & \pm 0.20 & \pm 0.20 \\
\cline { 2 - 2 } & \pm 0.40 & \pm 0.40 & \pm 0.50
\end{array}
$$

$$
\begin{array}{llllllll}
-2.8 & -5.1 & -1.5 & -3.4 & -0.7 & -1.5 & -0.3 & -0.7
\end{array}
$$

$$
\begin{array}{cccc} 
\pm 8.0 & \pm 5.0 & \pm 3.0 & \pm 2.0 \\
\pm 0.2 \pm 0.5 & \pm 0.2 \pm 0.5 & \pm 0.2 \pm 1.5 & \pm 0.3 \pm 1.0 \\
\hline \pm 8.2 \pm 8.5 & \pm 5.2 \pm 5.5 & \pm 3.2 \pm 4.5 & \pm 2.3 \pm 3.0
\end{array}
$$

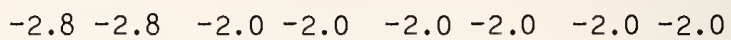
$+2.0+6.0+2.0+6.0+2.0+4.5+2.0+3.0$

$-1 \overline{4.2-16.8} \overline{-9.1-11.3} \overline{-6.3-8.4} \overline{-5.1-6.2}$ $+10.6+14.9+7.6+11.9+5.6+9.4+4.8+6.5$

$-\overline{-9.1-10.3} \overline{-5.8-6.8} \overline{-3.9-5.2} \overline{-3.1-3.7}$
$+8.5+10.4+5.6+8.1+3.8+6.4+3.1+4.3$

$\begin{array}{llll}-4.0 & -3.0 & -2.0 & -1.0\end{array}$


Table 6.2 Summary and estimates of measurement uncertainties for
determining field strength inside NBS reverberation chamber Mode Stirred $(2.0 \mathrm{GHz}-18.0 \mathrm{GHz})$

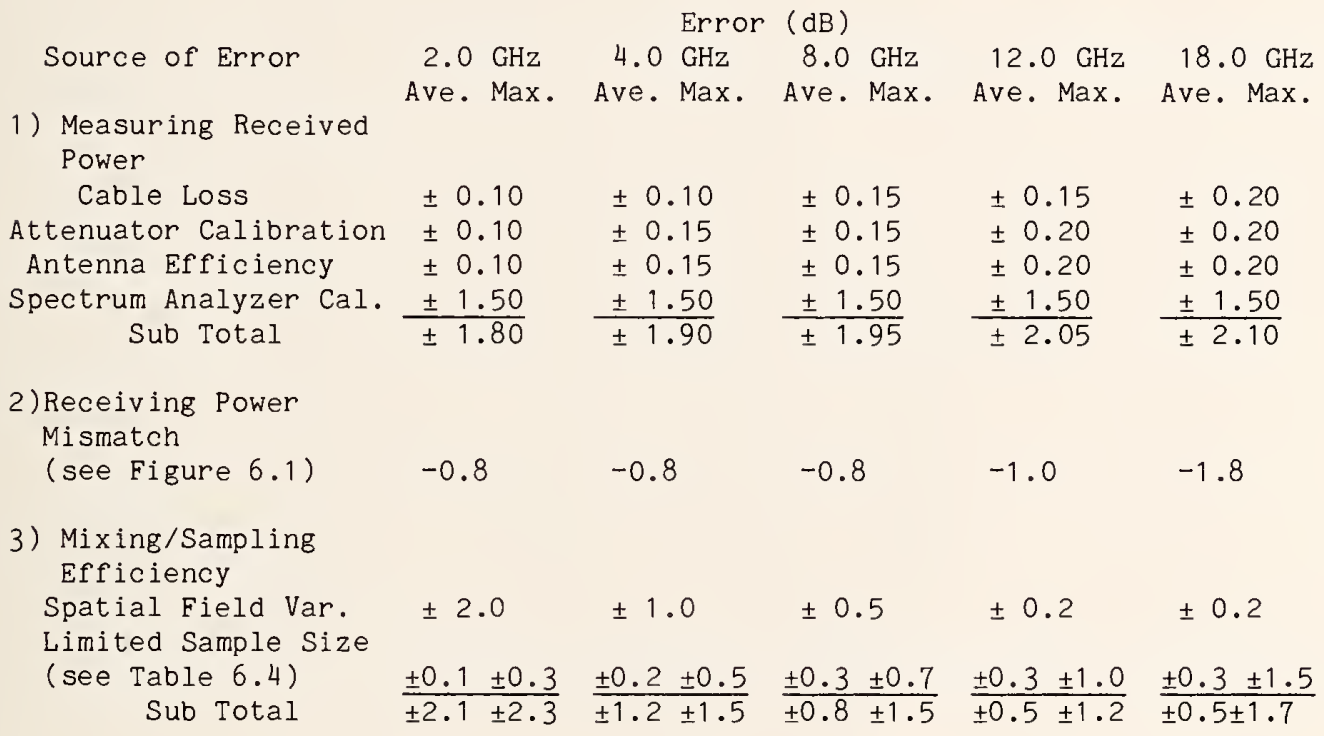

4) Wave Imped $\neq 120 \pi \quad$ Average $\leqq \pm 2.0,-2.0 \geqq$ Maximum $\leqq+3.0$

5) Failure to Correct for Input Power Var.

$-0.8$

$-0.8$

$-1.0$

$-1.8$

$\begin{array}{rlrlllll}\text { Total(Worst Case) Error }-7.5-7.7 & -6.7-7.0 & -6.4 & -6.8 & -6.6 & -7.3 & -8.2 & -9.4 \\ & +5.9+7.1 & +5.1+6.4 & +4.8+6.2 & +4.6 & +6.3 & +4.6 & +6.8\end{array}$

RSS Error

$-3.6-3.7-3.1-3.3-3.1-3.2-3.2-3.4-3.9-4.2$
$+3.4+4.2+3.0+3.9+2.9+3.8+2.9+3.8+2.9+4.0$ 
Table 6.3 Estimates of impedance mismatch uncertainties for received power measurements

\begin{tabular}{cccccc}
$\begin{array}{c}\text { Frequency } \\
\text { GHz }\end{array}$ & \multicolumn{3}{c}{ Source VSWR } & Load & \multicolumn{2}{c}{ Mismatch Error } \\
0.2 & 5.0 & 10.0 & 1.10 & -2.83 & -5.15 \\
0.5 & 3.0 & 6.0 & 1.10 & -1.46 & -3.40 \\
1.0 & 2.0 & 3.0 & 1.10 & -0.66 & -1.46 \\
2.0 & 1.5 & 2.0 & 1.10 & -0.27 & -0.66 \\
2.0 & 2.0 & 2.0 & 1.20 & -0.81 & -0.81 \\
4.0 & 2.0 & 2.0 & 1.20 & -0.81 & -0.81 \\
8.0 & 2.0 & 2.0 & 1.20 & -0.81 & -0.81 \\
12.0 & 2.0 & 2.0 & 1.30 & -0.95 & -0.95 \\
18.0 & 2.5 & 2.5 & 1.50 & -1.77 & -1.77
\end{tabular}

Table 6.4 Estimates of uncertainties due to limiting number of tuner positions (sample size).

\begin{tabular}{|c|c|c|c|c|c|}
\hline \multirow{3}{*}{$\begin{array}{l}\text { Frequency } \\
\text { GHz }\end{array}$} & \multicolumn{5}{|c|}{$\begin{array}{c}\text { Error due to limiting Sample Size } \\
\text { Ave/Max (dB) }\end{array}$} \\
\hline & & Number & Tuner Po & ions & \\
\hline & 50 & 100 & 200 & 400 & 800 \\
\hline 0.2 & $0.2 / 0.8$ & $0.2 / 0.5$ & -- & -- & -- \\
\hline 0.5 & $0.6 / 3.6$ & $0.5 / 2.0$ & $0.2 / 0.5$ & -- & -- \\
\hline 1.0 & -- & $1.0 / 4.0$ & $0.2 / 1.5$ & $0.2 / 0.5$ & $0.1 / 0.2$ \\
\hline 2.0 & -- & -- & $0.5 / 3.0$ & $0.3 / 1.0$ & $0.1 / 0.5$ \\
\hline 4.0 & -- & -- & -- & $0.5 / 3.0$ & $0.2 / 1.0$ \\
\hline 8.0 & -- & -- & -- & -- & $0.5 / 3.0$ \\
\hline
\end{tabular}


APPENDIX

Computer Programs for

Reverberation Chamber Immunity Measurements 


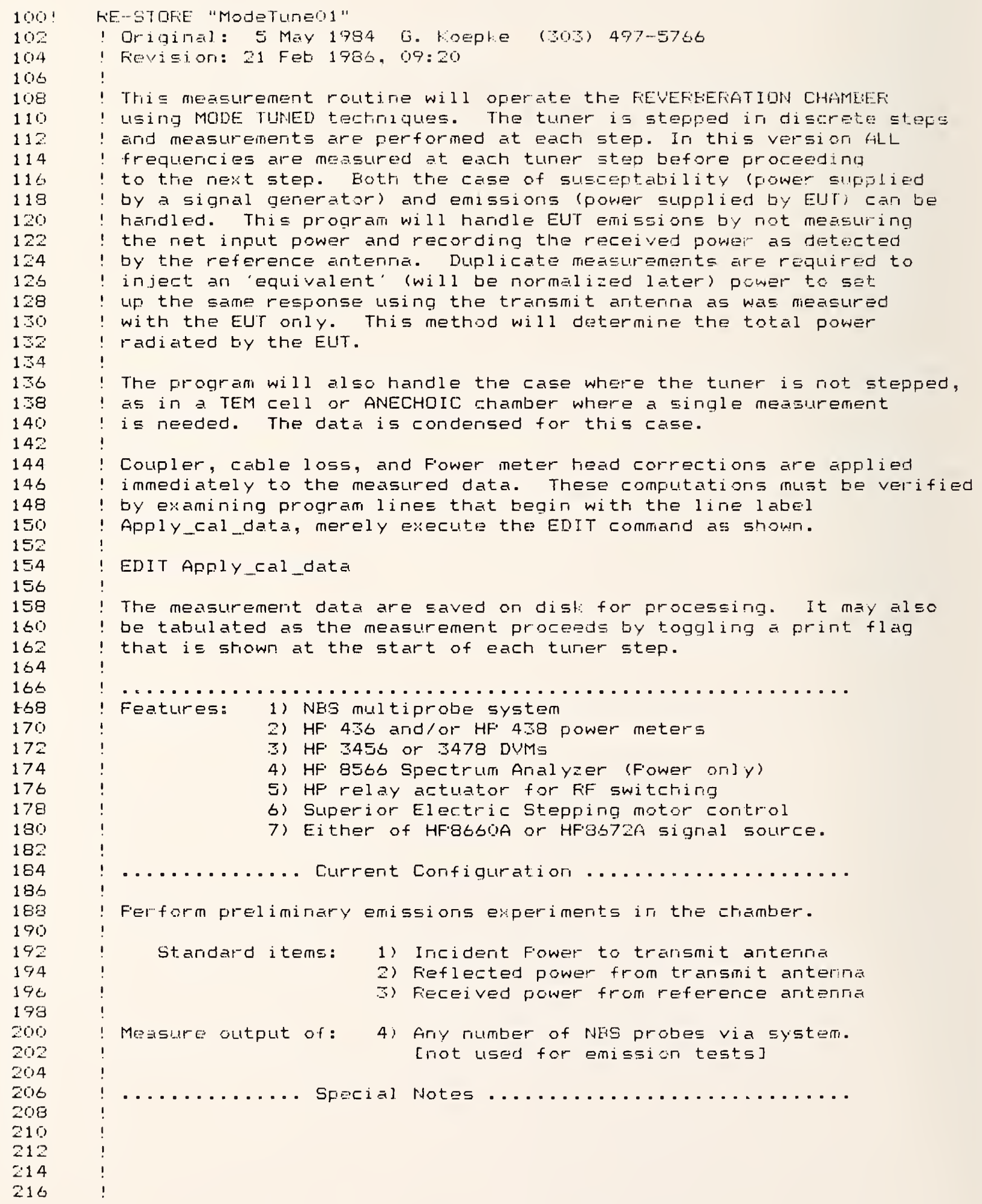




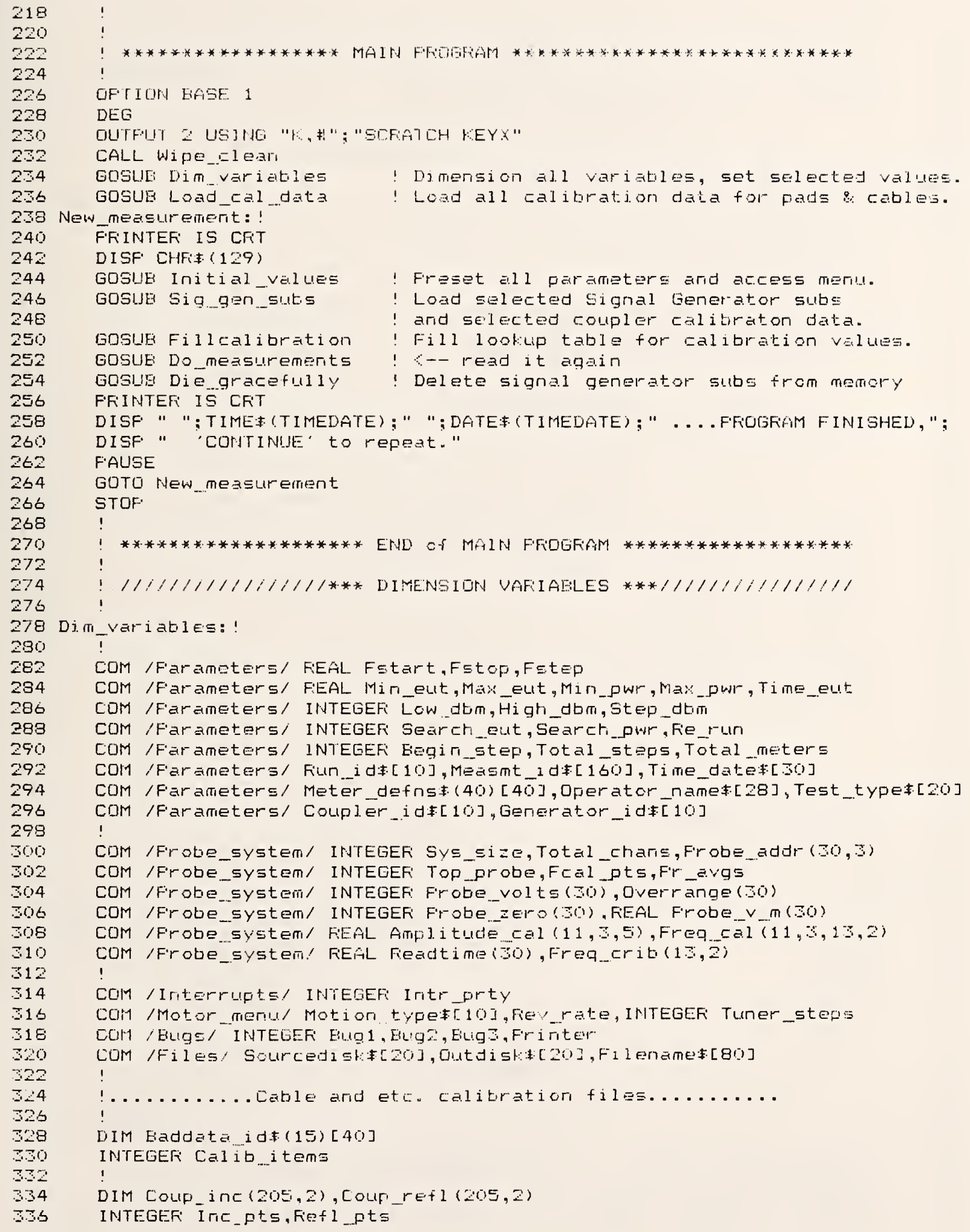




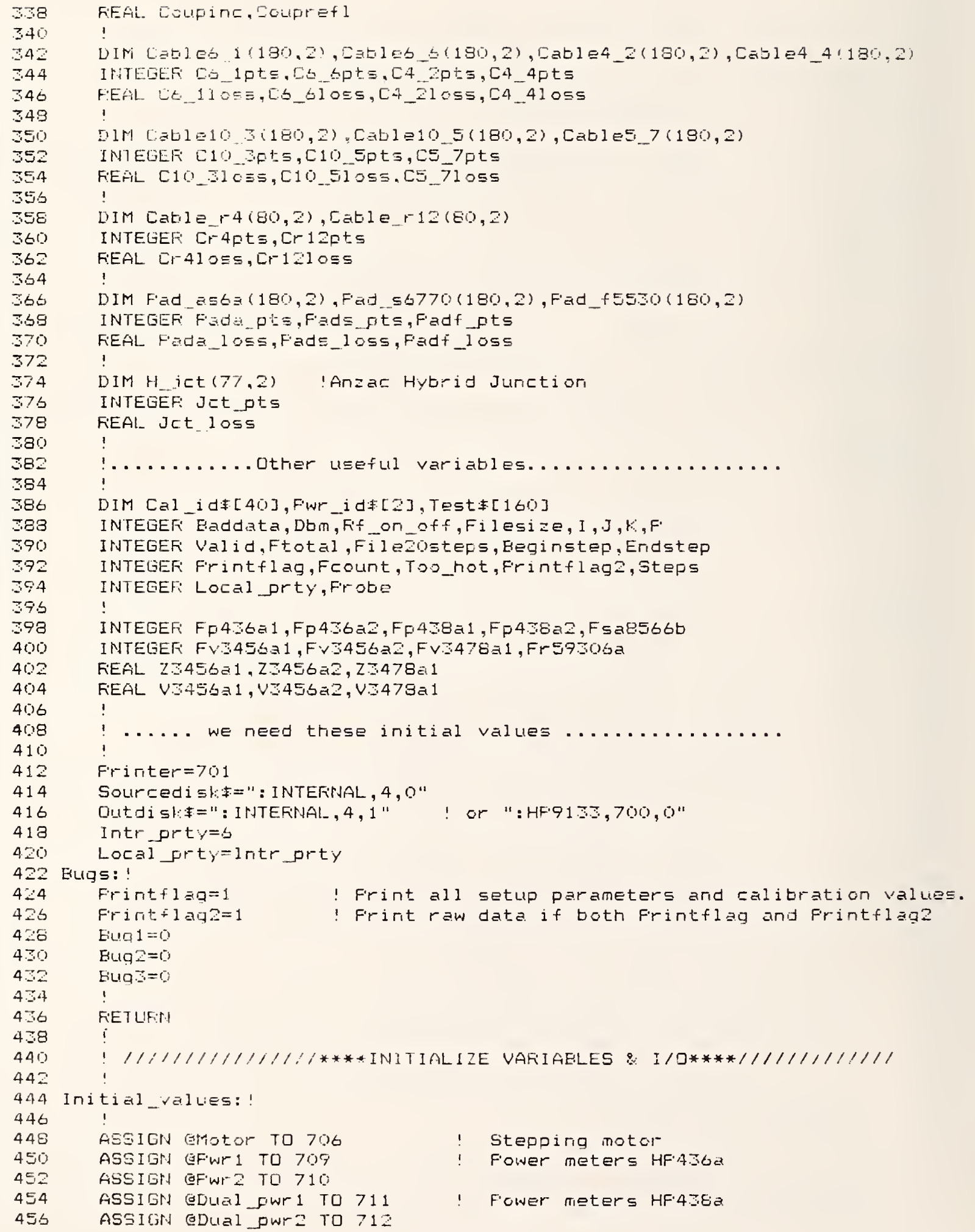




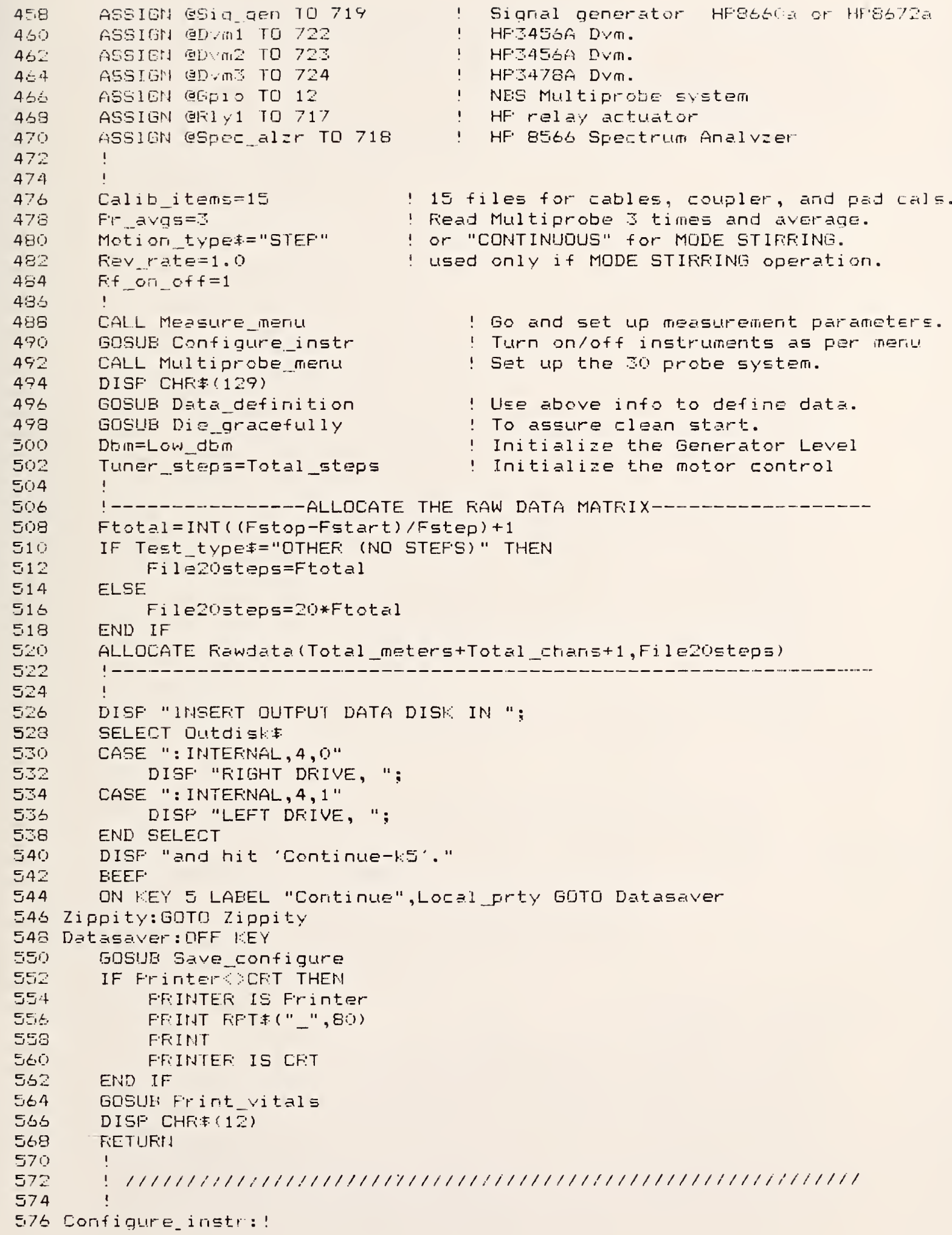




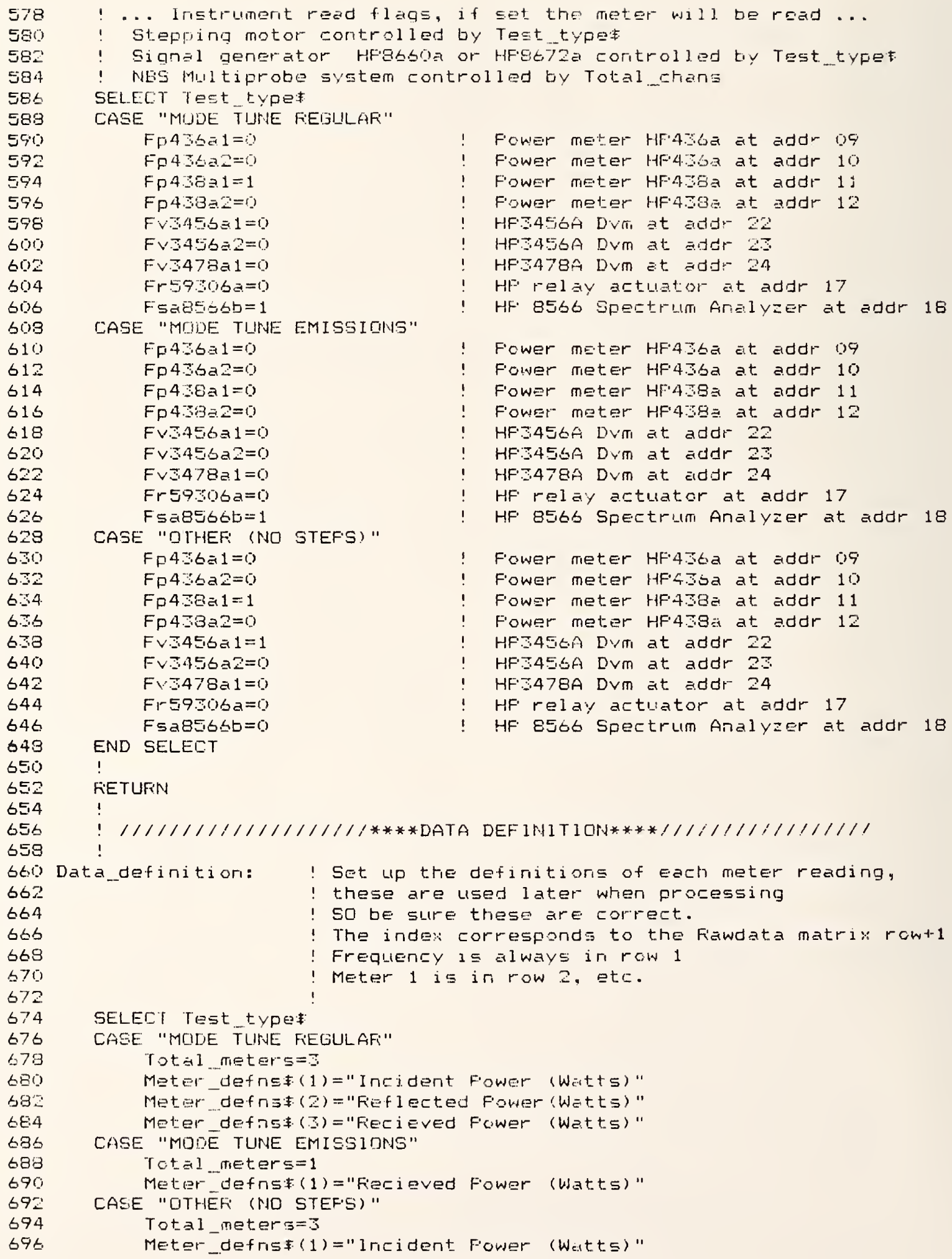


Meter defnst (2) = "Feflected Fower (Watts)"

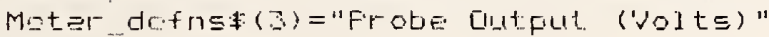




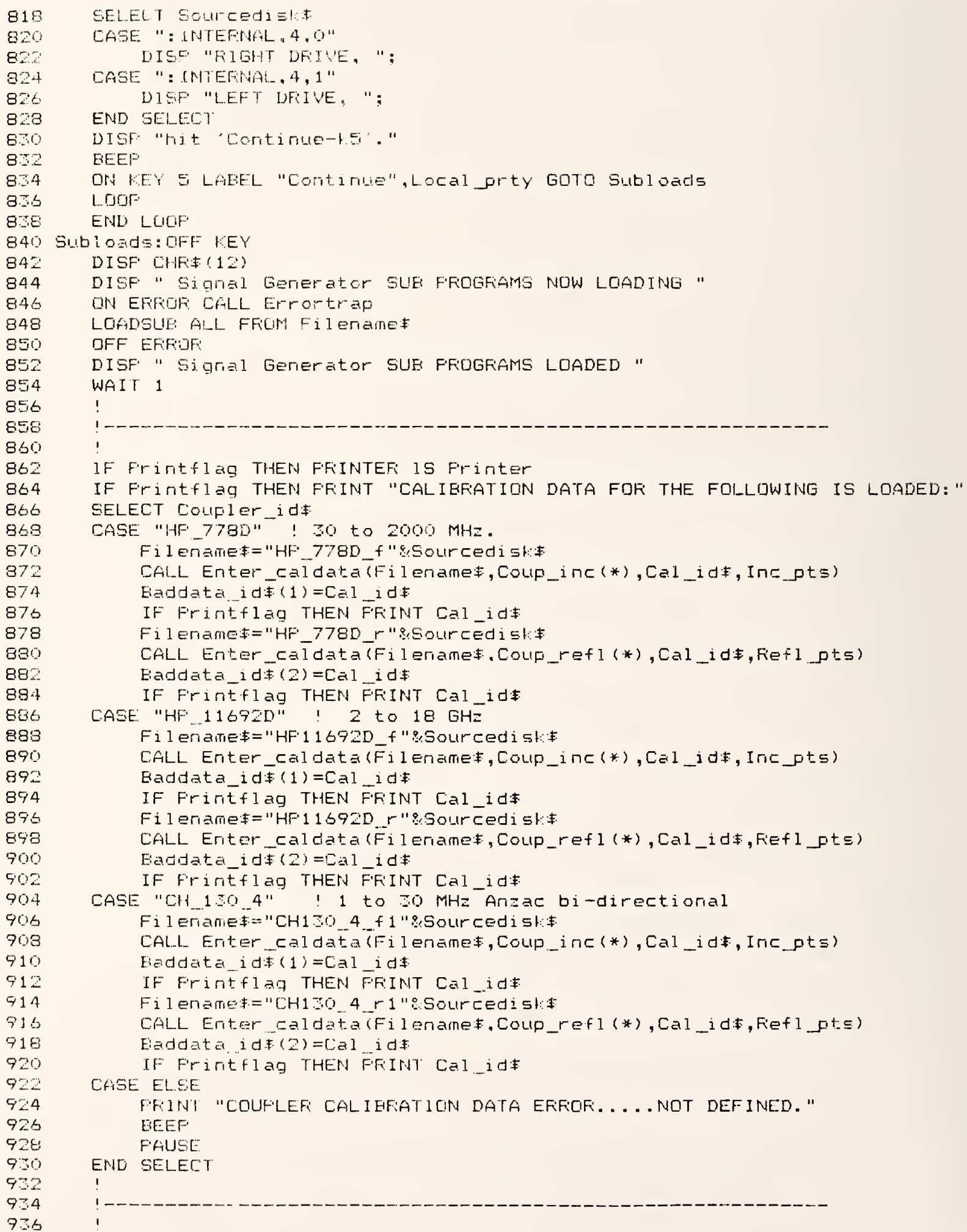




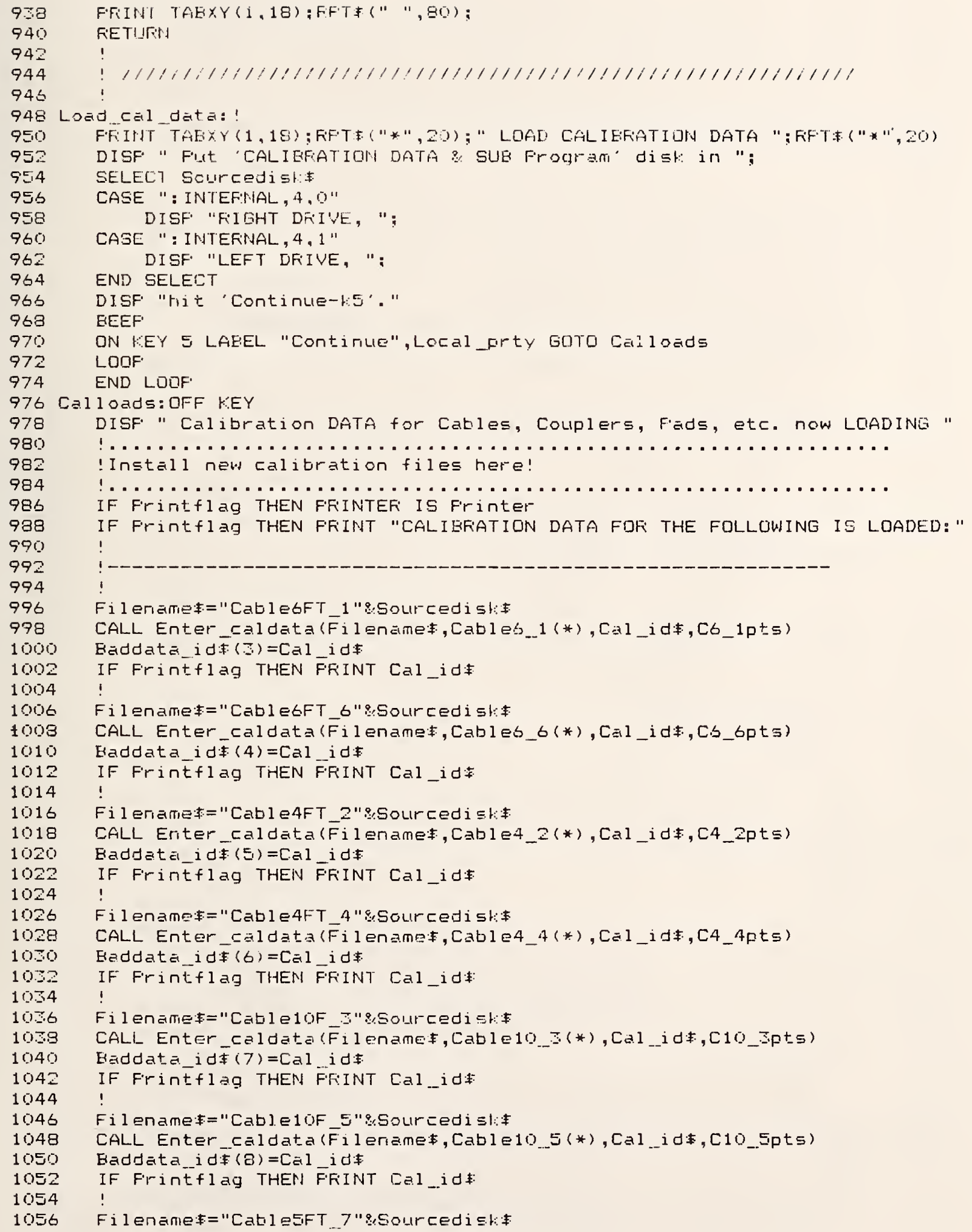




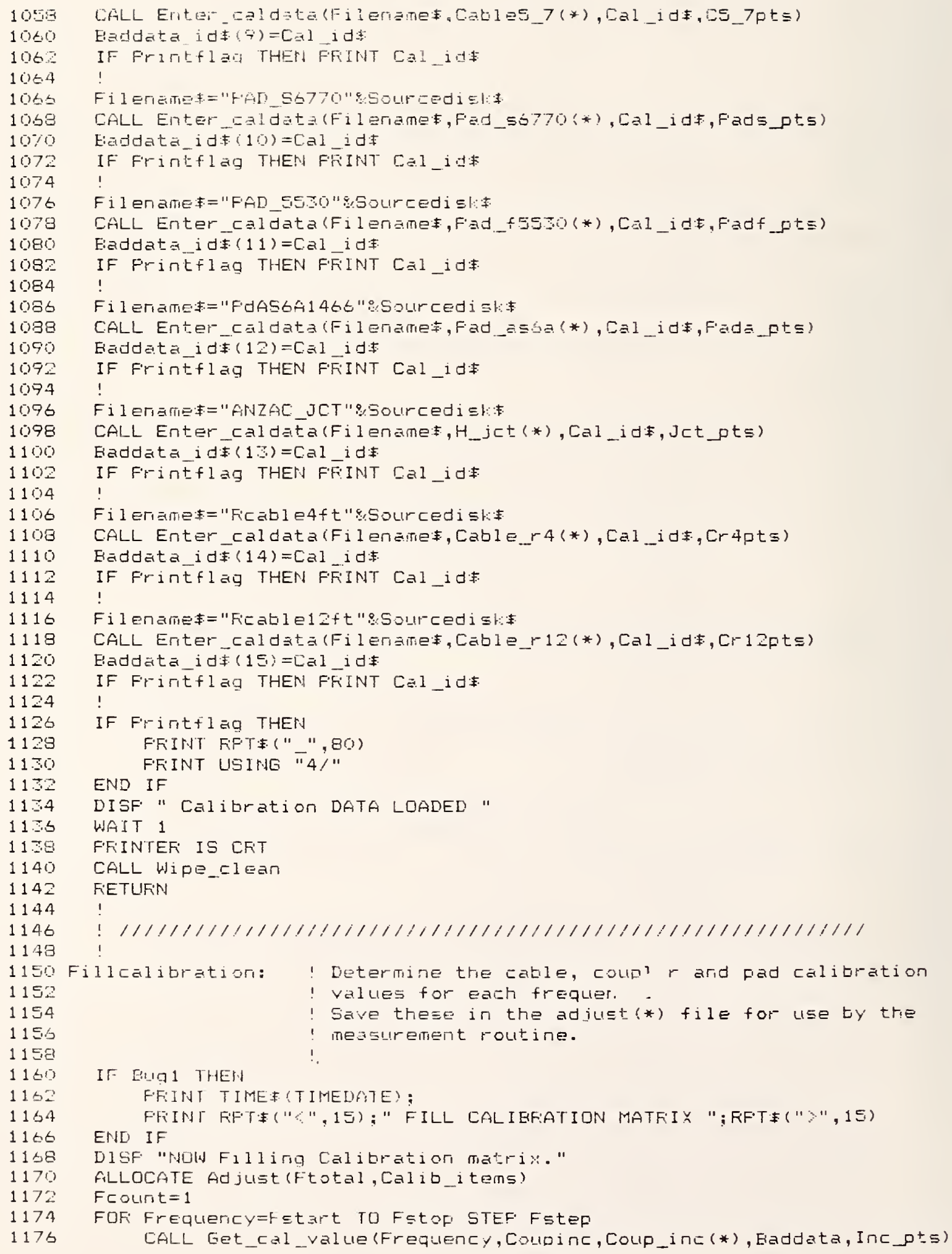


1178

118

$115=$

1184

$118 t$

1188

1190

1172

1154

119.

1198

1200

1202

1204

1206

1208

1210

1212

1214

1216

1218

1220

1222

1224

1226

1228

1250

1252

1234

1236

1238

1240

1242

1244

1246

1248

1250

1252

1254

1256

1258

1260

1262

1264

1266

1268

1270

1272

1274

1276

1278

1280

1282

1234

1236

1288

1290

1292

1274

1296

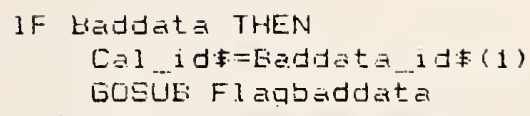

Ad_iust (FCount, 1 ) = Coufi inic

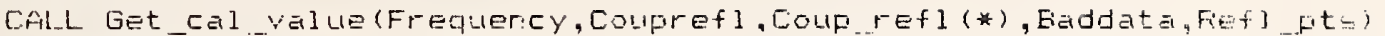

IF Baddata THEN

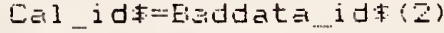

END $1 F$ GOSUH Flagbaddata

Ad just (Fcount,, 2 ) = Coupref 1

!

[if́lLL Get_ca]_value (Frequenicy,C6_110s5, Cableb_1(*), Eiaddata,Cb_1pts)

IF Baddata THEN

Cal idt=Laddata id (

GUSUE Flagbaddata

END IF

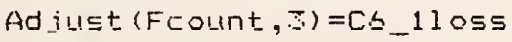

CALL Get cal value (Frequeney, C6_bloss, Cableb_6(*), Baddata, Co_bfts)

IF Baddata THEN

Cal idf=Eaddata_idt(4)

END IF GOSÜB Flagbaddāta

Adjust $($ F count, 4$)=$ C6_S1055

!

CALL Get_cal_value (Frequency,C4_2los5,Cable4_2(*),Eaddata,C4_2pts)

IF Eaddata THEN

Cal_id:F=Eddata_idF(

GDSUE Flabbaddata

END IF

Adjust (Fcount, 5 ) $=[4$ __2los

!

CALL_Get_cal_value (Frequency,C4_410ss,Catie4_4(*), Eaddata, C4_4Fts)

IF Baddata THEN

Cal idt:=Baddata idt(b)

Gusü Flagtaddata

END IF

Ad just (Fcount, 6$)=C 4 \_410 \leq 5$

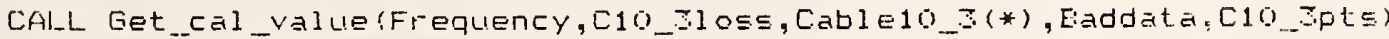

IF Baddata THEN

Cal id =Eaddata id (7)

EYHD IF

GOSIJUE Flagtaddata

Adjust (Fcourit, 7 ) = D 10 _

!

CAl-L Get_cal_value (Frequency,C10_5loss,Cable10_5(*), Baddata,C10_5pts)

IF Eaddat? THEN

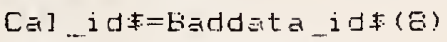

GOSUE! Flagtaddata

END IF

Ad just (FEount , 8) =C10_510SS

CALL Get_cal Value (Frequency,C5_71055,Cables._7(*), Eladdata,C5_ypts)

IF Baddata THEN

$C a 1$ idt=Faddata_idf(9)

END IF 
Ad just (Fcount, 9) $=$ C5 71053

!

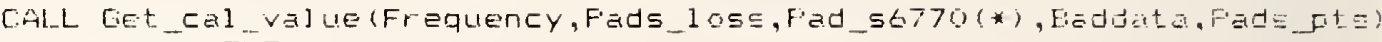
IF Baddata THEN 


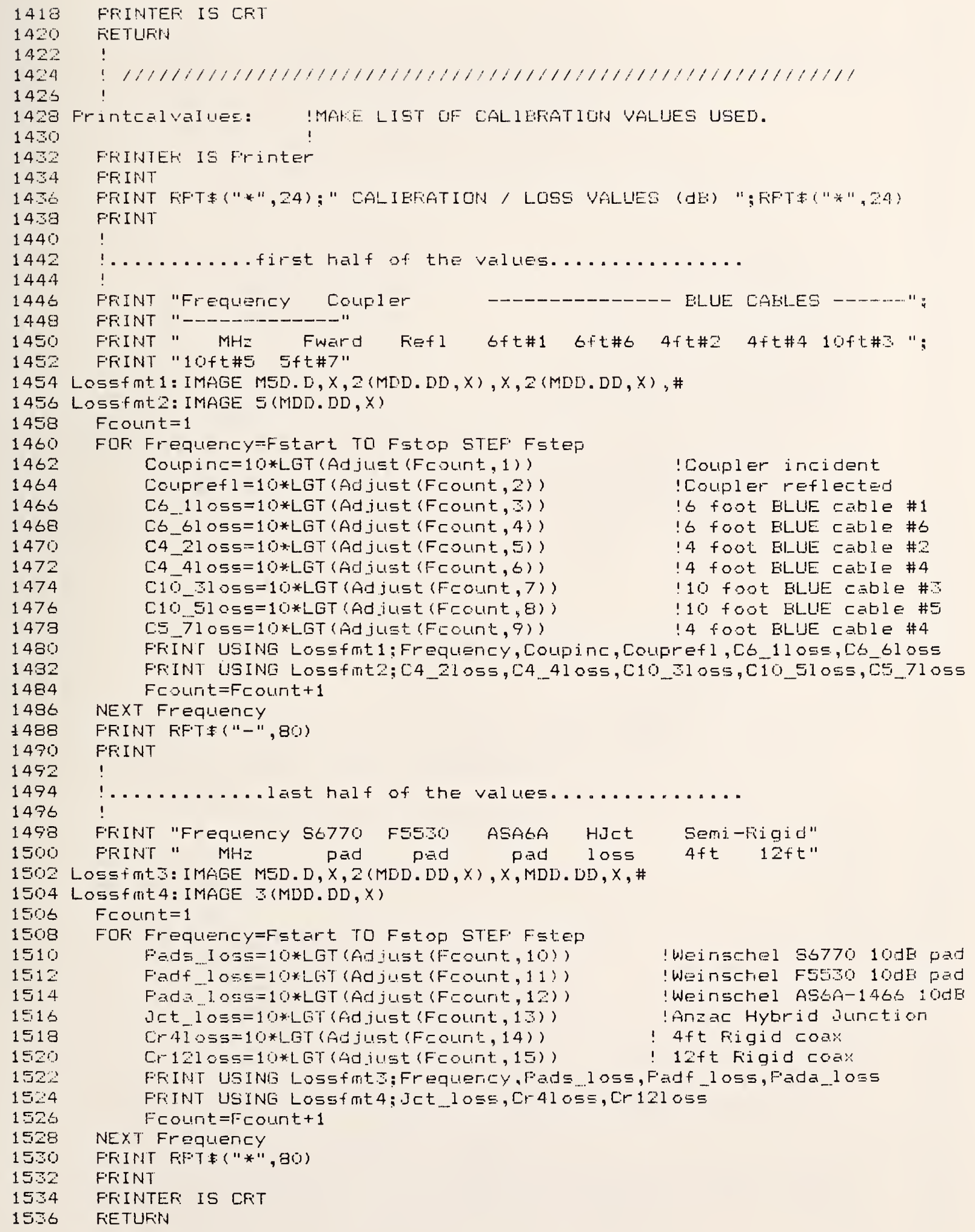




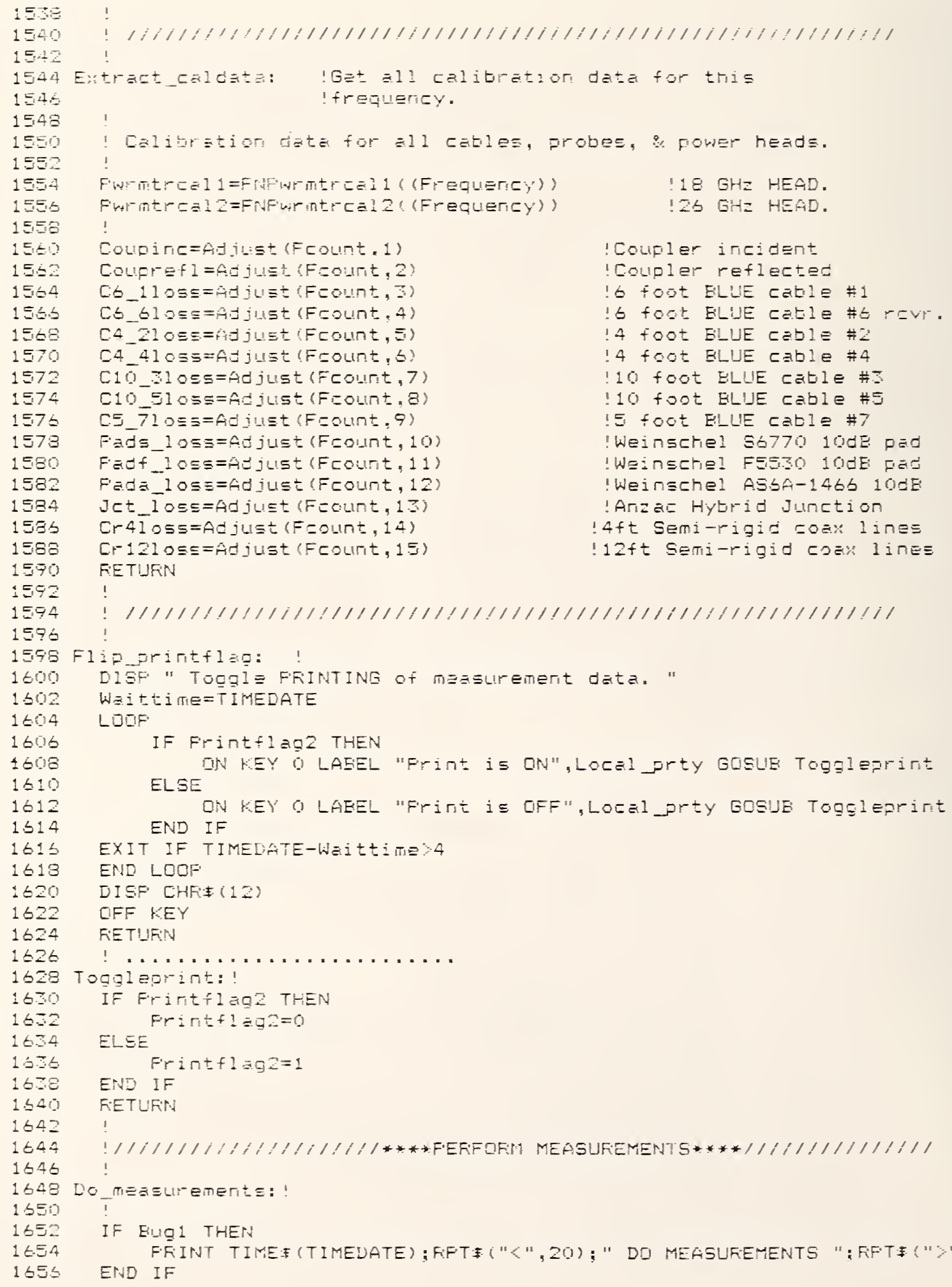

Calibration dzta for ell catles, probes, ․ fower heads.

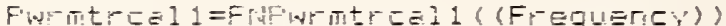

Futhtrealz=Fifiwrintrca12( (Frequericy))

Coupiric=Ad, ils: (Fcount, 1)

Couprefl=AdjuEt (F:Ount, 2 ?

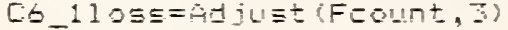

Co_blosミ=Adjust (Foount, 4 )

C. $4210 \equiv \equiv=$ fidjust (Fcount,

C4 410 ss=Adjust (Foount, s)

C10_-3oss=Adjust (FCOLI, 7 )

C10 $51055=$ Adjust (Foulint, B)

C. 7 ?

Fads_loss=Adjust (Foount, 10 )

Fiadf loss=Adjust (Fcourt, 11 )

Fiads_los $=$ Ad just (Fcount, 12 )

Jct_Los=Adjust (FEount, 1 I

Cr 4 Ioss=Adjust (Foount, 14)

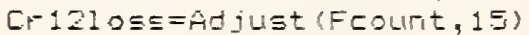
FETUFN

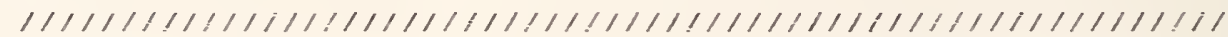

Flif printflag: !

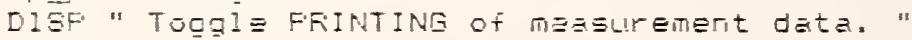
Waittine=TIMEDATE LOQF.

If Frintflag2 THEN

ELSE

IN KEY O LAEEL "Frint is ON", Locel grty GUSUB Togglgarint END IF

ON KEY O LAEEL "Fririt iE GFF" "LOEEl prty GOSUE TOggleprint

EXIT IF TIMELATE-Waittime>4

END LOOF

DISF CHF:P(12)

DFF KEY

FEETUFIN

Toggleprint:

IF Frititflagz THEN

ELSE

Frintflig $=0$

END IF

Frintflag $2=1$

FEETUFN.

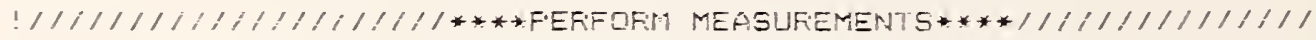

Lo mミミミur-ementミ:

IF EUII THEN

END IF 
1658

1660

$166=$

16.54

1666

1668

1670

1672

1674

1676

1678

1680

1682

1684

1686

1.583

1690

1692

1694

1696

1698

1700

1702

1704

1706

1708

1710

1712

1714

1716

1718

1720

1722

1724

1726

1728

1750

1732

1734

1736

1738

1740

1742

1744

1746

1748

1750

1752

1754

1756

1758

1760

1762

1764

1766

1768

1770

1772

1774

1776

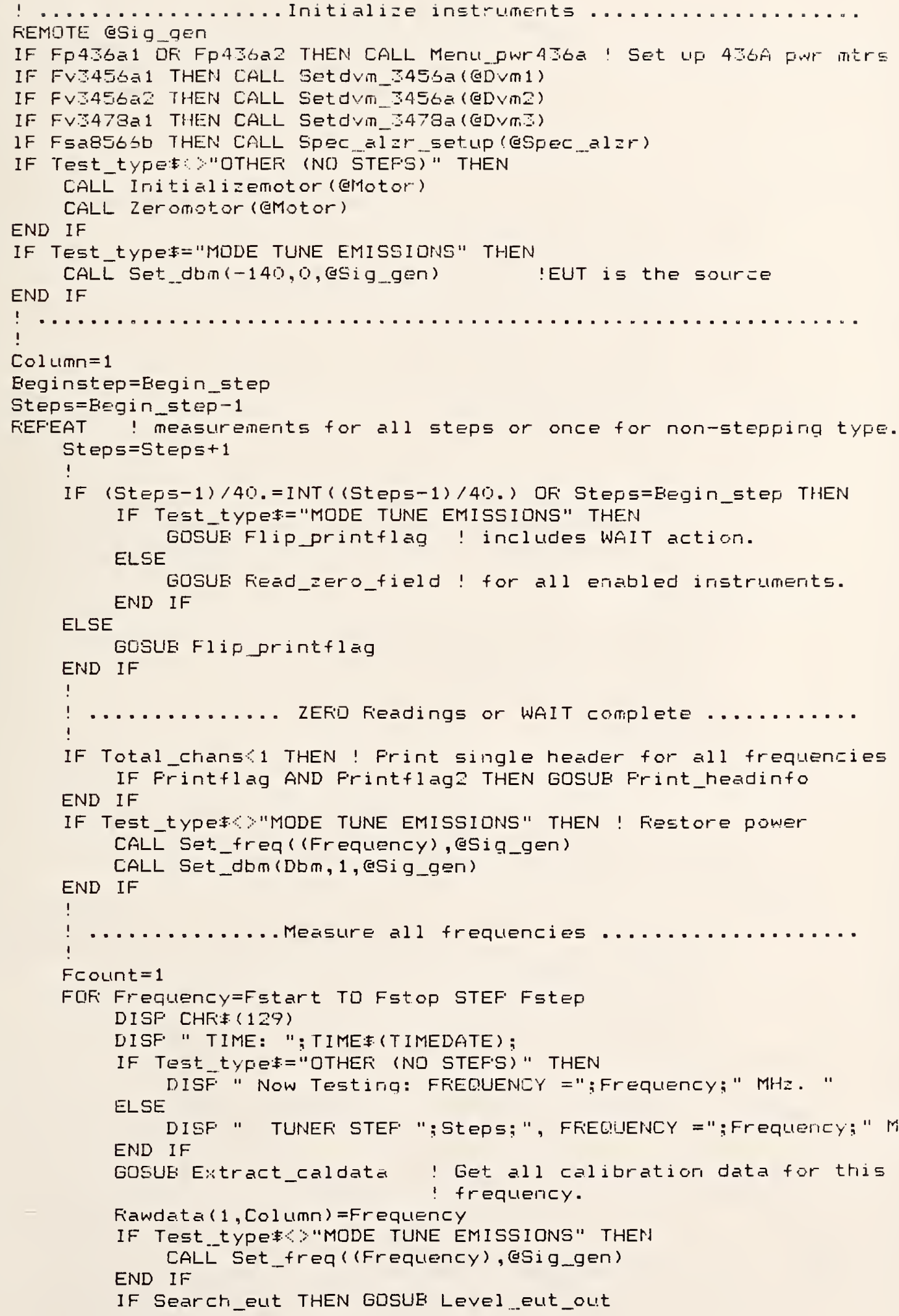




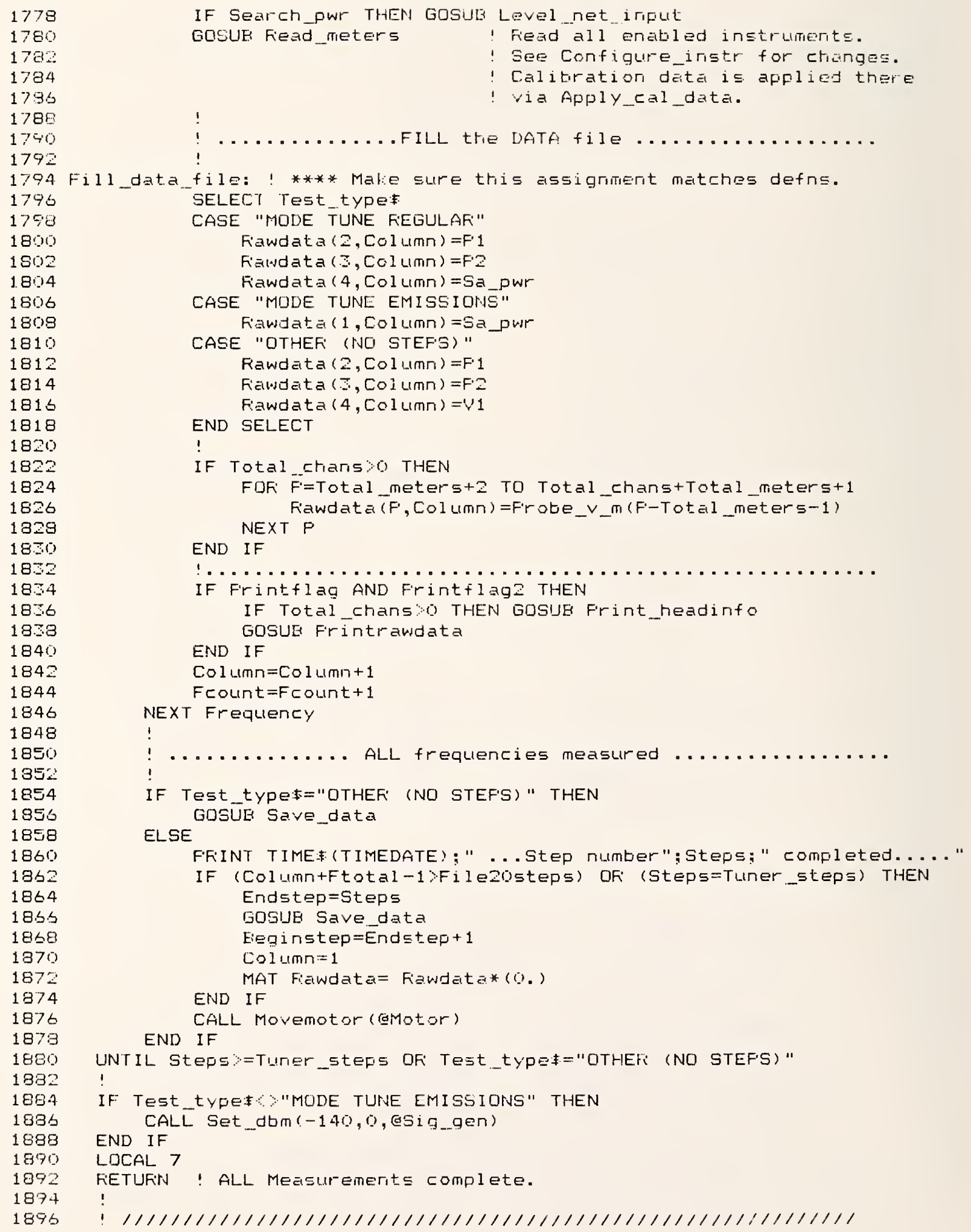




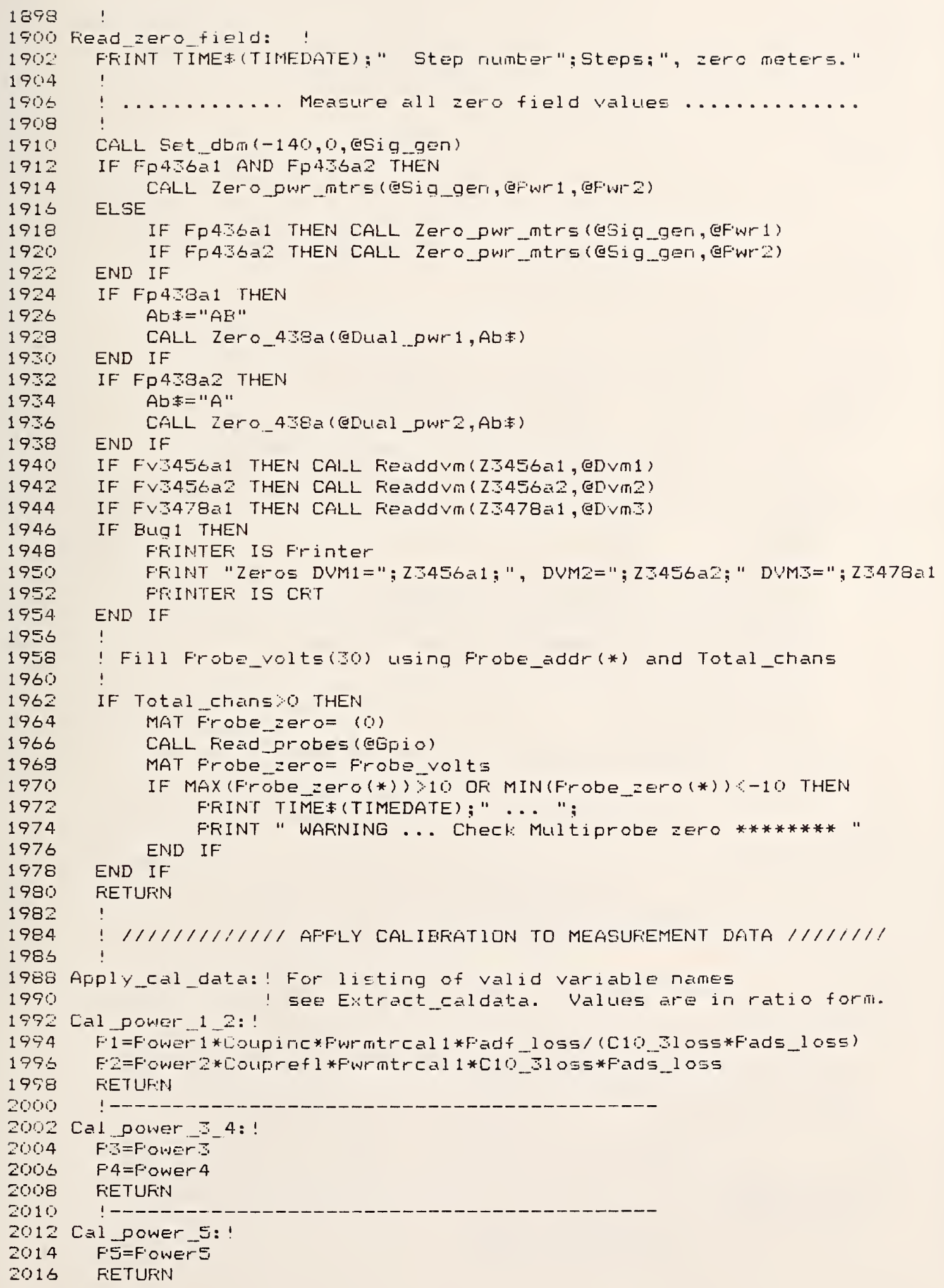




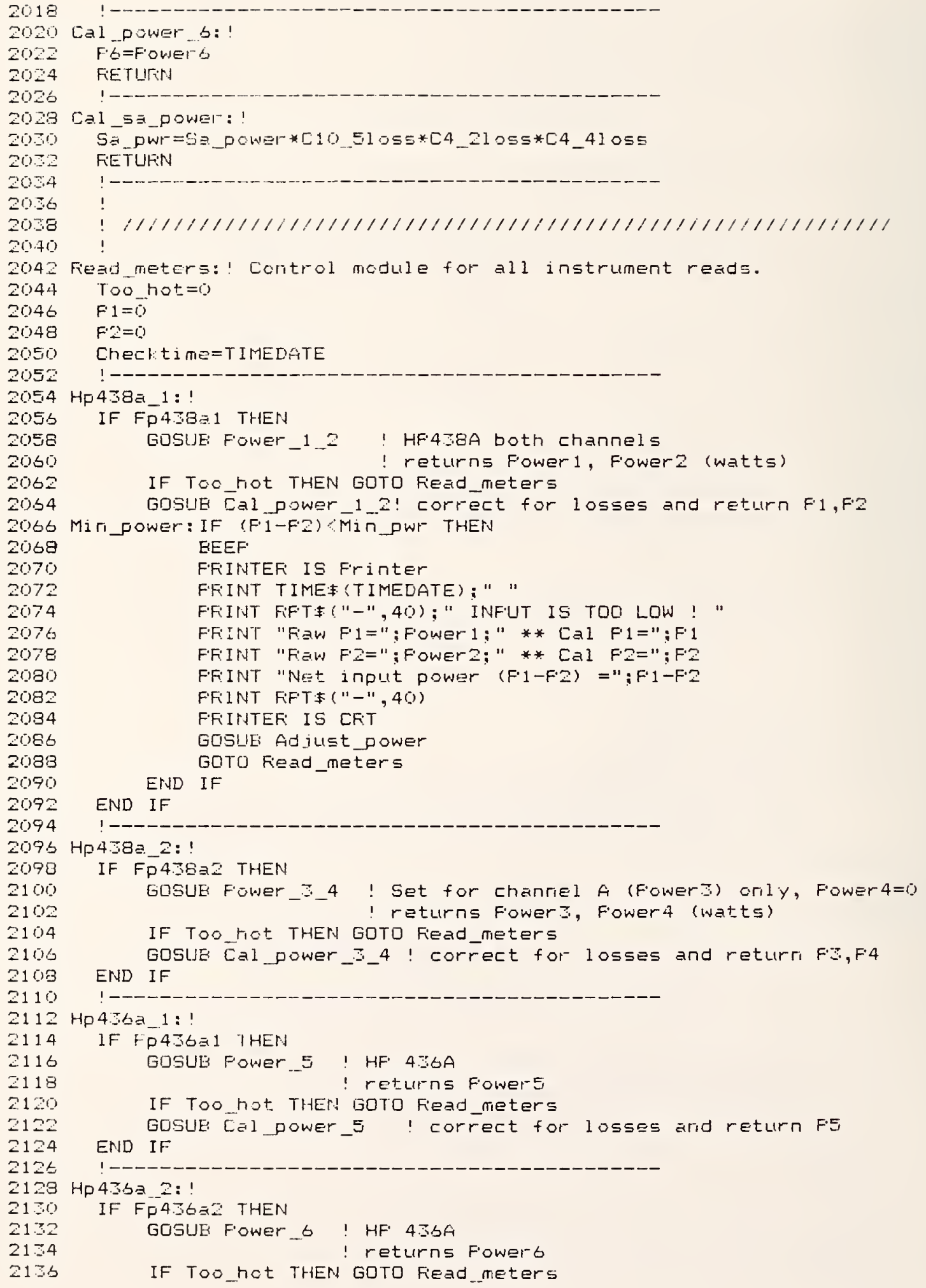

$$
\text { END IF }
$$




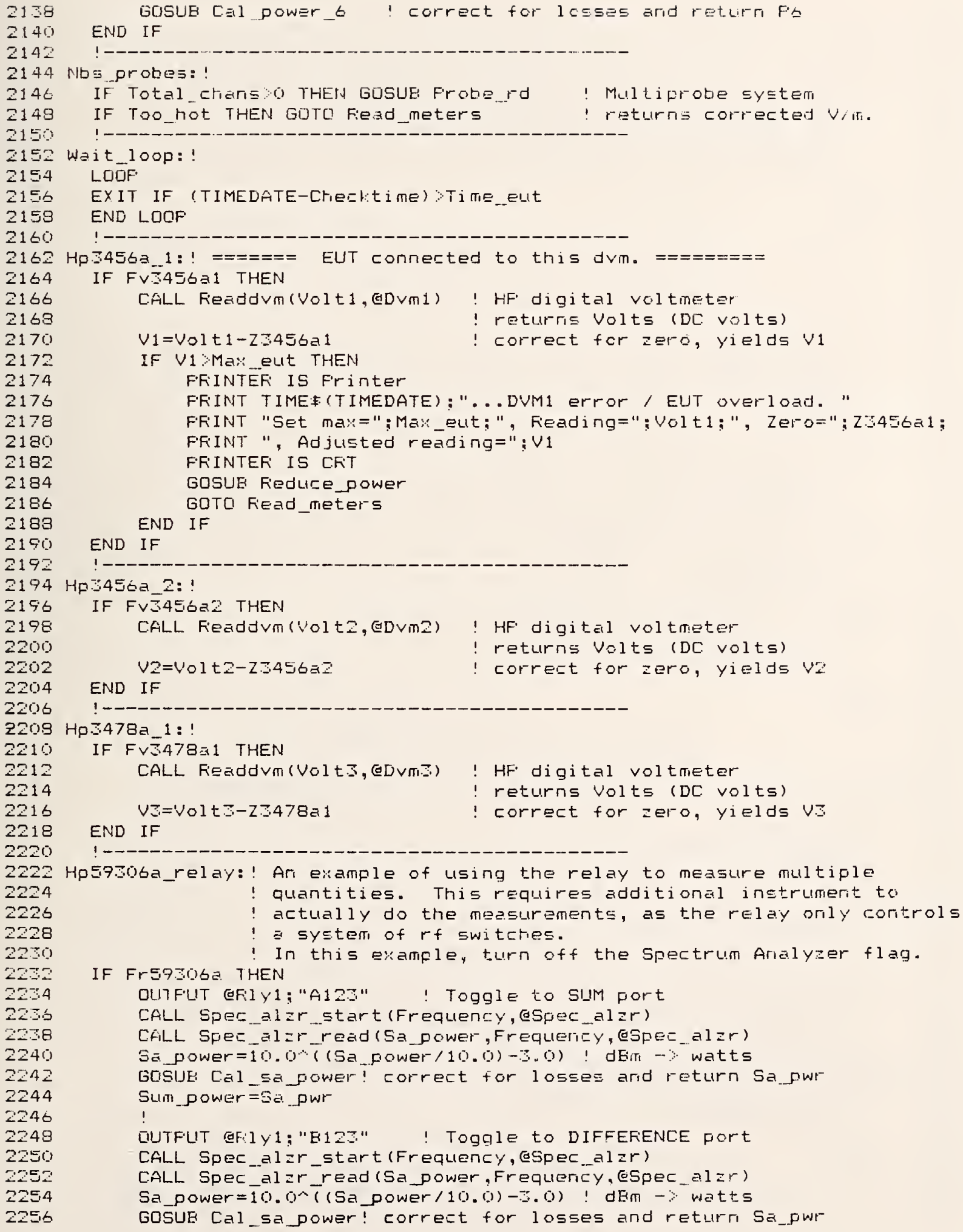




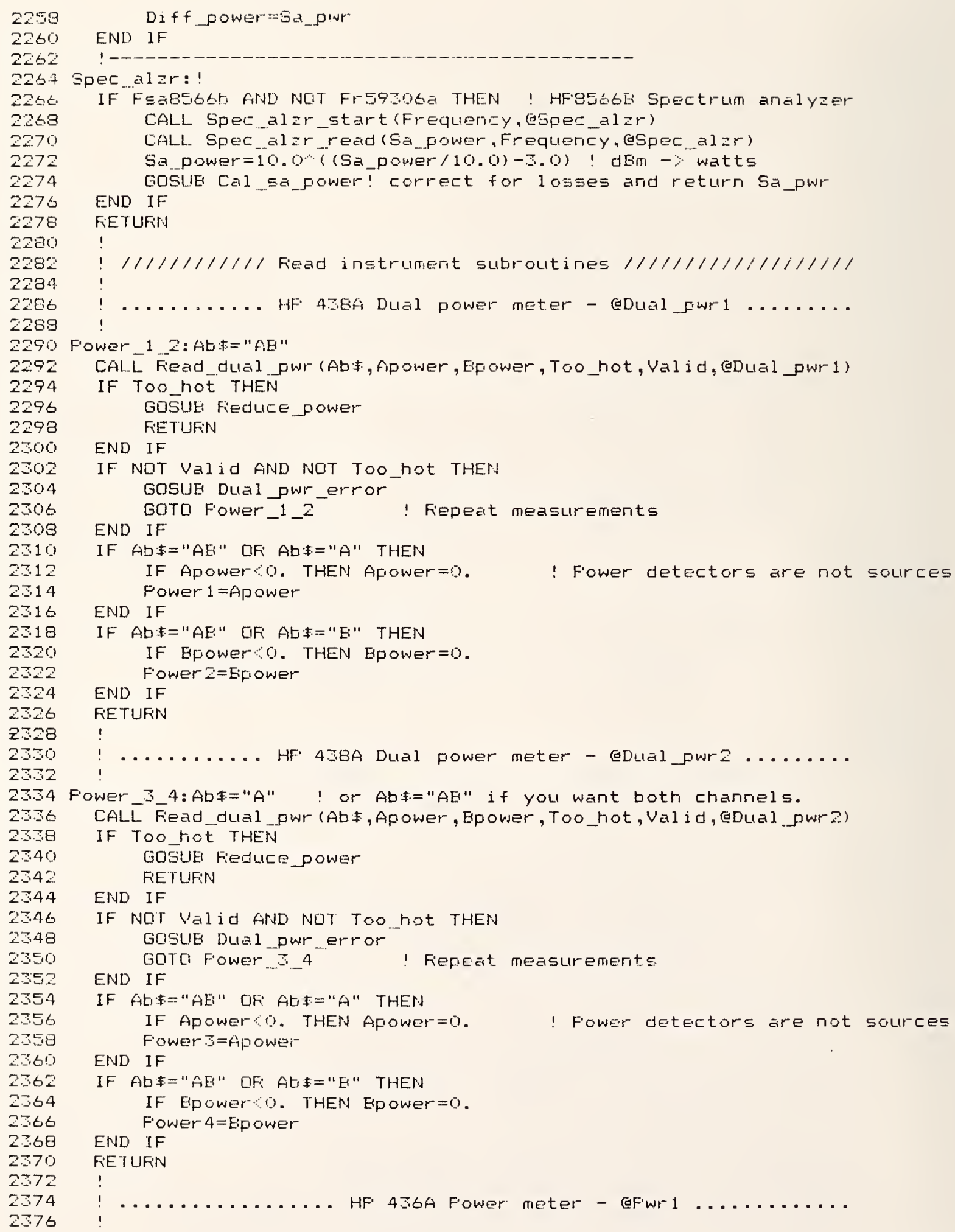

END $I F$

Diff power=sa_pinr

: pec al ar:

IF FEa85bst fND NGT Fr.5930sa THEN ! HFBSEGE SFEctrum analyaer

CALL Spec_alar_start (Frequency. (aspec_alar)

CALL Spec alar read (Sa fower, Frequency, eSpec alar)

Sa_power=10.0\%(Sa_power/10.0)-3.0) ! dEm - $)$ watts

END IF

GUSUE Cal sa_fower! correct for losses and return sa_pwr

FEETUFIN

$!$

////////// Fiead instrument subroutines /////////////////

!.......... HF 4.3Ea Dual power meter - eDual frwr $1 \ldots . . .$.

Fower_1_2: At:

CALL Fiead_dual__pwr (AD:, fipower, EFower, Too_hot, Valid, GDual_pwr 1)

IF ToO hot THEN

GOSUE FiedLICE fower FETUFN

END IF

IF NOT Valid AND NOT Too_hot THEN GOSUE Dual pwr_error

END IF GOTO Fower_ $12^{2}$ ! Fiepeat measurements

IF Ab:="AE" $\square F$ Ab:="A" THEN IF Apower 0. THEN Afower=0. ! Fower detectors are not sources END IF Fower 1 = Ap ower

IF Ab:="AE" GFi AET:="E" THEN

IF EPower 0 . THEN EPower $=0$.

END IF Fower $2=$ Efrower

FiETUFIN

!

!....... HF 4SBA Dual fower meter - GDilal purz ......

Fower_3_4:Abt:="A" ! or $A b:=$ "AB" if you want both channels.

CALL Fiead_dual_fowr (Ab $\$$, Apower, Epower, Too_hot, Val id, (eDual.pwr2)

IF TOO rot THEN

GOSUE FiedLICE power

END IF

FIETUFIN

IF NGT Valid AND NOT Too hot THEN GOSLIE Dulal_pwr error

END IF GOTO Fower.._. 4 ! Fiefeat measurements

IF ALE:="AE:" OF: AUt:="A" THEN

IF Apower o. THEN Apower-0. ! Fower detectors are not sources

END IF

IF $A B:=$ "AE" $\square F: A E:=" B "$ THEN

IF Épower O. THEN Epower $=0$.

END IF

Fower $4=$ Epower

FETUFIN

! 
2378 Fiower $5:$ ! Fiw idt: "F'1"

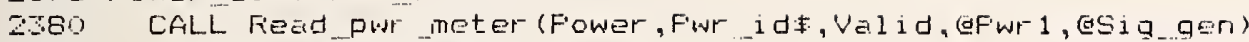

2382 IF NOT Valid THEN

2405 Fower_6: ! Fwr_id:= "F'6" 


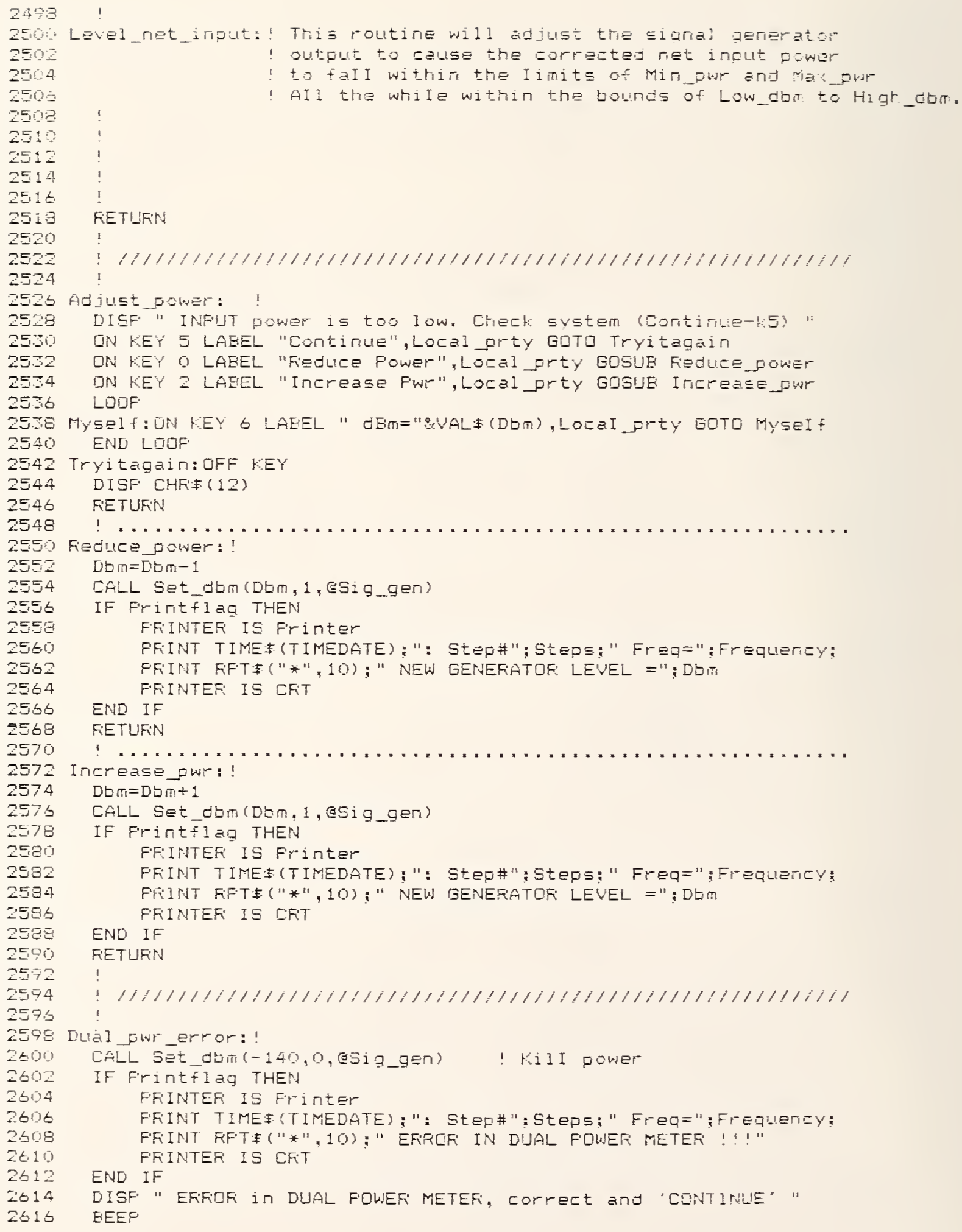

F 


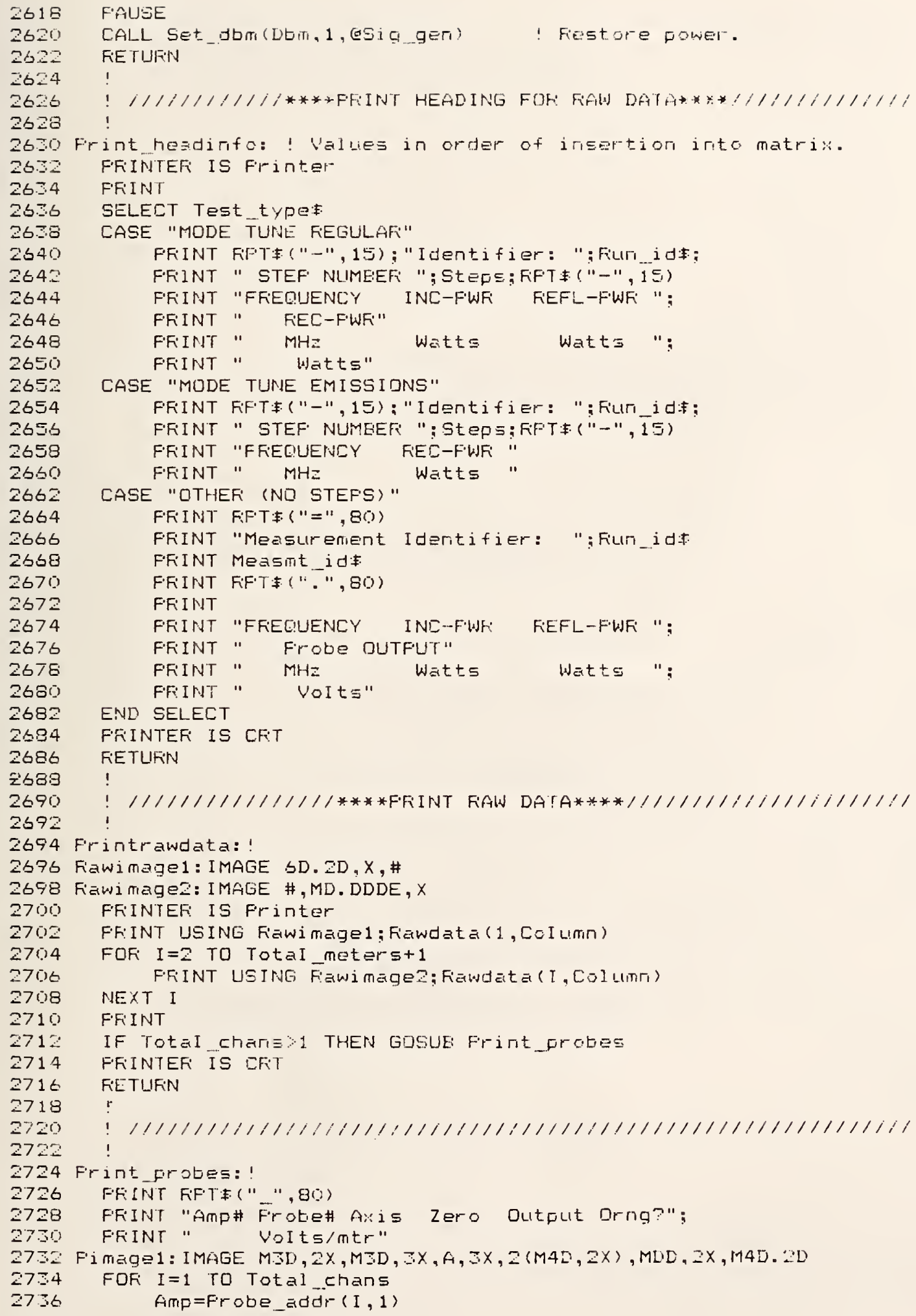




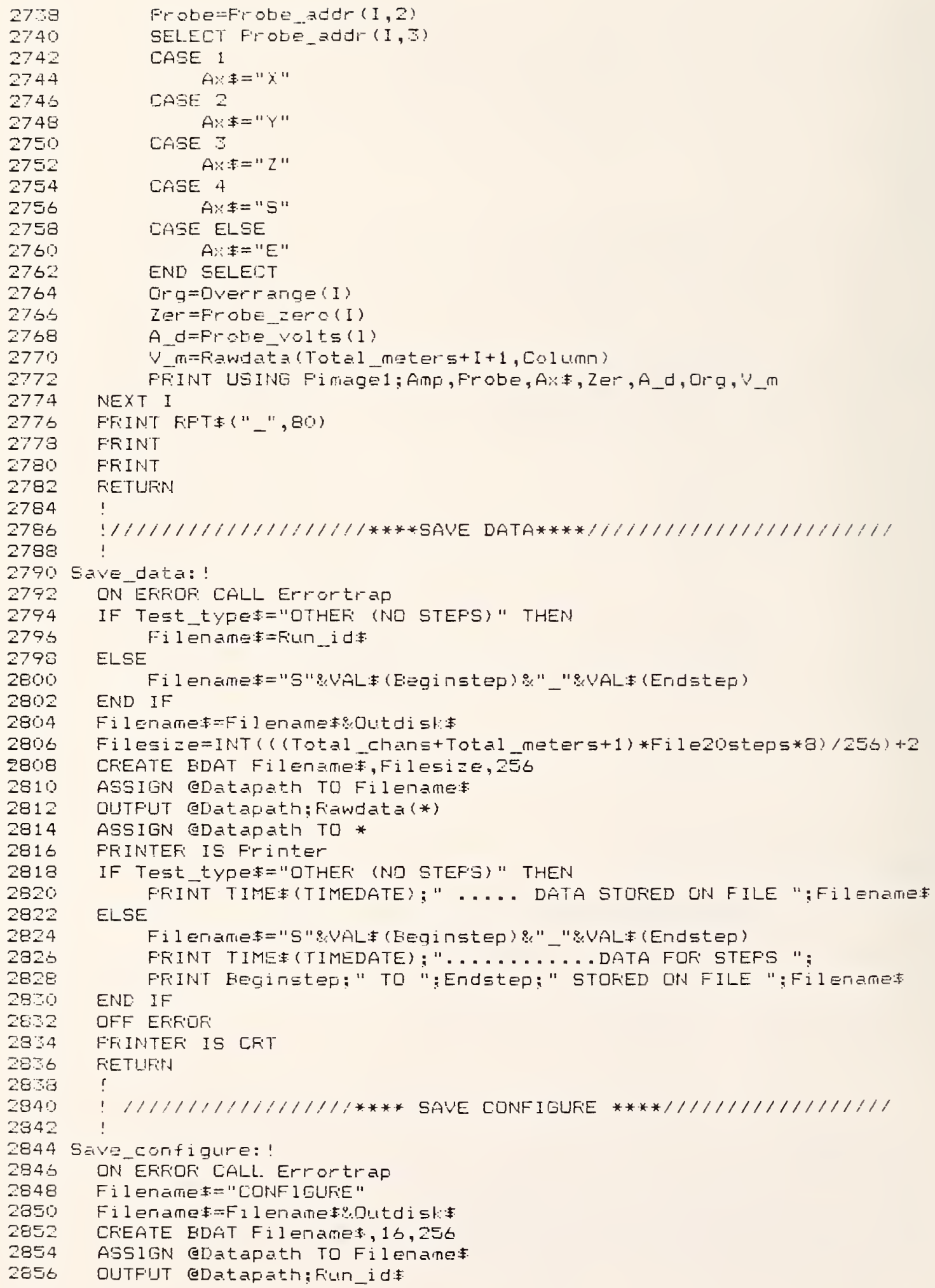


2855

2850

$28=2$

$286-7$

28 .

2868

287

2872

$2 \Xi 74$

2876

2878

2880

2852

2834

2886

2883

2890

2372

2694.

2896

2393

2900

2902

2907

2906

2908

2910

2712

2914

2916

2918

2920

2522

2924

2726

5928

2930

2932

2954

$29: 6$

2958

2940

2942

2744

2945

2949

2050

2752

2954

2756

2958

2760

$29 \leqslant 2$

2764

$29 t=$

2968

2970

2772

2974

2976
OUTFUT GDatapath; Measmt id id

CIJTFur GDatepatr; Time datet.

GUTFuT [01 atapath; Fistart, Fstop, Fstep

IF Test tYpet="DTHEF: (TO STEFS: "THEN

QUTFIT (aDatapath: 1

ELSE

DUTFUT GDatapatri; Total_Eteps

END IF

DUTFUT ODEtagath; Total meters

DUTFIT EDatapath; Tota] chans

OUTFUT GDatapath; Frobe addr (*)

UUTFUT GDatapath:Meter _defrist(*)

OUTFUT @D

Assigrl eD Etapath TC *

OFF EF:FiOF:

FFIINTEF: IS CFT

FFIINT TIME: (TIMEDATE):" CONFIGUFE file for "; Fil!n_idf;" SAVED." FEETUFit

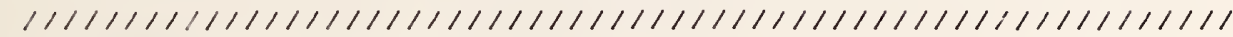

Fint vitals: !

FFINTEF: IS Firinter

FFINT FiFT:(" ", 80)

FFINT "THIS DATA SET IS IDENTIFIED AS: "; FiLIn_id

FFINT "MEASUREMENT date: "; Time_dateq.

FFIINT FiF'T $("-", 80)$

FFIINT Measint_id:

F.FIINT F.F.T. "-" , 80$)$

FRINT "FFEQUENCIES "; Fstart;" TD ";

FFIINT FStop; "STEF ";Fstep;" MHE."

FFIINT "THE TUNEF: STEFF'ED "; Total steps;" increments."

FFildT Total meters;" FWF: METEFS and DVMs, along with "; Total_chans;

FFIINT " charinels of the NES multiprobe system."

FFIINT

FFIINT "ThE meEsured DATA are defined as follows:"

FRINT FETT:("-", B(0)

FFINT "Data slot \# Description";

FFINT TAE (40); "Data slot \# Description"

$J=I N T$ ! ( (Total chans+Total meters $) / 2)+1)$

IF $\mathrm{J} \gamma(($ Total_chans+Total_meters $) / 2)+1$ THEN $\mathrm{J}=\mathrm{J}+1$

$I=1$

$\hat{r}=\mathrm{J}$

FEEFEAT

! J is starting point of second columr.

FFIINT I; ": "; Meter defnst.(I);

IF $r$ Total chanst Total_meters THEM

ELSE

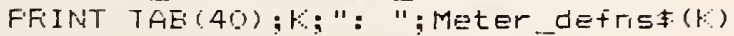

FFFINT

END IF

$I=I+1$

$\therefore=1+1$

UNTIL I\%=J

FFi INT FFTT ("-", OO)

FFIINT USINE "こ/"

FFIINTER IS CRT

FEETLIFIN

11111111111111111111111111111111111111111111111111111111 


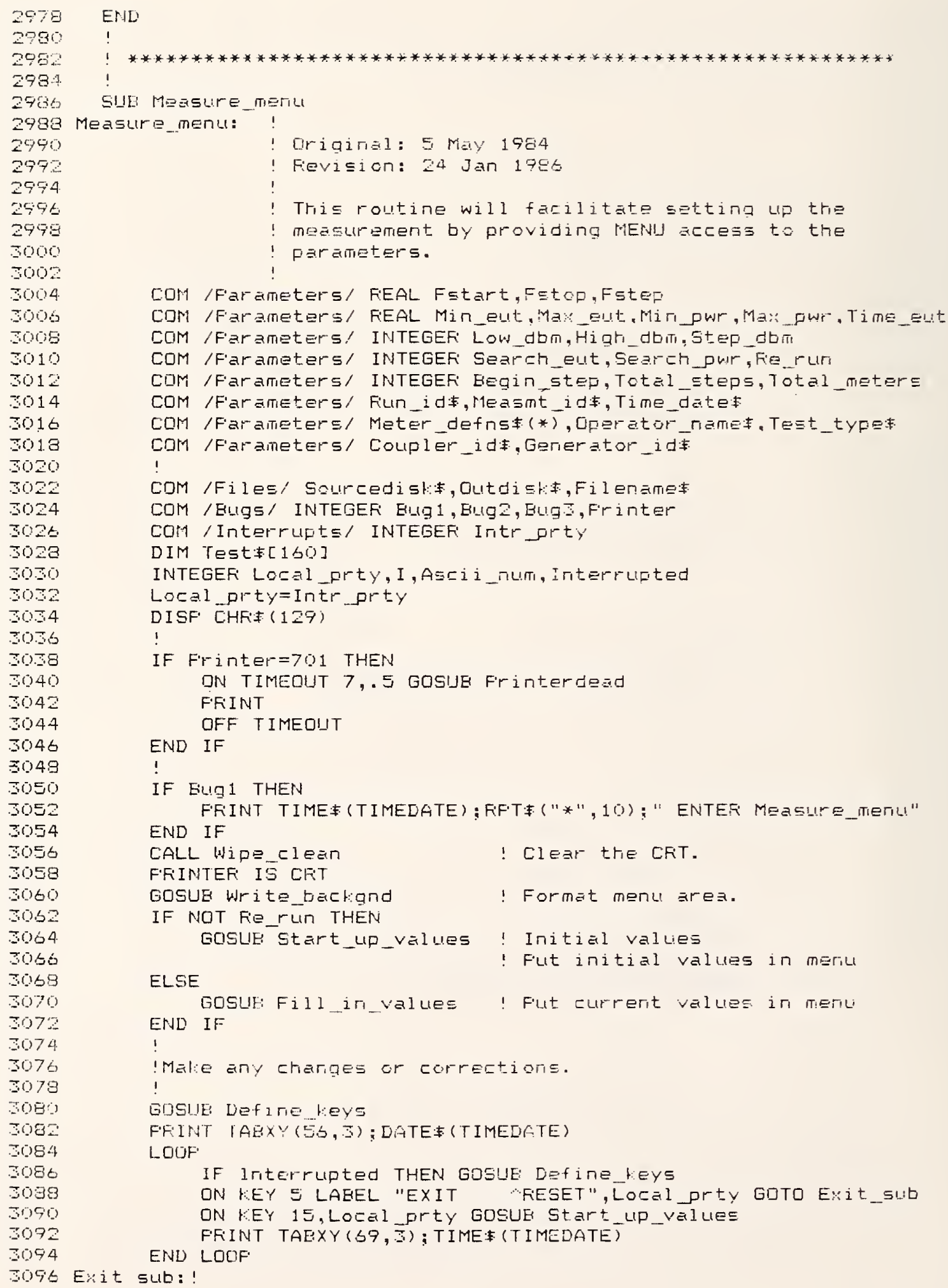









\begin{tabular}{|c|c|}
\hline 3058 & OFF $\mathrm{EEY}$ \\
\hline 2100 & DISF CHFt:(12) \\
\hline 5102 & Time date $=1$ IME (TIMEDATE) 点", "E[ATE: (TIMEDÍTE) \\
\hline$\$ 104$ & FFIHTER IS Frinter \\
\hline $310 t$ & IF Frinte:-701 THEN DUMF FILFHA \\
\hline 103 & IF ENUG 1 THEN \\
\hline 110 & 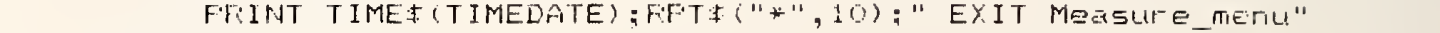 \\
\hline 112 & END IF \\
\hline$\$ 114$ & CFiLL Wifie_clean \\
\hline 113 & FFINTER IS ERT \\
\hline 118 & SUEEXIT \\
\hline 120 & $!$ \\
\hline 122 & 11111111111111111111111111111111111111111111111111111111 \\
\hline 124 & ( \\
\hline 126 & Defirie_tieys:! \\
\hline 128 & GIFF $\mathrm{KEY}$ \\
\hline 3130 & Interrupted $=0$ \\
\hline 312 & SELECT FPAFIAMETEFi TO CHANGE "; \\
\hline 134 & DISF " ("shift key) ":FFT:" " ", 15) \\
\hline $51: 6$ & ON $\because E Y$ O LAEEL "TEST ID NYFE", Local prty GOSUE ChangE_eltid \\
\hline$\div 1.8$ & ON FEY 10, Local prty GOSUE Change type \\
\hline 140 & ON IEY 1 LAEEL "FFEDS "SIG GEN", Local prty GUSUE Change_freqs \\
\hline$\$ 142$ & ON FEY 11 , Local prty GOSUE Change_levels \\
\hline$\$ 144$ & ON FEY 2 LAEEL "INFUT MIN MAX", LECAl_prty GOSUE Change_pwr_min \\
\hline 146 & ON KEY 12, Local prty GOSUE Change pwr max \\
\hline$\$ 148$ & ON FEY I LAEEL "LEVEL "SEAFICH",Local_prty GOSUE Change_search_p \\
\hline 3150 & ON FEY 1S, LCCal_prty GOSLE Change_search_e \\
\hline 3152 & ON rEY 4 LAEEL "EUT MIN MAX" "LoCal_frty GOSUE Change_eut_min \\
\hline 3154 & ON FEY 14 , Local fity GOSLE Change_eut_max \\
\hline 315,6 & ON rEY o LAEEL "DATE NAME",LOCal_prty GOSUE Call_time_date \\
\hline 158 & ON FEY 16, Local prty GOSUE Enter_neine \\
\hline 3160 & ON FEY 7 LAEEL "Response Time", Local prty GOSUE Change_response \\
\hline 162 & IF TESt_tYpeFO"OTHEF: (NO STEFG)" THEN \\
\hline 3164 & ON FEY 8 LAEEL "TUNEF "EEGIN", Local prty GOSUB Change_tuner- \\
\hline 365 & ON KEY 18,Local prty GOGUE Change_beginstp \\
\hline 5168 & END IF \\
\hline 5170 & ON EEY 9 LAEEL "DISF DFIVE", Local prty GOSUE Change distidriv \\
\hline 3172 & FETUFIN \\
\hline 374 & $!$ \\
\hline 3176 & 111111111111111111111111111111111111111111111111111 \\
\hline 373 & (2) \\
\hline $\begin{array}{l}180 \\
182\end{array}$ & $\begin{array}{l}\text { Start_up_values: ! Define the initial values for all farameters. } \\
\text { Fe_runi=1 }\end{array}$ \\
\hline 5184 & ! Frequency range in MHz. \\
\hline 3186 & $F s t o p=100$ \\
\hline 188 & Fstep $=50$ \\
\hline-190 & : Signal generator level in dEm. \\
\hline 3192 & High $d b m_{1}=-10$ \\
\hline 5194 & Stef, dtin $=1$ \\
\hline 5196 & $! \quad 0=$ DO NOT auto search fot EUT response \\
\hline 198 & $! \quad 1=D O$ \\
\hline 300 & ! $\overline{0}$ DO NOT auto level the Net Input power. \\
\hline 302 & $1 \quad 1=00$ \\
\hline 3204 & ! Mirimumi output of EUT in volts. \\
\hline 306 & ! Maximum oltffut of EUT in volts. \\
\hline 308 & ! Minimum net imput power in watts. \\
\hline 310 & ! Makimum net infut power in watts. \\
\hline 212 & ! Time for EUT to respond after field is set. \\
\hline 3214 & Eegin_step $=1$ \\
\hline 3216 & !fin integer division of 3200 \\
\hline
\end{tabular}




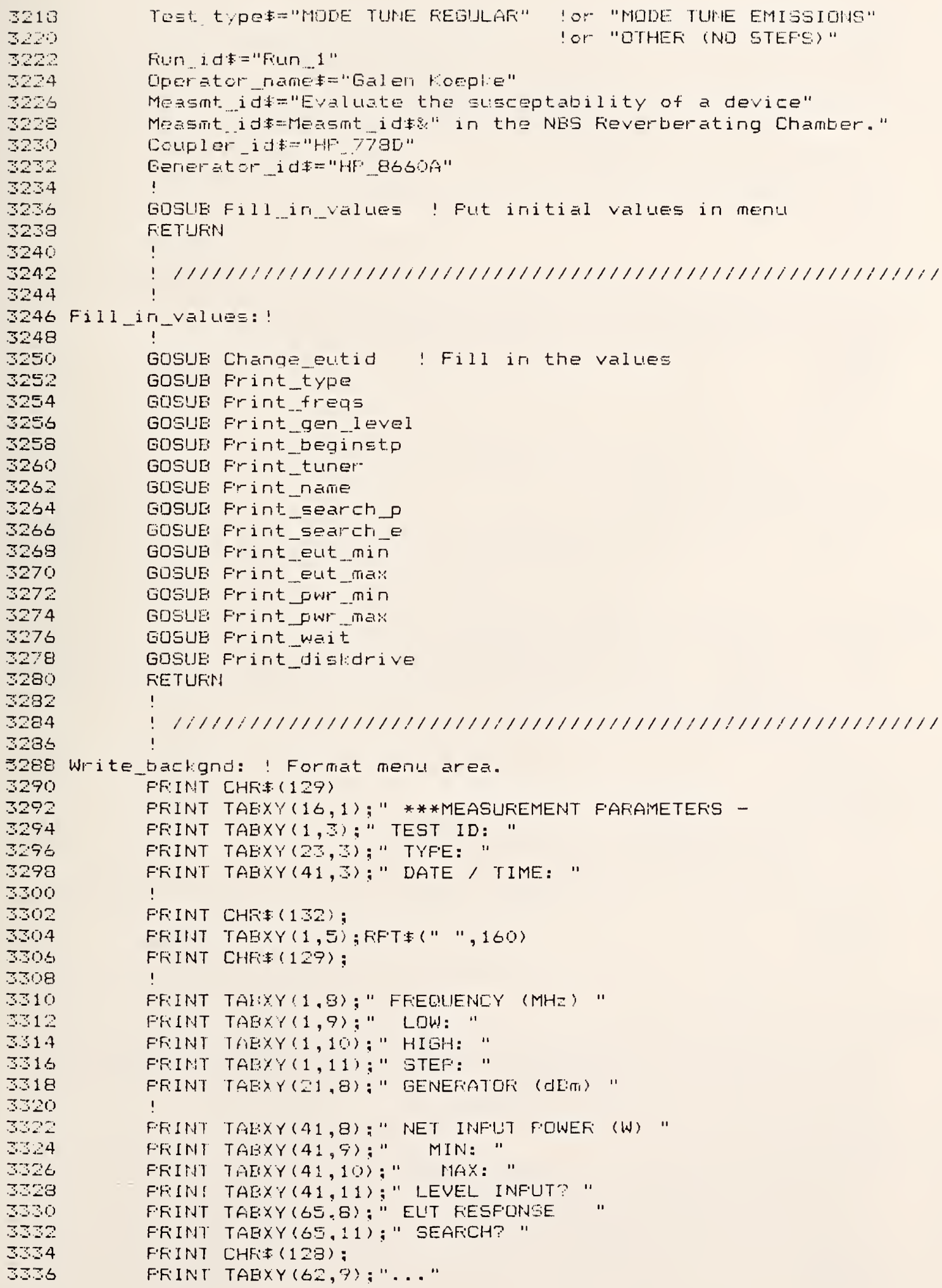




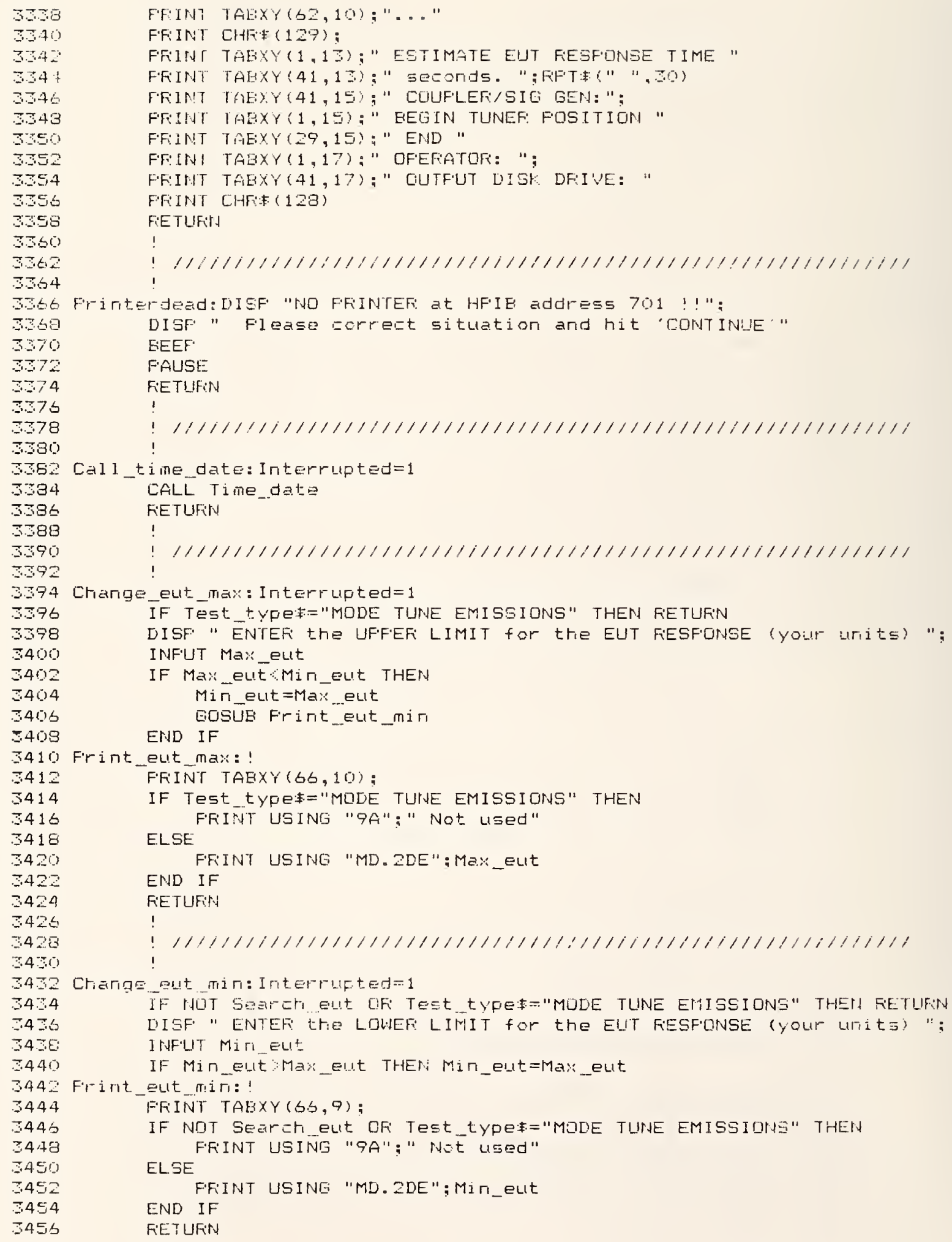




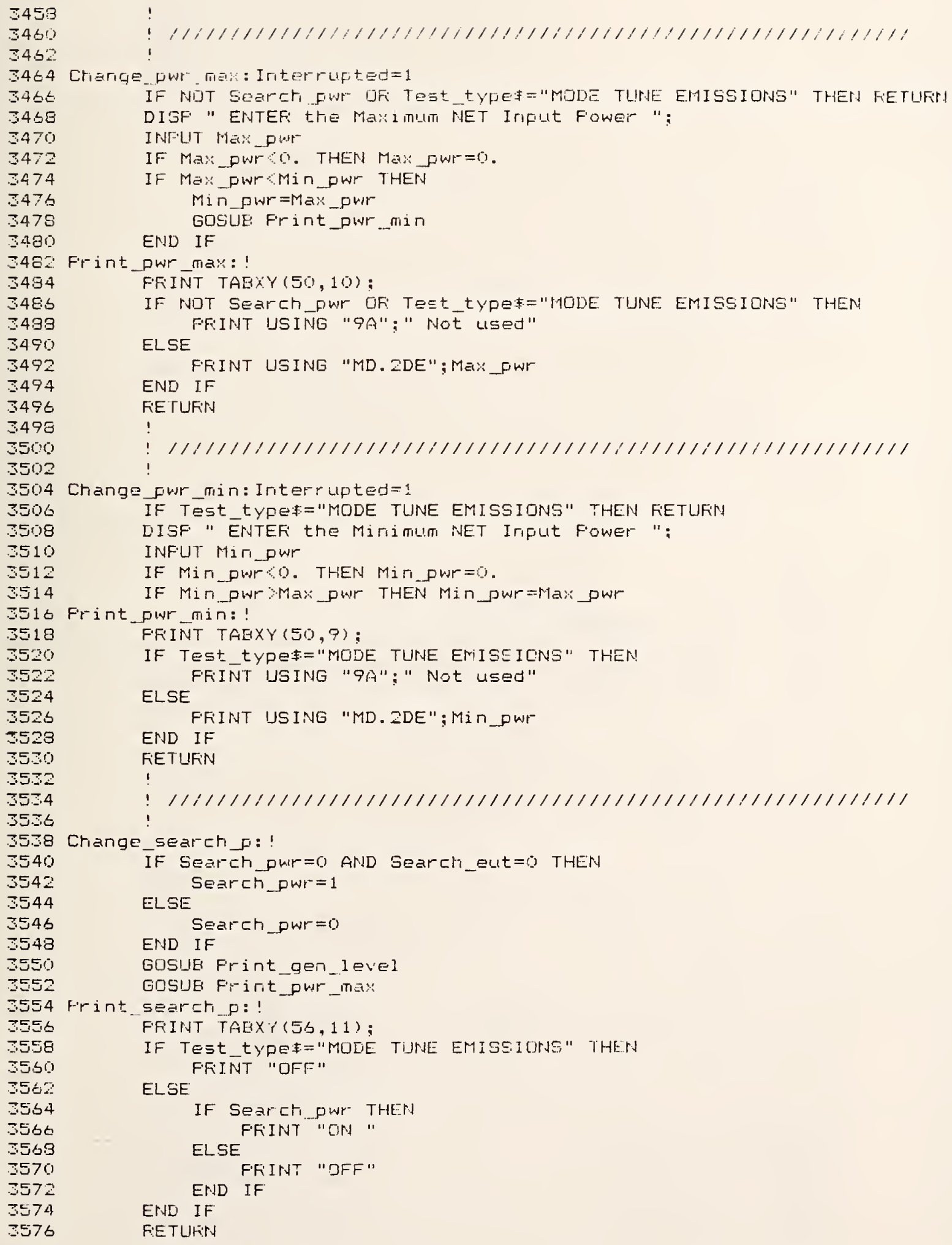




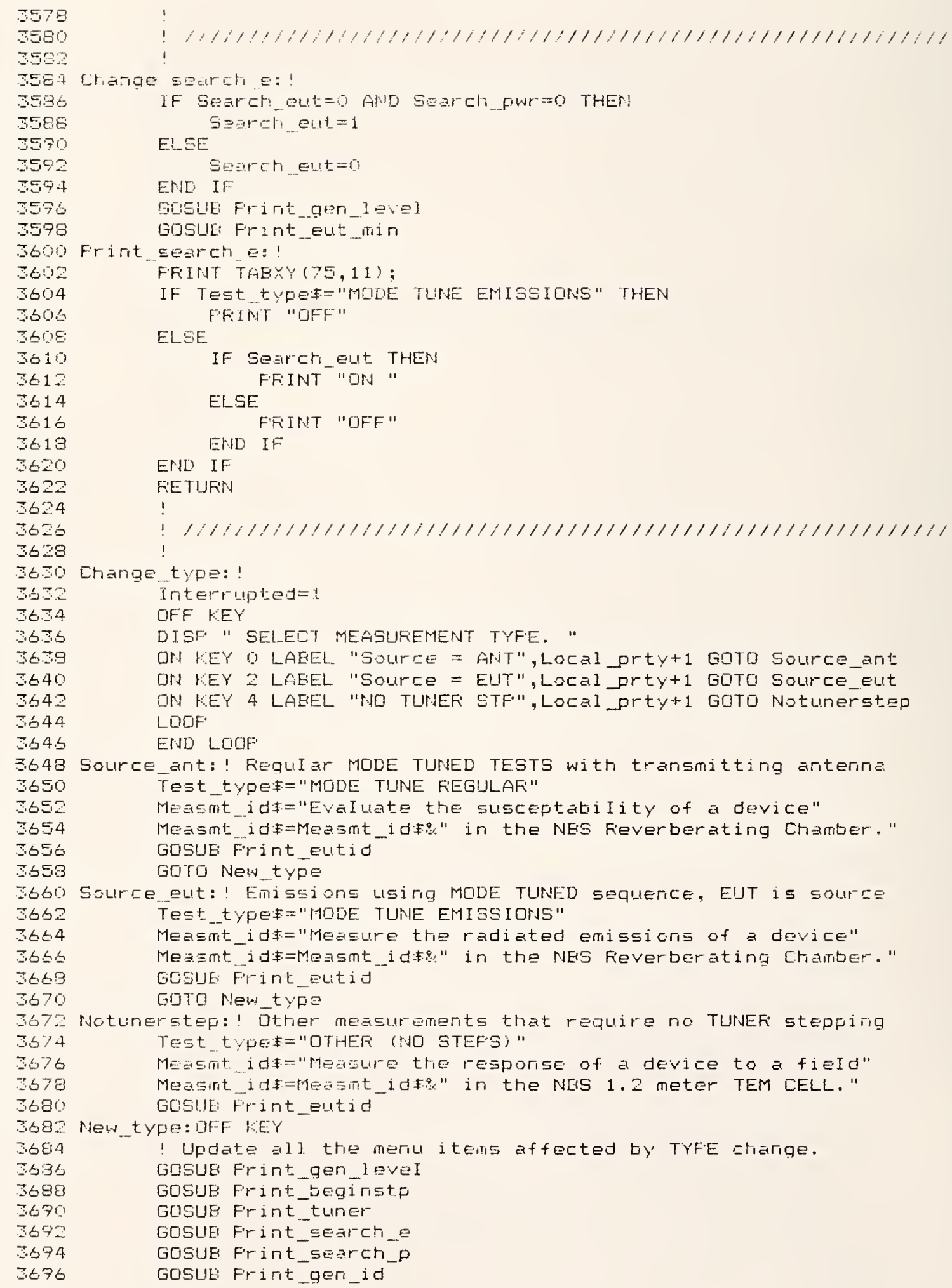




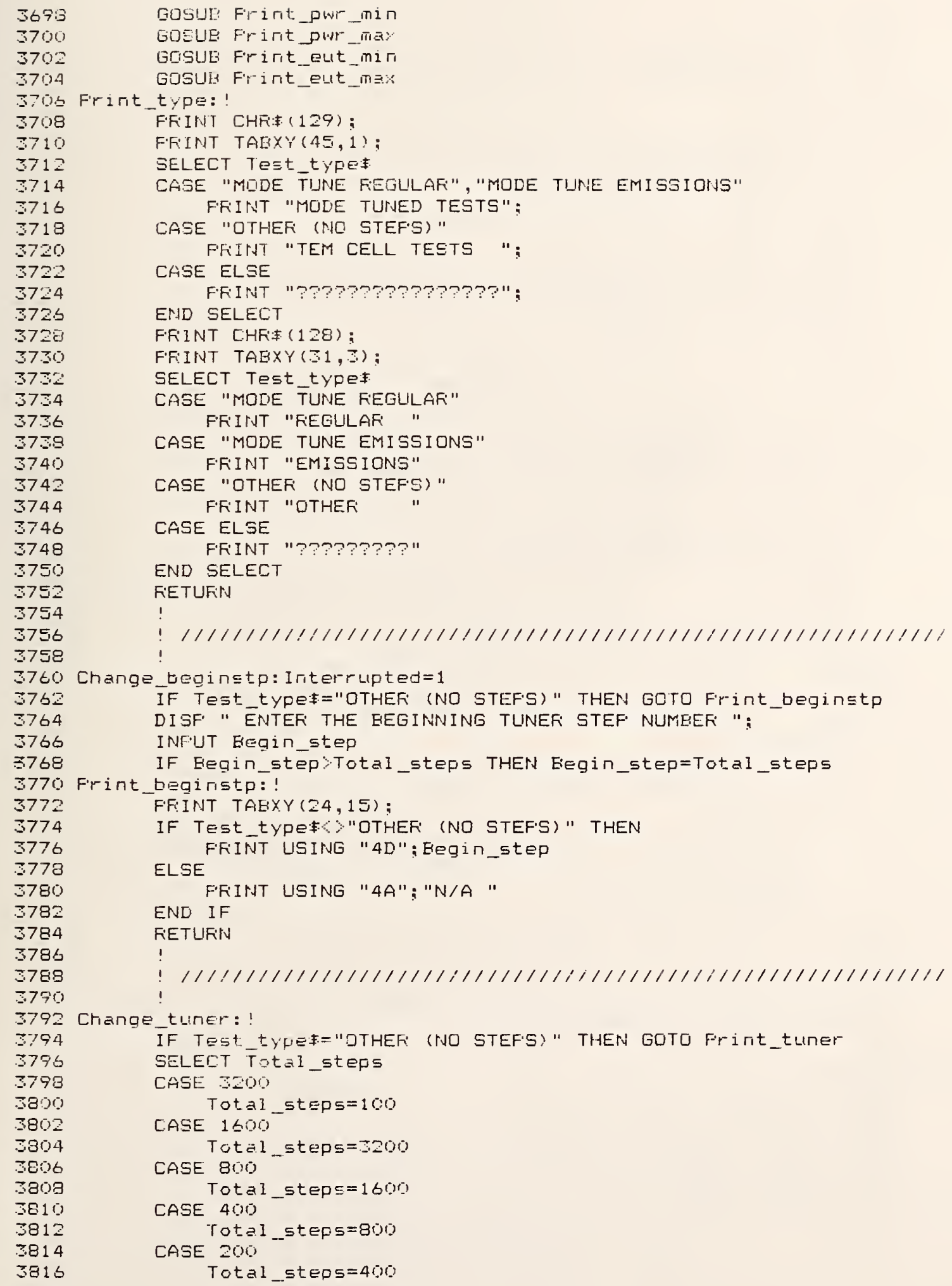




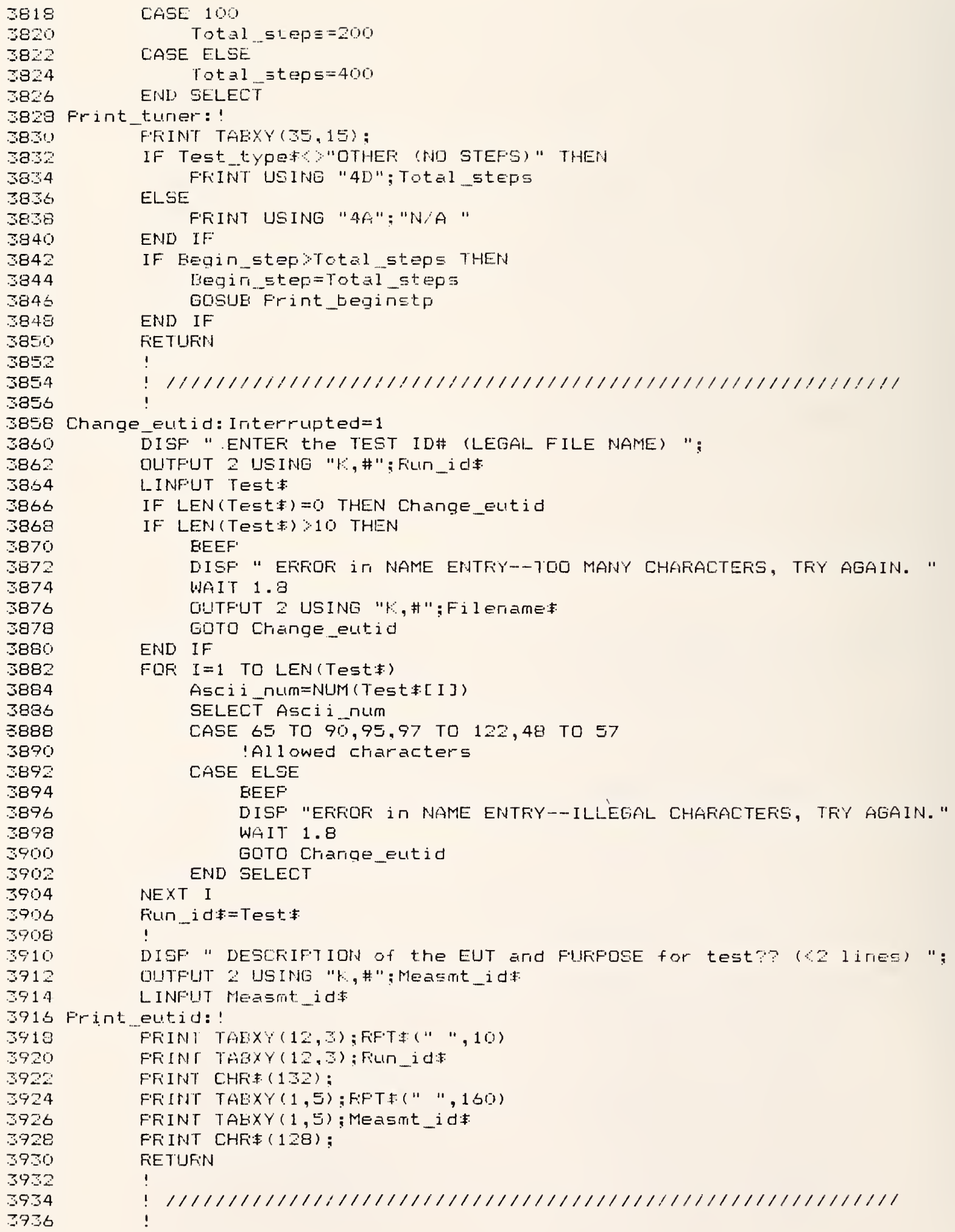




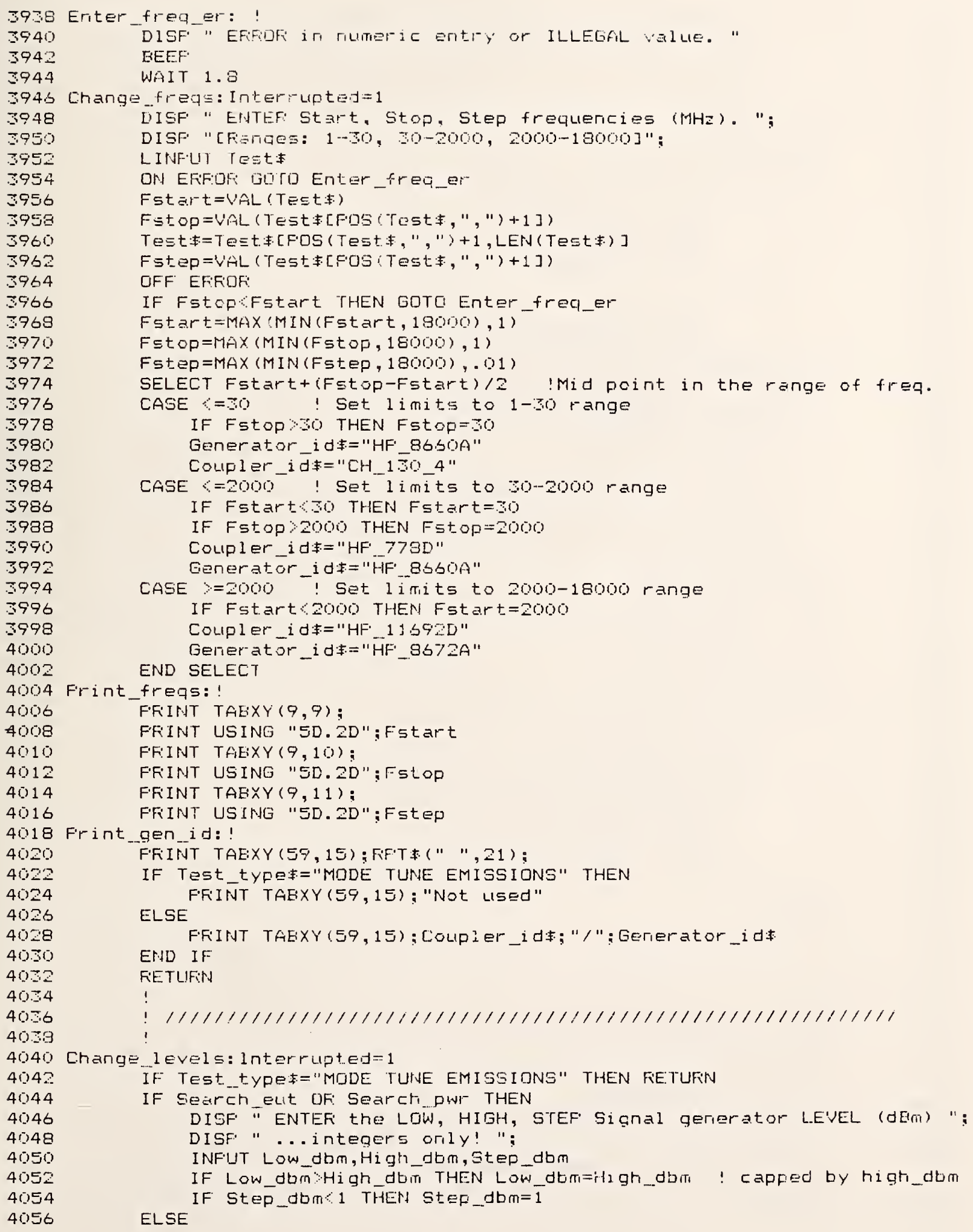




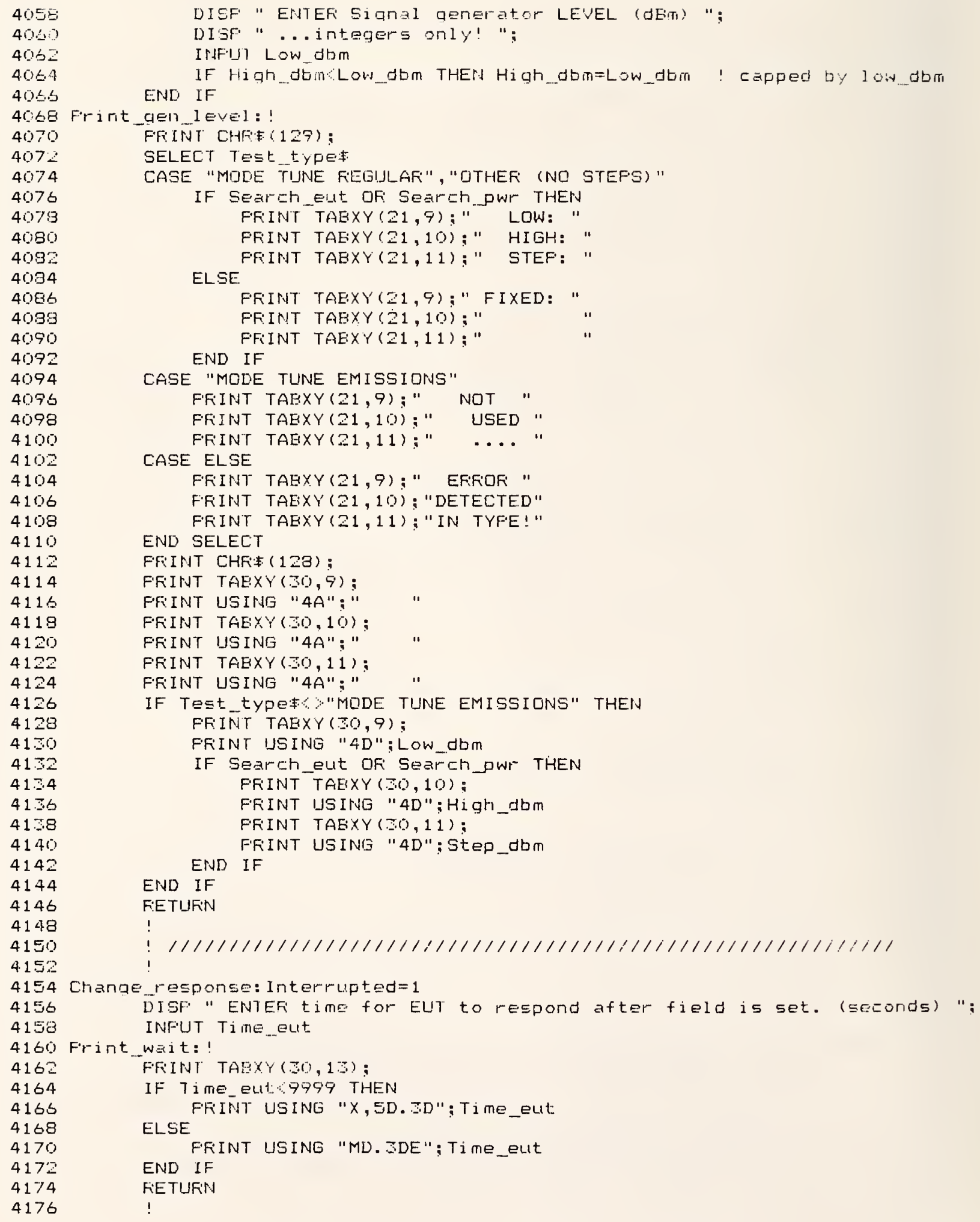




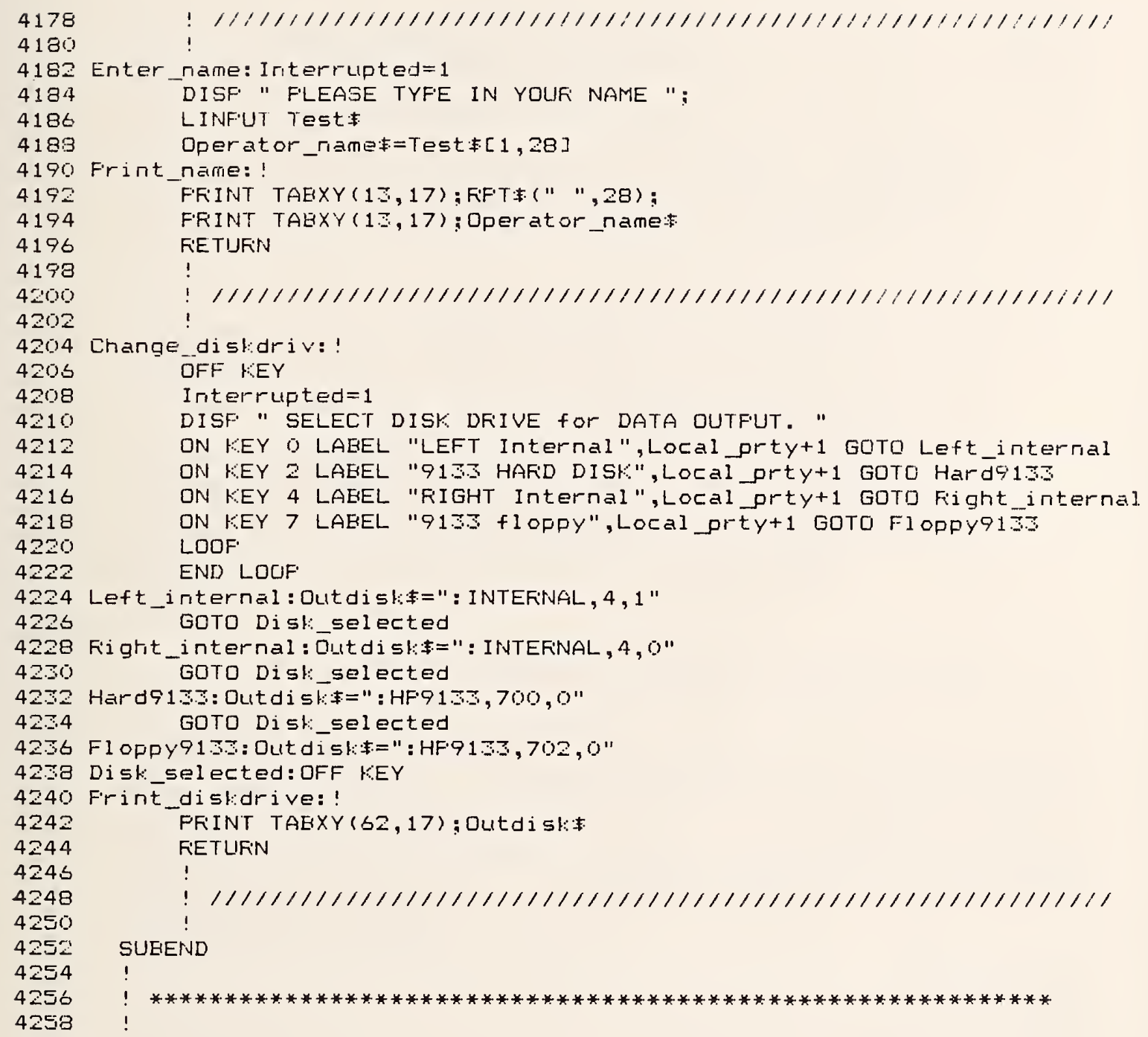




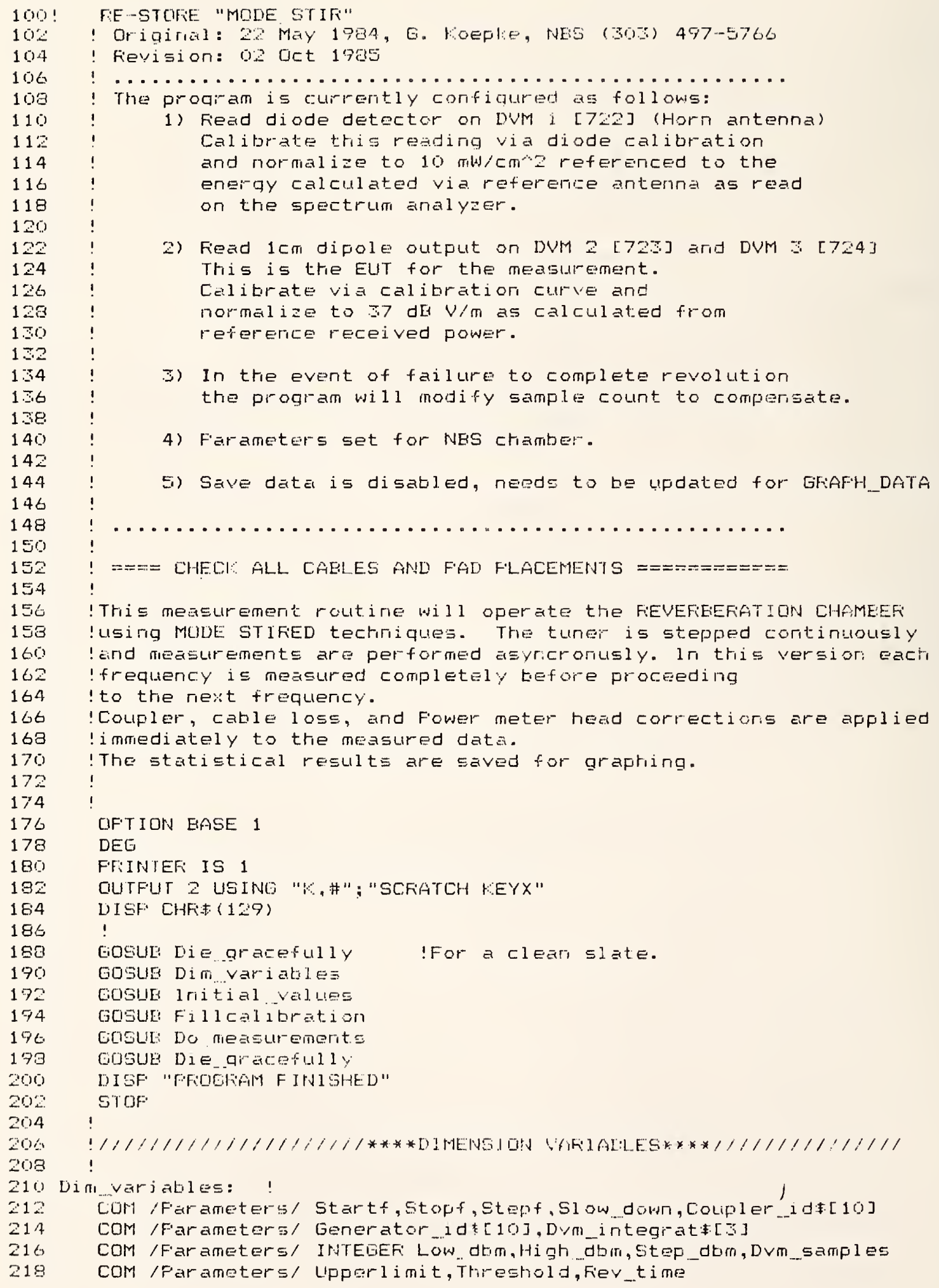

3) In the event of failure to complete revolution the program will modify sample count to comperisate.

4) Farameters set for NES chamber-

5) Save data is disabled, needs to be lupdated for GFAFH_DATA 
COH Motor menu/ Motion_typet[10], INTEGEF IOg Irait

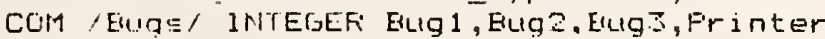

CUM /lnterrupts/ INTEGEFi Intr_prty

Com. Files: Sourcedisl: [20], outdiskt[20],Fil enamets[30]

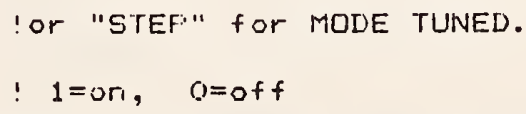

!Go and set up the measurement parameters.

ISet up all power meters

!For a clean slate. 


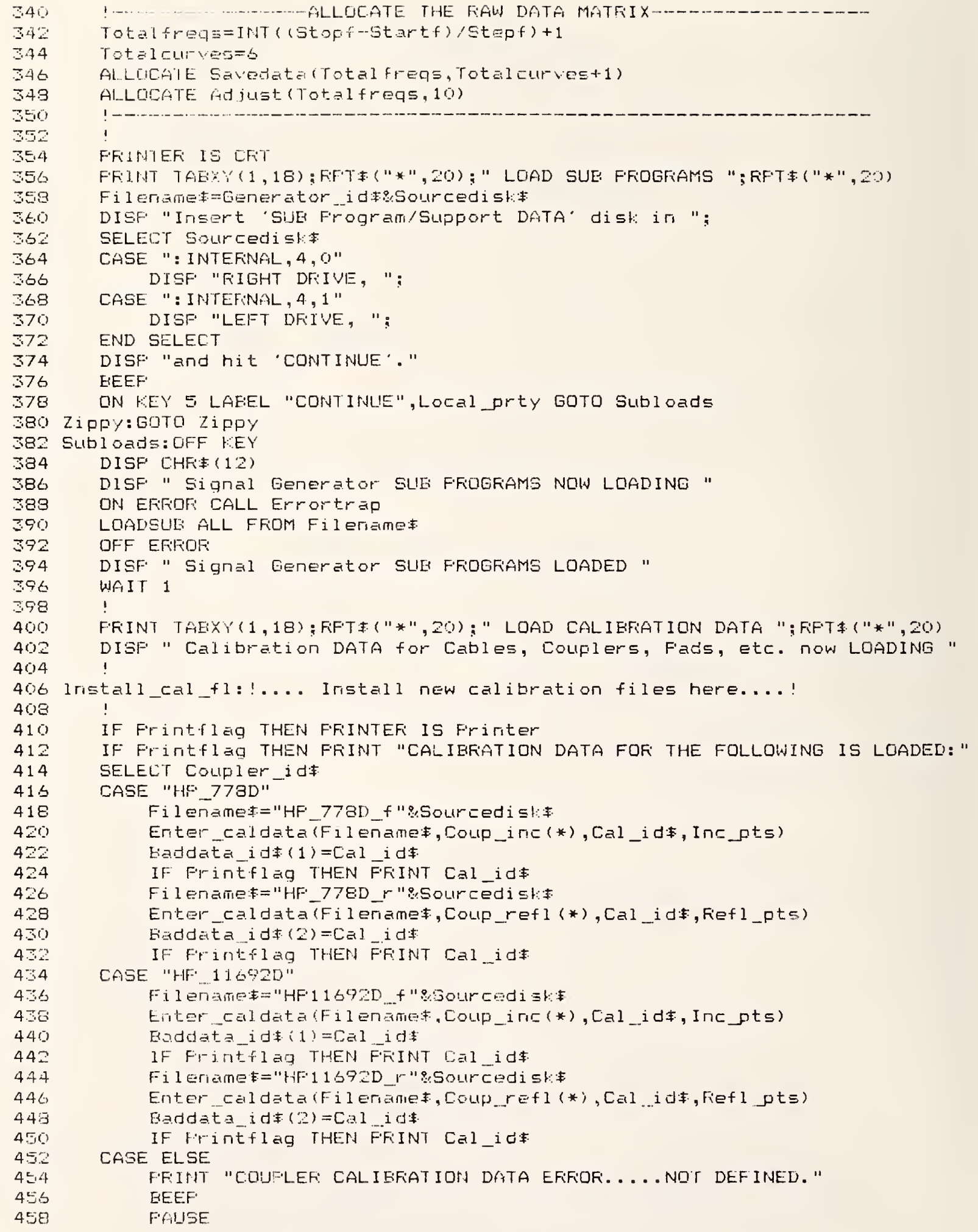




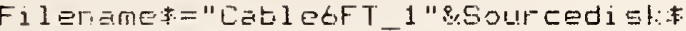

Enter caldata (Fil enamet, Cableba(*), Cal_idt, Cbapts)

Eaddate $i d \neq(3)=[a l$ idd

IF Frintflag THEN FFINT Cal_idt 1

Fi lenaine:="Cable6FT 6"8Sourcedist

Enter caldata (Fil ensimet, Catlesb (*), Cal idt, Cob pts)

Fiaddatz_id韦(4)=Cal_id

If Frintiflag T'HEN Firint Cal idt

!

Fi lenamet:= "Cable4FT._.2"

Enter caldata (F1 lename: Cable4(*), Cal_idt,C4_pts)

Baddata_id韦 $(5)=C a l$ id

If Frintflag THEN FFINT Cal_ido

!

Fi 1 enamet:= "Cable10F_5" 8 Sourcedist:

Enter.caldata (Filenamet,Cable10ft(*),Cal_id*,c10_pts)

Eaddata id $(6)=$ Cal id

IF Frintflag THEN FRIIN Cal_id:

!

Fi 1 ename: = FAD_56770"isourcedist:

Enter_caldata (Fi l enamef, Fad_s6770(*),Cal_idt,Fad_pts)

Baddata_id韦 $(7)=\mathrm{Cal}$ id

IF Firintflag THEN FFIINT Cal_id: !

Fil enamet:- "FAD $55.50 "$ \%

Enter caldata (Filenamet,Fad_f $550($ ) (*) , Cal_id*,Fadf fots)

Baddsta id $(8)=$ Cal id:

IF Frintflag THEN FFINT Cal_id !

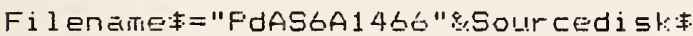

Enter_caldata(Fil enainet,Fad_asba(*), Cal_idt,Fada_pts)

Baddata idf $(9)=$ Cal idt

IF Frintflag THEN FFIINT Cal_idt

Fil lename: ="FdASóA1466"

Fi leriane $=F$ i l ename:o Sourcedist:

Enter_caldata(Filename: Nswc(*), Cal_idit,Nswc.pts)

Eaddata_idt $(10)=$ Cal id

IF Friritflag THEN FFiINT Cal idi...

DISF" " Calibration DATA LDADED "

WAIT 1

FFIINTEER IS CFIT

CALL WIPE_clean

1

!

DISF "INSEFT OUTFUT DATA DISK IN ";

SELECT DUtdISY:\$

CASE "I INTEFINAL, $4,0 "$

DISF "FIIGHT DFIVE, ":

CASE ": INTEFINAL, 4, 1 "

DISF "LEFT DFIVE, ";

END SELECT

DISF "and hit 'CONTINUE'."

BEEF. 
UH lEY 5 LAEEL "COHT INUE", LOE al prty GOTO DatesEver

592 ZiFFity: G0I0 Zippity

584 DEtaEaVEl: UIFF KEY

586 DISF CHFIt (12)

$588 \quad !$

$590 \quad$ FiETUFI

552

594

596

5,98

600

602

604

606

608

610

612

614

616

618

620

622

624

626

628

6.30

6.32

6.4

6.6

68

640

642

644

646

649

650

652

654

656

659

660

662

654

666

668

670

672

674

676

678

680

682

684

$6 E 6$

586

690

692

694

656

698 


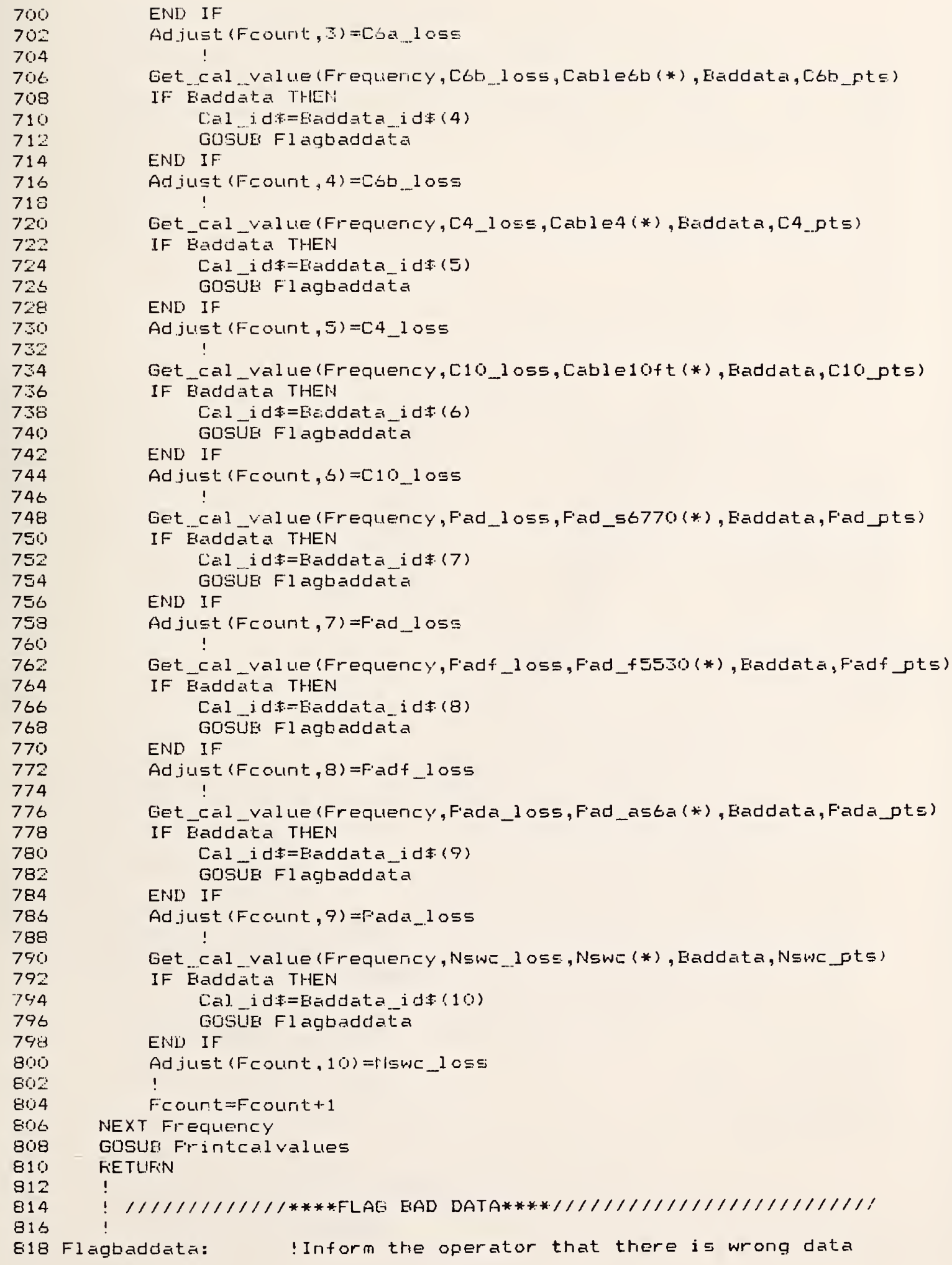

END IF

Ad just (Fcount, 3 ) = Cod 1055

$$
\text { ! }
$$

Eet_cal ...value (Frequericy, Cbb_loss, Cabletb (*), Eiaddata, Cob_fte)

IF Eaddata THEN

Cial i. d

END IF GUSUE Flagbaddata

Ad_iust (Fcount, 4 ) = C.St _.. 1055 !

Get_cal_val ue (Frequency,C4_loss,Cable4(*), Eaddata, C4_pts)

IF Baddata THEN Cal_idt=Eaddata_idt(5)

END IF GoSuk Flagbaddata

Ad just (Fcount, 5 ) $=$ C4_1055

Get_cal_value(Frequency,C10_loss, Cable10ft(*), Eaddata,C10_pts)

IF Eaddita THEN Cal_idt=Eiaddata_idt(6) GOSUE Flagbaddata

Ad just (Fcount, $S)=$ C10_1 oss !

Get_cal_value (Frequency,Fad_105s,Fad_56770(*), Eaddata,Fad_pts)

IF Exddata THEN Cal_idf=Baddata_id (7)

END IF GusUE Flagbaddata

Adjust (Fcount, 7) =Fad_l 05 S !

Get_cal_val ue (Frequency,Fadf_loss,Fad_f5530(*), Eaddata,Fadf_pts)

IF $\bar{E} \equiv d d \overline{a t a}$ THEN Cal j d t:-Faddata_idt. (8)

END IF GUSUE Flagbaddata

Ad just (Fcount, 8) $=F$ 'adf_1055

Get_cal_value (Frequency, Fada_loss, Fad_asta(*), Baddata, Fada_pts)

IF Eaddata THEN Cal__ id:=Eaddata_id (9)

END IF GOSÜE Flagbaddata

Ad just (Fcount, 9 ) =Fada_ 1055 !

Get cal value (Frequericy, Nswe l oss, Nswe (*), Eaddata, Nswe_pts)

IF Eaddata THEN Cal. idt=Eaddata_idt(10)

ENVi) IF GOSLE Flagbaddata

Ad just (Fcount, 10 ) =riswc_l $10 s$ s ! Frcount $=F c$ count +1

NEXT FrequLICICY

gusue Frintcalvalues

FIETLIFIN

!

$1 / 1 / 1 / 1 / 1 / 1 / 1 * * * *$ FLAG EAD DATA*****1/1/1/1/1/1/1/1/1/1/1/1/11

Flagbaddata: $\quad$ Inform the operator that there is wrong data 


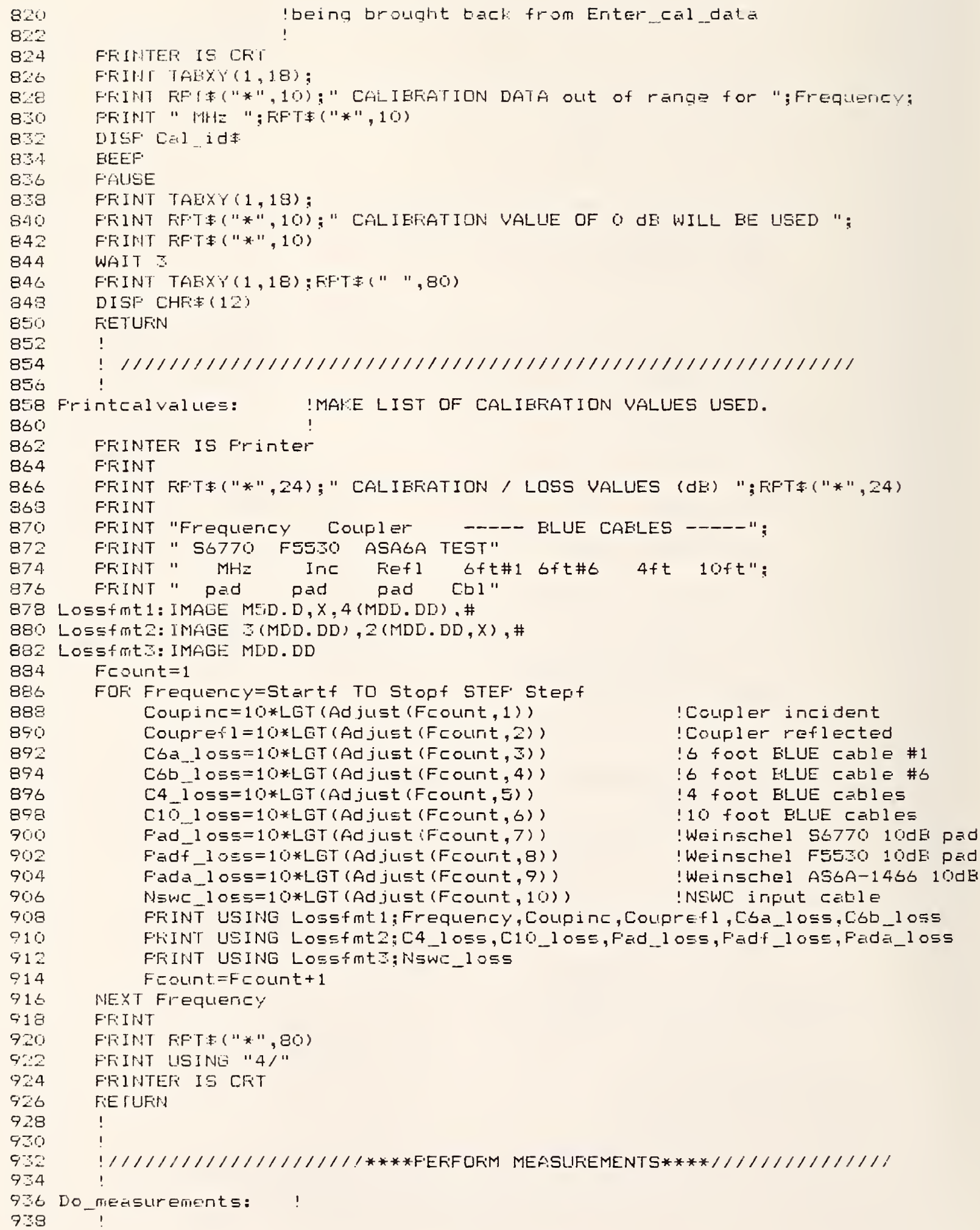




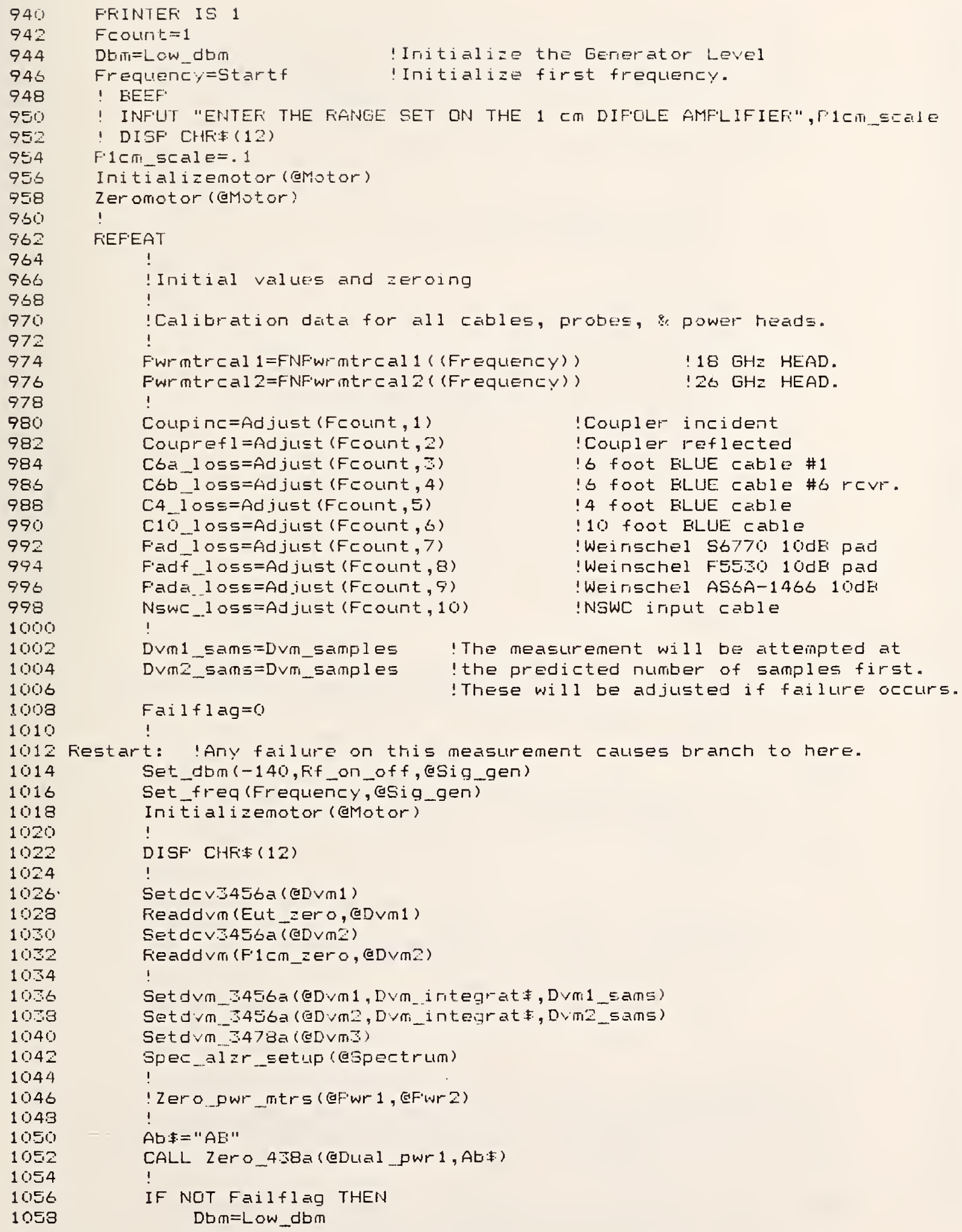




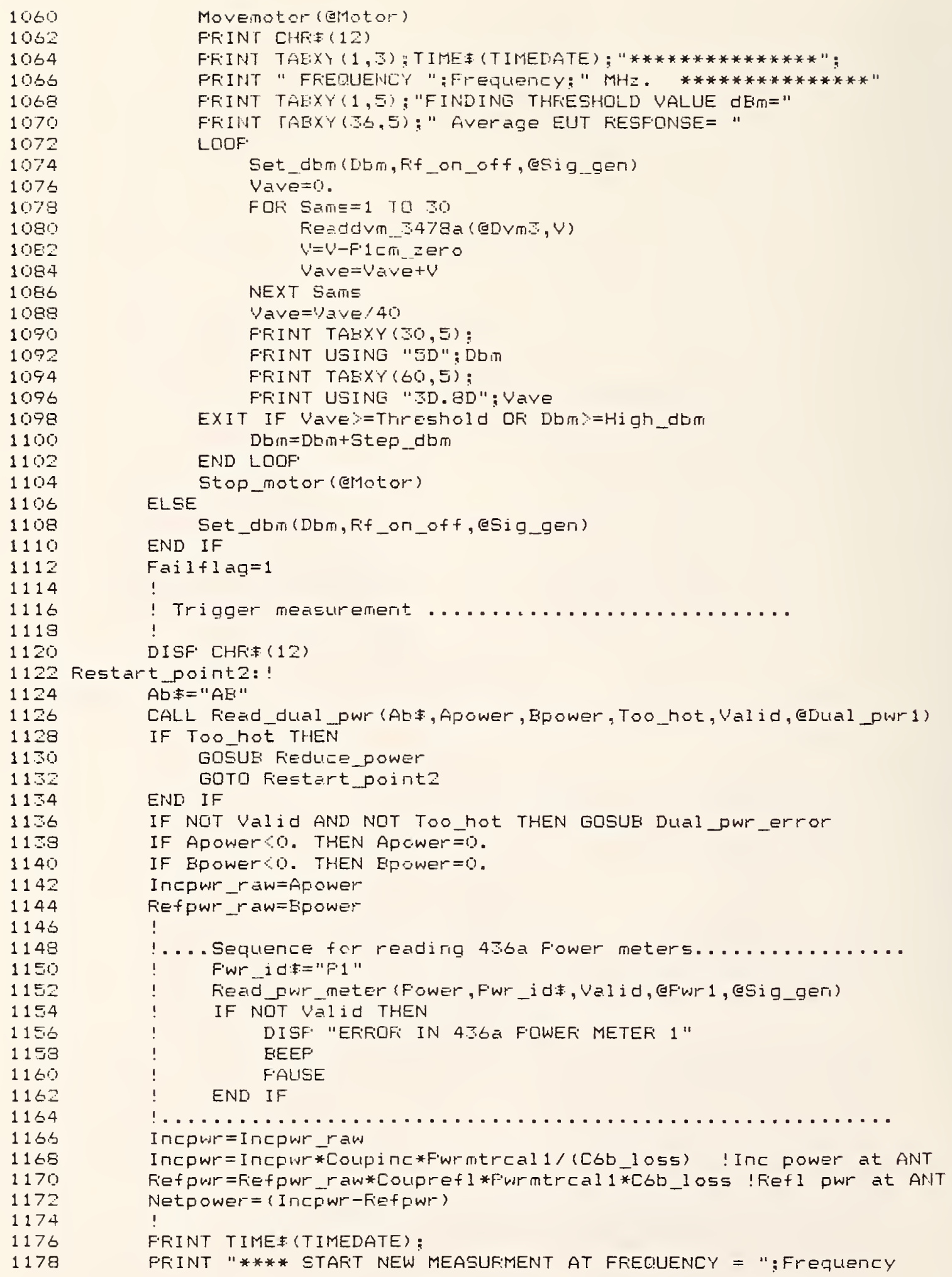

Movenotor (emotor)

F.FINT CHF: (12)

FFIINT TAEXX $(1,3)$ "TIME

FFirdT "FFEQUEVIUY ";Frequency:" MHz. *****************"

F.FINT TAEXY $(1,5)$; "FINDINE THFESHOLD VALUE $d B m="$

FFIUT TABXY $(5,5)$; " fiverage EUT FESFONSE= " LOOF.

END IF

Set_dum(Ltm, fif_on_off, tesig_gen)

Vave=0.

FOF $S a \pi i \subseteq=1$ TO 30

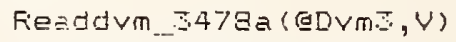

$V^{\prime}=V-F \cdot 1 c \pi$ zero

NEXT SaIIS

Vave=vave+v

Vave= Yave/ 40

FIFINT TABXY $(30,5)$ :

FFIINT USING "SD": DOM

F'FIINT TAEXY $(60,5)$;

FIIINT USING "BD. BD": Vave

EXIT IF Vave $>=T h r e s h o l d$ OF Dbm $=$ High_dum

END LQDF Dbin=Dbin+Step_.dbm

Fai $1+1$ ag $=1$

!

! Trigger measurement .....................

DISF CHF: (12)

Fiestart_point2: !

$A B:=" A B "$

CALL Fiead_dual_pwr (Ab:, Apower, Epower, Too_hot, Valid, GDual_pwr 1)

IF ToO_hot THEN

GOSUE FiEdLIEE_pOWEr

END IF

GOTO Fiestart_point?

IF NCIT Valid AND NOT TOO_hot THEN GQSUE Dual_pwr_error

IF Apower 6 . THEN Apc.wer=0.

IF Epower<0. THEN EPower $=0$.

InCPWr $r$ awhAfower

FiEfPWr $r$ aw =EPOWEr

!

!... Sequence for readirig 4 Sba Fower meters............. F'wr idt="Fi"

Fiead_fur meter (Fower, Fwr_idt, Valid, GFwr 1, GSig_gen)

IF NDT Valid THEN DISF" "EFR:OFi IN 4.6a FOWEF METEF 1 "

EEEF

END IF F.ALISE

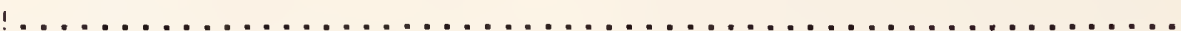

Incpwr=Incpwr_raw

Incpwr=Incfwr *Coupinc*F'wrmtrcal1/(C6b_loss) ! Inc power at ANT Fiefpwr=Fiefpwr_raw*Coupref]*F'wrmtrcal1*C6b_loss ! Fiefl pwr at ANIT Netpower $=$ (Incpwr-Fiefpwr $)$ !

FFIINT TIMEZ. (TIMEDATE):

FFIINT "**** STAFT NEW MEASUFMENT AT FFEQUENCY = ": Frequency 


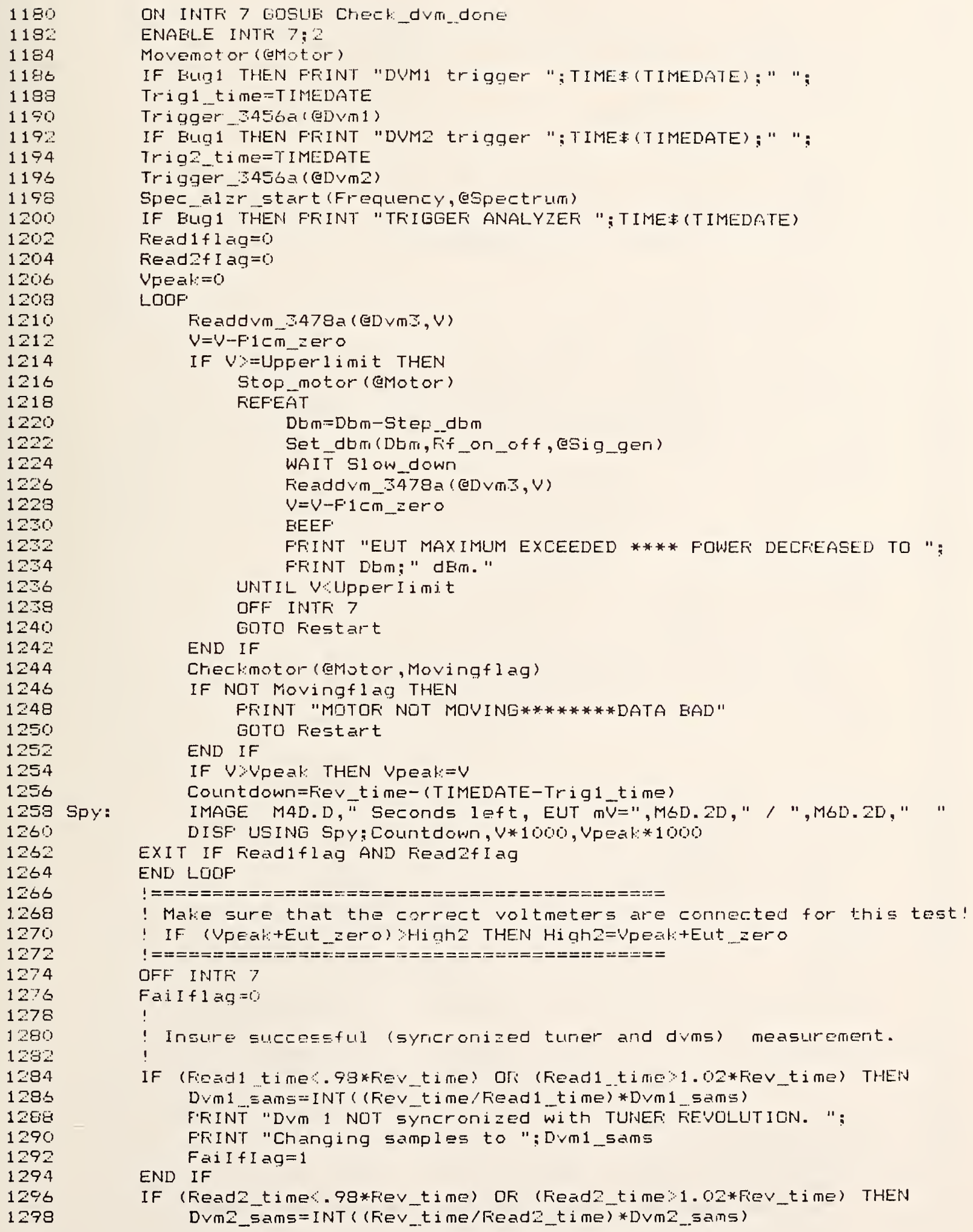

ON INTFi 7 GOSLIE ChECR_dVm dONE

ENAELE INTF $7: 2$

Movemot or (eMotor)

IF ELI 1 THEN FFINT "DVM1 trigger "; TIME (TIMEDÁTE); " "

Trig1 timE=TIMEDATE

Trigger S45óa (ODVM1)

IF EıI 1 THEN FFIINT "DUMZ trigger ": TIME (TIMEDATE):" ";

Trig 2 time=TIMEDATE

Trigger _45ó (@DVma)

Spec alzr start (Frequency, eSpectrum)

IF BUIG 1 THEN FFINT" "TFIGGEF ANALYZEF ": TIME: (TIMEDATE)

Fie ad $1 \mathrm{f} I \mathrm{a} g=0$

Fiead $2 f$ I $a q=0$

VFEE

LOOF'

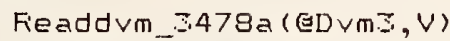

$V=V-F 1 \subset m_{-}=$ero

If $V\rangle=$ Upper 1 imit THEN

Stop_motor (eMotor)

FEFEATT

Dum $=$ Dbm-Step_dbm

Set_dbmi(Dtm,Fif_on_off, ESig_gen)

WAIT Slow_down

Fie addvm_ 3478 (

$V=V-F 1 \subset m_{-}$_ero

EEEF

FIFINT "EUT MAXIMUM EXCEEDED **** FOWEFi DECFEEASED TO ":

FFINT DEm:" dEm."

UNTIL VupperIimjt

OFF INTF 7

END IF

GOTO Fiesta!t

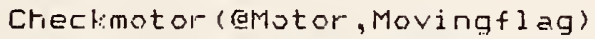

IF NOT Movingflag THEN

FFIINT "MOTOF' NUT MOVING********DATA EAD"

GOTO Fiestart

END IF

IF VVipeal: THEN Vpeat: $=V$

Countdown=Fiev_time-(TIMEDATE-Trig1_time)

IMAGE MAD.D, "Seconds 1 eft, EUT $m \bar{V}="$, MED. $2 \mathrm{D}, "$, ", MGD. $2 D, "$ " DISF USING SpY:Countdown, V*1000, VPEat: 1000

EXIT IF Fieadiflag AND Fieadzf I ag

END LUUF.

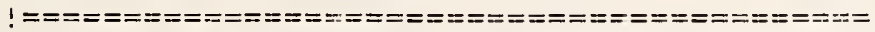

! Mare sure that the correct voltmeters are connected for this test!

! IF (Vpeatiteut_zero) \%Highz THEN Highz=Vpeati+Eut_zero

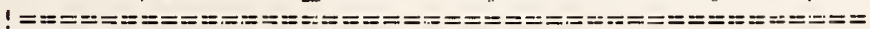

OFF INTF 7

Fai I $f$ a

$!$

! Insure succeseful (syricronized tuner and dvms) measurement.

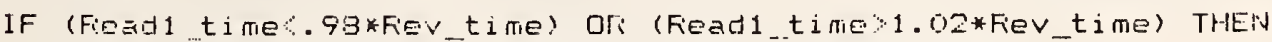

Dvm 1 _...ams=INT ( Fiev_time/Fiead 1 _. $t$ ime) *DVm1_. 5 ams)

FFINT "DVm 1 NOT syncronized with TUNEF: FEVUOLLITION. ":

FFint "Changing samples to "; Drm1_sams

END IF

Fai I $f$ I $g=1$

IF (Fiead2_times.98*Fiev_time) OF (Fiead2_time 1.02*Fiev_time) THEN DVm2_sams =INT ( Fiev_time/Fiead2_time) $*$ DVm2_.sams) 
1500

1.02

1304

$1: 06$

1.08

1510

$131=$

$1: 14$

$1: 16$

1.19

1.20

1.322

1.324

1.326

1.328

1.30

13.32

1.3 .5

1356

1.38

1.50

1.72

$1: 54$

1345

1.548

1.50

1352

1.54

1 156

1.358

1.60

1.62

1.64

1.565

1.368

1.370

1372

1.74

1.576

1.78

1.580

1.392

1.584

1.53 .5

1.588

1390

1.372

1594

1.96

1.99

1400

1402

1404

1406

1408

1410

1412

1414

1416

1418

END IF

FFilNT "Lvm 2 NOT SYRErorized with TUNEFi FEVULUTION. ";

Fifint "Changing samples to "; Dvm? sams

$F$ Ei $1+1$ a $q=1$

IF Fil 1 flag THEN

Stop_motor (eMotor)

END IF Golo Festart

Spec_alar_read (Fower, Frequency, eSpectr-um)

FFINT "SFECTFIUM ANALYZEF: READS "iFower: AT FREQUENCY "ifrequency Stop_motor (erlotor)

Set_dbm(-140,fif_on__off, ๔Sig_gen $)$

!

GUSUF Frocess data

GOSUG Frint_alldata

!

Frequency=Frequency+Stepf

Fcouint $=F c$ ount +1

UNTIL Frequency $>$ stopf

!

Set_dbm (-140,0, (eSig_gen)

! GŌSUE Save_data

FiETUFil

I

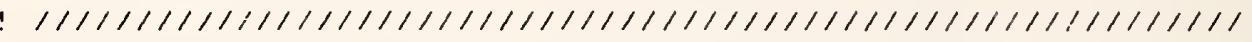

Fieduce power: !

$D b m=\overline{D b m}-1$

Set dum(Dbm, 1, dSig gen)

IF Firintflag THEN

FFIINTEF IS F'rinter

FFIINT TIMEF(TIMEDATE); ": Freq="; Frequency;

END IF

F'FINT FiF'T:("*", IO);" NEW GENEFIATDR LEVEL =";Dbm

FEETUFN

!

: 1111111111111111111111111111111111111111111111111111111

!

Dual_owr_error: !

Set_db $\bar{m}(-140,0$, «Sig_gen)

IF Firiritflag THEN

FFINTEF IS Firinter

FFIINT TIME (TIMEDATE): ": Freq="; FrequENCY;

FFINT FFFT ("*",10);" EFFFFi IN DUAL FOUEF METEF !!!"

END IF

STOF ! Frogram dies here!

FET TUFN

!

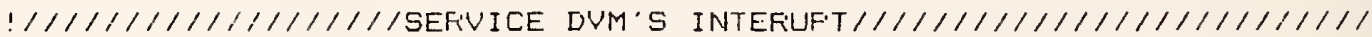
!

Chect_dvi_done:

? Fiespond to an INTEFiUF'T on the HFIE generated ty orie of ! two HF.jusa DUMs. Meaning that the data is ready.

Ser pol1=SF.OLL (@DVM1)

ENAELE INTFi 7

IF Ser poll=6S THEN

Fiead 1_time=TIMEDATE-Trig1_t i ne

IF Bug̣ 1 THEN FRINT "DVM1 total sample time = "; Fiead1_time;" ";

IF EUGI THEN FFINT TIME (TIMEDATE) 


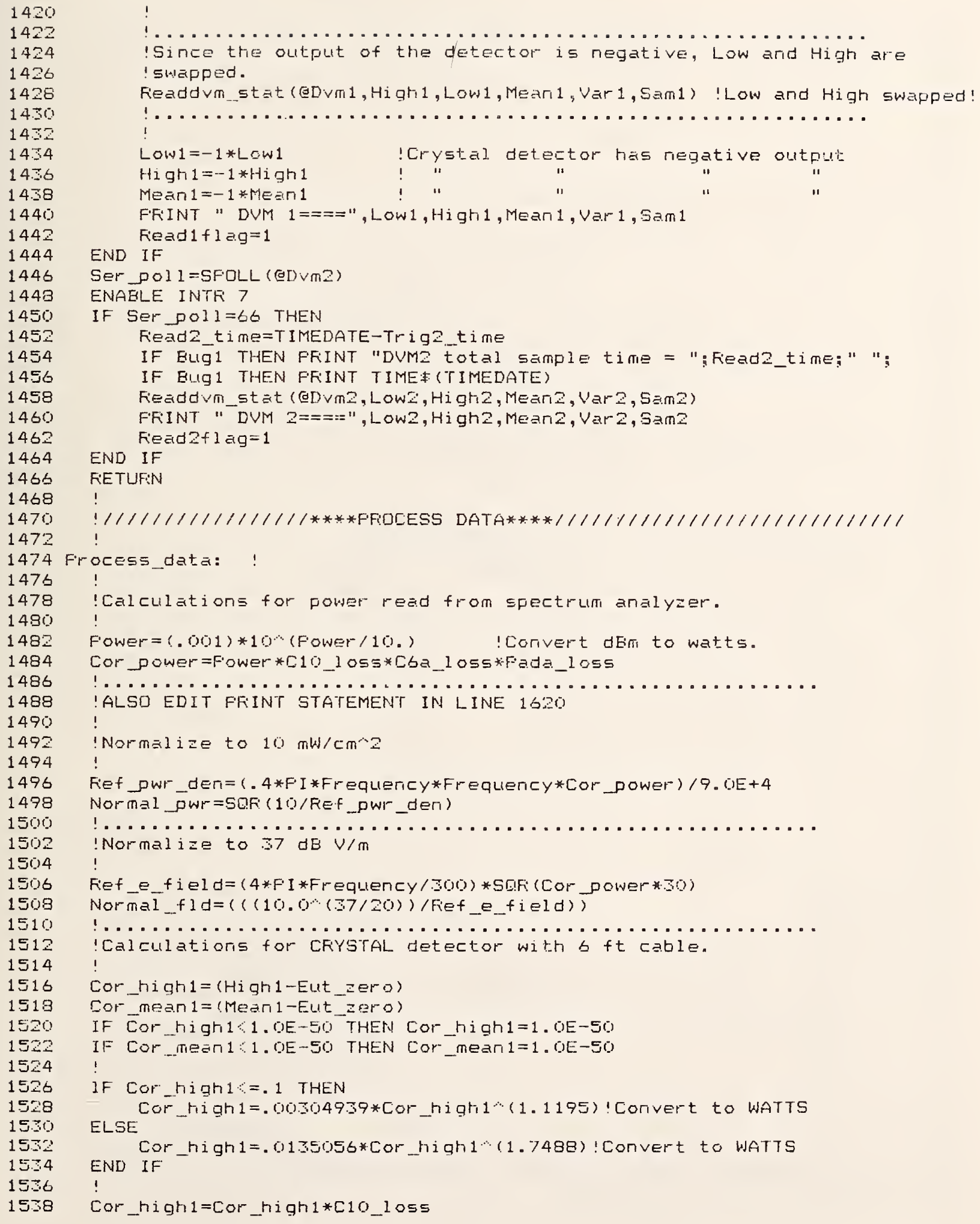




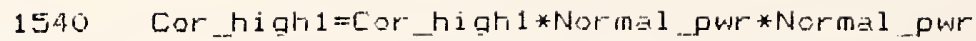




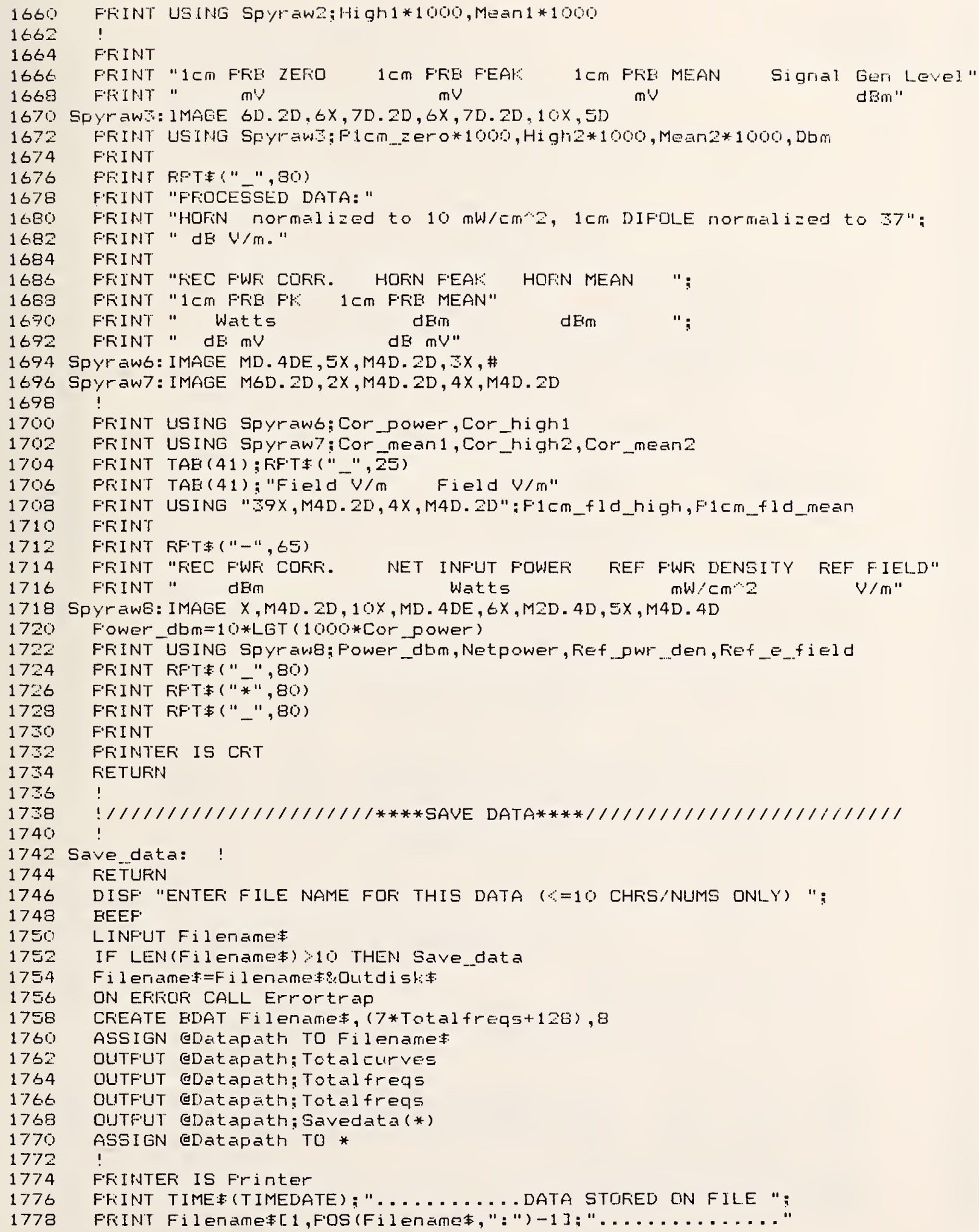




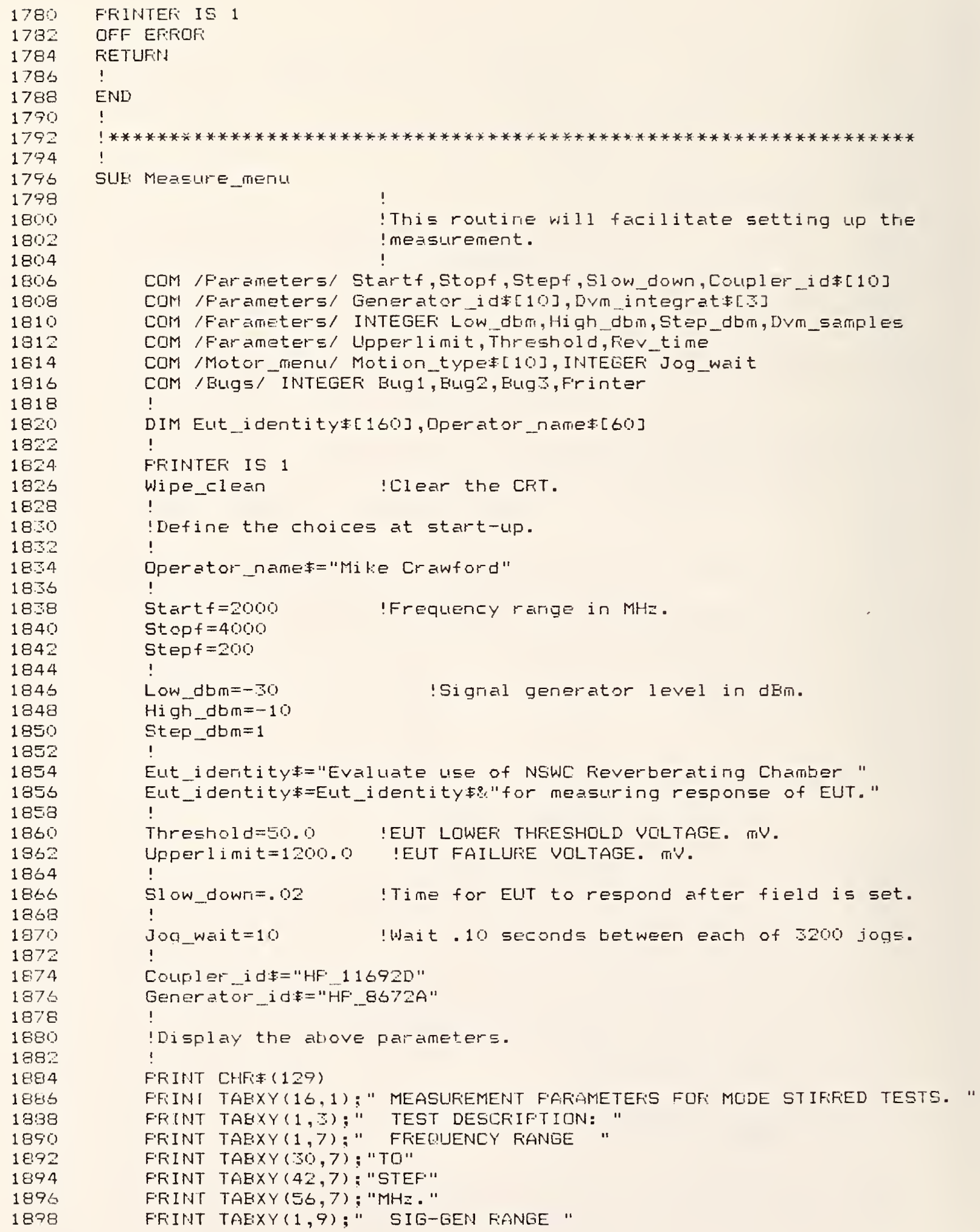
! This routine will facilitate setting up the !messurement. 


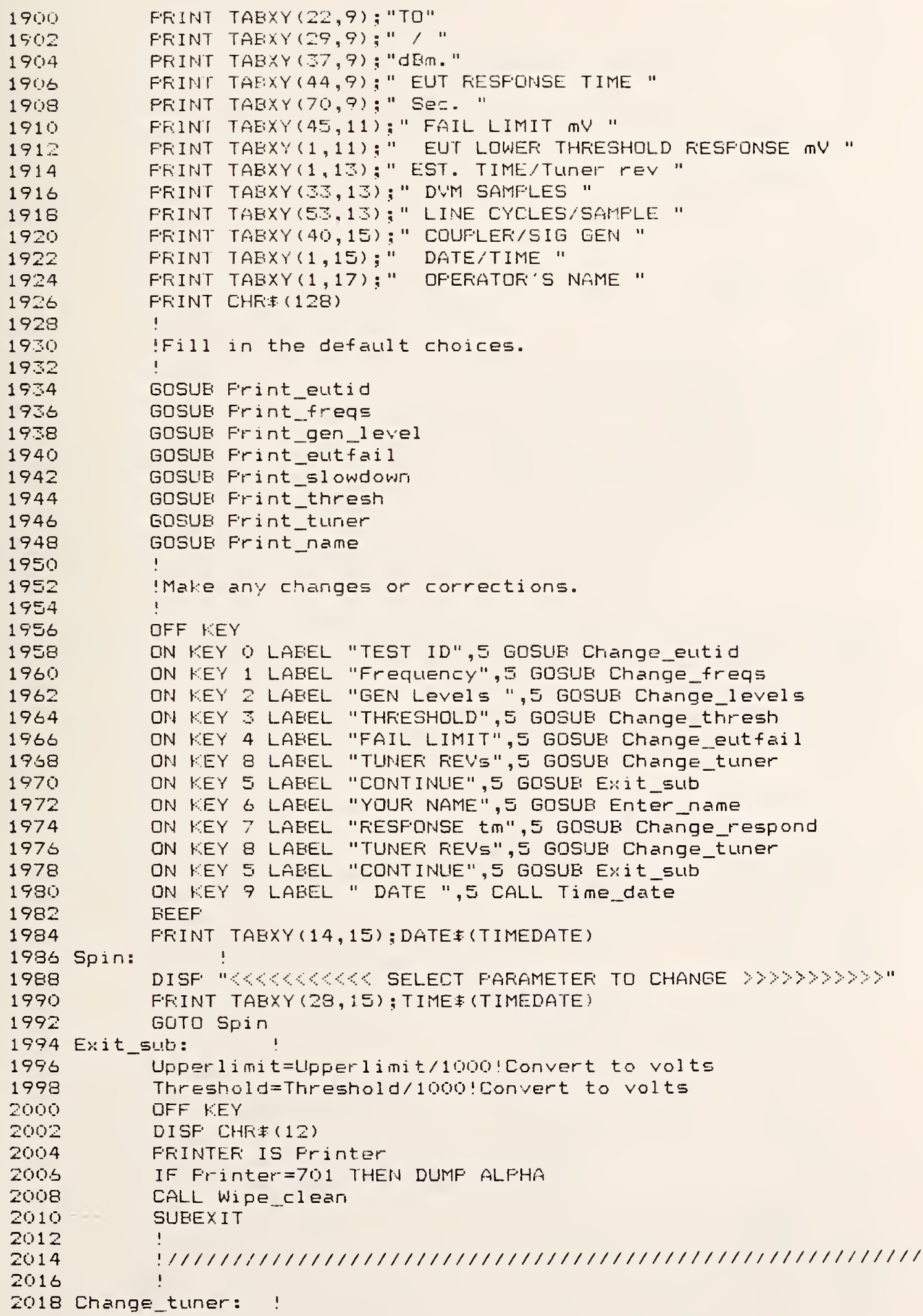




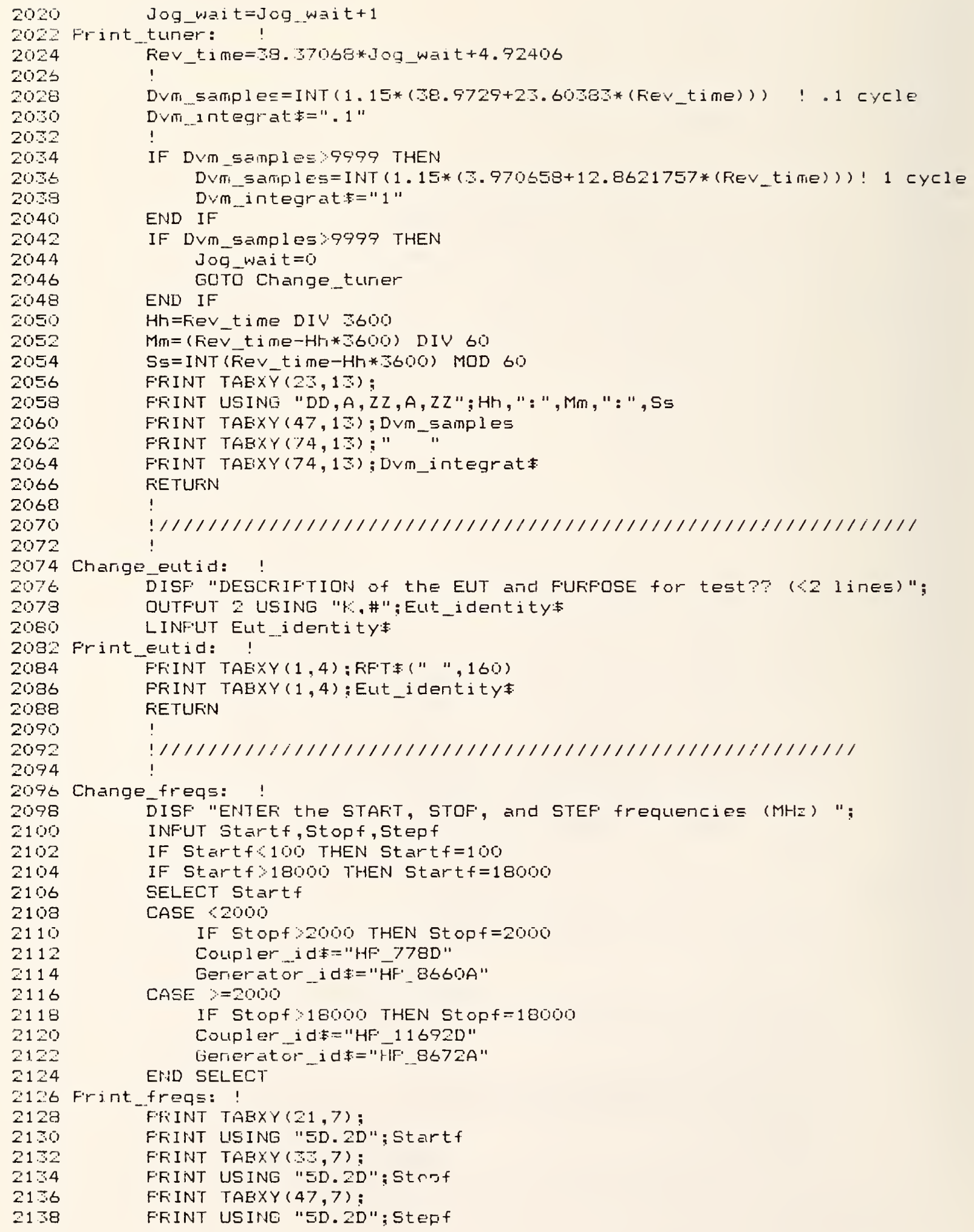




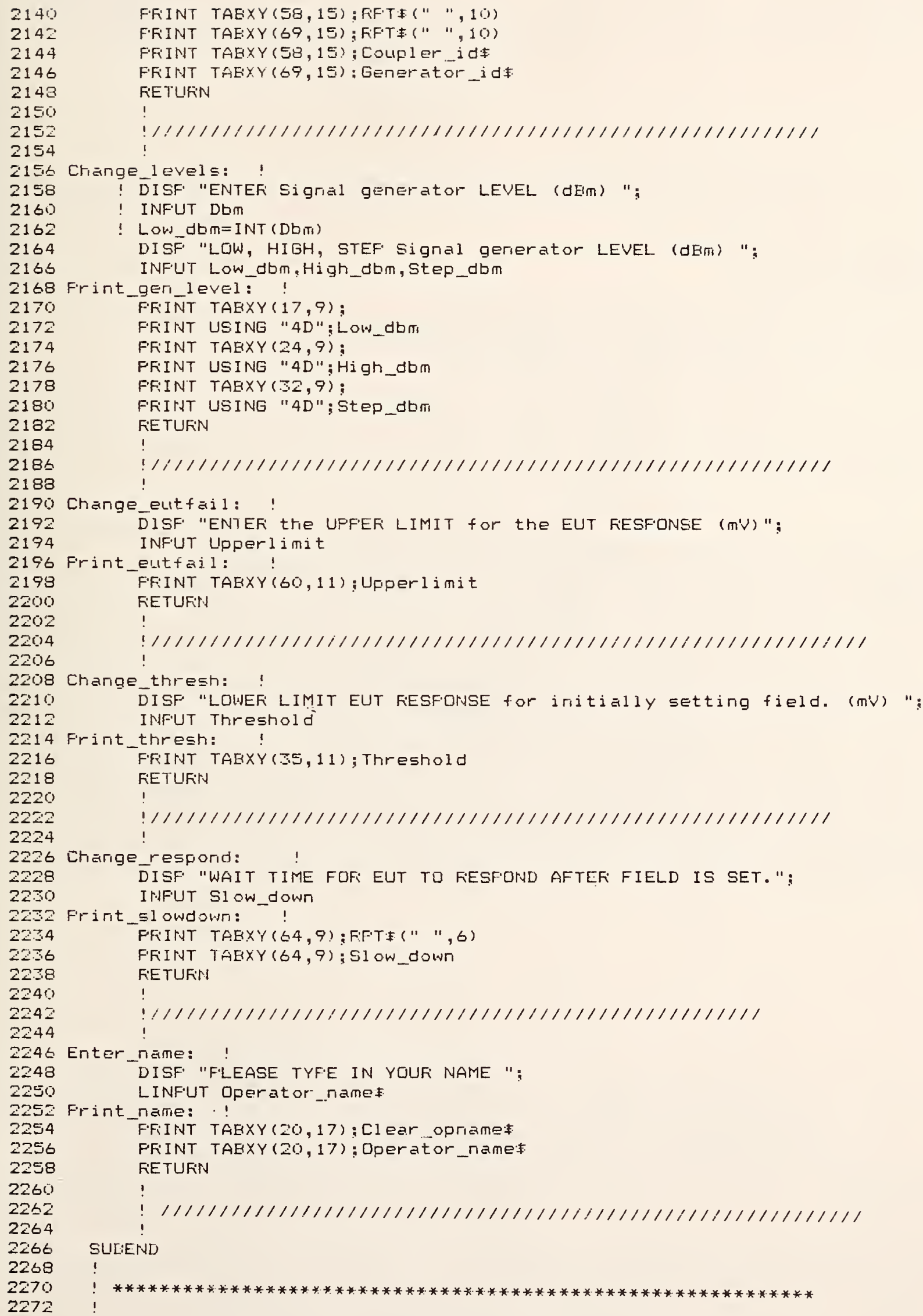


BIBLIOGRAPHIC DATA

SHEET (See instructions)

1. PUBLICATION OR REPORT NO.

NBS/TN-1092
2. Performing Organ. Report No. 3. Publication Date

April 1986

4. TITLE AND SUBTITLE

Design, Evaluation, and Use of a Reverberation Chamber for Performing

Electromagnetic Susceptibility/Vulnerability Measurements

5. $A \cup T H O R(S)$

M. L. Crawford and G. H. Koepke

6. PERFORMING ORGANIZATION (If joint or other than NBS, see instructions)

7. Contract/Grant No.

NATIONAL BUREAU OF STANDARDS

DEPARTMENT OF COMMERCE

WASHINGTON, D.C. 20234

8. Type of Report \& Period Covered

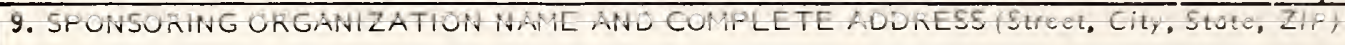

Naval Surface Weapons Center (NSWC), Dahlgren, Virginia

and

Rome Air Development Center (RADC), Griffiss Air Force Base, Rome, New York

10. SUPPLEMENTARY NOTES

Document describes a computer program; SF-185, FIPS Software Summary, is attached.

11. ABSTRACT (A 200-word or less factual summary of most significant information. If document includes a significant bibliography or literature survey. mention it here)

This report presents the results of work at the National Bureau of Standards, Boulder, Colorado, to carefully evaluate, document, develop (when necessary). and describe the methodology for performing radiated susceptibility/vulnerability measurements using a reverberation chamber. The report describes the reverberation chamber theory of operation, construction, evaluation, functional operation, and use for performing immunity measurements. It includes an estimate of measurement uncertainties derived empirically from test results and from comparisons with anechoic chamber measurements. Finally, it discusses the limitations and advantages of the measurement technique to assist potential users in determining the applicability for this technique to their electromagnetic compatibility (EMC) measurement needs.

12. KEY WORDS (Six to twelve entries; alphabetical order; capitalize only proper names; and separate key words by semicolons)

electromagnetic susceptibility/vulnerability; estimated uncertainty; evaluation; measurement procedures; reverberabtion chamber

\section{AVAILABILITY}

K Unlimited

For Official Distribution. Do Not Release to NTIS

X Order From Superintendent of Documents, U.S. Government Printing Office, Washington, D.C. 20402.

Order From National Technical Information Service (NTIS), Springfield, VA. 22I6I 




\section{Periodical}

Journal of Research-The Journal of Research of the National Bureau of Standards reports NBS research and development in those disciplines of the physical and engineering sciences in which the Bureau is active. These include physics, chemistry, engineering, mathematics, and computer sciences. Papers cover a broad range of subjects, with major emphasis on measurement methodology and the basic technology underlying standardization. Also included from time to time are survey articles on topics closely related to the Bureau's technical and scientific programs. 1ssued six times a year.

\section{Nonperiodicals}

Monographs-Major contributions to the technical literature on various subjects related to the Bureau's scientific and technical activities.

Handbooks-Recommended codes of engineering and industrial practice (including safety codes) developed in cooperation with interested industries, professional organizations, and regulatory bodies.

Special Publications-Include proceedings of conferences sponsored by NBS, NBS annual reports, and other special publications appropriate to this grouping such as wall charts, pocket cards, and bibliographies.

Applied Mathematics Series-Mathematical tables, manuals, and studies of special interest to physicists, engineers, chemists, biologists, mathematicians, computer programmers, and others engaged in scientific and technical work.

National Standard Reference Data Series-Provides quantitative data on the physical and chemical properties of materials, compiled from the world's literature and critically evaluated. Developed under a worldwide program coordinated by NBS under the authority of the National Standard Data Act (Public Law 90-396).

NOTE: The Journal of Physical and Chemical Reference Data (JPCRD) is published quarterly for NBS by the American Chemical Society (ACS) and the American Institute of Physics (AIP). Subscriptions, reprints, and supplements are available from ACS, 1155 Sixteenth St., NW, Washington, DC 20056.

Building Science Series-Disseminates technical information developed at the Bureau on building materials, components, systems, and whole structures. The series presents research results, test methods, and performance criteria related to the structural and environmental functions and the durability and safety characteristics of building elements and systems.

Technical Notes-Studies or reports which are complete in themselves but restrictive in their treatment of a subject. Analogous to monographs but not so comprehensive in scope or definitive in treatment of the subject area. Often serve as a vehicle for final reports of work performed at NBS under the sponsorship of other government agencies.

Voluntary Product Standards--Developed under procedures published by the Department of Commerce in Part 10, Title 15, of the Code of Federal Regulations. The standards establish nationally recognized requirements for products, and provide all concerned interests with a basis for common understanding of the characteristics of the products. NBS administers this program as a supplement to the activities of the private sector standardizing organizations.

Consumer Information Series-Practical information, based on NBS research and experience, covering areas of interest to the consumer. Easily understandable language and illustrations provide useful background knowledge for shopping in today's technological marketplace.

Order the above NBS publications from: Superintendent of Documents, Government Printing Office, Washington, DC 20402.

Order the following NBS publications-FIPS and NBSIR's-from the National Technical Information Service, Springfield, VA 22161.

Federal Information Processing Standards Publications (FIPS PUB)-Publications in this series collectively constitute the Federal Information Processing Standards Register. The Register serves as the official source of information in the Federal Government regarding standards issued by NBS pursuant to the Federal Property and Administrative Services Act of 1949 as amended, Public Law 89-306 (79 Stat. 1127), and as implemented by Executive Order 11717 (38 FR 12315, dated May 11, 1973) and Part 6 of Title 15 CFR (Code of Federal Regulations).

NBS Interagency Reports (NBSIR)-A special series of interim or final reports on work performed by NBS for outside sponsors (both government and non-government). In general, initial distribution is handled by the sponsor; public distribution is by the National Technical Information Service, Springfield, VA 22161, in paper copy or microfiche form. 
Gaithersburg, MD 20899

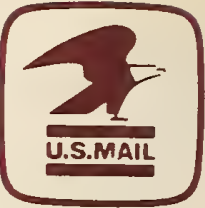

Official Business

Penalty for Private Use $\$ 300$

POSTAGE AND FEES PAID U.S DEPARTMENT OF COMMERCE COM-215

SPECIAL FOURTH-CLASS RATE BOOK 